\title{
Computer-tailored interventions for smoking cessation : targeting smokers with low readiness to quit
}

Citation for published version (APA):

Dijkstra, A. (1998). Computer-tailored interventions for smoking cessation : targeting smokers with low readiness to quit. [Doctoral Thesis, Maastricht University]. Universiteit Maastricht. https://doi.org/10.26481/dis.19980702ad

Document status and date:

Published: 01/01/1998

DOI:

10.26481/dis.19980702ad

Document Version:

Publisher's PDF, also known as Version of record

Please check the document version of this publication:

- A submitted manuscript is the version of the article upon submission and before peer-review. There can be important differences between the submitted version and the official published version of record.

People interested in the research are advised to contact the author for the final version of the publication, or visit the DOI to the publisher's website.

- The final author version and the galley proof are versions of the publication after peer review.

- The final published version features the final layout of the paper including the volume, issue and page numbers.

Link to publication

\footnotetext{
General rights rights.

- You may freely distribute the URL identifying the publication in the public portal. please follow below link for the End User Agreement:

www.umlib.nl/taverne-license

Take down policy

If you believe that this document breaches copyright please contact us at:

repository@maastrichtuniversity.nl

providing details and we will investigate your claim.
}

Copyright and moral rights for the publications made accessible in the public portal are retained by the authors and/or other copyright owners and it is a condition of accessing publications that users recognise and abide by the legal requirements associated with these

- Users may download and print one copy of any publication from the public portal for the purpose of private study or research.

- You may not further distribute the material or use it for any profit-making activity or commercial gain

If the publication is distributed under the terms of Article $25 \mathrm{fa}$ of the Dutch Copyright Act, indicated by the "Taverne" license above, 
Computer-tailored interventions for smoking cessation: Targeting smokers with low readiness to quit 

Computer-tailored interventions for smoking cessation:

Targeting smokers with low readiness to quit

\section{PROEFSCHRIFT}

ter verkrijging van de graad van doctor aan de Universiteit Maastricht, op gezag van de Rector Magnificus,

Prof. Dr. A.C. Nieuwenhuijzen Kruseman, volgens het besluit van het College van Dekanen,

in het openbaar te verdedigen op donderdag 2 Juli 1998 om 14.00 uur

door

Arie Dijkstra 


\section{Promotoren:}

Prof. dr. H. De Vries

Prof. dr. G.J. Kok

\section{Beoordelingscommissie:}

Prof. dr. H. Merckelbach (voorzitter)

Dr. A. Landeweerd

Prof. dr. S. Maes (Rijksuniversiteit Leilden)

Prof. dr. V. Strecher (University of Michigan, United States of America)

Dr. J. Talmon

ISBN: 90-9011516-1

The studies presented in this thesis were conducted at the Institute HEALTH, part of the Netherlands School of Primary Care Research (CaRe), acknowledged in 1995 by the Royal Dutch Academy of Science (KNAW). The studies presented in this thesis were funded by a grant from the Dutch Cancer Society (grant RL 93-548). Financial support by the Dutch Cancer Society for the publication of this thesis is gratefully acknowledged.

\section{Druk:}

Datawyse Maastricht 


\section{Contents}

\section{Chapter 1}

Introduction and outline of the thesis.

General introduction

Analyzing the problem behavior

Determinants of smoking and smoking cessation 7

Changing smoking behavior 15

$\begin{array}{ll}\text { Overview of the thesis } & 19\end{array}$

Part I Cognitive determinants of readiness to change and quitting.

\section{Chapter 2}

The pros and cons of quitting, self-efficacy, and the stages of change in smoking cessation.

Introduction $\quad 24$

Method 26

Results $\quad 29$

Discussion 31

\section{Chapter 3}

Smokers in four stages of readiness to change. 35

Introduction $\quad 36$

Method 38

Results 41

Discussion $\quad 47$

\section{Chapter 4}

Self-evaluation and motivation to change: Social cognitive constructs in smoking cessation.

Introduction

Method $\quad 54$

Results $\quad 58$

Discussion 62

\section{Part II The effectiveness of computer-tailored interventions.}

\section{Chapter 5}

The development of computer-generated tailored interventions. 67

Introduction 68

Phases in the development 69

$\begin{array}{ll}\text { Conclusion and discussion } & 79\end{array}$ 


\section{Chapter 6}

Tailored interventions to communicate stage-matched information to smokers in different motivational stages.

Introduction

Method

Results

85

Discussion

\section{Chapter 7}

Long-term effectiveness of computer-generated tailored feedback in smoking cessation.

Introduction

Method

Results

Discussion

\section{Chapter 8}

Tailoring information to enhance quitting in smokers with low motivation to quit: Three basic efficacy questions.

Introduction

Method

Results

Discussion

\section{Chapter 9}

Targeting smokers with low readiness to change with tailored and nontailored self-help materials.

Introduction

Method

Results

Discussion

\section{Chapter 10}

General discussion and integration.

Introduction

Determinants of readiness to change and smoking cessation

The tailored interventions

Methodological issues

Applications of the findings

Recommendations

References

Summary

Samenvatting 


\section{Chapter 1}

\section{Introduction and outline of the thesis}

\section{General introduction}

This thesis is about smokers who have a low motivation to quit smoking, and the development and effectiveness of computer-generated tailored interventions to increase their motivation to quit.

To illustrate the necessity to motivate smokers to quit smoking, the health effects of smoking will be described. For example, a recently published study stated that about half of all cigarette smokers will eventually be killed by their habit (Doll, Peto, Wheatly, Gray \& Sutheriand, 1994). The figures undoubtedly illustrate the fact that smoking behavior constitutes a problem. To complicate matters, smoking is also highly addictive. Once a smoker becomes physically addicted to nicotine and psychologically dependent on the presumed functions of smoking, he or she tries to sustain the behavior, and attempts to quit smoking are renowned for their failure rate (Marlatt \& Gordon, 1985).

In an attempt to decrease this health problem, smokers might be encouraged to quit smoking. A part of the smoker population is interested in quitting and will benefit from a call to quit smoking. However, a substantial part of the smoker population is considered to have low motivation to quit smoking. In three representative U.S.A. samples of smokers, the proportion of smokers with low motivation to quit was about $40 \%$ (Velicer, Fava, Prochaska, Abrams, Emmons \& Pierce, 1995), while in European samples the proportion ranged from $57 \%$ in Finland (Pallonen, Fava, Salonen \& Prochas$\mathrm{ka}, 1992$ ) to about $70 \%$ in the Netherlands (Mudde, Dolders \& De Vries, 1994). This group of smokers is the population under investigation in the present thesis. A core problem in constructing messages designed to encourage these smokers to quit, is that these smokers have little interest in information on smoking or smoking cessation. Hence, an intervention especially designed to communicate health information to this group of smokers is needed.

Health education attempts to change behaviors by changing their psychological determinants. In the theoretical framework used in this thesis, behavior is determined by the readiness to change behavior (similar to the intention/goal construct) which, in turn, is determined by perceived outcomes (physical, social and self-evaluative) and self-efficacy expectations. In Part I of this thesis, the cognitive characteristics of smokers with low motivation to 
quit is studied. Their levels of psychological determinants will be compared with those of smokers who are motivated to quit and ex-smokers. Furthermore, an attempt is made to unravel the psychology of those with low motivation to quit despite knowing the detrimental effects of smoking. The relations among the determinants and between the determinants on the one hand and intention to quit and quitting behavior on the other hand are studied.

Once it is known which psycholagical determinants have to be targeted in an intervention, the contents of the intervention can be developed. In contrast with traditional health education interventions which are aimed at everyone but at no one in particular, the interventions developed in this thesis are adapted (tailored) to relevant individual characteristics. By putting foreknowledge of individuals into a computerized tailoring-system, so-called 'computer-tailored interventions' or 'tailored letters' can be produced on a large scale. In Part Il of this thesis, a methodology to develop such a tailoringsystem is outlined and several aspects of the effectiveness of the tailored intervention are tested in three large randomized field-experiments. Finally, the results are discussed and recommendations for the application of tailored interventions and possible directions for future studies are given.

\section{Analyzing the problem behavior}

\section{The ABC-planning model}

The ultimate goal of the present research is to develop effective smoking cessation interventions. A systematic approach is needed to asure that the interventions are appropriate to the specific target group, directed at relevant determinants of the behavior in this target group and implemented in the right way. The ABC-planning model differentiates three phases in the process of health education and promotion (De Vries, 1989; De Vries \& Kok, 1996). The first phase is the Analysis phase which is characterized by five steps: problem identification; analysis of the problem factors and of health action to reduce the problem; target group identification; analysis of motives of a target group to participate in a particular behavior; acces point analysis to studly how and where the target group can be reached. The second phase - Behavioral change - consists of three steps: definition of goalls and objectives; program development; program testing. The third phase Continuation - also consists of three steps: developing intersectoral collaboration; diffusion of the program; defining supportive policies. The present thesis is concerned with the first two phases. With respect to the first phase it focuses on the problem identification, on differentiating the target group of smokers according to their level of readiness to change, and on analyzing the cognitive factors related to the process of smoking cessation. With respect to the second phase, the focus is on the development and evaluation of computer-generated taillored interventions. 


\section{Health consequences and prevalence of smoking}

The relation between smoking tobacco and several health problems is fimly established. In this section, a short overview of the health problems related to smoking behavior and the prevalence of smoking behavior, will be given.

About half of all cigarette smokers will eventually be killed by their habit (Doll et al., 1994). Smoking is linked with many serious health problems such as cancer of the lip, oral cavity, pharynx, larynx, esophagus, lung, pancreas, bladder and kidney. Furthermore, smoking is associated with coronary heart disease, chronic obstructive pulmonary disease and cerebrovascular disease (Solomon \& Flynn, 1993). Moreover, smoking is the leading cause of fire death, the second leading cause of fire-related injury in the United States while it is estimated that about $14 \%$ of burn hospitalizations are cigarette-related. Car driving smokers are even reported to have $50 \%$ more motor vehicle accidents than non-smokers (Sacks \& Nelson, 1994). Moreover, smoking is number 1 among the preventable causes of death in the U.S.A. (Ginnis \& Foege, 1993). It is estimated that world-wide about 3.000.000 smokers die yearly from smoking (Peto, Lopez, Boreham, Thun \& Hearh, 1994), while in the Netherlands each year almost 30.000 smokers die from smoking tobacco (Dutch Foundation on Smoking and Health, 1995). It is clear that smoking tobacco causes serious health problems. This is even more problematic because there has been a massive worldwide increase in cigarette consumption during the past few decades (Peto et al., 1994). In the developed countries the prevalence has declined, although the consumption of tobacco per smoker has increased. For example, in the year 1967 , approximately $60 \%$ of the Dutch population between 15 and 65 years of age smoked (men $78 \%$; women $42 \%$ ). In 1995 this percentage was 35\% (men $39 \%$; women $31 \%$ ). Although this is a decline of $42 \%$, the absolute number of smokers in the Netherlands decreased by only $21 \%$ (from 5.21 million to 4.31 million). Moreover, the average number of cigarettes smokers consume a day increased by $56 \%$ (from 12.9 to 20.1 ), and while in $1967,35 \%$ of these cigarettes were hand-rolled (containing higher levels of nicotine and tar than manufactured cigarettes) $46 \%$ were in 1995. Thus, although the proportion of smokers showed a desirable downward trend, there are still many smokers who on average consume even more and heavier tobacco (Dutch Foundation on Smoking and Health, 11995). In other developed countries, similar changes in prevalence have been observed and, overall, about $41 \%$ of men and $21 \%$ of women are smokers. In developing countries, overall, $50 \%$ of men and $8 \%$ of women smoke, with the latter figure rising (Slama, 1994). In the Proceedings of the 9th World Conference on Tobacco and Health the seriousness of the problem is summarized as follows: "If the epidemic continues at the present rate, within 30 or 40 years tobacco will be killing 10 million people each year. One death every three seconds...." (Slama, 1994, p.2). 


\section{Smoking is an adddiction}

Smoking tobacco also constitutes a major problem because it is an addiction. In the international classification of psychopathology, the Diagnostic and Statistical Manual of Mental Disorders (4th ed.; American Psychiatric Association, 1994), smoking is included as a substance use disorder. The addictive properties of smoking make that smokers are trapped in physical and psychological dependence.

Plhysical dependence is caused by nicotine. According to Christen and Christen (1994) compulsive nicotine intake causes physical tolerance, tissue dependence, psychic dependence and relatively well-defined physical withdrawal symptoms. Physical dependence is considered to contribute to the experience of craving for the substance. In the same way as a shortage of water leads to thurst (craving for water), which is experienced as a compelling experience based on a bodily need, acute abstinence from nicotine leads to craving for a cigarette. Sustained abstinence (6-12 hours) leads to withdrawal symptoms which can be viewed as signs of the body being out of balance without the usual blood nicotine levels. Concluding, once a smoker is physically dependent on nicotine, the body installs a strong motive to smoke cigarettes.

Besides this physical dependence, smokers become psychologically dependent on smoking. Psychological dependence can be described in cognitive and motivational terms. According to Marlatt and Gordon (1985) craving for the substance is caused by the expectancy of the desired effects of a drug. In the cognitive view of Beck, Wright, Newman and Liese (1993) these expectancies are called addictive beliefs. Addictive beliefs are a cluster of ideas about the perceived functions of a drug and they: "... contribute to maintaining the addiction and provide the groundwork for relapse" (Beck et al., 1993; p. 38). In the theoretical model used in this thesis these expectations are referred to as negative outcome expectations of quitting smoking. Smokers not only have strong beliefs about the functions of smoking, they also use cognitive mechanisms which keep at bay a conflict between the motive to use and the motive not to use. Beck et a]. (1993) define permission giving beliefs, which are reasons to use the substance in spite of the known detrimental effects. These beliefs may be viewed as excuses or dissonance reducing beliefs (Chapman, Wong \& Smith, 1993). Furthermore, smokers seem to use attentional processes to keep threatening information away. Beck et al. (1993) speak of a "cognitiwe blockade" if attentional resources become allocated to using the drug and inhibit awareness of the delayed long-term consequences of smoking (also see Janis \& Mann, 1977). Concluding, a smoker is psychologically dependent on smoking when beliefs on the functions of smoking install a motive to smoke, while a self-preserving and bolstering cognitive state may try to keep away information on potential motives to quit (positive outcomes of quitting).

Both physical and psychological dependence contribute to the experience 
of low control over smoking, while at the same time this experience of control is essential in the motivation to quit and in smoking cessation (Marlatt \& Gordon, 1985; Mudde, Kok \& Strecher, 1995; Strecher, McEvoy DeVellis, Becker \& Rosenstock, 1986).

The present research is conducted within the realm of health education. Therefore, the research is specifically directed at influencing these psychological causes of smoking and smoking cessation.

\section{The target group}

One major reason for using the $\mathrm{ABC}$-planning model is that the effectiveness of interventions partly depends on the extent to which it takes into account the characteristics of the people who engage in the problem behavior, that is, smoking. Since many different groups can be recognized within the smoker population, the effectiveness of interventions is considered to be higher when smoking cessation interventions are directed at specific groups. Therefore, a target group has to be clearly defined and described. The stages of change model (Prochaska, DiClemente \& Norcross, 1992) - which will be used and extended in the present thesis - is useful for recognizing some relevant potential target groups with regard to smoking cessation. First, there are the ex-smokers who are in danger of relapse. Specific relapse prevention interventions might help to maintain the, until then, successful quit attempt. In the stages of change model, these ex-smokers are considered to be in the action or maintenance stage of smoking cessation. Second, there are smokers who are motivated to quit and plan to quit in the short term. Approximately $20 \%$ of the smokers in the U.S.A. (Velicer et al., 1995) and $7 \%$ of the smokers in the Netherlands (Mudde et al., 1994) are planning a quit attempt within the next 30 days. These smokers have a need for smoking cessation materials. In the stages of change model these smokers are considered to be in the preparation stage of smoking cessation. Third, there are smokers who have plans to quit somewhere in the next six months, but not (yet) within the next month. In contrast with the former group, these smokers are not yet preparing an attempt, they are just contemplating it. In the stages of change model, these smokers are considered to be in the contemplation stage of smoking cessation. In the U.S.A., this group constitutes approximately $40 \%$ of the smoker population (Velicer et al., 1995), while in the Netherlands this is $26 \%$ (Mudde et al., 1994). Fourth, there is a group of smokers who have no plans to quit in the forseeable future. They are considered to have low motivation to quit. In the U.S.A. this group constitutes approximately $40 \%$ of the smoker population (Velicer et al., 1995), while in the Netherlands the percentage is about $70 \%$ (Mudde et al., 1994). In the stages of change model, these smokers are considered to be in the precontemplation stage of smoking cessation. Figure 1.1 shows the percentages of smokers in three stages in two European and two U.S.A. samples (Etter, Perneger \& Ronchi, 1997). 


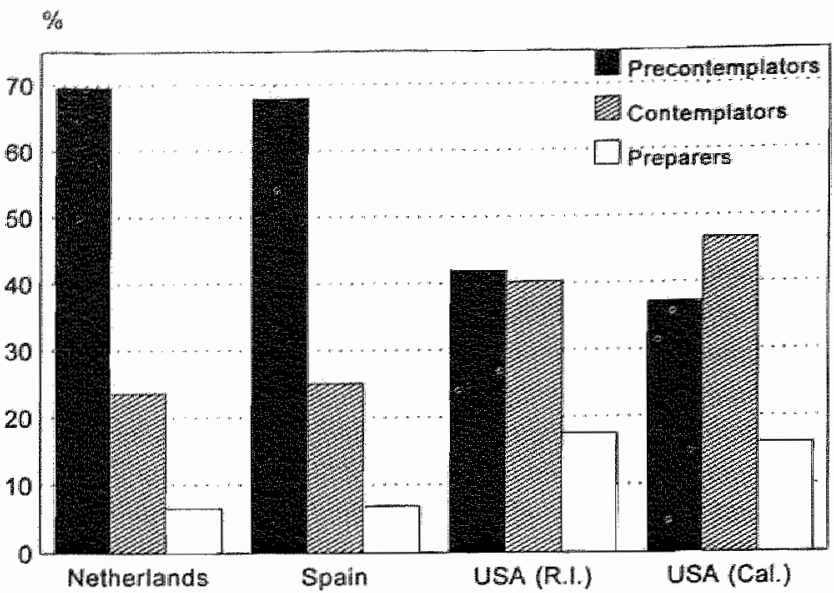

Figure 1.1 Smokers in the stages of change in four representative samples (Etter, Perneger \& Ronchi, 1997).

Thus, the potential target group of smoking cessation interventions is heterogeneous. Moreover, there are indications that the (ex-)smokers in each stage may benefit from different sorts of information with respect to smoking cessation (De Vries \& Backbier, 1994; De Vries \& Mudde, in press; DiClemente et al., 1991; Prochaska et al., 1992). Interwentions which target (ex-)smokers in one particular stage - stage-matched interventions - take into account the specific cognitive and information processing characteristics of individuals in that stage. A stage-matched smoking cessation interventions for precontemplators might take into account the findings that precontemplators see few benefits of quitting and seem to psychologically avoid information on smoking and smoking cessation (Prochaska et al., 1992; Prochaska, Velicer, Guadagnoli, Rossi \& DiClemente, 1991). Prochaska and colleagues have developed the so-called 'stage-matched manuals' of which one manual is specifically directed at those smokers with low motivation to quit. Only recently, the Dutch Foundation of Smoking and Health developed a self-help guide and a brochure in each of which one chapter is devoted to increasing the perceived benefits of quitting and making smokers aware of their psychological defenses. However, both these Dutch health education materials are mainly directed at and framed for (ex-)smokers in the other stages of change. This means that although the large group of smokers with low motivation is recognized, no health education materials are available for this group.

In conclusion, the high percentage of precontemplators in the smoker populations and the lack of matched health education materials on smoking cessation has motivated the theoretically based development of smoking cessation materials for precontemplators. This development will be described in the present thesis. Smoking cessation interventions direct the psychological determinants of cessation behavior. In Chapter 2,3 and 4 in this thesis, the psychological determinants of smoking cessation in precontemplators will be 
studied. The theoretical model which is used in these and the other chapters in this thesis will be outlined in the following paragraphs.

\section{The determinants of smoking and smoking cessation}

\section{Models of behavior and behavior change}

In health education, several theoretical models map the psychological factors which are considered to determine behavior and behavior change. For example, the Health Belief Model (HBM; Janz \& Becker, 1984), the Theory of Planned Behavior (TPB; Ajzen, 1988), the Attitude/Sociall influence/Efficacy Model (ASE; De Vries, Dijkstra \& Kuhlman, 1988), ProtectionMotivation Theory (PMT; Maddux \& Rogers, 1983) and Bandura's Social Cognitive Theory (SCT; 1986) define - and to a varying extent operationalize - the psychological determinants of behavior. Although the models partly stress different psychological factors and the operationalizations of the factors may differ greatly, three main cluster of psychological determinants can be recognized in all models (Kok, Schaalma, De Vries, Parcel \& Paulussen, 1996; Petraitis, Flay \& Miller, 1995). First, the models describe a factor which refers to perceived future outcomes. For example, certain models use constructs such as attitude (TPB), perceiwed benefits (HBM) or outcome expectations (SCT). Second, the models describe a factor which refers to social influence. For example, certain models use constructs such as social norm (TPB), extrinsic rewards (PMT) and social outcome expectations (SCT). Third, the models describe a factor which refers to the perception of control over cognitive or behavioral tasks. For example, certain models use constructs such as perceived behavioral control (TPB) and self-efficacy (SCT, ASE, PMT). In the present thesis, the Social Cognitive Theory (SCT) is used as the theoretical framework. SCT is a comprehensive psychological theory which can be used to describe psychopathology (Bandura, 1986; Bandura, 1997; Pervin, 1989), personality (Pervin, 1989), and normal psychology and behavior change (Bandura, 1986). In SCT, the three main clusters of psychological influences on behavior and behavior change are defined as: 1) physical and self-evaluative outcome expectations; 2) social outcome expectations and; 3) selfeefficacy. The concept of modeling in Bandura's theory is considered to be a determinant of outcome expectations and self-efficacy expectations. That is, people learn to expecti certain outcomes of behavior and they judge their self-efficacy partly on the basis of modeling influences.

\section{Social cognitive constructs: expected outcomes}

According to Bandura (1986), and many instrumental learning theorists, behavior is extensively regulated by its effects. In SCT, people regulate their behavior on the basis of anticipated effects of their behavior. This anticipati- 
on is referred to as outcome expectations. Thus, outcome expectations are cognitive representations of future events. The impact of expected outcomes on behavior is not primarily related to the accuracy of the predictions, but to their anticipated aversive or rewarding nature.

Bandura distinguishes three kinds of outcome expectations: physical outcomes, social outcomes and self-evaluative outcomes (Bandura, 1997). Physical outcomes refer to bodily needs and exert their power through, for example, anticipation of deprivation, satiation or pain. Sociall outcomes refer to social rewards or disaproval. Social outcomes partly exert power through their association with, for example, aversive events such as deprivation of privileges and withdrawal of love, or rewards such as the allocation of social status. Both, physical and social outcomes refer to the personal experience of external events. Self-evaluative outcomes, however, refer to aversive or rewarding experiences caused by self set internal standards. When people compare their accomplishments with their personal standards or goals, they react with negative or positive self-evaluation, depending on whether the demands of the standards or goals are met. As with physical and social outcomes, people engage in behavior which they think will lead to positive self-evaluation, and avoid behavior which they think will lead to negative self-evaluation.

Several studies show that concepts related to expected outcomes, such as valued beliefs, attitudes or the pros and cons of quitting, are related to motivation to quit and smoking behavior (De Vries et al., 1988; DiClemente et al., 1991; Dijkstra, Bakker \& De Vries, 1997; Godin, Valois, LePage \& Desharnais, 1992; Prochaska et al., 1991; Willemsen, De Vries, Van Breukelen \& Oldenburg, 1996). The more reward smokers anticipate through quitting, the more they are motivated to quit and the more they actually quit. In Chapters 2 and 3 of this thesis, the outcome expectations which are important in smoking cessation are investigated. In Chapter 4 , the focus is on self-evaluative outcome expectations and their relation with the intention to quit and smoking cessation.

\section{Social cognitive constructs: self-efficacy expectations}

Self-efficacy expectations are the judgements a person makes about his or her ability to successfully accomplish a specific task. As such, self-efficacy is a construct of control (Skinner, 1996). In SCT, behavior is not determined by actual control, but by the perception of control. According to Bandura (1986), perceived self-efficacy is a significant determinant of performance and partially operates independently of underiying skills. When a person's self-efficacy judgements with regard to a certain behavior are positive, he or she is more motivated to engage in that behavior and the chances to accomplish the related task are increased. Furthermore, self-efficacy judgements are not a belief of general personal control but they are task-specific (Bandura, 1986). 
It is increasingly recognized that selfefficacy expectations are important in health behavior (Strecher et al., 1986; De Vries et al., 1988; Bandura, 1997; Schwarzer, 1992). Health education interventions try to increase positive self-efficacy expectations. In smoking cessation the specific task or behavior is mostly formulated as "refraining from smoking". Indeed, prospective studies show that self-efficacy expectations are predictive of abstinence (Coelho, 1984; Condiotte \& Lichtenstein, 1981; De Vries \& Mudde, in press; DiClemente, 1981; Kavanagh, Pierce, Sing \& Shelley, 1993; McIntyre, Lichtenstein \& Mermelstein, 1983; Mudde et al., 1995).

\section{Operationalizations of outcome expectations and self-efficacy}

SCT does not determine the operationalisation of its constructs (Pervin, 1987). Therefore, other sources were used as the basis of the operationalisations of outcome expectations and self-efficacy in the present studies.

Outcome expectations were operationalized according to the ASE-model (De Vries \& Backbier, 1994; Willemsen et al., 1996), in which both the behavior (e.g. quitting smoking) and a consequence (e.g. better health) are formulated. Items were formulated as follows: "If I quit smoking, then...". The anticipated reward, and consequently its motivating power, is considered to be greater if someone states, for example, "If I quit smoking, my risk of lung cancer will decline very much", then in the case in which someone states "If I quit smoking, my risk of lung cancer will decline a lithle".

Self-efficacy expectations were also operationalized according to the ASEmodel (De Vries \& Backbier, 1994; Willemsen et al., 1996). First, the smoker was asked to imagine that he or she engaged in a serious quit attempt. Second, the smoker was asked to judge his or her self-efficacy with regard to refraining from smoking in a given situation. The items were formulated as follows: "Do you think you are able to refrain from smoking in situation X?" and they can be scored from "I am not at all certain that I am able to refrain from smoking" to "I am very certain that I am able to refrain from smoking". The more certain a person was that he or she would be able to refrain from smoking in that situation, the higher the self-efficacy was considered to be.

\section{The process nature of behavior change: The stages of change}

Behavior change is a process: people move from no motivation to change the unhealthy behavior to, eventually, the internalization of the healthy behavior. Several models acknowledge this explicitly. For example, the transtheoretical model (TTM; Prochaska et al., 1992), Weinstein's Precaution Adoption Process (WPA; Weinstein, 1988) Janis and Mann's Theory of Conflict (JMTC; Janis \& Mann, 1977) and Gebhardt's Health Behavior Goal Model (HBG; Gebhardt, 1997) all describe the process of behavior change. In this process, different stages or phases are described in which people have 
to engage in different tasks in order to progress to the following stage. In the present thesis, the stage of change construct from the TTM is used.

The stage of change construct (SCC) has become popular in the field of health education. Partly, this seems due to its temptingly simple and intuitively plausible nature: Instead of regarding behavior change as a discrete and clearly delineated event, behavior change is regarded as a process which takes time to develop. People transit through a series of discrete stages before they internalize a new behavior. Although $\mathrm{SCC}$ has changed over the years, most innovations are based on the following structure. In the first stage, people do not think about changing a certain behavior. These smokers are in the precontemplation stage and are considered to have low motivation to change. In the next stage, people consider changing and they actually make a decision to take action. They are considered to be in the contemplation stage. Then, people change their behavior. In this stage, called the action stage, the behavior is not yet a routine, however. Lastly, in the maintenance stage, people have internalized the new behavior and it is integrated in their lives.

A central tenet of the model is that it is normal to cycle through the stages more than once, as relapse is viewed as inherent to behavior change. By explicitly describing a state of Jow motivation to change and relapse to former behavior, the model seems to take into account human liability to err. Furthermore, by recognizing the limitations of human capability to change beliavior, the model seems to give a realistic description of behavior change.

\section{Developments of the stages of change construct}

During the last fifteen years several aspects the SCC have changed. First, the number of stages described has differed over the years. Whereas in the early model five stages were identified, precontemplation, contemplation, action, maintainence and relapse (Prochaska \& DiClemente, 1983; Prochaska, DiClemente, Velicer, Ginpil \& Norcross, 1985), later, relapse was no longer regarded as a stage but as a transition from one stage to another (DiClemente et al., 1991). Furthermore, a subgroup was identified within the contemplation stage: whereas people in contemplation are held to only contemplate behavior change, people in the preparation stage are considered to have made the decision to change in the short term, that is, they are planning to quit within the next month. Additionally, people in preparation are already changing their behavior, that is, they have made a quit attempt in the last year (DiClemente et al., 1991). A recent development with regard to $\mathrm{SCC}$ is the identification of possible subgroups within the precontemplation stage (Crittenden, Manfredi, Lacey, Warnecke \& Parsons, 1994; Dijkstra et al., 1997; Velicer, Hughes, Fava, Prochaska \& DiClemente, 1995). In Chapter 3 of this thesis, an extended model which recognizes two groups of smokers within the precontemplation stage, is investigated.

In the efforts to develop or expand the $\mathrm{SCC}$, it is especially important to 
decide, on theoretical and empirical grounds, whether a given subgroup constitutes an additional stage or "just" a subtype within the group of precontemplators. In additional stages of change, specific psychological profiles have to be discovered for each stage with specific tasks in the transition from one stage to another. Subtypes can best be described in terms of one specific psychological profile (e.g. precontemplator) but differing on other variables.

A second development in the last one and a half decades, is that the model has changed with regard to its measurement. Mostly, the stages are assessed using a few simple dichotomous questions. For example, people can be classified as being in a certain stage of smoking cessation, using the following algorithms: Smokers who are not planning to quit in the next six months are considered to be in precontemplation. Smokers who are planning to quit in the next six months are considered to be in contemplation. Smokers who are planning to quit in the next month (and have engaged in a serious quit attempt of at least 24 hours in the last twelve months) are considered to be in preparation. Ex-smokers who have refrained from smoking for no longer than 6 months are considered to be in the action stage, while ex-smokers who have refrained from smoking for more than 6 months are considered to be in the maintenance stage. Using these algorithms, people are classified as being in one particular stage of change. One major weakness of the algorithms is that the time-criteria have no theoretical base. Nevertheless, the criteria have been proven to have discriminative power, cross-sectionally (De Vries \& Backbier, 1994; Prochaska et al, 1991; Velicer, DiClemente, Prochaska \& Brandenburg, 1985), and longitudinally (DiClemente et al., 1991; Prochaska, 1991). Furthermore, the algorithms lack validity for some health behaviors. In smoking cessation, people can accurately assess whether they do or do not smoke and there is consensus about the recommended healthy behavior (i.e., not smoking at all). However, in fat intake reduction, for example, many people have a biased view of their actual fat consumption (Lechner, Brug \& De Vries, in press; Brug, 1996) and many people do not know the recommended level of the healthy behavior. Thus, the readiness to change scores of these people are based on an inaccurate assessment of their present (fat intake) behavior. Therefore, adaptations of the criteria for stage assignment have been proposed (Lechner, Brug, De Vries, Van Assema \& Mudde, in press).

The stages are regarded as refering to discrete, that is, qualitatively different psychological (precontemplation, contemplation, preparation) and behavioral (action, maintenance) states (DiClemente et al., 1991; Prochaska et al., 1992). With regard to the early stages - which are 'only" distinguished on the basis of quitting plans - it is particularly hard to decide to what extent they differ quantitatively or qualitatively. Using a discrete measure leads to distinguishing discrete stages, whereas using a quantitative measure leads to a quantitative description of stages. For example, in the early model, the stages were assessed using a 32-item questionnaire (McConnaughy, DiClemente, Prochaska \& Velicer, 1989; McConnaughy, Prochaska \& Velicer, 
1983). A high score on an item such as: "As far as I am concerned, I don"t have any problems that need to be changed", was indicative of the precontemplation stage. A high score on an item such as: "It might be worthwhile to work on my problem" was indicative of the contemplation stage. A high score on an item such as: "I am finally working on my problems", was indicative of the action stage, whereas a high score on an item such as: "I may need a boost right now to help me maintain the changes I've already made", was indicative of the maintenance stage. This measure led to a quantitative score for being in precontemplation, contemplation, action and maintenance. Rollnick, Heather, Gold \& Hall (1992) used a 12-item Readiness to Change Questionnaire assessing the first three stages, modeled on the original 32 -item questionnaire, and found that only $40 \%$ of the participants under study could be classified as being in one specific stage. Furthermore, in a recent study, the notion of the stages of change refering to discrete qualitatively different stages was not supported using this 12-item questionnaire (Budd \& Rollnick, 1996): the data seemed to support a continuous measure of 'readiness to change', rather then a model describing discrete stages. Finally, Prochaska, Norcross, Fowler, Follick \& Abrams (1992) measured stages of change with the original 32-item measure and described their population under study in quantitative terms: "Participants... progressed from being initially more in the contemplation stage to being more in the action stage of change" (p.42). In conclusion, whether we find discrete stages or a continuous process depends on what we measure.

\section{Operationalization of the stages of change construct}

In the present studies the stages of change are assessed using simple algorithms. The model deviates from the original model (DiClemente et al., 1991; Prochaska et al., 1992) on two important aspects.

First, the criterion to be assigned to the preparation stage differs. Whereas in the original model smokers in preparation 'are planning to quit within the next month" and "have made a serious quit attempt in the last year', in the currently used stage model the latter criterion is dropped. Apart from the fact that smokers are now more consistently assigned to a stage on the basis of their intention to quit, this adjustment eliminates a llaw of the original model. Using the original criteria, contemplators who have not engaged in a quit attempt in the last year can never enter the preparation stage. This means that when these contemplators quit smoking, they will have to skip the preparation stage. This contradicts a central tenet of the stage modell.

Second, the present model deviates with regard to the precontemplation stage. In the original model, smokers are assigned to the precontemplation stage if they are not planning to quit within the next six months. Using this criterion, about $70 \%$ of smokers are in the precontemplation stage in the Netherlands (Mudde et al., 1994). In order to study this probably heterogenous group, an earlier study investigated whether subgroups could be 
recognized within this stage (Dijkstra et al., 1997). On the basis of this study, the large group of smokers who were not planning to quit within the next six months was divided into: 1) immotives, who were not planning to quit within the next five years, and; 2) precontemplators, who were planning to quit within the next five years. Hence, the currently used stage model classified smokers (except in Chapter 2) into four instead of into three stages of increasing readiness to change: immotives, precontemplators, contemplators and preparers. In Chapter 3 of this thesis, the model is replicated and further investigated. The four stages are considered to refer to relevant cutoff points on a measure of readiness to change. Ex-smokers can be classified as being in action or in maintenance (Table 1.1).

Table 1.1

Stages of change: Relewant cut-off points on a measure of readiness to change.

\begin{tabular}{lll}
\hline Behavior & Stages & Criterion \\
\hline $\begin{array}{l}\text { Refrained } \\
\text { from smoking }\end{array}$ & Maintenance & Refrained from smoking for more than six months \\
& Action & Refrained from smoking for less than six monws \\
Snoking & Preparation & Planning to quit within the nex month \\
& Contemplation & Planning to quit within the next six months \\
& Precontemplation & Planning to quit within the next year \\
& Planning to quit within the next five years \\
& Planning to quit sometime in the future but not yet \\
& within the next five years \\
& Not planning to quit ever, only planning to cur down \\
& Not planning to quit ever and not planning to cut down \\
\hline
\end{tabular}

\section{The integrated model}

The theoretical model used in the present studies is depicted in Figure 1.2. Outcome expectations and self-efficacy are considered to determine readiness to change and behavior. Furthermore, readiness to change is considered to determine behavior.

Outcome expectations influence readiness to change and behavior through their energizing power (motivation; Bandura, 1986). Under certain conditions (Bagozzi, 1992), this energizing power is converted to a state of readiness to change: the more valued the outcome expectations, the more energy (higher motivation) and the higher the readiness to change.

With regard to self-efficacy, four routes to behavior can be distinguished. Notably, self-efficacy judgements by themselves do not energize people. The mere fact that a person judges that he or she is able to accomplish a certain task has no motivating power. First, self-efficacy determines behavior 
through its influence on the expected positive outcomes (Bandura, 1986). That is, self-efficacy expectations influence motivation by influencing the awailability of anticipated reward. In the case of a person having low selfefficacy, this implies that the chances of succes are lowered and, hence, that the anticipated experience of reward through successful accomplishment becomes less sure. On the other hand, when a person has high self-efficacy, this implies that the chances of success are increased and, hence, that the anticipated rewards through successful accomplishment will be achieved. Furthermore, during the execution of the behavior, higher self-efficacy leads to more effort, more persistence or perseverance to accomplish the task, for example, refraining from smoking (Bandura, 1986).

Second, self-efficacy determines behavior through its influence on the expected negative outcomes. For example, a smoker who smokes for relaxation, might expect a negative outcome from quitting because he or she loses a means to relax. This expected negative outcome is considered to inhibit behavior change. However, if the smoker develops high self-efficacy with regard to alternative ways to relax, the impact of the expected negative outcome on behavior change might decline.

Third, self-efficacy determines behavior by its influence on the readiness to change. Self-efficacy is considered to determine the actions people intent to take: people do not choose to engage in tasks they judge to be beyond their ability (Bandura, 1986). As such, self-efficacy determines (goal-) intentions or, in the present model, readiness to change.

Fourth, self-efficacy determines behavior through its direct influence on behavior. To the extent that self-efficacy judgements refer to actual efficacy or skills to engage in a certain task, self-efficacy determines behavior directly.

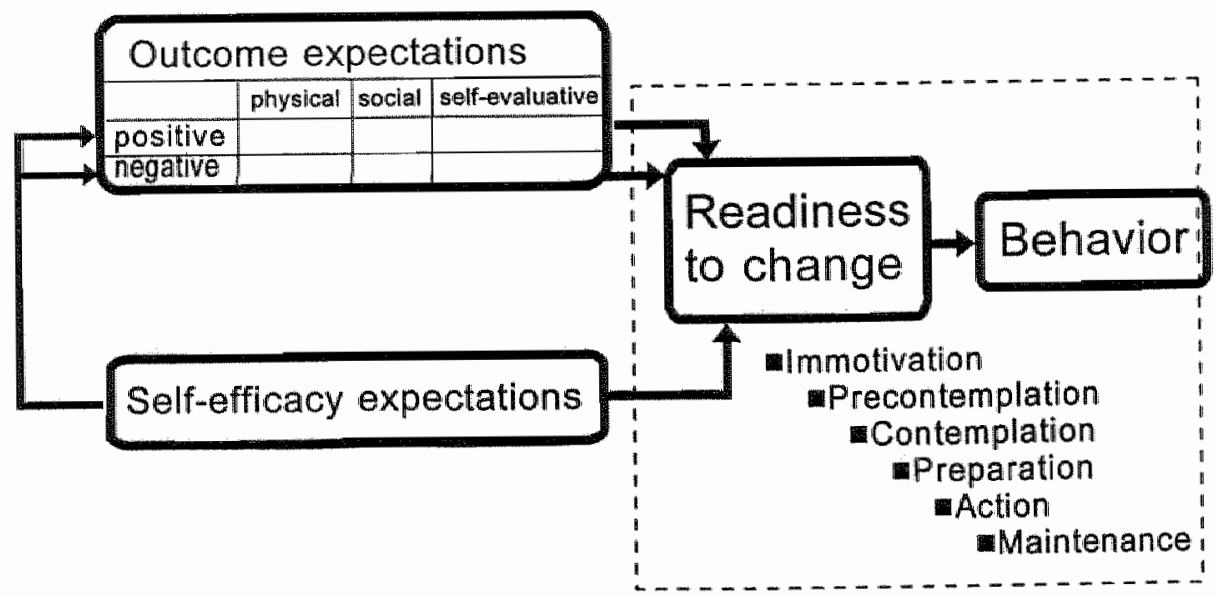

Figure 1.2 The theoretical model 


\section{Changing smoking behavior}

\section{The objectives of the intervention}

In the second phase of the $\mathrm{ABC}$-planning model, the intervention is developed and tested. First, the objectives of the intervention must be defined. Although the eventual aim of smoking cessation interwentions is to make smokers quit smoking, in the stage of change model the goal is to stimulate smokers in the process of smoking cessation. Therefore, the goall of the interventions needs to be stage transition. For smokers with low readiness to quit, the goal is increasing the readiness to quit, rather than making them stop smoking. For contemplators, the goal is to stimulate transition to the preparation stage and for preparers the goal is to initiate and support a quit attempt. Thus, the goal of the intervention is to stimulate forward stage transition. From the theoretical model described above (Figure 1.2), it follows that interventions will have to increase the positive outcome expectations of behavior change, decrease the negative outcome expectations and increase positive expectations of self-efficacy. Hence, these are the general intervention objectives in this thesis. However, it might be that the intervention objectives differ for interventions aimed at smokers in different stages. This is one of the questions to be answered in the studies in this thesis. Regardless of the answer, the determinants will be changed in the following ways.

Positive outcomes are increased by offering information which is relevant to install or strengthen the expectation of an outcome. For example, information on the fact that quitting smoking decreases the risk of lung cancer refers to a potential reward of quitting (depending on an individual's or society's value system). Negative outcomes are decreased by offering information which moderates the aversive character of the expected negative outcome. For example, expected withdrawal symptoms in smoking cessation are negative outcome expectations, in that they make people anticipate an aversive experience in the case of quitting. Moderating information might address the temporal character of withdrawal symptoms.

With regard to influencing self-efficacy expectations, the aim would be to increase self-efficacy expectations with regard to a specific task. For example, a specific task could be "refraining from smoking when I feel stressed". First, information on skills to be used to accomplish the task could be offered. It is thought that the more effective ways (ex-)smokers have to cope with difficult situations, the higher the self-efficacy. Second, information on how successful others are will influence self-efficacy expectations. For example, when similar others can cope with a certain situation, a smoker might conclude that he or she should also be able to cope successfully. Third, self-efficacy might be influenced positively by reinterpreting physical experiences, such as withdrawal symptoms. For example, reinterpreting withdrawal symptoms as "signs of the body"s fight to become independent of 
nicotine" might change the perception of the chances of accomplishing the task successfully.

\section{Tailored self-help interventions}

Self-help smoking cessation interventions are designed to change the cognitive factors outlined above. However, the effectiveness of self-help smoking cessation interventions can be regarded as relatively low: point prevalence quit rates at 1-year follow-up tend to range between 10 and $20 \%$ (Schwarz, 1987; also see Cohen et al. 1989). Therefore, increasing the effectiveness of self-help interventions in smoking cessation is an important issue. Within that framework, a relatively new sort of self-help intervention has emerged: the tailored intervention. With foreknowledge of a person or a group of persons, the contents of tailored interventions are adapted to the relevant characteristics measured of an individual. In order to adapt the contents of an intervention on several features, a computerized system is needed to produce them (De Vries, Willemsen, Brug, Dijkstra \& Berben, 1995; Skinner, Siegfried, Kegler \& Strecher, 1993; Velicer et al., 1993). In Chapter 5 of this thesis, a methodology to develop such a computerized tailoring system is presented.

Because computer-tailored interventions - or tailored letters - are a recent innovation, few data on their effectiveness are available. The following short overview of the different aspects of the effectiveness of tailored interventions is structured according to the treatment evaluation strategies proposed by Kazdin (1986). Results from tailored interventions with regard to health behaviors other than smoking cessation are included.

The first treatment evaluation strategy is called the "treatment package strategy". This strategy evaluates the effect of a particular treatment as that treatment is ordinarily used. Kazdin: "To rule out the influence of change as a function of historical events, maturation or spontaneous remission of the dysfunction, repeated testing and other threats to internal validity, a notreatment or waiting-list control condition is included in the design" (p. 25, 1986). With regard to the effectiveness of tailored self-help interventions, a search of the literature reveals that a single tailored intervention can indeed lead to more changes in behavior than no information, after four to six months (Campbell, DeVellis, Strecher, Ammerman, DeVellis \& Sandler, 1994; Strecher et al., 1994). To test whether our newly developed tailored interventions meet this minimum criterion, their effects are compared to the effects in a no-information control condition, in Chapter 6,7 and 8 of this thesis.

The second treatment evaluation strategy is called the "dismantling treatment strategy". The dismantling strategy consists of analyzing the components of a given treatment package. Kazdin: "To dismantle treatment, individual components are eliminated or isolated from the treatment" (p.25, 1986). Most tailored interventions seem to include at least two main tailoring 
working mechanisms, adaptation of the content information and personal feedback. Skinner, Strecher \& Hospers (1994) studied whether one working mechanism, adaptation of the content information, had a cognitive/behavioral changing impact on its own, that is, apart from personal feedback. They compared general messages to tallored messages which: "... contained individual-specific information but were framed to sound as though they were written for general audiences" (p. 45). The results show that the tailored messages still led to significantly more forward stage movement than the general messages, in black and low-income women. Thus, the adaptation of the content information had a cognitive/behavioral changing impact on its own, that is, apart from personal feedback. In the present thesis, the 'given treament package' consisted of the single tailored intervention containing information on outcomes of quitting and self-efficacy enhancing information. In Chapter 6, this tailored intervention is compared to a tailored intervention containing only information on possible expected outcomes and a tailored intervention containing only self-efficacy enhancing information.

The third treatment evaluation strategy is called the "constructive treatment strategy". The constructive treatment strategy is used in developing interventions by adding components that may enhance outcome. According to Kazdin this strategy asks the question: "What can be added to treatment to make it more effective?" (p.26, 1986). Brug, Steenhuis, Van Assema, Glanz \& De Vries (in press) compared a condition which offered dietary feedback with a condition offering dietary feedback plus psycho-social feedback. The data showed that the addition of psycho-social feedback had no surplus value. In a recent study, Kreuter \& Strecher (1996) compared a condition offering health risk feedback (Health Risk Appraisal; HRA) with a condition offering such feedback in combination with psycho-social information aimed at supporting behavioral changes. Several health behaviors were assessed and selectively addressed in the interventions. The results showed that the addition of the psycho-social information increased the effectiveness of the HRA significantly. Curry, Wagner \& Grothaus (1991) compared the effects of a tailored smoking cessation letter, a price incentive (and a secret gift), the combination of both and no information. They reported that the condition offering the tailored letter only led to significantly more smoking cessation than all three other conditions. Thus, the addition of the price incentive to the tailored letter actually reduced the effect. Chapter 8 of this thesis investigates whether the addition of a self-help guide (containing 46 pages of information) to a tailored intervention enhances the effectiveness. Furthermore, we could regard tailoring of information as the addition of a new element to the content information of smoking cessation interventions. In an ultimate test to assess whether adding this tailoring element to content information leads to higher effectiveness, a tailored intervention must be compared to a non-tailored intervention with the same lay-out, length and presentation, and addressing the same topics. The results of two such studies can be summarized as follows. Compared to a non-tailored intervention, a tailored interven- 
tion led to significanily more quirters, but only in a group of lighter smokers (Strecher et al. 1994), and to a lesser fat intake (especially in the group of "risk consumers'), but not to larger changes in fruit and vegetable consumption (Brug, Steenhuis, Van Assema \& De Vries, 1996; also see Campbell et al., 1994). In Chapter 8 of this thesis, our single tailored letter is compared to a non-tailored look-alike letter.

The fourth treatment evaluation strategy is called the "parametric treatment "trategy". This strategy refers to altering specific aspects of a treatment to determine how to maximize therapeutic change. According to Kazdin, the components of treatment are not withdrawn (dismantling approach) or added (constructive approach). Kazdin: "Essentially, parametric research focuses on refining a particular technique. In parametric research, variations often are made along quantitative dimensions by presenting more or less of a given sort of treatment" (p. 27, 1986). Brug, Glanz, Van Assema \& Kok (1996) compared a tailored intervention consisting of one leiter with dietary feedback with a tailored intervention consisting of two subsequent and related letter's with dietary feedback. The data showed that the addition of another tailored letter increased the effectiveness. The studies reported in Chapters 8 and 9 of this thesis investigate whether a tailored intervention consisting of three consecutive and related tailored letters is more effective than a single tailored intervention.

The fifth treatment evaluation strategy is called the "comparative treatment strategy". In this strategy, two or more treatments are compared. Kazdin: "The question addressed by this strategy is which treatment is better (or best) among various alternatives for a particular problem" (p. 27, 1986). Curry, McBride, Grothaus, Louie and Wagner (1995) evaluated the incremental effects of using: 1) a self-help booklet alone; 2) the self-help booklet with computer-generated personalized feedback and; 3) the self-help booklet, personalized feedback, and outreach telephone counseling. The personalized feedback was not more effective than the self-help booklet, in their nonvolunteer sample of smokers. The telephone counseling condition was more effective than the other conditions, only at the short term follow-up (three months). Prochaska. DiClemente, Velicer and Rossi (1993) compared: 1) standardized smoking cessation manuals; 2) smoking cessation manuals matched to an individual's readiness to change; 3 ) the matched manuals plus personalized feedback reports and; 4) matched manuals, personalized feedback reports plus telephone counseling. The condition offering the matched manuals plus personalized feedback reports was the most effective after 18 months while the matched manuals were more effective than the standardized manuals. Thus, taking into account certain characteristics of an individual, as in the matched manuals and personalized feedback reports, led to higher effectiveness. In Chapter 9 of this thesis, three smoking cessation self-help interventions are compared: 1) tailored intervention consisting of three subsequent and corresponding tailored letters; 2) a single tailored letter; 3) a standardized self-hellp guide. 
From the above, it is clear that with regard to tallored smoking cessation interventions, few data are available. Nevertheless, the results are promising and they justify further investigation and development of tailored interventions.

\section{Intervention effects in smokers with low motivation to quit}

Curry et al. (1995) and Prochaska et al. (1993) reported on the effects of their interventions in smokers who were not planning to quit within the next six months. Curry et al. showed that their most extensive condition - rhe self-help booklet, personalized feedback, and outreach telephone counseling was the most effective in this group of smokers. Although no contrast analyses were reported, comparing the condition offering the self-help booklet with computer-generated personalized feedback, the percentage of quitters was tripled. Prochaska et al. found the contrary: their condition offering the matched manuals plus three tailored letters was more effective than the condition which added counseling to these letters. No significant differences were reported, however. Finally, Pallonen et al. (1994) compared stage-matched manuals to a no-intervention control condition. The matched manuals led to significantly more quitting in smokers with low readiness to quit at one year follow-up but not at two years follow-up. Thus, few results of interventions in smokers who are not planning to quit within the next six months are available and the results which are available show mixed outcomes.

\section{Overview of the thesis}

In Part I of this thesis, the cognitive determinants of readiness to change and smoking cessation are investigated in three studies (Table 1.2).

In Chapter 2 (study I) a cross-sectional analysis of smokers and exsmokers in five stages of readiness to change is presented. Smokers in precontemplation, contemplation, preparation, action and maintainence are compared on expected positive and negative outcomes (pros and cons of quitting) and self-efficacy expectations.

In Chapter 3 (study II), a stage model which classifies smokers into four instead of into three early smoking cessation stages is investigated (Dijkstra et al., 1997). The following four groups of smokers with increasing readiness to change are compared on their levels of cognitive determinants: immotives, precontemplators, contemplators and preparers. Furthermore, to study whether stage of readiness to change is a determinant of smoking cessation, it is investigated to what extent stage membership at $\mathrm{T} 1$ is predictive of smoking cessation after three and after fourteen months.

In Chapter 4 (study III), the Self-Evaluation-Motivation model (SEM), which was developed on the basis of the SCT, is presented and tested. The model describes the relation between physical, social and self-evaluative 
outcome expectations, on the one hand, and intention to quit and smoking cessation, on the other hand. It is investigated to what extent self-evaluative outcome expectations determine intention and behavior and what cognitive factors, in turn, dertermine self-evaluation.

In Part II of this thesis, the development of tailored interventions and the testing in three randomized field experiments (reported in four studies) of different sorts of tailored self-help interventions is presented (Table 1.2).

In Chapter 5 (study IV) of this thesis, a three-phase model to develop tailored interventions is outlined. The aim is to give an idea of the potential of tailored interventions, to increase insight in the typical tailoring working mechanisms to be included in tailored interventions and to show how tailored interventions can be theoretically and empirically based.

Chapter 6 (study $\mathrm{V}$ ) focuses on the extent to which smokers in the four different stages need different sorts of information in order to encourage quitting. In a 4 (Stages) X 4 (Conditions)-design, smokers are randomly allocated to one of four tailored intervention conditions offering: 1) outcome information; 2) self-efficacy enhancing information; 3) both sorts of information or; 4) no information.

Chapter 7 (study VI) studies whether tailored letters consisting of only 5 to 7 pages of information on smoking cessation are still effective after fourteen months. This study presents the long-term data from the study presented in Chapter 6 .

Chapter 8 (study VII) investigates in a randomized field triall $(N=752)$ : 1$)$ whether a single tailored letter is more effective than a non-tailored lookalike; 2) whether the addition of a 46-page smoking cessation self-help guide to a tailored intervention enhances the effectiveness and; 3) whether three subsequent and related tailored letters (multiple tailored intervention) mailed to smokers with one month intervals are more effective than a single tailored letter. This research sample consisted only of smokers with low readiness to change (i.e. immotives and precontemplators).

Chapter 9 (study VIII) presents the results of another randomized field trial $(\mathrm{N}=843)$. It studies: 1) whether a multiple tailored intervention, a single tailored intervention and at standardized self-help guide are more effective than no information; 2) whether the tailored interventions are more effective than the standardized self-help guide and; 3) whether the multiple tailored intervention is more effective than the single tailored intervention. Again, this research sample consisted only of smokers with low readiness to change (i.e. immotives and precontemplators).

In Chapter 10 , the results on the cognitive determinants and the effectiveness of the tailored interventions are discussed and integrated and some methodological issues are addressed. Finally, reconmendations for health education interventions directed at people with low readiness to change and possible directions for future studies are given. 


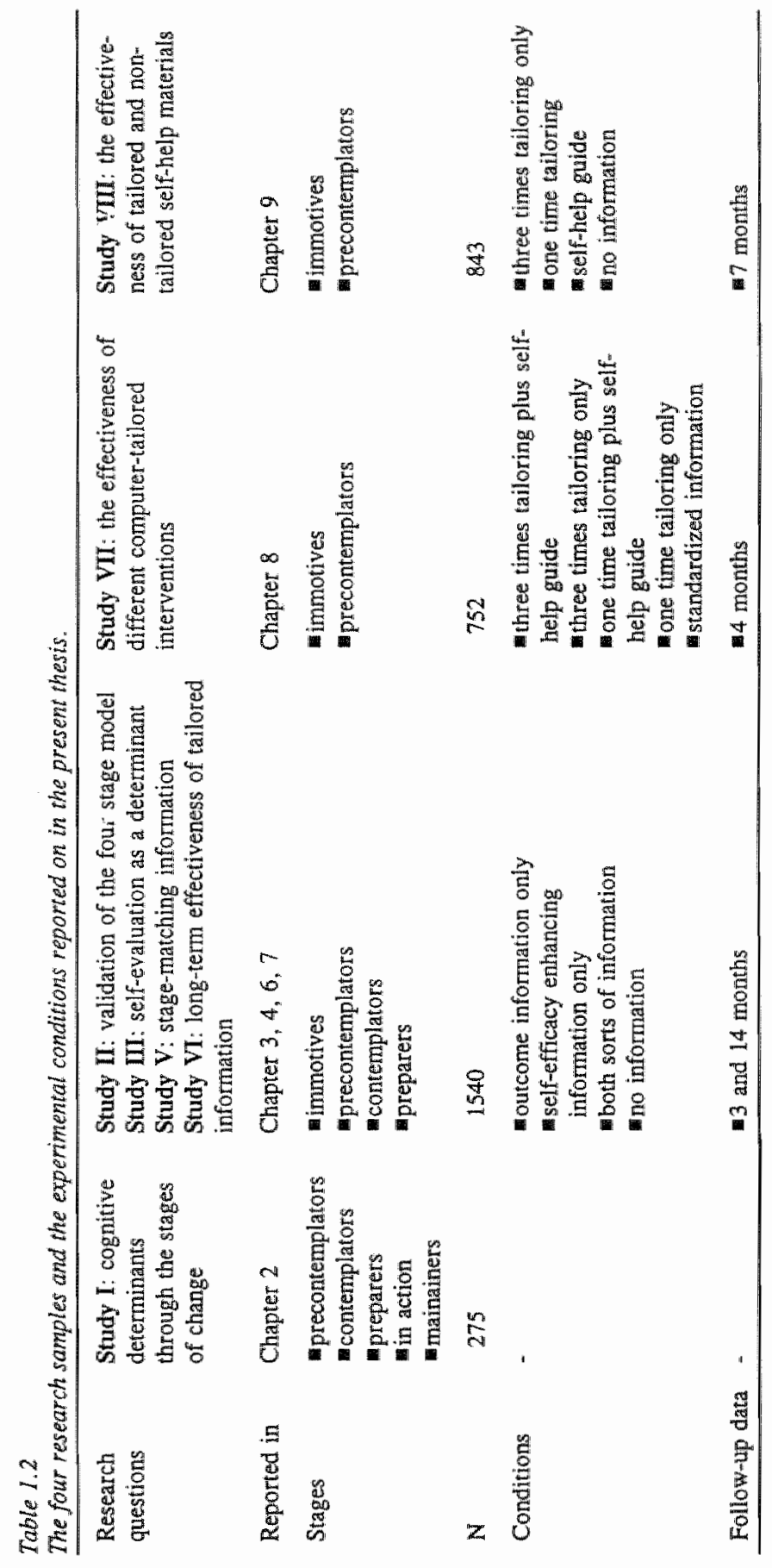




\section{Part I}

\section{Cognitive determinants of readiness to change and quitting}




\title{
Chapter 2
}

\section{The pros and cons of quitting, self-efficacy, and the stages of change in smoking cessation ${ }^{1}$}

\begin{abstract}
In the Netherlands $34 \%$ of the population smoke and $70 \%$ of these smokers are not planning to quit. The lower percentages in the U.S. population seem to reflect a difference in smoking culture. The present study analyses the pros and cons of quitting and self-efficacy expectation in the five Stages of Change in the Dutch populations. The results are compared to the pattern of the pros and cons of smoking and self-efficacy expectations found in U.S. samples. The data show the hypothesized pattern: in the first two stages the expected positive outcomes of quitting discriminated better between the stages than self-efficacy whereas for later stages self-efficacy was the better discriminator. The present study shows the stage typology is applicable to the Dutch smokers population and that the pattern of the pros, cons and selfefficacy are very similar to the pattern found in U.S. populations.
\end{abstract}

Published as: Dijkstra, A. De Vries, H. \& Bakker, M. (1996). The pros and cons of quitumg, setr.

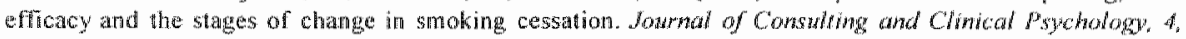
$758-765$. 


\section{Introduction}

Smoking cessation outcome has traditionally been viewed as a dichotomy. The goal of most interventions is to make people stop smoking now and for ever. However, the effects of interventions on overall smoking rates in the population are small partly due to the fact that only a minority of smokers are motivated to quit in the short term. In the Netherlands $34 \%$ of the population smoked in 1991 (Dutch Foundation on Smoking and Health, 1992), while approximately $70 \%$ of these smokers were not planning to quit in the near future (six months; Mudde, Dolders \& De Vries, 1994). In the U.S. these percentages are 26\% (Jarvis, 1994) and 37\% (Velicer, Rossi, Ruggiero \& Prochaska, 1994) respectively. The large percentage of smokers in the Netherlands who are not planning to quit indicates a need to develop interwentions for this group.

Prochaska and DiClemente (1983) described smoking cessation as a process instead of a dichotomous outcome. Smokers are thought to move through different motivational stages from no motivation to quit to maintaining the new behavior (Prochaska, DiClemente \& Norcross, 1992). They describe five stages of change and define the first three stages through a measure of intention to quit and the last two stages by (non-smoking) behavior.

Prochaska (1991) showed that interventions, developed for people who are ready to change behavior, have lower efficacy for people who are not ready for action. After an intervention quit rates among smokers in the precontemplation, contemplation and preparation stage were $20 \%, 38 \%$ and $67 \%$ respectively. Since both the intention to quit and the success rate of the intervention differ for the three groups, it is plausible that the effectiveness of interventions can be improved by developing specific interventions aimed at each motivational stage. These interventions have to stimulate the different stage transitions as adequately as possible and lead people from no motivation to quit to the internalization of the new behavior.

The type of information given in a particular stage will depend on the determinants responsible for positive and negative stage transition. Ideally, changes in determinants of behavior change should be assessed when people move through the stages from no plans to quit to actually changing their behavior. In the present study the problem will be addressed exploratively by assessing how smokers in the different stages differ on relevant determinants of behavior change.

In their transtheoretic model, Prochaska and his colleagues (Prochaska, 1994; Prochaska et al., 1994) describe a specific pattern of the Decisional Balance constructs, the pros and cons of changing, through the stages. This pattern has been verified with regard to changes in several problem behaviors including smoking. Precontemplators anticipate more cons than pros with regard to changing the problem behavior, whereas the pros and cons are more in balance in the contemplation stage. In the action stage people anticipate more pros than cons. In comparing smoking behavior with the other problem 
behaviors, the pros and cons of quitting are considered to be complementary to the cons and pros of smoking respectively (Prochaska et al., 1994).

Furthermore, the transtheoretic model posits that people in the action and maintenance stage have higher self-efficacy than people in the precontemplation stage (Prochaska \& DiClemente, 1984; Prochaska, Velicer, Guadagnoli, Rossi \& DiClemente, 1991). It is not yet clear to what extent these data can also be generalized to smokers in the Nethertands. The findings of De Vries and Backbier (1994), however, were similar to the findings with the transtheoretic model, despite the use of different cognitive constructs and a Dutch sample of smokers. De Vries and Backbier (1994) found, in pregnant women, that the attitude towards quitting, measured as a composite of the pros and cons of quitting, was negative among precontemplators, but was positive among contemplators and subjects in the action stage. Self-efficacy expectations were found to be low for both precontemplators and contemplators but were significantly higher among people in the action stage. Furthermore they reported that people in the action stage experienced more social support than people in the precontemplation stage. The resultant pattern for the three stage groups was referred to as the $\varnothing$-pattern (Figure 2.1) with the diagonal representing experienced social support. The findings of Prochaska et al. (1991) and Velicer, DiClemente, Prochaska and Brandenburg (1985) with regard to the cons of smoking and the findings of DiClemente, Prochaska and Gibertini (1985) and Prochaska et al. (1991) with regard to self-efficacy expectations resemble this pattern. Based on this pattern, De Vries \& Backbier (1994) suggest that smokers in the different stages of change may need different sorts of information in order to enhance stage transition.

The first goal of the present study was to compare smokers in all five stages of change on the pros and cons of quitting and self-efficacy cognitions. A search of the literature revealed no such study including the preparation stage. Participants in all stages of change were measured at one moment in time and a cross-sectional comparison was made. The second goal was to compare the patterns of these determinants in the stages of change in our Dutch population with the patterns found in populations in the U.S. Finally, it was of interest how the pros and cons of quitting in the present study compared to the pros and cons of smoking used in the transtheoretic model (Velicer et al., 1985). Based on the findings reported above, it was expected that people in the precontemplation stage would score lower on the pros of quitting than people in the contemplation stage, preparation stage and action stage. Furthermore, people in the precontemplation and contemplation stage were not expected to differ in self-efficacy expectations but they were expected to have lower self-efficacy expectations than people in the action and maintenance stage. 


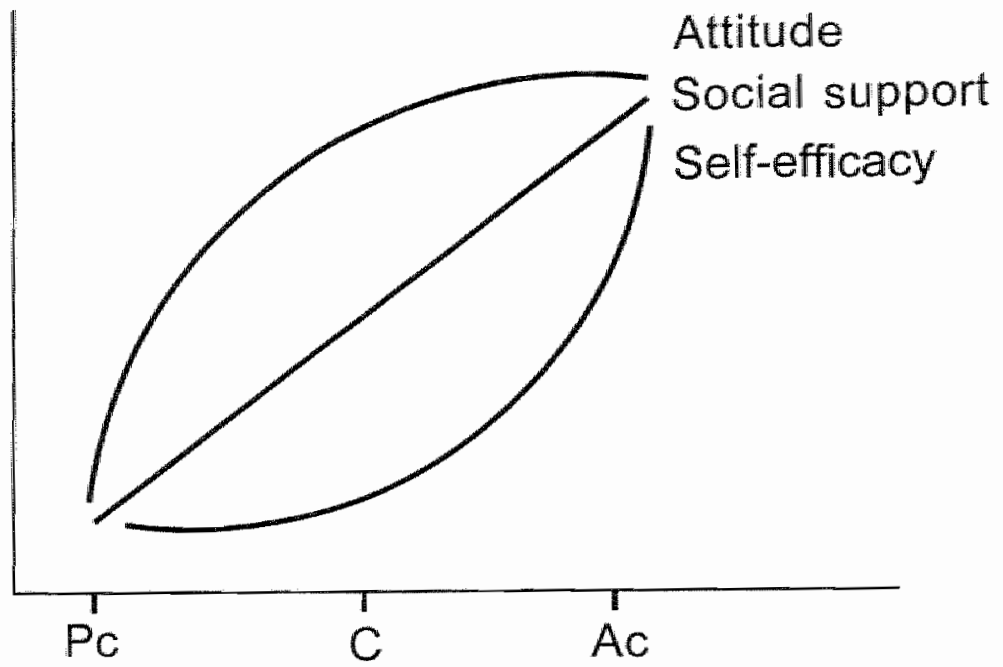

Figure 2.I The D-pattern; attitude, social support, self-efficacy and the Srages of Change (De Vries \& Backbier, 1994). Pc=precontemplation, $C=$ contemplation, $A c=$ action.

\section{Method}

\section{Participants and procedure}

Subjects representing the five stages of change were recruited by different advertisements in a local newspaper. To avoid association with smoking cessation and hence a selection bias, smokers in the precontemplation stage were asked to fill out a questionnaire about motives for smoking and in return they were offered a small gift. In the advertisements designed to recruit people in the remaining four stages of change, people were asked to fill out a questionnaire about motives to quit smoking. In return they could request a self-help manual.

People were asked to phone the university in order to register. Then they were sent the questionnaire with a request to return it in the pre-paid envelope provided within two weeks. In total 275 participants returned the questionnaire (response rates were between $80 \%$ and $90 \%$ for the different stage groups). The respondents were divided into the five stages of change having the following characteristics.

Precontemplation. Of the 121 participants in this stage $60.3 \%$ were female; average age, 39.7 years. They smoked 22.6 cigarettes a day and had smoked for 23.6 years.

Contemplation. Of the 29 participants $44.8 \%$ were female; average age, 40 years. They smoked 23.2 cigarettes a day and had smoked for 21 years.

Preparation. Of the 34 participants $32.4 \%$ were female; average age, 40.3 
years. They smoked 23.3 cigarettes a day and had smoked for 22.7 years.

Action. Of the 34 participants $50 \%$ were female; average age, 44.6 years. This group had quit for an average of 2.7 months.

Maintenance. Of the 57 participants in this stage $56.1 \%$ were female; average age, 44.3 years. This group had quit for an average of 13 months. Data on the quantity of cigarettes smoked, the duration of smoking and nicotine dependence were not gathered in both groups of ex-smokers.

\section{Table 2.I}

\section{Definitions of the Stages of Change.}

Precontemplation Has been smoking for the past 24 hours and is not planning to quit within the next six months.

Contemplation

Has been smoking for the past 24 hours and is planning to quit within the next six months but not yet within the next month.

Preparation

Has been smoking for the past 24 hours and is planning to quit within the next montla.

Action

Has not been smoking for the past 24 hours but not longer than six months.

Maintenance

Has not been smoking for the past 6 months.

\section{Measures}

The questionnaire assessed smoking behavior, demographics, stage of change, expected outcomes and self-efficacy with regard to behavior change. On the basis of the social cognitive theory (Bandura, 1986) and the attitude measure used by De Vries and Backbier (1994), 35 items on outcome expectations were gathered to compose scales for pros of quitting and the cons of quitting. Most items were stated in terms of: "If I quit smoking......", referring to the expectation of a certain outcome (Bandura, 1986). The items assessed physical, social, self-evaluative and monetary outcomes of quitting and could be scored from 0 (not expecting a certain outcome) to 3 (strong expectation of the outcome)(De Vries \& Backbier, 1994).

For all 35 items together Cronbach's alpha was 85. Factor analysis suggested two meaningful factors; the pros of quitting (expected positive outcomes) and the cons of quitting (expected negative outcomes). The pros of quitting subscale consisted of 25 items with a Cronbach's alpha of 89 . All these items referred to what people may expect to gain if they quit smoking. For example: "If I quit smoking, my chances of getting lung cancer will decline", "If I quit smoking, the inconvenience for the people around me lessens" and "I am ashamed that I have to smoke". The cons of quitting subscale consisted of 10 items $(\alpha=73)$. All these items referred to what people may expect to lose if they quit smoking. For example: "If I quit 
smoking, I will find it harder to relax" and "If I quit smoking, I will experience withdrawal symptoms".

Scale scores of the pros and cons were computed by adding the item scores. Correlation between the pros of quitting scale and the cons of quitting scale was .13. (corrected for attenuation .15). In accordance with Prochaska et al. (1994), the pros and cons of quitting were considered to be complementary to the cons and pros of smoking respectively, as they are reported in the literature.

Self-efficacy expectations were measured by 25 items on a 7 -point scale referring to how difficult smokers thought it would be to refrain from smoking (De Vries \& Backbier, 1994; Mudde, Kok \& Strecher, 1995) in social situations (with friends in a cafe, when someone offers you a cigarette), emotional situations (when you feel tense, when you feel depressed) and habitual/addictive situations (when you desire a cigarette, when you are experiencing withdrawal symptoms). It was thought people assess the difficulty of a certain task by judging their own ability with regard to the situational demands. Relevant situations were assessed by interviews with smokers and studying the literature (Condiotte \& Lichtenstein, 1981; De Vries \& Backbier, 1994; Velicer, DiClemente, Rossi \& Prochaska, 1990). Items could be scored from "I find it very difficult" $(-3)$ to "I find it very easy" $(+3)(\alpha=.97)$. Item scores were summed to form the scale scores.

Stages of change were assessed by the algorithms in Table 2.1. The typology of DiClemente et al. (1991) for the precontemplation, contemplation and preparation stage was used with one exception. In order to simplify the stage algorithm, data on whether or not smokers had made a 24-hr quit attempt in the past year (DiClemente et al., 1991) were not used in the definition of the preparation stage. These smokers were defined only by "I am planning to quit within the next month" (Snow, Prochaska \& Rossi, 1992).

Heaviness of smoking was measured by the Fagerström Test for Nicotine Dependence (Heatherton, Kozlowski, Frecker \& Fagerström, 1991) which assesses smoking habits: How much do you smoke? How soon after awaking? Is it hard to smoke not in public places? Do you smoke when you are ill? Do you smoke more in the morning and which cigarette is most difficult to give up? The minimum possible score was 0 , the maximum 10. Cronbach's alpha was. 71 . This is a satisfactory figure in comparison with the findings of Heatherton et al. and Pomerleau, Carton, Lutzke, Flessland and Pomerleau (1994).

Demographics measured were sex, age and education level which was categorized as low, medium or high level of education. 


\section{Results}

\section{Demographics and smoking status}

The five stage groups did not differ on level of education and mean age. With regard to sex distribution the groups did differ $[X 2(4, n=275)=9.7, p<$.05]; in the precontemplation stage more women were included than in the other stage groups. For all variables Sex $x$ Stage analyses were computed. If this interaction was significant $(p<.05)$, the analysis was stratified by sex. If the interaction was not significant, the term was removed and if there was no main effect of sex, this factor was removed completely.

First, the three groups of smokers were compared on their smoking habits (Table 2.2). No Sex $x$ Stage interaction was found on the three variables. With regard to Fagerström scores and quantity of cigarettes smoked, there was no main effect of sex and the three stage groups did not differ on these variables either. With respect to the duration of smoking, a main effect of sex was found $[\mathrm{F}(3,180)=7.11, \mathrm{p}<.01]$. Men had smoked for a longer time than women; 25.3 and 21.0 years respectively. This same analysis (thus correcting for a main effect of sex) revealed no main effect of stage.

People in the action stage had quit smoking for an average of 2.7 months $(\mathrm{SD}=1.5)$ and people in the maintenance stage 13 months $(\mathrm{SD}=3.8)$.

\section{Table 2.2}

Means and standard deviations on three measures of smoking behavior in the three smoker groups.

\begin{tabular}{llll}
\hline Measure & Precontemplation & Contemplation & Preparation \\
\hline Fagerström & & & \\
M & 5.0 & 5.2 & 5.3 \\
SD & 2.7 & 2.3 & 2.5 \\
Cigarettes/day & & & \\
M & 22.6 & 23.2 & 23.3 \\
SD & 8.0 & 9.2 & 11.8 \\
Years smoked & & & \\
M & 23.9 & 20.6 & 21.8 \\
SD & 11.3 & 9.0 & 9.6 \\
\hline
\end{tabular}

\section{Psycho-social determinants}

Figure 2.2 depicts the standardized T-score patterns on pros and cons scales and the self-efficacy scale. Table 2.3 shows the standardized T-scores. T-test contrasts were computed to identify differences between the stages.

With regard to the pros and cons of quitting and self-efficacy, no Sex $x$ Stage interaction was found. A main effect of sex was only found with regard 
to the cons of quitting: women expected more cons of quitting than men $[\mathrm{F}(4,270)=5.17, \mathrm{p}<05]$. This same analysis (thus correcting for a main effect of sex) revealed a significant main effect of stage $[F(4,270)=5.58, p<.001]$. People in the maintenance stage reported fewer cons of quitting than people in the first three stages $[t(270)=4.54, p<.001]$.

The pros scale differed significantly between the stages $[F(4,270)=16,66$ $p<.0011$. Smokers in the contemplation stage expected more pros of quitting than smokers in the precontemplation stage $[t(270)=3.54, p<.001]$. Smokers in the preparation stage again scored higher than smokers in the contemplation stage $[\mathrm{t}(270)=2.21, \mathrm{p}<.05]$. People in the action stage anticipated fewer pros of quitting than smokers in the preparation stage $[\mathrm{t}(270)=-1.99, \mathrm{p}<.05]$.

Table 2.3

Standardized $T$ scores (Mean=50, SD=10) of pros and cons of quitting and selfefficacy in the stage groups.

\begin{tabular}{lcccccl}
\hline $\begin{array}{c}\text { Stage } \\
\mathrm{n}\end{array}$ & $\mathrm{Pc}$ & $\mathrm{C}$ & $\mathrm{Pr}$ & $\mathrm{Ac}$ & $\mathrm{Mt}$ & $\begin{array}{l}\text { Tukey-HSD } \\
\text { contrasts }\end{array}$ \\
\hline Pros & 121 & 29 & 34 & 34 & 57 & \\
M & & & & & & \\
SD & 45.2 & 51.8 & 56.9 & 52.5 & 53.6 & $\mathrm{Pc}<\mathrm{C}, \mathrm{Pr}, \mathrm{Ac}$, \\
Cons & 9.7 & 9.2 & 8.0 & 8.4 & 8.4 & $\mathrm{Mt}$ \\
M & 51.2 & 53.4 & 52.0 & 48.9 & 45.1 & $\mathrm{Pc}, \mathrm{C}, \mathrm{Pr}, \mathrm{Ac}>$ \\
SD & 10.0 & 11.6 & 11.8 & 8.2 & 6.6 & $\mathrm{Mt}$ \\
Self-eff. & & & & & & \\
M & 45.2 & 46.4 & 47.4 & 53.9 & 61.1 & $\mathrm{Pc}, \mathrm{C}, \mathrm{Pr}<\mathrm{Ac}<$ \\
SD & 7.4 & 5.3 & 7.5 & 9.0 & 8.9 & $\mathrm{Mt}$ \\
\hline
\end{tabular}

Note. $\mathrm{P} c=$ Precontemplation, $\mathrm{C}=$ Contemplation, $\mathrm{Pr}=$ Preparation, Ac=Action, $\mathrm{Mt}=$ Maintenance. $\mathrm{Pc}>\mathrm{C}$; the mean score of $\mathrm{Pc}$ is significantly higher than that of $\mathrm{C}$.

Data on self-efficacy of four participants were missing. Among the various stages significant differences on self-efficacy scores were found $[\mathrm{F}(4,266)=44.45, \mathrm{p}<.001]$. People in the action stage found it easier to refrain from smoking than people in the preparation stage $[\mathrm{t}(266)=3.42, \mathrm{p}<.001]$, while people in the maintenance stage again found it easier to stay abstinent than people in the action stage $[\mathrm{t}(266)=4.22, \mathrm{p}<.001]$. 


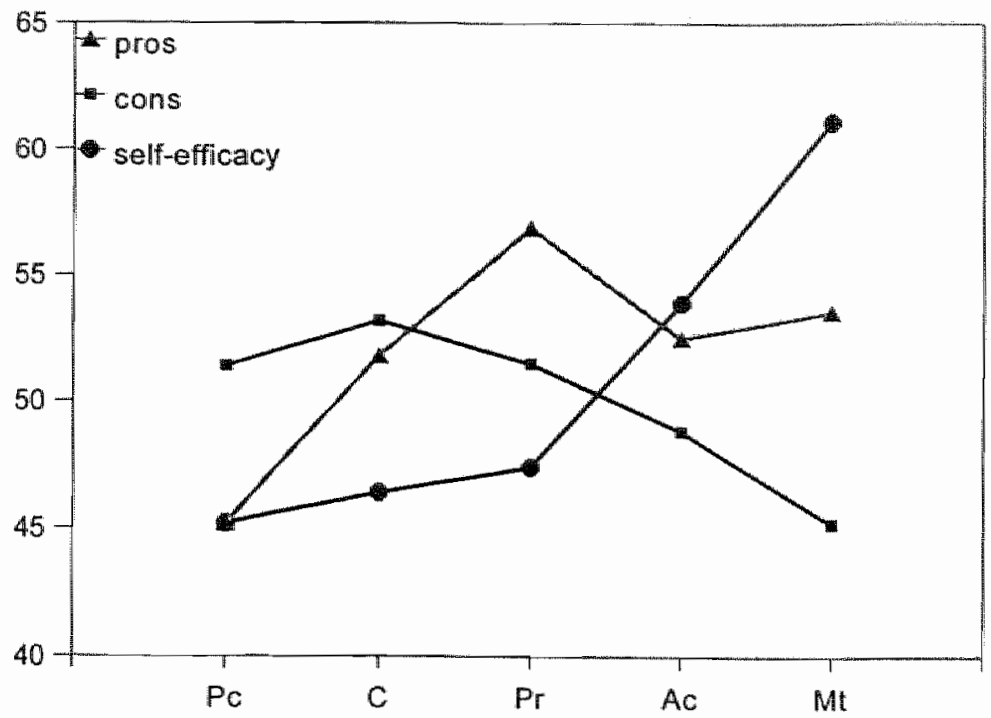

Figure 2.2 Standardized T-score patterns (Mean $=50, S D=10$ ) of the pros and cons of quitting, self-efficacy and the Stages of Change. $P_{C}=$ precontemplation, $C=$ contemplation, $P r=$ preparation, $A c=a c t i o n, M t=$ maintenance.

\section{Discussion}

The present study supports the applicability of the stage typology for Dutch smokers. People in the different stages differed on expected outcomes and self-efficacy. The fact that in the Netherlands $34 \%$ of the population smoke and $70 \%$ of these smokers are not planning to quit within six months, could have resulted in qualitatively different findings with regard to stage characteristics. However, the findings are comparable to findings in U.S populations. The present study showed that smokers in the contemplation stage anticipated more pros of quitting than smokers in the precontemplation stage whereas they did not differ in self-efficacy expectations. The respondents in the action and maintenance stage did not anticipate more pros of quitting than those in the preparation stage while they did have higher selfefficacy expectations. It is concluded that the findings of Prochaska et al. (1991) and Velicer et al. (1985) with regard to the cons of smoking and the findings of DiClemente et al. (1985) and Prochaska et al. (1991) with regard to self-efficacy expectations, as depicted in the $\varnothing$-pattern, were replicated (see Figure 2.2).

With regard to the pattern of the pros and cons of quitting in the stages, the findings were similar to the pattern found with the Decisional Balance constructs. Prochaska (1994) studied the pros and cons of changing the behavior (complementary to the cons and pros of not changing respectively) in 12 health behaviors including smoking. He discovered, based on cross- 
sectional designs, two principles of progressing from precontemplation to action:

The strong principle states that progression from precontemplation to action is a function of approximately a I standard deviation increase in the pros of a healh behavior change. The weak principle states that progression from precontemplation to action is a function of approximately a $1 / 2$ standard deviation decrease in the cons of a health behavior change (Prochaska, 1994. p. 1).

Apart from his longitudinal interpretation of the results, the present data show the weak and the strong principle: The maximum difference within the first four stages (precontemplation, contemplation, preparation and action) on the pros of quitting, was 1,2 standard deviation and the maximum difference within these stages on the cons of quitting was 0,5 standard deviation (see table 3). The findings with the Decisional Balance constructs were replicated using a different theoretical framework and a different operationalization.

Participants in the action and maintenance stage reported less pros of quitting than participants in the preparation stage. Velicer et al. (1985) and Prochaska and DiClemente (1984) also found that ex-smokers reported less reasons for quitting. It might be that people in the action stage, because they are deprived of smoking, face a difficult time and may not be experiencing the full positive consequences of quitting. Another explanation is that among the people in the action stage, a subgroup exists which may relapse in the near future. It is conceivable that these potential relapsers are characterized by anticipating less pros of quitting resulting in a lower group mean in the action stage with respect to the pros of quitting. Velicer et al. (1985), however, suggest that in the action and maintenance stage both pros and cons are of little importance or rellevance. Pros of quitting seem to drop in salience due to a decrease in importance.

When the present results are compared to the results of Snow et al. (1992) and DiClemente et al. (1991), who also define the preparation stage separateIy from the contemplation stage, some differences arise. With regard to the rcasons for smoking (pros of smoking/cons of quitting), Diclemente et all. found that smokers in the first three stages did differ: the more smokers intend to quit, the less they report reasons for smoking. This differential finding could be due to the larger power of DiClemente et al.'s study. With regard to the reasons for quitting (cons of smoking/pros of quitting), the results are consistent: the more smokers intend to quit, the more reasons for quitting they report.

In the present study, smokers in the preparation stage did not report higher selfeefficacy than precontemplators. In contrast, both Snow et al. (1992) and Diclemente et al. (1991) reported that contemplators did have significantly higher self-efficacy than precontemplators, and smokers in the preparation stage in DiClemente et al.'s study had higher self-efficacy expectations than 
smokers in the contemplation stage. Several explanations for this difference are possible. First, the difference could be explained by a different sample of smokers. Snow et al. measured the determinants and stages in smokers who were misusing or had misused alcohol. Second, because of a rather low response rate (e.g. $48 \%$ ) in Snow et al.'s study, the difference could be due to response bias. Third, DiClemente et al. employed a different recruitment procedure than that used in the present study; smokers were asked to test selfhelp manuals and were offered money for retuming the questionnaire. Fourth, the difference could be due to a lack of power of the present study. Fith, in the present study self-efficacy was operationalized as perceived difficulty of a task instead of the confidence in the ability to perform a task. Sixth, a difference in smoking culture may exist between our sample and those of DiClemente et al. and Snow et al., due to the strong anti-tobacco culture and lower smoking rates in the U.S. For example, precontemplators in the U.S, may present a group of 'hard core' smokers with no intention to quit in spite of increasing social pressure, because of relatively low self-efficacy expectations.

In the present study, the respondents in the action and maintenance stage had a higher self-efficacy than those in the preparation and contemplation stage with regard to quitting. This is not surprising since self-efficacy is strongly influenced by performing the behavior, and people in the action and maintenance stage are by definition performing the non-smoking behavior successfully.

Similar to the findings of Snow et al. (1992), more women were represented in the precontemplation stage than in the other stages. This overrepresentation of women could reflect a selection bias; precontemplating women would have more interest in joining an investigation than precontemplating men. Another explanation is that the population of smokers consists of more precontemplating women than men. Two studies in the U.S. suggest this pattern. Sorensen and Pechacek (1987) and Blake et al. (1989) reported that men were more likely to intend quitting than women. Women were more likely to report intending to cut down on the number of cigarettes they smoked. In the present study these women would be classified as precontemplators.

With regard to the Fagerström scores, no difference was found between smokers in the different stages. DiClemente et all. (1991), however, found that smokers who were in the preparation stage had lower scores than smokers in the other stages. Apart from the fact that in the present study a 6 -item scale was used instead of a 8-item scale, the difference could be due to the differential definition of smokers in the preparation stage: Using a criterion like 'a quit attempt in the last year" may select smokers who are more actively preparing a quit attempt and hence score lower on the nicotine dependence measure.

Based on the present data, we tentatively suggest that interventions would have to result in the following psychological changes. Precontemplators need to become more aware of the pros of quitting to be able to move to the 
contemplation stage. Contemplators need to become more aware of the pros of quitting and need a higher self-efficacy to move to preparation. People in the preparation stage may need interventions which enhance self-efficacy. For subjects in the action stage to move to maintenance, they need to keep up their level of self-efficacy.

More research, however, is needed to support these suggestions since they are based on cross-sectional research. Both, longitudinal and experimental studies are needed to verify the influence of the pros and cons of quitting and self-efficacy expectations on stage transition and the implications for interventions. Finally, it is of great interest to investigate the pattern of determinants through the stages in other cultures and in other health behaviors and addictive behaviors such as alcoholism, cannabis use and the use of hard drugs. 


\title{
Chapter 3
}

\section{Smokers in four stages of readiness to change ${ }^{\natural}$.}

\begin{abstract}
Smoking cessation is increasingly considered to be a process rather then a discrete event. Several stage models which stress the process nature of behavior change have been developed in the last one and a half decades. In the present study, a stage model was investigated which categorizes smokers in four groups with increasing readiness to change: immotives, precontemplators, contemplators and preparers.

Smokers from the general population were recruited to participate in an investigation on smoking cessation interventions. At pre-test, the anticipated positive and negative outcomes of quitting, perceived self-efficacy and smoking behavior were assessed. Three and fourteen months after the pretest, the follow-up measurements were conducted.

The cross-sectional results showed that smokers in the four stages differed from each other on the factors pertaining to the positive outcomes and perceived self-efficacy. No differences between the four stages were detected concerning the number of cigarettes smoked a day, the nicotine dependence score and number of years smoked. The longitudinal results showed that the stage of readiness to change at $\mathrm{Tl}$ was highly predictive of quitting at both follow-ups. It is concluded that this stage model. which divided smokers into four stages with increasing readiness to change, was supported.
\end{abstract}

'This chapter will be published as: Dijksta, A., Rojackers, J, \& De Vrics, H. (in press\}. Smokers in con stages of readiness to change. Addictive Behaniors. 


\section{Introduction}

Smoking cessation outcome has traditionally been viewed as a dichotomy. In the last one and a half decade, however, smoking cessation is increasingly described as a process: smokers become motivated to change, they decide to change and, finally, they manage to change. Several models have been developed which recognize stages in behavior change. For example, Weinstein (1988) distinguished five stages in the Precaution Adoption Process, from becoming aware of the hazards of an unhealthy behavior to the adoption of the healthy behavior. Gebhart (1997) developed four motivational stages in the Health Behavior Goal Madel. De Vries and Backbier (1994) described changes in attitudes, social influence and self-efficacy through motivational stages in their ASE-model, while Prochaska, DiClemente and Norcross (1992) defined a stages of change construct within their transtheoretical model. In the present article, a stage concept is used which is similar to that of Prochas$\mathrm{ka}$ and colleagues (1992), but which categorizes smokers into four instead of into three stages witl increasing readiness to change. In the model of Prochaska and colleagues (1992), smokers who are not planning to quit within the next six months are regarded as being in one discrete stage. In the present model, two stages are recognized within this group of smokers: immotives and precontemplators (Dijkstra, Bakker \& De Vries, 1997). Hence, smokers in four stages with increasing readiness to change were distinguished: immotives, precontemplators, contemplators and preparers. Immotives plan never to quit or are planning to quit sometime in the future (but not within the next five years). In an earlier study (Dijkstra et al., 1997), they specifically anticipated fewer long-term health consequences of smoking and they reported lower levels of self-evaluation than precontemplators, who are planning to quit within the next five years (but not within the next six months). Although precontemplators wish to quit, they have a distal, though concrete, quitting plan. Immotives are thought to be "stuck", whereas precontemplators are thought to be "on their way" to contemplation. Similarly, Velicer, Hughes, Fava, Prochaska, \& DiClemente (1995) searched for subtypes within the group of smokers who are not planning to quit with in the next six months and they reported on a subtype which was: "...clearly ready to move to the next stage" (p. 310; also see Crittenden, Manfredi, Lacey, Warnecke \& Parsons, 1994). Thus, precontemplators can transit to the contemplation stage, in which they start to process information on smoking and smoking cessation. Contemplators may transit to the preparation stage in which they make a decision to change their behavior in the short term (DiClemente et al, 1991). The algorithms used to categorize smokers in the four stages are considered to refer to relevant cut-off points on a measure of readiness to change. Readiness to change is considered to be closely related to the concept of intention (Budd \& Rollnick, 1996; Suton, 1996). Finally, smokers who quit smoking and are still actively coping with the urge to smoke are considered to be in action. 
One step in the development of a stage construct is the cross-sectional comparison of groups of smokers with differing readiness to change (De Vries \& Backbier, 1994; DiClemente et al, 1991; Dijkstra, De Vries \& Bakker, 1996). That is, when smokers in two different stages do not differ on central psychological constructs, there is litule reason to distinguish between them. Furthermore, in the development of a stage construct, the predictive validity of the stages is of importance. That is, stage assignment at one point in time should be predictive of smoking cessation activity measured at a later point in time with smokers closer to the action stage being more active (DiClemente et al., 1991).

The present study used the social cognitive theory (sct) of Bandura (1986) as the theoretical framework. Sct defines two central psychological determinants of (goal-) intentions and behavior: outcome expectations and perceived self-efficacy. It was expected that the stages used in the present study could be distinguished according to the scores on the operationalizations of outcome expectations and perceived self-efficacy. Bandura recognizes three sorts of outcome expectations: physical, social and self-evaluative outcome expectations. This distinction was empirically supported (De Vries et al., 1994; Dijkstra et al., 1997; Dijkstra, De Vries \& Roijackers, in press-a). Furthermore, self-efficacy expectations with regard to different sorts of situations may be distinguished. Earlier studies found at least two factors: self-efficacy with regard to refraining from smoking in social situations and self-efficacy with regard to refraining from smoking in emotional situations (Dijkstra et al., in press-a; Mudde, Kok \& Strecher, 1995; also see Velicer, DiClemente, Rossi $\&$ Prochaska, 1990). The self-help smoking cessation intervention which was used in this study was developed to change these cognitive determinants of smoking cessation.

The overall goal of the present study was to investigate the stage model by replicating and expanding the findings of Dijkstra et al. (1997), using a different and larger sample of smokers and adding longitudinal analyses. First, the four stages of readiness to change were explored by a cross-sectional comparison of the groups. The groups were compared on demographics, smoking behavior and on factors within the positive and negative outcome expectations and perceived self-efficacy. It was expected that the groups of smokers would differ on their perceptions of outcomes and self-efficacy expectations. Second, using longitudinal data, it was tested to what extent the stages of readiness to change were predictive of quitting behavior. It was expected that, three and fourteen months after an intervention, preparers would be more active than contemplators, contemplators would be more active than precontemplators and precontemplators would be more active than immotives. 


\section{Method}

\section{Recruitement}

Smokers were recruited by advertisements in local newspapers throughout the Netherlands. Two groups of smokers were explicitly invited to participate in this study: smokers who were planning to quit within the following six months and smokers who were not planning to quit within the following six months. They were asked to volunteer for a research project on minimal interventions for smoking cessation. The self-help intervention materials were mailed to the smoker and consisted of five to seven pages of individually tailored information on the outcomes of quitting smoking, the skills which can be used in quitting smoking or both sorts of information (detailed results on the effectiveness are reported elsewhere: Dijkstra, De Vries \& Roijackers, 1996). Subjects participating in all measurements were offered the opportunity of 10 bonus prizes amounting to $\$ 100$.

\section{Questionnaire}

The pre-test questionnaire assessed smoking behavior, readiness to change, expected outcomes with regard to behavior change, perceived self-efficacy and demographics.

The items in the scales assessing the pros and cons of quitting (positive and negative outcome expectations, respectively) were validated in an earlier study (Dijkstra et al., 1996). The present items assessed the anticipated physical, social, self-evaluative and monetary outcomes of quitting and could be scored from "not sure" or "not expecting a certain outcome" $(0)$ to a "strong expectation of the outcome" (3). The fifteen items in the pros-ofquitting scale referred to the positive consequences of behavior change (Cronbach's alpha of .87; see Table 3.3). The six items of the cons-of-quitting scale referred to the negative consequences of behavior change, and consisted of six items ( $\alpha=58$; If I quit smoking...: I will gain weight; it will be harder to socialize; I will miss a means to relax; I will miss the smoker's image; I will be bored more often; I will experience withdrawal symptoms).

Self-efficacy was measured using three different operationalizations (Mudde et al., 1995). First, a seven-item composite measure assessed self-efficacy expectations with regard to refraining from smoking in social and emotional situations $(\alpha=94)$. The items were measured on a 7 -point scale and could be scored from "not sure at all that I can stop myself smoking" $(-3)$ to "very sure that I can stop myself smoking" $(+3)$. Second, an overall item with the same format as the seven items above, assessed self-efficacy with regard to "all possible situations". Third, an overall item assessed perceived difficulty to refrain from smoking in "all possible situations". It could be scored from "very easy" (+3) to "very difficult" (-3). 
Readiness to change was assessed by confronting smokers with different goals with regard to smoking cessation. Smokers were classified as preparers if they agreed on a plan to quit within the next month and as contemplators if they agreed on a plan to quit within the next six months (but not within the next month; DiClemente et al., 1991; Dijkstra et al., 1996; Snow, Prochaska \& Rossi, 1992). Within the group of smokers who did not agree with both plans, two groups were distinguished (Dijkstra et al., 1997): smokers who agreed on a plan to quit within the next five years (precontemplators) and smokers who did not agree on the five year plan but agreed on a plan to quit sometime in the future or had plans never to quit (immotives) (Table 3.1).

Heaviness of smoking was measured by the Fagerström Test for Nicotine Dependence (FTND) (Heatherton, Kozlowski, Frecker \& Fagerström, 1991) which assesses smoking habits: How much do you smoke? How soon after awaking? Is it hard not to smoke in public places? Do you smoke when you are ill? Do you smoke more in the morning and which cigarette is most difficult to give up? The minimum possible score was 0 , the maximum 10 $(\alpha=.71)$.

Smoking behavior was measured by asking smokers how many years they had smoked, the number of cigarettes they smoked a day and whether they had made a quit attempt in the previous 12 months.

Demographics measured were gender, age and level of education. Smokers were categorized in one of four age groups according to the quartiles in the present sample (16-30 years; $31-38$ years; $39-48$ years; $49-82$ years). Level of education was categorized as low, medium, or high level of education. In the diverse schooling system in the Netherlands, the low level of education refers to vocational training, the medium level to advanced vocational training, and the high level to college/university training.

Quitting behavior was measured at three (T2) and fourteen (T3) months follow-up. First, quitting behavior was assessed with the question: "Have you smoked during the last seven days? (even one puff)" (yes/no). Second, quitting behavior was measured with the question: "Are you engaged in a quit attempt at this moment?" (yes/no). Third, a retrospective report of quitting behavior was assessed with the question: "Have you engaged in a quit attempt of at least 24-hours since the last questionnaire?" (yes/no).

\section{Procedure}

Smokers who phoned the university to register were sent the pre-test questionnaire together with a pre-paid return envelope. Of the 1733 questionnaires sent, 1606 were returned, 60 of which either had more than one page which was not completed or was completed by a subject who only smoked a pipe or cigars, leaving 1540 questionnaires $(88.9 \%)$ at T1. Subjects were randomly assigned to one of four smoking cessation intervention conditions stratified by readiness to change (low: immotives and precontemplators; high: contemplators and preparers). In the present study, data from the pre-test (T1) 
and the three (T2) and fourteen months (T3) follow-up were used. At the three months follow-up, $71.2 \%$ of the T1-participants returned the questionnaire and at fourteen months $64 \%$ returned the questionnaire.

Table 3.1

Categorization of smokers in four stages.

\begin{tabular}{|c|c|c|}
\hline Group & $\mathrm{N}$ & Criterion \\
\hline Preparers $(16.6 \%)$ & 255 & Planning to quit within the next month \\
\hline Contemplators $(24.3 \%)$ & 374 & $\begin{array}{l}\text { Planning to quit within the next six } \\
\text { months }\end{array}$ \\
\hline \multirow[t]{2}{*}{ Precontemplators $(26.6 \%)$} & 203 & Planning to quit within the next year \\
\hline & 206 & $\begin{array}{l}\text { Planning to quit within the next five } \\
\text { years }\end{array}$ \\
\hline \multirow[t]{3}{*}{ Imnotives $(32.6 \%)$} & 262 & $\begin{array}{l}\text { Planning to quit sometime in the future } \\
\text { but not yet within the next five years }\end{array}$ \\
\hline & 148 & $\begin{array}{l}\text { Planning to never quit, but planning to } \\
\text { cut down }\end{array}$ \\
\hline & 92 & $\begin{array}{l}\text { Planning to never quit and not planning } \\
\text { to cut down }\end{array}$ \\
\hline
\end{tabular}

\section{Statisticall analyses}

Three sets of analyses were performed, using cross-sectional data. First, the four groups of smokers, immotives, precontemplators, contemplators and preparers, were compared on demographics and a quit attempt within the last twelve months using chi-square analyses. Second, principal component analysis with varimax rotation was used to search for factors within the items of the pros of quitting, the cons of quitting and the self-efficacy expectations. Third, the four groups of smokers were compared on factors within the pros and cons of quitting and self-efficacy expectations, on the two overall selfefficacy items and on three measures of smoking behavior using MANOVAs. In the first step, for each dependent variable the interactions of each of the demographic variables (Gender, Age, Education) with the Stage was tested. In the case of a significant interaction $(p<.05)$, the analyses were stratified according to the demographic variable. Otherwise, the interaction was removed from the analysis. In the second step demographics were left in the analyses as covariates but were removed whenever there was no main effect ( $>$-05) on the dependent variable. Whenever a main effect of Stage was significant, contrasts between the groups of smokers were computed, using the MANOVA contrast procedure. 
Finally, longitudinal analyses were performed to investigate whether readiness to change at $\mathrm{Tl}$ was predictive of quitting behavior measured after three and after fourteen months. Three binary outeome measures were used: 1) seven-day quit; 2) present quit attempt; 3) 24-hour quit (the retrospective report of a 24-hour quit attempt). After entering Condition and the demographic factors as covariates, Stage was entered as a categorical variable in logistic regression analyses to predict quitting behavior. We were specifically interested in whether smokers in one stage differed significantly from smokers in the adjacent stages. Hence, it was tested whether precontemplators had quit more than immotives, whether contemplators had quit more than precontemplators and whether preparers had quit more than contemplators. In each comparison, the latter group functioned as the reference group.

\section{Results}

\section{Demographics, past quitting behavior and smoking behavior}

Smokers in the four groups were compared on demographics, a quit attempt in the previous twelve months and smoking behavior (Table 3.2). There was no significant difference $\left[X^{2}(3,1543)=6.76, p>05\right]$ with regard to gender distribution. The groups did differ significantly $\left[X^{2}(6,1535)=18.26\right.$, $\mathrm{p}<.01]$ regarding educational level, indicating fewer low educated participants in precontemplation and contemplation, fewer high educated participants in both motivated groups and fewer mediun educated participants in both low motivated groups. Age distribution also showed a significant difference $\left[X^{2}(9,1535)=68.12, p<.001\right]$ between the groups. There were fewer participants from the youngest group (16-30 years) and more participants from the age group 39-48 years in contemplation and preparation, fewer participants from the age group 31-38 years in the immotive group and fewer participants from the oldest group (49-82 years) in precontemplation.

There was a significant difference with regard to having made a quit attempt in the previous 1.2 months $\left[X^{2}(3,1542)=263.71, p<.001\right]$ : Only $19.7 \%$ of the immotives had made a quit attempt, while $71.9 \%$ of the snokers in preparation had made a quit attempt.

With regard to present smoking behavior the following was found. No differences between the four groups were detected conceming the number of cigarettes snoked a day $[F(3,1524)=.56, p>05]$, the FTND score $[F(3,1485)=1.44, \quad p>05]$ and number of years smoked $[F(3,1436)=1.09$, $\mathrm{p}>051$. 
Table 3.2

Demographics and smoking behavior variables.

\begin{tabular}{|c|c|c|c|c|c|}
\hline \multirow[b]{2}{*}{ Variables } & \multicolumn{4}{|c|}{ Stages of readiness to change } & \multirow[b]{2}{*}{ sign. } \\
\hline & $\begin{array}{l}\text { Immo- } \\
\text { tives } \\
n=502\end{array}$ & $\begin{array}{l}\text { Precontem- } \\
\text { plators } \\
\mathrm{n}=409\end{array}$ & $\begin{array}{l}\text { Contempla- } \\
\text { tors } \\
n=374\end{array}$ & $\begin{array}{l}\text { Prepa- } \\
\text { rers } \\
n=255\end{array}$ & \\
\hline $\begin{array}{l}\text { Gender }(\%) \\
\text { female } \\
\text { male }\end{array}$ & $\begin{array}{l}57 \\
43\end{array}$ & $\begin{array}{l}63.5 \\
36.5\end{array}$ & $\begin{array}{l}59.9 \\
40.1\end{array}$ & $\begin{array}{l}54.3 \\
45.7\end{array}$ & ns \\
\hline $\begin{array}{l}\text { Education(\%) } \\
\text { low } \\
\text { medium } \\
\text { high }\end{array}$ & $\begin{array}{l}24.4 \\
37.7 \\
37.9\end{array}$ & $\begin{array}{l}20.2 \\
40.9 \\
38.9\end{array}$ & $\begin{array}{l}19.4 \\
49.5 \\
31.2\end{array}$ & $\begin{array}{l}24.5 \\
45.8 \\
29.6\end{array}$ & $<.01$ \\
\hline $\begin{array}{l}\text { Age }(\%) \\
16-30 \\
31-38 \\
39-48 \\
49-82\end{array}$ & $\begin{array}{l}31.7 \\
19.7 \\
24.1 \\
24.5\end{array}$ & $\begin{array}{l}32.9 \\
27.6 \\
22.9 \\
16.6\end{array}$ & $\begin{array}{l}17.4 \\
27.5 \\
30.5 \\
24.6\end{array}$ & $\begin{array}{l}13.7 \\
25.1 \\
34.1 \\
27.1\end{array}$ & $<.001$ \\
\hline $\begin{array}{l}\text { Quit at- } \\
\text { tempt }(\%) \\
\text { yes } \\
\text { no }\end{array}$ & $\begin{array}{l}19.7 \\
80.3\end{array}$ & $\begin{array}{l}40.9 \\
59.1\end{array}$ & $\begin{array}{l}64.3 \\
35.7\end{array}$ & $\begin{array}{l}71.9 \\
28.1\end{array}$ & $<.001$ \\
\hline Years smoked & 22.7 & 22.1 & 22.2 & 22.1 & ns \\
\hline $\begin{array}{l}\text { Cigarettes a } \\
\text { day }\end{array}$ & 20.7 & 20.4 & 20.1 & 19.8 & ns \\
\hline FTND score & 4.8 & 4.7 & 4.6 & 4.5 & ns \\
\hline
\end{tabular}

\section{Principal component analyses}

Separate principal component analyses were performed on the scores on the items of the pros of quitting, cons of quitting and perceived self-efficacy. The number of components was determined by: 1) the interpretability of the components; 2) the extent to which the items in a factor loaded uniquely on that factor; 3) the explained variance of the factors.

Within the items assessing the pros and the cons of quitting at least three components were expected: physical outcomes, social outcomes and selfevaluative outcomes. One item, assessing the positive monetary outcomes of quitting, was removed because it did not load uniquely on any component. In comparing the four smoker groups, this item was separately subjected to the same statistical procedures as the factors. After deleting this item the principal 
component analysis on the remaining fourteen pros of quitting items revealed four clearly interpretable factors (Table 3.3): long-term health consequences (3 items, $\alpha=87$ ); short-term health consequences (3 items, $\alpha=70$ ); social consequences ( 4 items, $\alpha=76$ ); self-evaluative consequences ( 4 items, $\alpha=.75$ ). The four components accounted for $65 \%$ of the total variance. With regard to the cons of quitting, the one factor solution fitted the best ( 6 items; $\alpha=58$ ). At least two components were expected with regard to self-efficacy: selfefficacy with regard to social situations and self-efficacy with regard to emotional situations. The analysis revealed the two expected factors (Table 3.3): social situations ( 4 items, $\alpha=81$ ) and emotional situation ( 3 items, $\alpha=86$ ). The two components accounted for $71 \%$ of the total variance.

\section{Cognitive determinants}

Table 3.4 shows the standardized $T$-scores of factors within the pros of quitting, the cons of quitting and the two factors and overall items assessing self-efficacy. The overall MANOVAs, indicated that all variables except the cons of quitting differed significantly $(p<.05)$ over the four groups. Only with regard to the short-term health consequences a significant interaction with age $(p<.05)$ was detected. The analyses were stratified according to the quartiles of age. Contrasts between the subsequent groups, using the MANOVA contrast procedure, are reported below.

Immotives scored significantly $(p<.05)$ lower than precontemplators on all factors within the pros of quitting, including the short-term health consequences in all four age groups, and on all four self-efficacy measures. On one self-efficacy overall item, "difficulty to stop myself smoking in all possible situations", however, the effect was in an unexpected direction: Immotives anticipated less difficulty stopping themselves smoking than precontemplators.

Precontemplators scored significantly lower than contemplators on longterm health, social and self-evaluative consequences, including on short-term health consequences in one age group ( $39-48$ years), and on three out of the four self-efficacy measures.

Contemplators scored significantly lower than preparers only on the perception of the self-evaluative consequences of quitting. 
Trable 3.3

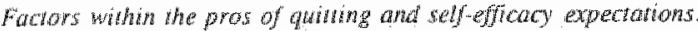

\begin{tabular}{|c|c|c|c|c|}
\hline \multicolumn{5}{|c|}{ Factors } \\
\hline diems pros of quiling & $\mathrm{Fl}$ & $F 2$ & $F_{3}$ & Fat \\
\hline My risk of lumg cancer will decline & 87 & & & \\
\hline My rist of $\mathrm{MD}$ will dechene & .83 & & & \\
\hline My rist of COPD mill declime & .77 & & & \\
\hline My hexalth will improve & & .76 & & \\
\hline I will look better & & 63 & & \\
\hline My stamina will increase & & .76 & & \\
\hline If will be lealliner for the people anound ne & & & .67 & \\
\hline I will calluse less inconvenience & & & .76 & \\
\hline I will be a beter example & & & .69 & \\
\hline The people around me will approve & & & 67 & \\
\hline 1 regred it when I huve whoked at dot & & & & .78 \\
\hline 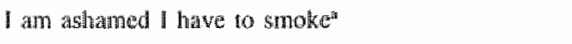 & & & & .74 \\
\hline I will be satisfied with myselt & & & & .63 \\
\hline \multirow[t]{2}{*}{ I will regret it if I get ilit } & & & & .64 \\
\hline & \multicolumn{2}{|c|}{ Factors } & & \\
\hline fenus selfefficacy & Fil & $\sqrt{2} 2$ & & \\
\hline When someone offers a cigarente & .86 & & & \\
\hline When I see someone enjoying smoking & .82 & & & \\
\hline When $\mathbb{I}$ an in a café or at a party & $.62^{*}$ & $(.43)$ & & \\
\hline When I feel uncomatortable when I am among others & $.59^{*}$ & $(.47)$ & & \\
\hline When I feel tense & & .84 & & \\
\hline Whan I foel atugery & & .87 & & \\
\hline When I foel depressed & & .80 & & \\
\hline
\end{tabular}

Note. All loadings $>40$ are depeted. "Because of difficulties with formalation the itern referred to a cansequence of not quinting. Pros: Fl=long-term healh consequerces; F2=shori-tem health consequences:

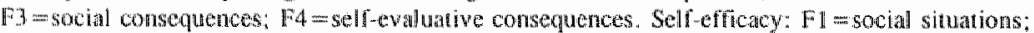

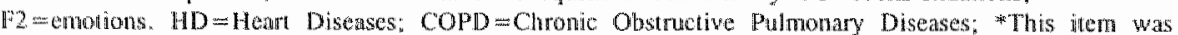
assigned to the factor on the basis of the interpreatbility. 


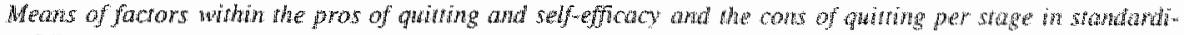
zed $T$ scores.

\begin{tabular}{|c|c|c|c|c|c|c|}
\hline \multirow[b]{2}{*}{ Variabile } & \multicolumn{4}{|c|}{ Stages of readiness } & \multirow[b]{2}{*}{$F$-teisto-value } & \multirow[b]{2}{*}{ Contrats } \\
\hline & Intr & $\mathrm{Pe}$ & c & $\operatorname{Pr}$ & & \\
\hline Pros of quituing & & & & & & \\
\hline Long-term health consequences & 46.5 & 50 & 51.9 & 52.2 & 30.47 .000 & $\mathrm{~m}<\mathrm{P}<<\mathrm{C}=\mathrm{Pr}$ \\
\hline \multicolumn{7}{|l|}{ Short-tierm health consequences } \\
\hline age $16-30$ & 49 & $\$ 1$ & 53.1 & 51.6 & 3.93 .009 & $\mathrm{lnt}<\mathrm{PC}=\mathrm{C}=\mathrm{Pr}$ \\
\hline $\operatorname{age} 31 \cdot 38$ & 45.5 & 50.9 & 532 & 52.8 & 13.3 .000 & $\|_{1 \mathrm{n}}<\mathrm{P}_{\mathrm{C}}=\mathrm{C}=\mathrm{P}_{\mathrm{r}}$ \\
\hline age $39-48$ & 45.3 & 49.1 & 53.1 & 53.9 & $17.21 \quad 000$ & $\ln <\mathrm{Pc}<\mathrm{C}=\mathrm{Pr}$ \\
\hline age $49-82$ & 42.6 & 48.7 & 51 & 53,3 & $22.14 \quad 000$ & $\operatorname{Im}<\mathrm{Pc}=\mathrm{C}=\mathrm{Pr}$ \\
\hline Soctal consequences & 45.7 & 50.3 & 52.2 & 53.8 & $59.3 \quad .000$ & $\operatorname{In}<\mathrm{P}_{\mathrm{C}}<\mathrm{C}=\mathrm{P}_{\mathrm{r}}$ \\
\hline Self-evaluative consequences & 42.6 & 50 & 54.4 & 56.3 & $219.9 \quad .000$ & $\operatorname{Im}<\mathrm{Pc}<\mathrm{C}<\mathrm{Pr}$ \\
\hline Monetary consequences & 48.5 & 49.9 & 50.6 & 50.9 & .002 & $\ln _{\mathrm{m}}<\mathrm{P}_{\mathrm{C}}=\mathrm{C}=\mathrm{P}_{\mathrm{T}}$ \\
\hline \multicolumn{7}{|l|}{ Cons of quiting } \\
\hline & 49.3 & 50.4 & 498 & 50.1 & $1.04 \quad .372$ & $\mathrm{I}_{\mathrm{H}}=\mathrm{PC}=\mathrm{C}=\mathrm{P}_{\mathrm{T}}$ \\
\hline \multicolumn{7}{|l|}{ Selfi-efficacy } \\
\hline Social situations & 46.8 & 49.1 & 52.6 & 53.7 & $22.38 \quad .000$ & Itn $<\mathrm{Pc}<\mathrm{C}=\mathrm{Pr}$ \\
\hline Emotions & 47.9 & .49 .2 & 52 & 52.9 & $40.85 \quad .000$ & $I_{n}<P_{C}<C=P_{1}$ \\
\hline Ability in all situations & 47.3 & 49.4 & 52.2 & 53.3 & $29.18 \quad .000$ & $\operatorname{Im}<\mathrm{Pc}<\mathrm{C}=\mathrm{Br}$ \\
\hline Difficulty in all situations & 51.5 & 49.7 & 49.8 & 49.4 & $3.88 \quad .009$ & Im $>P_{C}=C=P r$ \\
\hline
\end{tabular}

Note: All comparisons used standardized $\mathrm{T}$ scores $(\mathrm{Mean}=50)$ Im=immotives; $\mathrm{P}_{\mathrm{c}}=$ precontemplators; $C=$ contemplators; $\mathrm{Pr}=$ preparers. Im $<\mathrm{Pc}_{\mathrm{c}}$. Immotives score significantly lower $(\mathrm{p}<.05)$ than precontemplators.

\section{Predictive power of the stages}

Table 3.5 shows the scores with regard to the three measures of quitting behavior as reported at $\mathrm{T} 2$ and $\mathrm{T} 3$. In general, the higher the readiness to quit, the higher the percentages of smokers who were actively trying to quit. Only at fourteen months follow-up, fewer contemplators had quit for seven days compared to precontemplators, although the difference was not significant. At the three months follow-up, immotives and precontemplators differed significantly on all three measures of quitting behavior. Precontemplators and contemplators differed significantly on present quit attempt and 24-hour quit attempt while contemplators and preparers differed significantly on all three measures. At the fourteen months follow-up, immotives and precontemplators differed significantly on all three measures of quitting behavior. Precontemplators and contemplators only differed significantly on 24-hour quit attempt while contemplators and preparers differed on seven-day quit and present quit attempt. 


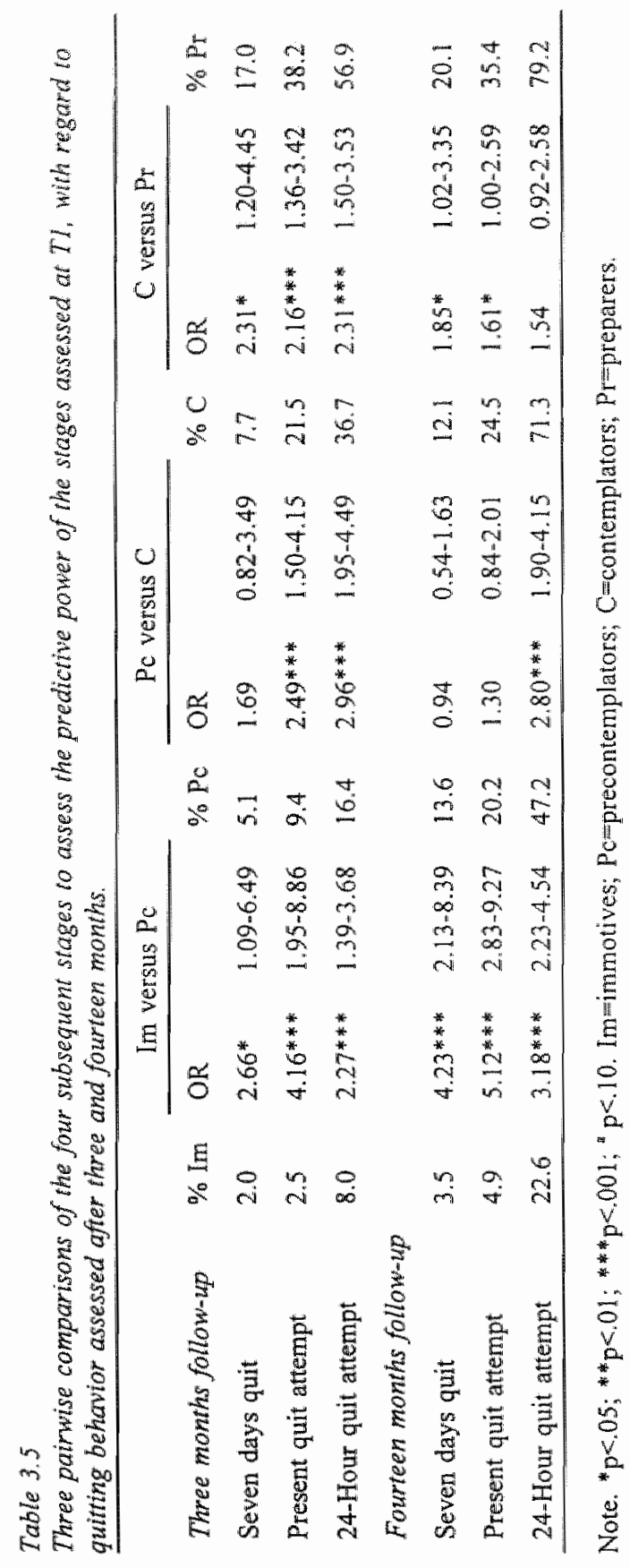




\section{Analyses including drop-onts}

Attrition from pre-test (Tl) to the post-test at 3 months was $28.8 \%(n=440)$ and at 1.4 months was $36 \%(n=555)$. Therefore, the analyses without the drop-outs might lead to biased results. As a check on such bias, an additional analysis was run in which dropouts were considered to have smoked for the last seven days, to not engage in a present quit attempt and to have made no 24-hour quit attempts since the last measurement. These conservative analyses revealed that mone of the results changed qualitatively. Only minor changes in odds ratios and $p$-values emerged.

\section{Discussion}

The stage model used in the present study was initially investigated by analyzing whether smokers in the various stages differed on cognitive measures. The results with regard to the cognitive differences supported the stage model, thereby replicating the findings of Dijkstra et al. (1997): smokers in the four stages differed on the central psychological determinants of readiness to change and behavior. Importantly, the stages did not differ on three measures of smoking behavior. Hence, the psychological differences seemed not just to reflect behavioral differences. Crittenden et al. (1994) reported similar results in their search for: "...movement within the early stages of change" (p. 498): within the group of smokers who were not planning to quit within the next six months, two groups were compared. Those who were and those who were not planning to quit. As in the present study, the groups differed on the perceived benefits of quitting and selfefficacy.

Besides identifying psychological differences between smokers in the four stages, the model was investigated by testing its predictive validity. The results with regard to the predictive validity also supported the stage model. In five of the six cases, the relation between readiness to change and the behavioral measure was linear: the higher the readiness to change, the higher the percentage of smokers who had quit. This is in line with the findings of DiClemente et al. (1991). Concluding, the self reported readiness to change was strongly predictive of quitting behavior. Especially the distinctions between immotives and precontemplators and between contemplators and preparers were predictive of quitting. Although precontemplators and contemplators differed on several cognitive measures, the distinction was the least predictive of point prevalence quitting. On the other hand, twice as many contemplators than precontemplators reported 24-hour quit attempts at three and at fourteen months follow-up. Furthermore, it might be concluded that the predictive power of the stages declined from three to fourteen months. This is in line with the concept of the stages referring to a measure of intention (Budd \& Rollnick, 1996; DiClemente et al., 1991): intention is the most proximate cognitive predictor of behavior but its power declines "... 
with the amount of time that intervenes between measurement of intention and observation of behavior" (Ajzen, 1988, p. 115).

At this point, a question which arises is what the surplus value is of the presently used stage definitions compared to a more traditional continuous measure of intention. The use of the present algorithms based on relevant cutoff points on a measure of readiness to change has some important benefits. First, smoker populations can be mapped using the stage algorithms (Mudde, Dolders \& De Vries, 1994; Velicer, Fava, Prochaska, Abrams, Emmons \& Pierce, 1995). Describing the smoker population in percentages of smokers in each stage may be more informative than using an average score on a continuous measure. Second, since the percentages of quitters in intervention studies largely depend on the readiness to change of the sample, there is little use in comparing success rates when no data on the readiness to change are available (also see DiClemente et al., 1991). Third, recognizing stages of behavioral change gives the opportunity to develop stage-matched interventions (Dijkstra, De Vries \& Roijackers, 1996; Prochaska, DiClemente, Velicer \& Rossi, 1993). Fourth, in contrast with a more continuous measure of intention, the present model allows us to describe specific psychological profiles of smokers in each stage.

The following typology of smokers in each stage could be made. Immothves have decided to keep on smoking or have vague long-term plans to quit somewhere in the future. As Crittenden et al. (1994) formulate it: they are not seriously thinking about quitting smoking. Smoking cessation is not an issue in their lives, they hardly see reasons for quitting and they have a biased view of quitting, apparent from the discrepancy between their judged ease of quitting and their (low) confidence in their ability to quit. Precontemplators see some reasons for quitting, have higher confidence that they are able to quit and, hence, have more concrete plans to quit. However, they are still poorly motivated to search for information on smoking and smoking cessation, as they are not planning to quit within the next six months. Contemplators see more reasons for quitting than precontemplator. They are specifically more aware of the long-term health, social and self-evaluative consequences of quitting. Furthermore, they think they are able to quit and, hence, they are planning to quit within the next six months. In contrast with preparers, they have not yet made the decision to actually quit in the short term, although they are open to information on smoking and smoking cessation (Prochaska et al., 1992).

In the present study, preparers differed from contemplators on only the self-evaluative consequences. This might mean that contemplators already anticipate all possible positive outcomes, and that preparers only have to process these outcomes more actively. This would lead to increased negative self-evaluation (Bandura, 1986). This self-evaluation might be the motive to actually plan a quit attempt in the short term: relief from the negative selfevaluation is anticipated. In contrast with the findings of DiClemente et al, (1991), preparers did not have higher self-efficacy than contemplators, in the 
present study. We assessed smokers in preparation only with the question: "are you planning to quit within one nonth?", whereas Diclemente and colleagues added the criterion "have been engaging in a quit attempt in the last twelwe months". However, post-hoc analyses adding the quit attempt criterion to the stage definitions revealed that boh groups of smokers still only differed on self-evaluation.

In conclusion, the stage model used in the current study was supported, although the significance of the proposed difference between precontemplators and contemplators needs further investigation. Furthermore, the present study may support the model with regard to smoking cessation, but there are no data, yet, with regard to these stages in other health behaviors. Many health behavior questionnaires assess stages of change and ask people whether they are planning to change within the next six months. Adding one question: "Are you planning to change within the next five years?", could yield data on the extent to which the presently proposed difference between immotives and precontemplators holds for other health behaviors. 


\title{
Chapter 4
}

\section{Self-evaluation and motivation to change: Social cognitive constructs in smoking cessation ${ }^{1}$}

\begin{abstract}
Bandura's Social Cognitive Theory (SCT) recognizes physical, social and self-evaluative outcome expectations. Especially the latter have a central place in motivation. Furthermore, SCT recognizes self-evaluation inhibiting processes. The relations among these cognitive factors and their relation to intention and behavior are summarized in the Self-evaluation Motivation Model and tested in the present study.

Smokers $(n=1546)$ were recruited by newspaper advertisements to join in a study on smoking cessation. They filled in a questionnaire assessing specific cognitive variables described in SCT. Follow-up assessments of quitting behavior were conducted after three and after fourteen months.

Multivariate regression analyses, first, indicated that the influence of physical and social outcome expectations and of dissonance reducing cognitions and attentional change processes on intention and behavior, was mediated by self-evaluation. Second, perceived long-term health outcomes were not related to self-evaluation when smokers used few attentional change processes. Third, the influence of dissonance reducing cognitions and attentional change processes on self-evaluation was partly mediated by their influence on physical and social outcome expectations. Suggestions for interventions to increase motivation to quit smoking are given.
\end{abstract}

This chapter is submitied for publication as: Dijkstra, A., De Vries, H., Kok, G., \& Foigackers, J. (1997). Selfevalnation and wotmation ho change: Social cogmine comstructs in swoking cessarion. Mantscript subminted for publication. Maastricht University. 


\section{Introduction}

In the Netherlands $70 \%$ of the smokers are not planning to quit within the next six months (Mudde, Dolders \& De Vries, 1994). In spite of ongoing national anti-smoking campaigns, these smokers persist in their habit and have no motivation to change their behavior. From a theoretical point of view, the low motivation to quit might be understood in terms of a cognitive state which preserves itself. In the present study, aimed at understanding the role of cognitive factors in low motivation to quit, the Social Cognitive Theory (SCT) of Bandura (1986) was used as the theoretical framework.

In SCT people regulate their behavior on the basis of anticipated effects of their behavior. This anticipation is referred to as outcome expectations which can be viewed as cognitive representations of future events. Bandura (1997) distinguishes three kinds of outcome expectations: physical, social and selfevaluative outcome expectations. Physical and social outcome expectations refer to the anticipation of personal experience of extewnal events, such as pain and social reward, respectively. Self-evaluative outcome expectations, however, refer to the anticipation of aversive or rewarding experiences caused by (self set) intemal standards, such as being dissatisfied or proud. Present negative self-evaluation is considered to be accompanied by the anticipation of relief of this aversive state in the case the cause of the negative selfevaluation is removed. This anticipation is a self-evaluative outcome expectation. Self-evaluation originates from the comparison of accomplishments with personal standards or goals. People will react with negative or positive selfevaluation depending on whether the demands of the standards or goals are met (Bandura, 1986; also see Keltner \& Buswell, 1996). Personal standards, or the goals based on these standards, develop through modeling and they represent a person's values. The construct of self-evaluation seems related to psychological constructs such as self-approval or disapproval (Janis \& Mann, 1977), moral norm (Boyd \& Wandersman, 1991; Manstead \& Parker, 1995), and anticipated regret (Richard, Van der Pligt \& De Vries, 1995). The latter two constructs are recently incorporated in the Theory of Planned Behavior (Ajzen, 1991; Godin \& Kok, 1996; Manstead \& Parker, 1995).

As with physical and social outcome expectations, people engage in behavior of which they think it will lead to positive self-evaluation, and avoid behavior of which they think it will lead to negative self-evaluation. In that, physical, social and self-evaluative outcome expectations can be regarded as exerting their power on behavior in a parallel fashion. However, the three sorts of outcomes also seem to work serial. Bandura (1986): "Extemal influence [external outcome] will have its greatest impact on behavior when it is compatible with personal standards. Under these conditions, rewardable acts are a source of self-satisfaction and punishable ones are self-censured" (p. 375). For example, the experience of short-term health benefits in smoking cessation might lead to positive self-evaluation which, in turn, has its impact on behavior. In other words, the power of social and physical outcome 
expectations might at least partly be mediated by self-evaluation.

The important role of expected selfevaluative outcomes in motivation, is supported in several studies (Bandura \& Cervone, 1983; Gibbons, 1990; Prussia \& Kinicki, 1996). Wuth regard to smoking cessation, Dijkstra, Bakker and De Vries (1997) found that (using a scale measuring physical, social and selfevaluative outcomes), self-evaluative outcome expectations discriminated between smokers with low motivation and other smokers. In another study on motivation in smoking cessation, Dijkstra, Roijackers and De Viries (in pressc), found that within the scale measuring positive outcome expectations, the self-evaluative outcomes were the only outcomes discriminating between smokers who were, and smokers who were not planning to quit within one month. These findings suggest that the stronger people evaluate themselves negatively because of engaging in smoking behavior, the more they are motivated to change that behavior because they anticipate relief from the aversive self-evaluative experience.

Besides the differentiation of physical, social and self-evaluative outcome expectations, positive and negative outcome expectations of behavior or behavior change can be distinguished. In general, positive outcome expectations with regard to behavior change (expected reward or removal of punishment) are considered to facilitate behavior change, whereas negative outcome expectations with regard to behavior change (expected impending punishment or removal of reward) are considered to inhibit behavior change. Some studies suggest that positive and negative outcome expectations, or pros and cons of changing the behavior, have differential impact on behavior (Dijkstra, De Vries \& Bakker, 1996; Velicer, DiClemente, Prochaska \& Brandenburg, 1985): in motivating people to change their behavior the anticipated gain of changing may be more powerful than the anticipated loss of changing (Dijkstra et al., 1996; Prochaska, 1994).

\section{Self-evaluation regulation}

Next to the influence of accomplishments and the resulting external events on self-evaluative reactions, Bandura (1986) recognizes means by which selfevaluative reactions can be inhibited. First, people try to keep up their positive self-regard by reinterpreting and restructuring the perception of, for example, threatening events. This cognitive means seems to refer to phenomena described as 'dissonance teduction' (Festinger, 1957), and the use of "permission giving beliefs" (Beck. Wright, Newman \& Liese, 1993). Chapman, Wong and Smith (1993) measured dissonance reducing cognitions, or excuses 10 smoke, in smokers and ex-smokers. Ex-smokers used nine out of the fourteen beliefs to a lesser degree than smokers. Thus, at the moment that the external threat is no longer present, i.e. the individual has quit smoking, the reinterpreting and restructuring perceptions are no longer needed.

Second, people try to keep up their positive self-regard by avoiding information through selective inattention. This cognitive means seems related 
to Janis \& Mann's (1977) construct of 'defensive avoidance", which is described in terms of selective attention and avoidance of thinking about information, and to the concept of the 'experiential processes of change' (Prochaska, 1984; Prochaska, Velicer, DiClemente, \& Fava, 1988). Prochaska, DiClemente and Norcross (1992) distinguish between five experientiall processes of change which are covert activities that people use when they change their behavior. These processes are measured in terms of frequency of thinking, recalling, considering, noticing or experiencing information on smoking and smoking cessation. The construct typically is operationalized as the frequency in which the topic of smoking or smoking cessation is in the focus of the attention of an individual (Prochaska et al., 1988). This operationalization seems supported by Janis \& Mann (1977) as they state:"...the person spends little time thinking about...."(p. 74).

The importance of identifying self-evaluation influencing mechanisms, lies in the possibilities to intervene on these mechanisms in order to increase selfevaluation and, hence, increase motivation and to stimulate behavior change.

The Self-Evaluation Motivation model (Figure 4.1), based on the SCT, summarizes the cognitive factors and their global relation and may serve a heuristic function. Although in the Social Cognitive Theory self-efficacy expectations are considered to be important in motivation and intention to change behavior, the inclusion of this variable would be beyond the scope of the present article.

The first goal of the present study was to assess whether the influence of positive and negative outcome expectations on intention and behavior is mediated by self-evaluation. It was expected that outcome expectations have an independent effect on intention and behavior (parallel exertion). In addition, it was expected that a part of the effect of outcome expectations is mediated by self-evaluation (serial exertion).

The second goal of the present study was to assess how positive and negative outcome expectations (with regard to external events) and self-evaluation inhibiting processes influence self-evaluation. Two possible relations were hypothesized. First, it might be that their is an interaction between extemal outcomes and self-evaluation inhibiting processes on self-evaluation. In other words, only when people use few dissonance reducing cognitions or many attentional change processes, the perception of external outcomes influences self-evaluation. Second, the effect of self-evaluation inhibiting processes on self-evaluation might be mediated by perceived external outcomes. In other words, it might be that dissonance reducing cognitions infuence self-evaluation by distorting the meaning of the external outcomes and that attentional change processes influence self-evaluation by lowering the salience of the outcomes. 


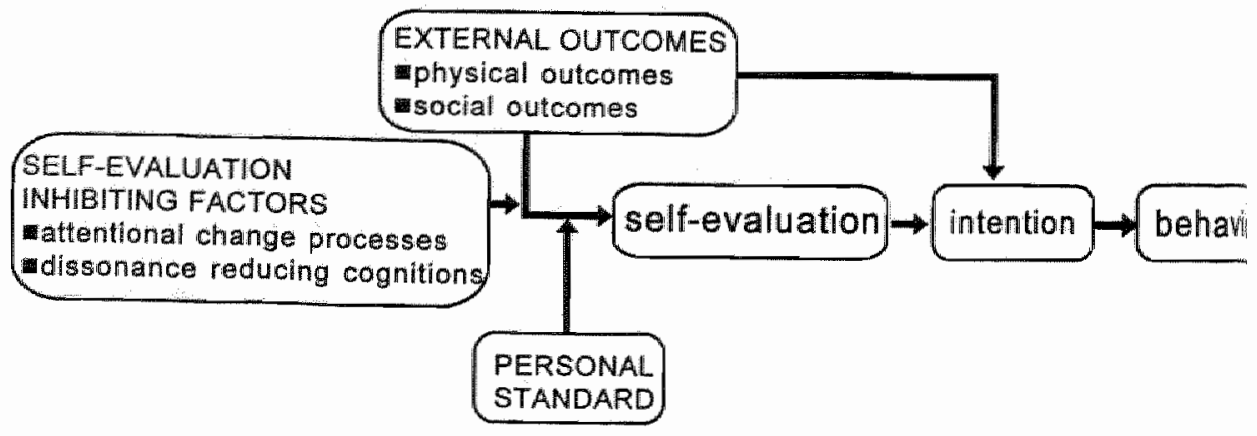

Figure 4.1 The Self-Evaluation Motrvation model based on the Social Cognitive Theory (Bandura, 1986).

\section{Method}

\section{Participant recruitment and design}

Smokers were recruited by advertisements in local newspapers throughout the Netherlands. They were asked to volunteer for a research project on minimal interventions for smoking cessation including the possibility of being a control group member. Participants completing all three questionnaires were offered the opportunity for 10 bonus prizes amounting $100 \$$. After participants had phoned to the university in order to register $(n=1733)$, they were sent the pre-test questionnaire which could be returned with a pre-paid envelope. After two weeks 1546 pre-test questionnaires $(89.2 \%)$ had been returned (sixty questionnaires of smokers of pipe or cigar and questionnaires filled out incompletely were excluded). Three sets of data were used in the present article: the first, cross-sectional, set concerns the pre-test data (T1; $\mathrm{N}=1546$ ), while both longitudinal sets concern a subsample of this population $(\mathrm{N}=385$ at $\mathrm{T} 1)$ who received additional measurements after three months ( $\mathrm{T} 2$; $\mathrm{N}=251$ ) and after fourteen months ( $\mathrm{T} 3 ; \mathrm{N}=230$ ).

\section{Questionnaire}

Positive and negative outcome expectations. The items in the scales assessing the positive and negative outcome expectations were validated in an earlier study (Dijkstra et al., 1996). The items could be scored from "not sure" or "not expecting a certain outcome" $(0)$ to a "strong expectation of the 
outcome" (3) and most items were in the following format: "If I quit smoking, then...".

The six items in the scale assessing the expected negative outcomes referred to the anticipated negative consequences of behavion change (Cronbach's alpha of 57 ; If I quit smoking, then ...: I will gain weight; it will be harder to socialize; I will miss a means to relax; I will miss the smoker image; I will be bored more often; I will experience withdrawal symptoms). Within the scale assessing the negative outcomes (NegOut), the one factor solution fitted the best.

The fifteen items in the scale assessing the expected positive outcomes referred to the positive consequences of behavior change $(\alpha=87)$. Principal component analyses with varimax rotation revealed four clearly interpretable factors within the scale measuring positive outcome expectations (Dijkstra et al., in press-c). Two components referred to the physical outcomes of quitting: long-term health outcomes (LongOut; 3 items, $\alpha=87$ ) and the shortterm health outcomes (ShortOut; 3 items, $\alpha=.70$ ). The items in the scale measuring long-term health consequences addressed the decreased risk on lung cancer, coronary heart disease (CHD) and chronic obstructive pulmonary disease (COPD). The items in the scale measuring the short-term health consequences of quitting addressed improved health, better stamina and looking better. One component referred to the social consequences of quitting: (SocOut; 4 items, $\alpha=76$ ). The items in this scale addressed approval from the social environment, better health and less inconvenience for people in the social environment and being a better example. One component referred to the self-evaluative consequences of quitting: (SelfOut; 4 items, $\alpha=.75$ ). Two items assessed present negative self-evaluation ("I regret it when I have smoked a lot" and "I am ashamed I have to smoke"). The higher the score, the greater the reward or removal of punishment in the case of quitting. One item referred to the reward of quitting ("If I quit smoking I will be satisfied with myself") and another item referred to the punishment caused by not quitting ("I regret it if I get ill").

Dissonance reducing cognitions. The items of the scale measuring dissonance reducing cognitions (DisCog) were derived from a larger pool of items measured in a pilot study among 101 medical students. This resulted in an eight-item scale. The items could be scored from "I do not agree" (-2) to "I do agree" $(+2)(\alpha=72)$ and were formulated to finish the sentence: "Smoking can make me ill, but....". The items gave reasons (or excuses) why it is alright to smoke, despite the well-known detrimental effects. A typical excuse may be true in itself but only addressing half the truth (for example: "I know heavy smokers who live a long and healihy life" or "I am exposed to so many risks in my life") or it may actually have little ground in reality (for example: "If smoking would be really that bad, it would be prohibited").

Attentional change processes. The items of the scale measuring attentional change processes (AttProc) were based on a questionnaire developed by Prochaska et al. (1988) which was translated into Dutch. Prochaska et al. 
distinguish between five experiential processes of change. Whereas according to Prochaska et al. the construct is transtheoretical, in the present study, attentional change processes were considered to refer to concrete and measurable allocation of attention. Attentional change processes are considered to work as an information filter which regulates, on the basis of a need to process or avoid information, the frequency and time with which certain information is in the conscious focus of the attention. As such, these change processes are considered to be a cause of the processing of domain-specific information (i.e. information on smoking and smoking cessation). That is why two experiential processes, self-reevaluation and dramatic relief, were not included in the present change process measure: self-reevaluation and dramatic relief seemed to assess an effect of the processing of domain-specific information, i.e. feeling disappointed or being upset, rather than a cause of this processing.

Thus, three processes were considered which may influence self-evaluation by means of attentional focus on specific (smoking related) information: Consciousness raising (for example: "I recall articles dealing with the problem of quitting smoking") environmental evaluation (for example: "I consider the view that smoking can be harmful to the environment") and social liberation (for example: "I notice that public places have sections set aside for smoking"). Each process was measured with two items which summed to one attentional change process scale $(\alpha=, 78)$. The six items were formulated in terms of thinking, noticing or recalling information or cues with regard to smoking and could be scored in terms of frequencies from "never" (0) to "very often"(4).

Intention to quit and quitting. Intention to quit was measured with a composite of two 10-point scales: "Do you intend to quit smoking?" ("not at all" (1) to "very much" (10)), and "How probable is it that you will quit smoking?" ("not probable at all" (1) to "very probable" (10)). The intention score was the average item score. Quitting behavior was measured by asking participants whether they were engaging in a quit attempt at the present time of the measurement (yes/no).

Smoking behavior. Heaviness of smoking was measured by the Fagerström Test for Nicotine Dependence (Heatherton, Kozlowski, Frecker \& Fagerström, 1991) which assesses smoking habits: How much do you smoke? How soon after awaking? Is it hard to smoke not in public places? Do you smoke when you are ill? Do you smoke more in the morning and which cigarette is most difficult to give up? The minimum possible score was 0 , the maximum $10(\alpha=71)$. Furthermore, smoking behavior was measured by asking smokers how many years they had been smoking, how many cigarettes they smoked on the average and whether they had been engaging in a 24 hour quit attempt in the last 12 months.

Demographics measured were gender, age and education level which was categorized as low, medium or high level of education.

No biochemical verification of the self report quitting behavior was 
conducted, first, because it was expected that the announcement of a biochemical verification would increase non-responding and drop-out. Second, the present study was considered to be one of low demand. Hence, selfreports on smoking were considered to be valid (see Velicer, Prochaskin, Rossi \& Snow, 1992).

\section{Analyses}

In order to check whether and how the cognitive measures related to each other, Pearson correlations between the seven neasures and intention were computed.

The first main question to be answered was whether the influence of physical and social positive outcome expectations, negative outcome expectations and self-evaluation inhibiting processes on intention and behavior is mediated by self-evaluative outcome expectations. In the first step, the six cognitive factors mentioned above (LongOut, ShortOut, Socout, NegOut, DisCog, AttProc, excluding SelfOut), were entered in a regression analysis to predict intention to quit. Second, to test whether and to what extent the six cognitive factors exert their impact via self-evaluation, the self-evaluative outcome (SelfOut) was entered the same equation to predict intention. "Third, this model was tested longitudinally in predicting quit attempts at $T 2$, and $T 3$ with a logistic regression.

The second main question concemed an interaction between self-evaluation inhibiting processes on the one hand (DisCog, AttProc) and four factors of extermal outcome expectations (LongOut, ShortOut, SocOut, NegOut) on the other hand, in predicting self-evaluative outcomes (SelfOut). The resulting eight interactions and the six main factors were entered in the regression analysis. Non-significant interactions ( $p>05$ ) were removed from the analysis. Whenever an interaction was significant, the subsequent analyses were stratified according to tertiles of the attentional change processes or dissonance reducing cognitions. When no interaction would occur, the alternative hypothesis was tested. To test whether self-evaluation inhibiting processes (DisCog, AttProc) exert their influence on self-evaluative outcomes via the external outcomes, first, they were entered in a regression to predict selfevaluative outcomes. Second, the external outcomes (LongOut, Shortout, SocOut, NegOut) were added to the equation to test whether self-evaluation inhibiting processes still had a main effect on self-evaluative outcomes.

\section{Participant characteristics}

Of the 1540 respondents, $59 \%$ was female, the average age was 39.7 and $22 \%$ was classified as low educated, $43 \%$ as medium educated and $35 \%$ as high educated. The respondents smoked an average of 20.3 cigarettes a day and they had smoked for an average of 21.8 years. The average FTND-score (the scale ranging from $0-10$ ) was 4.6 . Of the respondents, $44.9 \%$ had 
engaged in a serous quit attempt in the last twelve months. Finally, the mean imtention to quit (the scale ranging from 1 - 10) was 5.9 .

\section{Results}

\section{The cognitive measures}

Table 4.1 shows the Pearson correlations between the seven cognitive measures and intention. The main results can be summarized as follows: correlations between the four factors of positive outcome expectations range from 41 to .57 , meaning that they are related but referring to different sorts of outcomes. The correlations between the four factors of positive outcome expectations and the negative outcome expectations were significant but small $(\leq 2)$, thereby supporting the distinction between positive and negative outcomes. Dissonance reducing cognitions had significant negative relations with all (except one) variables. Thus, as expected, the more excuses smokers use, the fewer positive outcomes they anticipate and the fewer attentional change processes they use. Attentional change processes had a positive relation with all outcome factors. Thus, as expected, the higher the frequency of domain specific information in the focus of the attention, the more positive outcomes of quitting smokers perceive. Finally, intention had positive correlations with positive outcomes, of which the correlation with selfevaluative outcomes was the highest. Intention had no relation with the negative outcomes and a negative relation with dissonance reducing cognitions. Concluding, the relations are in the expected directions.

Table 4.1

Pearson correlations between eight cagnitwe measures.

\begin{tabular}{|c|c|c|c|c|c|c|c|c|}
\hline & $\begin{array}{l}\Downarrow \text {. } \\
\text { Selfout }\end{array}$ & $\begin{array}{l}2 . \\
\text { LongOut }\end{array}$ & $\begin{array}{l}3 . \\
\text { Shoriout }\end{array}$ & $\begin{array}{l}4 . \\
\text { SooOut }\end{array}$ & $\begin{array}{l}5 . \\
\text { NegOut }\end{array}$ & $\begin{array}{l}6 . \\
\text { AttProc }\end{array}$ & $\begin{array}{l}7 . \\
\text { Discog }\end{array}$ & $\begin{array}{l}8 . \\
\text { Intention }\end{array}$ \\
\hline 1. Sellout & 1.00 & & & & & & & \\
\hline 2. LongOut & .42 & 1.00 & & & & & & \\
\hline 3. Shortout & .48 & .53 & 1.00 & & & & & \\
\hline 4. Soo Ont & .57 & .43 & $4 H$ & 1.00 & & & & \\
\hline 5. NegOut & .19 & .14 & 16 & .20 & 1.00 & & & \\
\hline 6. AltProc & .47 & .28 & .28 & .48 & .18 & 1.00 & & \\
\hline 7. Discog & -.36 & -.36 & -.31 & -27 & $-.04^{*}$ & -.17 & 1.00 & \\
\hline 8. Intention & .57 & .27 & 32 & .37 & $.00^{n}$ & .30 &. .25 & 1.00 \\
\hline
\end{tabular}

Note: all correlations, except two (" mon-significant correlation), were significant ( $<<01$ ) 


\section{Self-evaluation as mediator}

Table 4.2, firstly, shows the explained variance and betas of the variables resulting from a stepwise regression analyses in which the cognitive factors (SelfOut excluded) predicted intention. The most important variable explaining the variance of intention was social outcome, explaining $14 \%$ of the variance. Short-term health outcome added 3.2\%. After that, dissonance reducing cognitions, attentional change processes and negative outcome added litile, but significantly to the explained variance. The only variable not entering the equation $(p>4)$ was the long-term health outcome.

\section{Table 4.2}

Cross-sectional prediction of intention by cognitive factors, without and with self-evaluative outcome expectations as predictor, using stepwise regression analyses.

\begin{tabular}{|c|c|c|}
\hline Without SelfOut & $\mathrm{R}^{2}(\%)$ & B standardized \\
\hline SocOut & $14.0^{* * *}$ & $0.22^{* * *}$ \\
\hline ShortOut & $17.2^{* * * *}$ & $0.17^{* * *}$ \\
\hline AttProc & $18.7^{* * *}$ & $0.15^{* * *}$ \\
\hline DisCog & $20.0^{* * * *}$ & $-0.12^{* * *}$ \\
\hline NegOut & $21.0^{* * * *}$ & $-0.10^{4 * *}$ \\
\hline With Selfout & $\mathrm{R}^{2}(\%)$ & B standardized \\
\hline SelfOut & $32.3^{* 4 *}$ & $0.52^{* * *}$ \\
\hline NegOut & $33.5^{\mathrm{sm} *}$ & $-0.13^{* * *}$ \\
\hline SocOut & $34.0^{* * *}$ & $0.07^{* * 4}$ \\
\hline ShortOut & $34.2^{* *}$ & $0.06^{\circ}$ \\
\hline
\end{tabular}

Note. "p<.05; "p<.01; " $p<.001$ : in the $\mathrm{R}^{2}$ column the significancies refer to the F-change test, in the $\mathbb{B}$ column to the t-test of $B$.

Standardized Bs are those from the final equation.

Table 4.2, secondly, shows the explained variance and betas when the variable self-evaluative outcome was entered in the equation. Self-evaluative outcome explained $32.3 \%$ of the variance. After that, negative outcome added little $(1.2 \%)$ but significantly. As in the former equation, the beta of negative outcome was negative, meaning that the more negative outcomes of quitting were perceived, the lower the intention to quit. Social outcome and short-term health outcome significantly explained respectively about $0.5 \%$ and $0.2 \%$. Thus, the social, physical and negative outcomes had little independent impact on intention to quit. 
Next, the model was tested longitudinally. The pre-test scores on the same cognitive factors as in the cross-sectional regression analyses, including selfevaluative outcome, were entered in two separate logistic regression analyses to predict the engagement in a quit attempt at three months and at fourteen months follow-up. The results showed that in predicting behavior with the same model, self-evaluative outcome was the only significant predictor at both follow-ups $\left(O R^{2}=2.2 ; \quad \mathrm{p}<.001 ; \quad O R=1.17 ; \mathrm{P}<.05\right)$. The data seem to support the notion that self-evaluative outcome expectations mediate the influence of the other cognitive factors on intention and behavior.

\section{A conditional effect of outcome expectations on self-evaluation}

First, it was hypothesized that expected external outcomes lead to selfevaluation, only when this information about the external events is actively processed (i.e. few dissonance reducing cognitions or many attentional change processes). Therefore, interactions of social, short-term health, long-term health and negative outcome on the one hand with the self-evaluation inhibiting processes on the other hand, were also entered in the analysis to predict self-evaluation. In a stepwise regression analysis only the interaction of longterm health outcome and attentional change processes was significant $(p<.001)$.

In order to search for the meaning of the interaction, the data were stratified according to tertiles of scores on the attentional change processes. In Table 4.3 the results from the stepwise regression analyses to predict selfevaluative outcomes from all six cognitive variables are depicted for the smokers scoring low, medium and high on the attentional change processes. The interaction between long-term health outcome and attentional change processes was explained by the following finding: whereas in the medium and highest vertile of attentional change processes the long-term health outcome added significant (but little) to the explained variance of self-evaluative outcomes, in the lowest tertile the long-term health outcome had no relation with selfuevaluative outcomes.

Because the interaction hypothesis was only partly verified, next, it was hypothesized that dissonance reducing cognitions and attentional change processes exert their influence on self-evaluative outcomes via social, shortterm health, long-tern thealth and negative outcomes. First, to check to what extent dissonance reducung cognitions and attentional change processes within the tertiles explained variance in self-evaluative outcome, only both variables (DisCog, AttProc) were entered in a regression analysis. In the lowest tertile, dissonance reducing cognition and attentional change processes respectively explained $6.0 \%$ and $3.0 \%$ of the variance, in the medium tertile $1.0 .4 \%$ and $2.2 \%$, and in the highest tertile $13.7 \%$ and $7.9 \%$. Second, social, short-term health, long-term health and negative outcomes were entered in the analysis.

$\mathrm{OR}=\mathrm{odds}$ ratio 
Once the outcome factors were entered in the equation, dissonance reducing cognitions and attentional change processes added less to the explained variance of self-evaluative outcomes: in the lowest tertile $1.3 \%$ and $0 \%$ respecively, in the medium tertile $2.4 \%$ and $0.4 \%$ and in the highest tertile $4.8 \%$ and $3.0 \%$. Thus, it seems that the influence of dissonance reducing cognitions and attentional change processes on self-evaluative outcome is partly mediated by their influence on the outcome factors.

Table 43

The relation of the cognitive factors on self-evaluative outconnes" per tertile of the attentional change processes, using stepwise regression analyses.

\begin{tabular}{|c|c|c|}
\hline Lowest tertile AttProc & $\mathrm{R}^{2}(\%)$ & standardized B \\
\hline Socout & $22.7^{* * *}$ & $0.33^{* * 2}$ \\
\hline Shortout & $28.7^{* * *}$ & $0.24^{* * *}$ \\
\hline NegOut & $29.9^{* *}$ & $0.13^{*+}$ \\
\hline DisCog & $31.2^{* *}$ & $-0.13^{* *}$ \\
\hline \multicolumn{3}{|l|}{ Medium tertile AttProc } \\
\hline SocOut & $23.6^{* * *}$ & $0.35^{* * *}$ \\
\hline ShortOut & $30.7^{* * *}$ & $0.20^{* * *}$ \\
\hline DisCog & $33.1^{* * *}$ & $-0.15^{* * *}$ \\
\hline LongOut & $33.7^{* *}$ & $0.09^{\circ}$ \\
\hline AttProc & $34.1^{*}$ & $0.07^{\circ}$ \\
\hline \multicolumn{3}{|l|}{ Highest tertile AttProc } \\
\hline Shortout & $20.5^{* * *}$ & $0.23^{* * *}$ \\
\hline SocOut & $30.1^{* *}$ & $0.27^{* * *}$ \\
\hline DisCog & $34.9^{* *}$ & $-0.22^{* *}$ \\
\hline AttProc & $37.9^{* * *}$ & $0.19 * *$ \\
\hline LongOut & $38.3^{*}$ & $0.09^{\circ}$ \\
\hline
\end{tabular}

Note. $p<.05 ; "$ " $p<.01 ; "$ " $p<.001$ : in the $\mathrm{R}^{2}$ column the significancies refer to the F-change test, in the $B$ column to the t-test of $B$. " The following cognitive factors were entered: SelfOut, LongOut, ShortOut, SocOut, NegOut, AttProc, Discog. 


\section{Discussion}

The first major finding was that the influence of external positive outcome expectations (physical and social outcomes) and self-evaluation inhibiting processes on intention and behavior, indeed seemed mediated by selfevaluative outcome expectations. The longitudinal test, using the same model, verified the central importance of self-evaluative outcome expectations in smoking cessation. It might be concluded that, in smoking cessation, positive extemal outcomes and self-evaluative outcome expectations work serial for a large part. In contrast, negative outcome expectations were hardly predictive of intention and quitting behavior and were not mediated by self-evaluative outcome expectations.

The second finding was that the influence of the long-term expected outcomes on self-evaluative outcome expectations was conditional on a certain level of attentional change process use. In the present sample of 1540 smokers this would mean that in almost 500 smokers (lowest tertile), the anticipation of the long-term health consequences of smoking have no motivating power. This might implicate that their is little use in trying to motjvate these smokers with information on long-term health outcomes: A score of 7 or lower on the presently used attentional change process measure indicates that the information processing 'door' was closed for information on the long-term health consequences.

The third finding was that self-evaluation inhibiting processes seem to exert their influence on self-evaluative outcome expectations via their effect on the external outcomes (physical and social outcomes). It might be that dissonance reducing cognitions distort the meaning of the external outcomes and attentional change processes infuence the salience of the external outcomes. Besides their influence on external outcomes, self-evaluation inhibiting processes still had an independent effect on self-evaluative outcome expectations. This remaining effect might be due to measurement error. It might vanish when, for example, extra social outcome factors (Dijkstra, Bakker \& De Vries, 1997) are added taking over explained variance in selfevaluative outcome expectations.

In conclusion, low motivation to quit may be caused by several cognitive fictors which all seem related to self-evaluative outcome expectations. In principle, an intervention would have to increase the score on the presently used measure of self-evaluative outcome. In the present study, self-evaluative outcome was measured by asking whether smokers were ashamed or felt regret at present, whether they would be satisfied in the case of quitting and whether they would regret it if they would get ill from smoking. The former two questions measure the present state of negative selfevaluation directly, whereas it may be argued that the answers on the latter two questions measure present negative self-evaluation indirectly. That is, high satisfaction in the case of quitting may reflect present dissatisfaction with regard to smoking and much anticipated regret may reflect the present state of aware- 
ness of discrepancy between what someone 'should' do (on the basis of the personal standard) and the present behavior. Hence, a smoking cessation intervention will have to increase the scores on the measure of self-evaluative outcome expectations by increasing negative self-evaluation.

With regard to the development of interventions to motivate smokers to quit, the following preliminary recommendations could be made. First, in order to increase negative self-evaluation, smokers must be offered the facts on the consequences of their smoking. Clearly, information on the short-term health consequences and the consequences of smoking for the social environment seemed the most important (also see McMaster \& Lee, 1991). On the basis of the present data it might be concluded that information on the longterm health consequences is not needed: long-term health outcomes were not predictive of intention and behavior. However, long-term health consequences had positive and significant univariate correlations with short-term health outcomes, social outcomes and intention. Thus, it seems that the anticipation of long-term health outcomes influences intention (in a multivariate model) via other cognitive factors. For example, it might be that facts on the longterm consequences make smokers more aware of the short-term physical consequences or the detrimental facts of smoking for people in their environment. Moreover, a smoking cessation intervention without any reference to the long-term consequences such as lung cancer or CHD seems counterintuitive.

The influence of negative outcomes on intention, behavior and selfevaluative outcome expectations was small. Hence, it is questionable whether in a smoking cessation intervention the impact of negative outcomes has to be lowered by challenging and restructuring these outcomes. It may be that directing and changing negative outcomes is more appropriate in a later (relapse prevention) phase (Dijkstra, De Vries \& Bakker, 1995; Marlatt \& Gordon, 1985).

Second, an intervention has to increase self-evaluation by decreasing the adherence to dissonance reducing cognitions. Beck et al. (1993) treat these cognitions, which they call 'permission giving beliefs', within the framework of cognitive therapy and propose challenging and restructuring as a means to lower the impact of these beliefs. However, no data are available on the efficacy of interventions specifically designed to challenge and restructure excuses to smoke. Thus, at present no lirm recommendations can be made to direct these beliefs in smoking cessation interventions.

Third, a smoking cessation intervention has to increase self-evaluation by increasing the attentional change processes. As attentional change processes are conceptualized as the lime or frequency with which information on smoking and smoking cessation is in the focus of the attention, we might confront smokers frequently with information on this topic. For example, computer-generated tailored interventions can be offered more than once and may be an appropriate means to communicate health information to smokers with low motivation to quit (Dijkstra, De Vries, Roijackers \& Van Breukelen, 
in press-d; Prochaska, DiClemente, Velicer \& Rossi, 1991). Furthermore, we would like to instate a gernume attentional change process. We assume that there must be a psychological motive to increase the scanning of smoking outcome related information and that interventions will have to direct this motive. At this point we need to know what the "natural" causes are of selfevaluation inhibiting processes. According to Janis and Mann (1977), people engage in 'vigilant searcl?' (increased attention for specific information) when they feel threatened but at the same time feel that they can manage the situation. In line with this, Maibach and Parrot (1995) recommend that interventions have to contain information to enhance the personal efficacy and response efficacy. In other words, interventions aimed at motivating smokers to quit would not only have to communicate on why smokers should quit (information on outcomes) but also on how they can quit, in order to instate genuine attentional change processes.

It might be argued that the more attentional change processes smokers use, the more they are aware of what they are doing, the more they experience the discrepancy between their personal standards and their actual behavior and the more they evaluate themselves negatively. Bandura (1986), indeed, considers self-observation as the primary condition to engage in self-evaluation (also see Gibbons, 1990). Hence, smoking cessation interventions would have to increase self-observation. Especially, computer-tailored interventions can provide smokers with several sorts of individual feedback that may increase the awareness of discrepancy. A tailored intervention might explicitly show individual smokers the discrepancy between their perception of what is desirable and what 'should be' on the one hand and the consequences of their behavior on the other hand. For example, smoking individuals might be confronted with their personal standards or goals, such as "being a reasonable person', or 'maintaining good health'. Although we assume that most people do have these standards, it is expected that people differ with respect to the content and the extent to which these standards do actually guide their behavior (Bandura, 1986; Pervin, 1989). Clearly, further research on the triggering of genuine attentional change processes and increasing awareness of discrepancy is needed.

The present study has its limitations. First, additional measures of cognitive constructs might be needed to understand the causes of the use of selfevaluation inhibiting processes. For example, constructs like risk perception and experienced threat might shed light on motives to avoid information on physical and social outcomes. Second, the operationalization of self-evaluation inhibiting processes can be criticized: the dissonance reducing cognitions only referred to the distortion of information on the physical threat of smoking. Furthermore, it may be that the use of the short versions of three experiential processes is not a complete measure of attentional change processes. Moreover, the retrospective self-report, in which smokers are asked to rate the frequency of thinking, noticing or recalling certain information, might be unreliable. This report might be influenced by selective memory, 
social desirable answers and environmental cues who instate thoughts on smoking and smoking cessation. Third, although the theory proposes specific causal relations, the present data can not be conclusive with regard to causality. Experimental studies are needed to test specific cansal relations.

The Self-Evaluation Motivation model as presented in Figure 4.1, may be a useful working model, although the relation between the variables seem more complex than depicted. Ideally, future research will test the causal relations as proposed in the model experimentally. Furthermore, future studies might address the relations of the variables in the present model with concepts of control, such as, self-efficacy (Bandura, 1986) and perceived behavioral control (Ajzen, 1988), and concepts of social influence, such as, subjective norm (Ajzen, 1988). The Self-evaluation Motivation Model, which is based on Bandura's (1986) Social Cognitive Theory, is one step in an attempt to unravel the low motivation to quit of many smokers and, especially, to provide a fruitful way to motivate them to change their lethal behavior. 


\section{Part II}

\section{The effectiveness of computer-tailored}

\section{interventions}




\title{
Chapter 5
}

\section{The development of computer-generated tailored interventions ${ }^{1}$}

\begin{abstract}
Tailoring information to individual characteristics of a person is a promising line of development of self-help interventions. This article presents a methodology for developing computer-generated tailored interventions. The first phase of the development concerns the formulation of intervention objectives on the basis of analyses of the cognitive determinants of behavior. The second phase concems the core of the development of a tailored intervention. For each objective, a socalled tailoring matrix is developed which specifies the individual characteristics to which the message will be adapted to. The tailoring matrices are the basis of the tailored messages and the tailoring questionnaire which will assess the individual characteristics. In the third phase, all the separately written messages must be integrated to one coherent intervention text and the lay-out is designed. This methodology may result in largely different tailored interventions depending on the theoretical framework used, the adaptation of the messages and the inclusion of feedback.
\end{abstract}

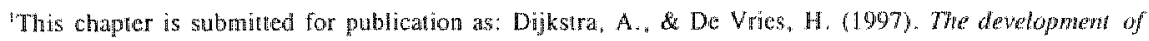

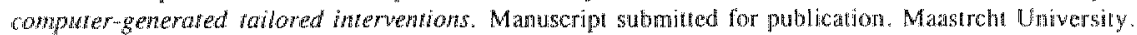




\section{Introduction}

The large scale application of health education materials has resulted in health education interventions which are directed at everyone but at no one in particular. One major drawback of these standardized interventions is that they contain information which in many cases is not applicable to an individual's psychological state or situation. Such a lack of relevance of intervention messages is considered to be detrimental to the effectiveness of an intervention.

Tailoring information to individual characteristics of a person is a promising line of development of self-help interventions, which brings back an essential part of the clinical approach: with the help of foreknowledge of a person or a group of persons, the content of tailored interventions is adapted to the relevant characteristics of an individual which are measured (De Vries, Willemsen, Brug, Dijkstra \& Berben, 1995; Strecher, Kreuter, Den Boer, Kobrin, Hospers \& Skinner, 1994; Velicer, Prochaska, Bellis, DiClemente, Rossi, Fava \& Steiger, 1993). Relevant characteristics can be regarded as those characteristics which influence the efficacy of the intervention. For example, one central and relevant characteristic which an intervention message can be adapted to is the motivational stage (Dijkstra, De Vries, Roijackers \& Van Breukelen, in press-d; Prochaska, DiClemente \& Norcross, 1992). Some interventions take into account only one relevant characteristic of a group of individuals, such as age (Rimer et al., 1994) or motivational stage (Pallonen et al. 1994), and sometimes the content of the intervention is adapted to several characteristics of an individual. The present article is concerned with this latter type of tailoring, in which, because of the complexity of the relations between several characteristics, the intervention messages are composed by a computerized tailoring system (De Vries et al., 1995; Strecher et al., 1994; Velicer et al., 1993).

Computerized tailored health interventions have been shown to lead to more behavioral changes compared to no information (Campbell, DeVellis, Strecher, Ammerman, DeVellis \& Sandler, 1994; Dijkstra et al., in press-d; Strecher et al., 1994) and compared to similar but standardized interventions (Brug, Glanz, Van Assema \& Kok, 1996; Brug, Steenhuis, Van Assema \& De Vries, 1996; Skinner, Strecher \& Hospers, 1994; Strecher et al., 1994).

In attempts to develop more effective tailored interventions, insight is needed into why tailored interventions work better than non-tailored interventions. Knowing the working mechanisms of tailored interventions would offer the opportunity of exploiting them further. Some suggestions are made about working mechanisms in the literature on tailoring. Tailored information might be more effective because it increases attentiveness to the message and it increases the relevance of the message (Kreuter \& Strecher, 1996; Skinner et al., 1994; Strecher et al., 1994). The finding that participants receiving a tailored message remembered it more often and stated to have read more of it (Campbell et al., 1994; Strecher et al., 1994), is congruent with this notion on working mechanisms.

Both the complexity of adapting information in an intervention to several 
characteristics of an individual and the need to develop effective lailored interventions based on theoretical and empirical insights, call for a lool in developing tailored interventions. The present article attempts to offer a stepwise structure in which a tailored intervention can be built. In the Preparation phase, the problem behavior and its determinants are studied and the intervention objectives are formulated. In the Tailloring phase, the decisions to adapt the information to individual characteristics are made and the adapted messages are written. Finally, in the Integration phase, care is taken that the composition of messages addressing different topics is a coherent and attractive whole.

\section{Phases in developing a tailored intervention}

In the case of individual health counseling, the counselor's expert view determines the response to the individual's psychological state or behavior. Similarly, tailored interventions will respond to relevant individual characteristics, on the basis of expert knowledge that is put into the tailoring system. The present outline of a tailoring methodology gives a global structure to transfer the expert knowledge into the computerized tailoring system (Figure 5.1). Although there is a functional order in the tasks to be accomplished, a realistic developmental process may take steps forwards and backwards. The application will be illustrated for the problem of smoking cessation. The present structure is the result of the development of several tailoring systems which have been tested in the last four years (Dijkstra, De Vries \& Roijackers, in press-a; Dijkstra, De Vries \& Roijackers, in press-b; Dijkstra et al., in press-d; Dijkstra, De Vries, Roijackers \& Van Breukelen, in press-e).

Although we intent to give a general framework in the development of a tailoring system, we do not presume that the following framework is universal, nor that it is the only possible framework. We present it as a possible methodology for developing computer-generated tailored interventions and aim to enhance the reader's insight in the potentials of this relatively new way of communicating health information. 


\section{Phase 1 PREPARATION}

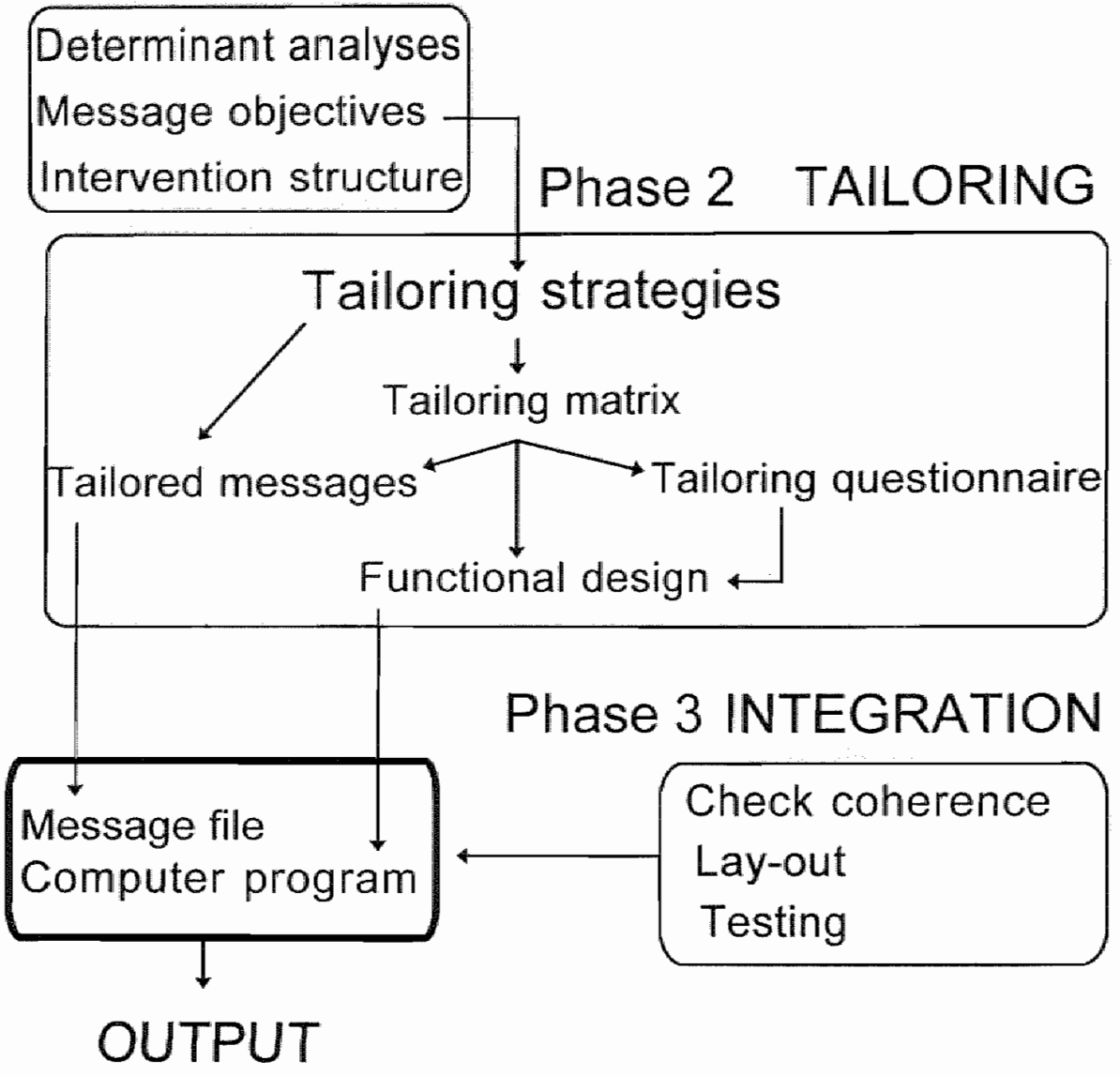

Figure 5.1 Three phases in the development of computer-tailored interventions. 


\section{Phase 1 Preparation}

This first phase is concerned with the question of what information or knowledge people should have in onder to become motivated and to be able to change their behavior. Therefore, the influence of psychosocial factors on smoking and smoking cessation should be studied. The psychosocial factors, or determinants, which are related to smoking and smoking cessation will, in turn, be translated into specific intervention objectives.

\section{Intervention objectives}

The first step in deciding what information people need to change their behavior is to assess the psychosocial determinants of the healthy behavior. These determinants can be translated in intervention objectives which can be formulated in terms of desired cognitive states or desired overt or covert behavior. For example, if the psychosocial determinant analysis reveals that the perceived long-term health consequences of quitting are predictive of abstinence, at global objective concerning these consequences could be: "The individual acknowledges that the personal risk of long-term consequences of smoking will decline in the case of quitting". If the psychosocial determinant analysis reveals that the perceived risk of lung cancer is related to quitting, an objective concerning this risk could be: "The individual acknowledges the increased risks of lung cancer due to smoking and the positive effects of quitting on this risk".

Thus, first, the psychosocial determinants of the healthy behavior have to be assessed. In principle this can be accomplished by combining two sources: 1) a theoretical model; 2) empirical data on the (un) healthy behavior. The following theoretical models are often used in health education and, thus, can serve as a frame of reference: the Health Belief Model (HBM; Rosenstock, 1974), the Theory of Planned Behavior (TPB; Ajzen, 1988), the Attitude-Social influenceEfficacy Model (ASE; De Vries, Dijkstra \& Kuhllman, 1988), ProtectionMotivation Theory (PMT; Maddux \& Rogers, 1983) Bandura's Social Cognitive Theory (SCT; 1986) and the Trans Theoretical Model (TTM; Prochaska et al., 1992).

All these models map psychosocial determinants. For example, when using SCT, three sorts of outcomes are defined: physical outcomes, social outcomes and self-evaluative outcomes (Bandura, 1997). Furthermore, SCT defines selfefficacy as a major determinant of behavior. Information in health education interventions is considered to influence behavior by changing the psychosocial determinants of behavior. On the basis of SCT, the intervention should include information on the three sorts of outcomes and information to influence selfefficacy. However, the health behavior models only offer an abstract and general description of the causes of behavior and behavior change. Therefore, empirical data are needed to operationalize the psychosocial determinants. For example, while SCT defines physical outcomes of smoking, empiricall data distinguish between short-term and long-term outcomes (Dijkstra et al., in press-a; Dijkstra, 
Roijackers \& De Vries, in press-c). Furthermore, while SCT defines self-efficacy expectations with regard to quitting, empirical data show that different clusters or domains of self-efficacy expectations in smoking cessation can be distinguished (Dijkstra et al., in press-a; in press-c; Velicer, DiClemente, Rossi \& Prochaska, 1990). While SCT defines social outcomes of quitting, empirical data show that the people closest to the individual are perceived to give the largest social rewards (Dijkstra, Bakker \& De Vries, 1997).

In sum, first, a theoretical model of the psychosocial determinants of smoking and smoking cessation is chosen. Second, the psychosocial determinant analysis is conducted and, third, the findings are translated into intervention objectives.

\section{The structure of the intervention}

To be able to develop a coherent tailored intervention, it helps to cluster related objectives. This facilitates a clear overview of the warious sections in a tailored intervention. This may result in a hierarchical structure consisting of chapter objectives, section objectives and message objectives. Table 5.1 shows an example of objectives at the level of sections. These section objectives define the desired states to be brought about by specific sections within the intervention. Depending on the findings of the psychosocial determinant analyses, more specific (lower level) message objectives might be formulated. For instance, the desired cognitive state with regard to the long-term health consequences of quitting may be translated into more specific message objectives about, for example, a desired cognitive state with regard to: 1) the risk of lung cancer; 2) the risk of CHD and; 3) the risk of COPD, due to smoking. In sum, an intervention should contain information to bring about the desired state described in the chapter objectives. Chapters will have to contain information to bring about the desired state described in the section objectives while sections will have to contain information to bring about the desired state described in the (lowest level) message objectives.

Besides structuring the intervention on the basis of related clusters of objectives, some additional considerations are important for ordering the content of the intervention. First, we can formulate intervention objectives which define the desired cognitive state smokers need to attain to understand the information offered in the tailored intervention. For example, the intervention should explain the goal and the rationale of the information which is offered. Second, considerations with regard to the sequence of arguments and information (e.g. prinacy and recency effects) and the different sorts of information may partly determine the structure. 
Chapters and sections

1. Mtroduming

-Goal of the intervention

- Pationale of the contert

\section{Regison for smoking}

- Relatation

\section{- Soctalizing}

2. Reason for quitumg;

mong-term healh consequences

Whort-term health consequences

- Social consequences

- Sellievaluative consequences

1. Coping with the lurge to smoke

Coping with negative emotions

Coping with social pressure

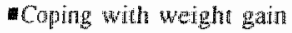

Copinge will redapse

-Coping with AVE

Concluding saction

- Goal of the interwention
Section objectives

The indingual:

linerprets the contert information in the contex of the gual of the intervention.

Knows why the spectic sorts of intornation are needed to reach the gonl of the interwention.

Knows ihat smokers are psychologically dependent on smoking and reognzes over and cover stgris of psychological dependence. Knows that physical dependence leads to whindrawal symptoms in the case of quitring. Knows that the presumed relaxing qualities of smoking are due to the relief from withdrawal symptoms.

Knows that smoking cigarenes is not a sochal lubrican, but rather hint smokers light a cigarette when they are socializing. Knows that the expectations that smothing will lead to more satisfactory socializing causes craving in social sinuaions.

Knows that the personal risk of long-term health consequences of smoking will decline in the case of quitting.

Knows the short-term health conseguences of snoking, is able to adewuately atribute his or her physical symptoms to smoking and knows the shorim then health benefits of quitting.

Krows that smoking can be inconvinient for people in the social anwromment, bad for their health and that snoking behavior models smoking for youngsiers. Knows the social benetirs of quitting.

Knows that smoking is discrepant with persona shandards of goals. Recognizes foctings of negative selfevaluaton and knows the self fevaluative rewards of quitring.

Knows that cigarettes do not contain a substance that reduces negative emothons. Is able to anticipate negative emotions and to use allemative ways to cope with a depressed mood, anger and feelings of stress.

Is able to anticipate social risk situations and to cope with thent. Is able to "say no" when a cigarette is offered, is able to join non-smokers on a party, is albe to make a social commitment to guat, elt.

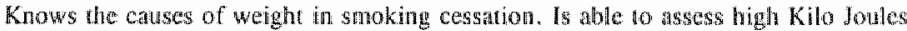
products in this or her diet. Is able to buy low Kilo Joules produch and is able to exersise on regular basis

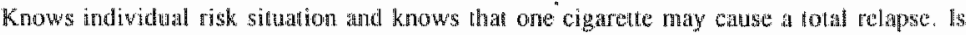
able to anicipate risk situations. lis able to recognize fectings of guill after a lapse. Is albe to leam from a lapse and not give up.

Interptets the content infortation in the contex of the goal of the indurvention. 


\section{Phase 2 Tailoring}

Phase 2 concerns the core of the development of a tailored intervention. In this phase decisions are made to what extent the computer-tailored intervention will be tailorcd. That is, to what extent people with different characteristics need different messages in order to bring about the desired state defined by the message objective. The adaptation of messages for people with different characteristics is meant to enhance the effectiveness of the information and is based on different tailoring strategies. Tailoring strategies are ways of tailoring messages.

One tool in this phase is the tailoring matrix which needs to be developed for each message (or lowest level) objective. The tailoring matrix depicts the characteristics which the content of the message is adapted to. The tailoring matrix is based on the inclusion of tailoring strategies.

This phase results in three products which are all directly related to the tailoring matrix. First, this phase results in the tailoring questionnaire which assesses the individual characteristics which are taken into account in the tailored intervention. Second, this phase results in the decision rules (functional design) which are included in the computer program. Third, this phase results in the message file from which the computer program composes the tailored intervention.

\section{Tailoring strategies}

At this point, decisions have to be made to what extent the message should be adapted to individuals with specific characteristics. The message objective formulates the desired cognitive or behavioral state of the individual. The question which arises is: "Do we have to adapt the content or framing of the message to people with certain characteristics, in order to bring about the desired state?". The decisions to adapt messages to people with certain characteristics are based, ideally, on additional research and in-depth analyses of the (psychosociai) determinants of behavior and behavior change. The adaptations of messages is thought to be partly responsible for the surplus value of the tailored intervention compared to a non-tailored intervention. In other words, adaptations of messages are the strategies which are used to increase the effectiveness of the information offered. Table 5.2 and 5.3 show clusters of tailoring strategies: adaptation of the message (Table 5.2) and feedback (Table 5.3). 


\begin{tabular}{|c|c|c|}
\hline Strategy & Effect & Example \\
\hline $\begin{array}{l}\text { 1. Wess redundant } \\
\text { information }\end{array}$ & Entanced anterntion & 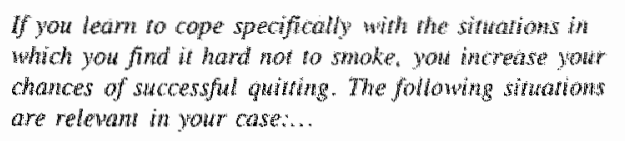 \\
\hline $\begin{array}{l}\text { 2. More relevant } \\
\text { information }\end{array}$ & Enhanced attention & 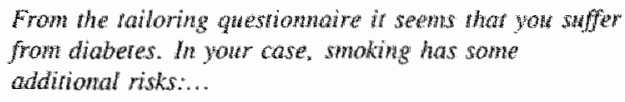 \\
\hline 3. More information & More persuasive argiments. & $\begin{array}{l}\text { Because less redundant information is offered, there is } \\
\text { more room for other (and more relevan) information }\end{array}$ \\
\hline $\begin{array}{l}\text { 4. Avoiding } \\
\text { defensiveness }\end{array}$ & Enhanced attention & 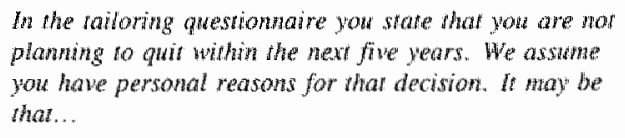 \\
\hline $\begin{array}{l}\text { 5. Personal goal- } \\
\text { seting }\end{array}$ & Enhlanced self-regulation & 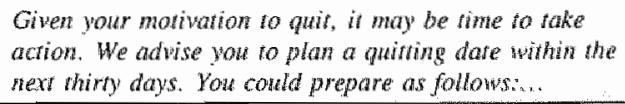 \\
\hline
\end{tabular}

Adaptation of the message. These five tailoring strategies are used in interaction with the development of the tailoring matrix and, hence, in interaction with decisions to include certain characteristics in the tailoring matrix. First, a message could be adapted to the foreknowledge of an individual on a certain topic. In this way, the adapted message contains less redundant information. This is thought to enhance the attention to the information offered. Second, a message might contain information that is only relevant for specific individuals. For example, information on the cumulative risk of smoking and diabetes is only relevant for diabetic smokers. Third, because the inclusion of information in messages in this way is more efficient, the message can contain more extensive information. Fourth, a message might be adapted to, for example, the individual's low motivation to change. It is expected that in this way defensiveness, and the resulting counter argumentation, could be avoided. Fifth, the message might be adapted to the phase of the behavior change process. For example, such a message can offer an individually matched behavior goal.

However, we could include these five tailoring strategies to adapt the content and framing of a message without the individual knowing that the message is specifically targeted at him or her. Inclusion of personal feedback may make a person aware of the fact that the intervention is personalized. 


\begin{tabular}{|c|c|c|}
\hline Siralcgy & Erfect & Example \\
\hline $\begin{array}{l}\text { 1. Ferdback on } \\
\text { cognilivebetsavioral } \\
\text { sitates }\end{array}$ & Enhanderat self-nonitoring & $\begin{array}{l}\text { In the toitoring questionnaire you say: "I am wor } \\
\text { ashamed thay I thave" to smoke"... }\end{array}$ \\
\hline $\begin{array}{l}\text { 2. Normanive } \\
\text { redback }\end{array}$ & Eriflanced sociol comparison & $\begin{array}{l}\text { Compared to orier smolkers who are motivated to quit, } \\
\text { yotir confidence in your ability to quit is faworable.. }\end{array}$ \\
\hline 3. Posithe feedback & $\begin{array}{l}\text { Retinforcement of desired } \\
\text { states }\end{array}$ & $\begin{array}{l}\text { In the lailoring questionnaire you report that you have } \\
\text { refrained from smoking for seven days. This is fantastit, } \\
\text { keep up the good work! }\end{array}$ \\
\hline $\begin{array}{l}\text { 4. Fed back on } \\
\text { personal characteristics }\end{array}$ & $\begin{array}{l}\text { Enhanced curiosily and } \\
\text { involvemena }\end{array}$ & $\begin{array}{l}\text { Mrs. Taylor, you srate that you are nor planning to gais } \\
\text { within the nex five years. If you ketg on smoking } 17 \\
\text { cigarettes a doy, you wrill smoke anlother } 31.025 \\
\text { cigarentes. }\end{array}$ \\
\hline $\begin{array}{l}\text { 5. Personal tone and } \\
\text { empathy }\end{array}$ & Enhanced commiment & $\begin{array}{l}\text { Dear Mr. Taylor, we understand your concerns about } \\
\text { quituing and we understand that you are worried abour } \\
\text { the health problems you have. You must feel trapped. }\end{array}$ \\
\hline 6. Apsative feedback & Enthanced self-monitoring & $\begin{array}{l}\text { Compared to our last contact swith you, you anticipare } \\
\text { more pros of quatring... }\end{array}$ \\
\hline $\begin{array}{l}\text { 7. More than one } \\
\text { a 'cue to action"* }\end{array}$ & $\begin{array}{l}\text { Stimulating attentional } \\
\text { change processes }\end{array}$ & $\begin{array}{l}\text { Sutsequent and related tailored interventions may be } \\
\text { offered wice or more often to an individual. }\end{array}$ \\
\hline
\end{tabular}

* Only applicable in multiple sailoring

Feedback. Feedback constitutes the second cluster of tailoring strategies that may be included in tailored interventions. The five sorts of feedback have in common that a characteristic state of an individual or a response to such a state is fed back. The first type of feedback concerns information on scores on cognitive variables, such as, expected outcomes and self-efficacy expectations, and behavioral accomplishments. For example: "In the questionnaire you state that....", or "Since the last measurement, you have been cutting down on cigarettes". The second type of feedback is normative feedback. The tailoring system may compare individual states with those of comparable others. In this kind of feedback, it is especially important to assure that the feedback is not detrimental to the process of behavior change. For example, if an individual has very low confidence in the ability to change behavior compared to others, feeding back this information might further diminish the confidence. The third type of leedback concerns a reinforcing reaction to the individual's state. A state which is considered to be beneficial to behavior change may be reinforced, whereas if a state is detrimental to behavior change it might be ignored. For example, if a person is preparing a quit attempt, this plan may be reinforced in the message. The fourth type of feedback concerns information on personal characteristics, such as, the quit history, a person's name or the average number of cigarettes a day. In principle, all kinds of relevant or non-relevant characteristics might be fed back, as long as they serve the function of personalizing the message and as long as they do not interfere with the behavior changing power of the message. The fifth type of feedback concerns the 
application of a personal tone and an empathic reaction to the individual's state. For example, before offering restructuring information, understanding could be shown if an individual states: "I will not be a better example for the people around me if I quit smoking". Furthermore, an empathic reaction could be given when an individual states: "The thought of never smoking again scares me". It is of central importance to relate to the subject's experience (Miller \& Rollnick, 1991).

An additional type of feedback is ipsative feedback which can only be offered in multiple tailored interventions. This is feedback on the extent and direction of changes in relevant characteristics. For example, if an individual's confidence increases between two assessments, this is fed back. As in nomative feedback, care has to be taken that the feedback is constructive.

\section{Tailoring matrices}

A tailoring matrix is based on decisions to include certain tailoring strategies. It depicts the characteristics which the content of the message is adapted to. The tailoring matrix summarizes the characteristics that have to be assessed with the tailoring questionnaire. It also describes the specific configurations of characteristics that lead to a certain message. As such, it depicts the decision rules which are the basis of the tailoring computer program. For example, Table 5.4 shows the tailoring matrix related to the message objective about the desired cognitive state with regard to the risks of CHD due to smoking. The following considerations were used. First, sex was regarded as a relevant characteristic because information on the additional risk of CHD through the combination of smoking and the use of oral contraceptives was thought to be only relevant for females. Second, age was regarded as a relevant characteristic because mainly younger women use oral contraceptives and, in general, youngsters may need a different framing of information on the risks of CHD. Third, foreknowledge of CHD and the extra risks of using oral contraceptives was regarded to be a relevant characteristic because extended foreknowledge could make the message redundant. Fourth, the intention to quit was regarded to be a relevant characteristic because the framing of the message for smokers with low motivation must be designed to specifically avoid resistance in the reader. Thus, the tailoring matrix in Table 5.4 distinguishes between 24 messages (i.e. 2 (sex) X 3 (age levells) X 2 (intention levels) X 2 (high/low foreknowledge)). Not all 24 messages have to be inherently different. It may be that the message for males older than 60 with high intention and high score on foreknowledge does not differ from the message for comparable females. Furthermore, information on the additional risks of using oral contraceptives is addressed in the messages aimed at women in the two younger age groups (messages $4,5,10,11,16,17,22,23)$, but not in the message for women in the older age group (messages 6,12,18,24). All the tailoring matrices together describe the extent to which the intervention is tailored. 
Talong matrix of the message objecrive with regand to the rists of CHD with sex, age. Hatemion, for elwowledge as relevan characieristics.

\begin{tabular}{|c|c|c|c|c|c|}
\hline & \multirow[b]{2}{*}{ A } & \multicolumn{2}{|c|}{ - High intention to quit } & \multicolumn{2}{|c|}{ - Low intention to quit } \\
\hline & & $\begin{array}{l}\text { High score on } \\
\text { foreknowledge } \\
\text { with regard to } \\
\text { whe risk of CHD }\end{array}$ & $\begin{array}{l}\text { Low score on } \\
\text { foreknowledge } \\
\text { with regard to } \\
\text { the risk of } \mathrm{CHD}\end{array}$ & $\begin{array}{l}\text { High score on } \\
\text { foreknowledge } \\
\text { with regard to the } \\
\text { risk of } \mathrm{CHD}\end{array}$ & $\begin{array}{l}\text { Low score } \\
\text { foreknowledg } \\
\text { with regard to } \\
\text { risk of } \mathrm{CHD}\end{array}$ \\
\hline \multirow[t]{3}{*}{ Mate } & $<25$ & message i & message 7 & message 13 & message 19 \\
\hline & 251055 & messige 2 & message 8 & messiage 14 & message 20 \\
\hline & $>55$ & message 3 & message 9 & message 15 & message 21 \\
\hline \multirow[t]{3}{*}{ - Female } & $<25$ & nessage 4 & message 10 & message 16 & message 22 \\
\hline & 251055 & message 5 & message 11 & message 17 & nessage 23 \\
\hline & $>55$ & message 6 & message 12 & messinge 18 & message 24 \\
\hline
\end{tabular}

\section{Tailoring questionnaire}

To be able to tailor the intervention to individual characteristics, these characteristics need to be assessed. Therefore, a special tailoring questionnaire is necessary. Some specific considerations are taken into account in the development of this questionnaire.

First, the questions have to assess the characteristics in a valid way. That is, it must be clear what score on a certain item refers to a certain state with regard to the characteristic to which the message is adapted to. For example, it may be difficult to decide what information an individual should be offered if he or she answers: "I agree somewhat". In contrast, an answer such as: "Smoking cessation only decreases the risk of lung cancer slightly", might be more easily interpreted.

Second, the formulations of the questions in the tailoring questionnaire might take into account whether the characteristic is fed back to the individual. That is, individuals will have to accept the information as being valid and referring to their personal state or situation. For example, with regard to the assessment of the anticipation of long-term physical consequences of quitting (foreknowledge) with a 7-point scale from "not at all" to "very much", an individual score of 6 may be hard to feed back usefully. That is, feeding back "you scored 6 on that scale" is thought to express little. In contrast, feeding back an answer such as "smoking cessation decreases the risk of lung cancer very much", is thought to be more expressive and to be more easily recognized as an individual's statement. 
The combination of the tailoring matrices and the tailoring questionnaire is translated into the functional design which is then translated in a computer program by a professional programmer. The functional design contains the following information: 1) "if...then.." statements which relate certain configurations of characteristics to the appropriate tailored messages; 2) computations to construct composite measures; 3) algorithms (including cut-off points) to produce normative feedback; 4) handling of missing data; 5) data entry rules relating data and variables.

\section{Phase 3 Integration}

Coherence. In this phase, all the separately written messages must be integrated to one coherent intervention text. For example, in principle all messages on the short-term health consequences must fit after the message on the long-term consequences of smoking. Statements, such as, "you have to decide for yourself", and feedback, such as "you are on the right track", should be given in limited amounts. Thus, in writing one message, the content and framing of all other possible messages must be taken into account.

Lay-out and output. In this final phase the lay-out is developed. General principles of attractiveness and readability are applied. For example, important or recognizablle personal statements can be fed back and put in magnified characters. The lay-out partly depends on how the tailored information is offered to individuals. That is, tailored information may be communicated in many ways. For example, in a booklet format, in a letter format or using interactive video.

Computer program. The functional design is translated into the computer program which combines the input from the tailoring questionnaire with messages stored in the message file. Furthermore, the computer program could include rules to choose shorter messages if the whole intervention exceeds a certain number of pages and to assure that on every page there is one cartoon despite the length of the messages.

Testing. Finally, the output has to be compared with the data which are put in. Furthermore, fake-records (data from non-existing individuals) with extreme or unexpected scores might be used to test the output of the system. Although composed from different messages, the eventual tailored intervention needs to be one coherent, attractive and, above all, consistent tailored intervention.

\section{Conclusion and discussion}

The development of a tailoring system requires extensive knowledge of principles of communication and behavior change. The present outline of a tailoring methodology is meant to serve as a global structure for putting expert knowledge on principles of communication and behavior change in a tailoring 
system.

Several decisions are made while developing the system which influence the eventual type of tailored intervention. First, the theoretical model and the related structure determine the intervention. Different theoretical models may map different psychological constructs and, hence, the intervention may target different psychosocial determinants. For example, in the Health Belief Model (HBM; Rosenstock, 1974), risk perception is one of the determinants of health behavior. A tailored smoking cessation intervention based on HBM would explicitly target smokers' risk perception. In the Attitude-Social influenceEfficacy Model (ASE; De Vries et al., 1988) social influence is conceptualized with three constructs: social norm, modeling and social pressure (De Vries, Backbier, Kok \& Dijkstra, 1995). A tailored intervention based on the ASEmodel would explicitly aim at changing the individual's social norm, the modeling influences and the perception and coping strategies with regard to social pressure.

A second factor that influences the eventual type of tailored intervention is the adaptation of the message and the inclusion of feedback. The adaptation of the messages is based on the sort and the number of characteristics to which it is adapted. These decisions determine the extent to which the tailored intervention is tailored. A tailored intervention may take into account only one characteristic, for example, stage of change or foreknowledge, or it may take into account many characteristics. Furthermore, the desired cognitive or behavioral state described in the message objectives may be instilled or reinforced in different ways. For example, a message objective might be, "The individual has high self-efficacy to cope with emotional situations". This objective might be addressed by offering coping skills in the message but also by offering normative feedback which could be offered in different format, such as, a graph. With regard to offering feedback, interventions may also greatly differ. All kinds of feedback may be included or, at the other extreme, no feedback could be given. The feedback may be on the item level (e.g. "In the questionnaire you said ....") or the construct level ("with regard to the advantages of quitting you score...."). Furthermore, the intervention might be personalized to a certain degree by leeding back recognizable individual characteristics, such as, the name and the number of cigarettes a day.

Thus, greatly different tailored interventions can be developed using the present methodology. Future research will have to determine which decisions lead to the most effective tailored intervention. At present, the understanding of the sometimes superior effectiveness of tailored interventions is still in its infancy. First of all, we do not know why tailoring sometimes works better than non-tailoring. Hence, research needs to address the working mechanisms of tailoring in dismantling studies. Knowing why tailoring works may lead to further exploitation and development of working mechanisms in tailoring strategies. In the present article we distinguish between two sorts of working mechanisms.

The first cluster of working mechanisms is related to the adaptation of the content of the messages to individual characteristics. This form of tailoring can 
be applied without the individual consciously knowing that the message is tailored. Skinner et al. (1994) report that a tailored intervention which was tailored in content but written as if it were a general intervention, was indeed more effective than a similar looking but standardized intervention. In other words, the adaptation of the content of messages seemed to have a behavior changing power of its own.

The second cluster of working mechanisms consists of different forms of feedback. Feedback is a basic construct in cybernetics and serves an essential function in psychological self-regulation models (Bandura, 1986; Bertalanfy, 1968; Powers, 1973). The extent to which both sorts of working mechanisms are responsible for behavior change could be tested in a dismantling or constructive treatment strategy (Kazdin, 1986). In these treatment evaluation strategies, essential ingredients are withdrawn or added, respectively, over different experimental conditions.

Besides these fundamental questions, future research could address the application of taillored interventions in the health care system. The first issue in the application of tailored interventions concerns how to reach people who engage in the target behavior. In principle, two recruiting strategies seem important, reactive and pro-active recruitment. In reactive recruitment, the possibility of receiving tailored self-help materials can be disseminated via the media. This might be accomplished by posters in health care institutions, such as hospitals, a GP's office or pharmacies. Furthermore, the availability of tailored self-help materials could be published in magazines and newspapers or broadcast on television.

In pro-active recruitment, tailored self-help materials might be offered to all people who are identified to engage in the target behavior. In principle, all health care professionals who meet people from the target population might be active in distributing the materials. For example, GPS, medical specialists, nurses or pharmacists might be in a position to identify the members of the target population. Pro-active recruitment leads to broad dissemination of self-help interventions. Another benefit is that the health advice of health care professionals can be backed-up by a printed expert view and advice on behavior change.

A second issue in the application of tailored interventions is the individual assessment of the personal characteristics. Their are several ways to gather the individual data. The questionnaire might be printed and șent by mail to the organization which processes the data and produces the tailored intervention. The questionnaire might be presented on a computer screen and directly fed into the tailoring system. For example, the distribution of tailoring questionnaires and tailored self-help materials through the World Wide Web might have future potential.

A third issue in the application of tailored interventions is the medium which is used to communicate the tailored information. In principle, tailored self-help materials might be offered on a television/computer screen or printed. It seems of importance to recognize the typical communicative characteristics of the 
different media. For instance, information offered on a television screen may be more volatile than information printed on a page. Once the television is turned off, the information, and maybe the behavior changing appeal, vanishes. Furthermore, the interpretation of the information offered through one medium may be distorted by the normal function of that medium. For example, the information given on a television screen may be more associated with entertainment than with a reliable source of information.

In terms of cost-effectiveness, future studies will have to show which recruitment strategies, which ways to distribute the tailoring questionnaires and which ways to offer the tailored information are the best.

In conclusion, the present methodology may lead to greatly different tailored interventions, although the issues on the effectiveness and the applications of the interventions are relevant to them all.

Finally, although the development of a tailoring system may be time consuming, once the system is operational, it can be used for many years. Whereas standardized self-help materials are printed in large numbers and continue to be distributed even when new facts about the target behavior are discovered, interventions produced by a computerized tailoring system can be easily updated. The messages in the message file can be retyped using a word processor and new insights into relevant individual characteristics can be included in the system by adding decision rules to the functional design and incorporating these rules into the computer program. In other words, once a tailoring system is built on a solid theoretical foundation, it can be used and updated for years. 


\title{
Chapter 6
}

\section{Tailored interventions to communicate stage-matched infor- mation to smokers in different motivational stages'.}

\begin{abstract}
Smokers in stages of low readiness to quit (immotives and precontemplators) and smokers in stages of high readiness to quit (contemplators and preparers) were randomly allocated to one of four tailored intervention conditions offering: 1) outcome information, 2) self-efficacy enhancing information, 3) both sorts of information or 4) no information. The data on 1540 smokers were analyzed, stratified by stage. The primary outcome measure was stage transition.

In immotives, both conditions containing outcome information led to more transitions than the control condition, whereas in precontemplators, both conditions containing self-efficacy enhancing information led to more transitions than the control condition. A more stringent match-mismatch test (i.e. the comparison between immotives and precontemplators who were assigned to the matched treatment with immotives and precontemplators who were assigned to the mismatched treatment) did not verify the hypotheses with regard to stage-matched information. With regard to contemplators and preparers, the following was found: compared to the control group, contemplators benefitted the most from the condition containing both sorts of information whereas preparers benefitted the most from the condition containing self-efficacy enhancing information only. The match-mismatch test supported these findings in contemplators and preparers.
\end{abstract}

"This chapter will be published as: Dijkstra, A. De Vuies, H, R Roijackers, D., Van Breukelen, G. (in press). Tailored interventions io communicate stagematched information to smakers in different motivational stages. Foumal of Constuling and Clinicat sychology. 


\section{Introduction}

Self-help interventions in smoking cessation may be useful in lowering the percentage of smokers in the population (Lichtenstein \& Glasgow, 1992). However many smokers are not interested in such interventions which are mostly directed at people who are intending to quit smoking. In the Netherlands $70 \%$ of smokers are not planning to quit within the next six months (Mudde, Dolders \& De Vries, 1994). It is conceivable that the impact of an intervention on the smoking rate in the population may be enhanced by also targeting this large group of smokers.

The stages of change model (Prochaska, DiClemente \& Norcross, 1992) explicitly recognizes this group of smokers with low readiness to change. The model views behavior change as a process from no readiness to change the behavior to internalization of the new behavior and it distinguishes between several discrete stages of behavior change. With regard to interventions, first, a stage-matching principle can be derived from the stages of change model: different interventions have to be directed at each stage. Second, the aim of an intervention should be stage transition.

From a theoretical point of view, stage-matched interventions will have to be directed at changing the cognitive determinants which are responsible for the transition from one stage to another. On the basis of earlier (crosssectional) studies, it was suggested that smokers with low readiness to quit especially need information on outcomes to progress to the next stage, whereas smokers with bigh readiness to quit especially need self-efficacy enhancing information (De Vries \& Backbier, 1994; Dijkstra, De Vries \& Bakker, 1996). Similarly, according to Janis and Mann (1977), in a sound decision making process, people first process information on the consequences of changing or not changing the behavior and after that they process information on how to change the behavior (Also see Miller \& Rollnick, 1991).

A literature search revealed no study on the differential impacts of outcome information and selfefficacy enhancing information on behavior change. Especially in the development of stage-matched interventions, there is a need for data on the impact of both sorts of infornation in interaction with stage of change.

In the present study, an extended version of the original stages of change model was used (Dijkstra, Bakker \& De Vries, 1997). Within the group of smokers who are not planning to quit within the next six months, smokers in two stages were recognized: immotives, who are not planning to quil within the following five years (or planning to never quit), and precontemplators, who are planning to quit within the following five years (but not within the following six months). Whereas precontemplators are considered to be "on their way" to the next stage, immotives are considered to be "stuck" in low readiness. Within the group of smokers who are planning to quit within the next six months, also, smokers in two stages were recognized: contemplators, 
who are planning to quit within the next six months but not yet within the next month, and preparers, who are planning to quit within the next month (DiClemente et al., 1991). Whereas contemplators are considered to only think about quitting, preparers have decided to quit on the short term. Hence, four groups of smokers with increasing readiness to quit were distinguished: immotives, precontemplators, contemplators and preparers.

With regard to stage-matched interventions, the findings from De Vries and Backbier (1994) and Dijkstra et al. (1996) were extrapolated to the stage model used here. With regard to both extreme groups, immotives and preparers, the expectations were as follows: immotives would benelit most from outcome information only (why quit), whereas self-efficacy enhancing information (how to quit) would even work detrimental by triggering defensive information processing. That is, the strong behavior changing appeal of the latter sort of information is largely mismatched with their low readiness to quit. Preparers would benefit most from self-efficacy enhancing information, whereas outcome information would be highly redundant and therefore inhibit information processing. It was expected that smokers in the intermediate groups, precontemplators and contemplators, would benefit most from the combination of both sorts of information, whereas one of both sorts of information would not be enough to stimulate stage-transition.

The first goal of this study, which was of mainly practical value, was to assess what sort of information smokers in each stage have to be offered in smoking cessation interventions. Therefore, first, the effectiveness of three experimental conditions were compared, for each stage, to that of a noinformation control condition. The conditions offered: 1) outcome information only or; 2) self-efficacy enhancing information only or; 3) both sorts of information. Second, the experimental conditions were compared to each other.

The second goall of this study was to assess whether the principle of stagematched information - smokers in different stages need different sorts of information - could be demonstrated. Therefore, smokers who were assigned to a stage-matched condition were compared to smokers who were assigned to a stage-mismatched condition.

The primary outcome measure was stage transition, because this measure is by definition an appropriate outcome measure for smokers in all stages (Velicer, Rossi, Prochaska \& DiClemente, 1996). In order to validate the results obtained with this measure, more conventional measures were additionally used: intention to quit and a point prevalence abstinence measure.

\section{Method}

\section{Participant recruitment}

Smokers were recruited by advertisements in local newspapers throughout the Netherlands. Two groups of smokers were explicitly invited to participate 
in this study: smokers who were planning to quit within the following six months (high readiness to change) and smokers who were not planning to quit within the following six months (low readiness to change). They were asked to volunteer for a research project on minimal interventions for smoking cessation including the possibility of being a control group member. Participants completing all three questionnaires were offered the opporturity of 10 bonus prizes amounting to $\$ 100$. After participants had phoned the university to register $(n=1733)$, they were sent the pre-test questionnaire which could be returned in a pre-paid envelope. After two weeks 1540 , pretest questionnaires $(88.9 \%)$ had been retumed (excluding sixty questionnaires from pipe or cigar smokers and questionnaires filled out incompletely).

\section{Design}

Smokers were randomly assigned to one of four conditions: Only information on outcomes of quitting ( $O C$ condition, $n=384)$; only self-efficacy enhancing information ( $\mathrm{SE}$ condition, $\mathrm{n}=385$ ); both sorts of information ( $\mathrm{BO}$ condition, $n=386$ ) or no information at all ( $\mathrm{CO}$ condition, $\mathrm{n}=385$ ). Participants in the experimental conditions were sent the intervention material by mail, while participants in the control condition received a letter in which they were told they would get no information. Two weeks (T2) and ten weeks (T3) after the intervention, participants were sent the post-test questionnaires. In this article, data from $\mathrm{Tl}$ and $\mathrm{T} 3$ will be presented. In addition, the data on the evaluation of the intervention from $\mathrm{T} 2$ will be presented.

\section{Tailored feedback}

The interventions consisted of computer generated individually-tailored intervention letters containing the condition-specific sort(s) of information. The computerized system to generate the tailored letters was adapted from a previous evaluation of a minimal intervention in smokers who were planning to quit (Dijkstra, De Vries \& Roijackers, in press-a). The present intervention consisted of a five- to seven-page computer-generated individualized report based on the pre-test questionnaire scores. Decision rules, developed in relation to the question: "What answer in the questionnaire leads to what information in the intervention?", combined several potential parts of the message to one coherent message. The reports in the three experimental conditions contained the specific sorts of information, as reported in the design section of this article, but were tailored to the individual's stage of change, perceived outcomes, situational self-efficacy levels and smoking behavior. In order to personalize the letters, the name of the individual was mentioned three times in each letter. Two specific measures were taken to guarantee standardization. First, to prevent the number of pages from exceeding seven, the expert system was programmed to use shorter messages if necessary. Second, although within one condition the contents of message 
parts varied depending on the individual scores, the sume topics were addressed in each letters.

$O C$ condition. In the introduction of these tailored letters it was stated that readiness is crucial in smoking cessation and that the letter was meant to enhance readiness by stressing the pros of quitting. The letters in this condition contained information on possible outcomes of smoking and quitting, such as personal health consequences, social consequences, such as appreciation of quitting by a nonsmoking partrer and the consequences of smoking for people in the environment. The amount of information depended on the individual scores on the items assessing the expected outcomes. Regarding monetary consequences, the amount of money saved after one month and one year of quitting was computed for each respondent. Restructuring information was offered on the negative outcomes of quitting, such as weight gain and loss of functions of smoking, such as relaxation and expected withdrawal symptoms. For example, if a smoker anticipated strong withdrawal symptoms, the temporal character of these symptoms was explained.

$S E$ condition. In the introduction of these tailored letters it was stated that the active use of skills to quit is crucial in smoking cessation and that the letter was meant to enhance confidence in quitting by offering skills which had proved helptiul to ex-smokers. The letters in this condition contained information on skills to cope with social, emotional and/or addictive situations, depending on the individual confidence scores. For example, skills, such as coping with cravings, stress, anger and a depressed mood, with withdrawal symptoms and social pressure were addressed. When a smoker reported a lot of confidence in being able to refrain from smoking in a specific situation, the smoker was told this increased his or her chances on success in quitting. When a smoker had moderate or little confidence in being able to refraining from smoking in a specific situation, cognitive and behavioral skills were offered.

BO condition. In the introduction of these tailored letters a rationale, similar to that in the other conditions, was offered stressing the importance of both sorts of information. The letters in this condition contained both sorts of information. All topics addressed in the OC- and SE condition, were also addressed in this condition.

\section{Measures}

Stages of change and stage transition. Stages of change were assessed by confronting smokers with different plans with regard to smoking cessation. Of seven quitting plans, they were asked to indicate "the one quitting plan which best fitted their own intention to quit". Smokers who indicated planning to quit within the following month were considered to be preparers. Smokers who indicated planning to quit within the following six months were considered to be contemplators. Smokers who indicated: 1) planning to quit within the following year or; 2 ) in the following five years, were considered to be 
precontemplators. Smokers who indicated: 1) planning to quit somewhere in the future but not within the following five years or; 2) planning to stay smoking indefinitely but planning to cut down or; 3) planning to stay smoking indefinitely and not planning to cut down, were considered to be immotives. The primary outcome measure was stage transition. Stage transition was assessed by dichotomizing changes in stage: forward transition was scored as 1 , versus no transition or backward transition which was scored as 0 . Participants were considered to be in action when they stated they were engaged in a quit attempt at the time of the measurement.

Intention. To verify the results obtained with the stage transition measure, two more commonly used outcome measures were used. First, intention to quit was measured with a composite of two 10-point scales: "Do you intend to quit smoking?" ("not at all" (1) to "definitely" (10)), and "How likely are you to quit smoking?" ("not likely at all" (1) to "very likelly" (10)). The intention score was the average of both item scores.

Point prevalence abstinence. Second, a more conservative criterion was used to assess smoking cessation: "Have you smoked in the last seven days? (even one puff)" (yes/no). No biochemical verification of the self-reported quitting behavior was conducted, because it was expected that the announcement of a biochemical verification would increase nonresponse and drop-out, especially in smokers who were not planning to quit within the following six months (see Velicer, Prochaska, Rossi \& Snow, 1992). This would lead to a highly selective group of immotives and precontemplators. Moreover, because half of the sample consisted of these smokers and only a three-month followup was planned, quitting smoking was not considered to be the most important outcome measure (see Velicer et al., 1992).

Pros and cons of quitting. Twenty-one items assessed the anticipated physical, social, self-evaluative and monetary outcomes of quitting. The items could be scored from "not sure" or "not expecting a certain outcome" $(0)$ to a "strong expectation of the outcome" (3). The items were derived from an earlier questionnaire, assessing the pros and cons of quitting, which was used in a study on the stages of change (Dijkstra et al.., 1996). In that study, the pattern of the pros and cons of quitting through the stages was almost identical to the pattem found with the Decisional Balance measures (Velicer, DiClemente, Prochaska \& Brandenburg, 1985). The present scale assessing pros of quiting was composed of 15 items referring to the positive outcomes of behavior change. Cronbach's a was .87. The scale assessing the cons of quiting was composed of six items referring to the negative outcomes of behavior change $(\alpha=57)$. The average item scores were used as the scale scores. Correlation between the scales was significant but low, $r=24$.

Self-efficacy expectations. Seven items assessed the confidence to refrain from smoking in various situations. The items were derived from a questionnaire used in a study on the predictive power of self-efficacy in smoking cessation (Mudde, Kok \& Strecher, 1995). Factor analyses revealed two factors: selfefficacy in coping with social situations ( 4 items, $\alpha=81$ ) and 
self-effcacy in coping with negative emotions (3 ittems, $\alpha=86$ ). All selfefficacy expectations were measured on a 7 -point scale and could be scored from "not sure at all that I am able to refrain from smoking" $(-3)$ to "very sure I am able to refrain from smoking" $(+3)$. The average item scores were used as the scale scores.

Smoking behovior and demographics. Smoking behavior was measured by asking smokers how many years they had been smoking, how many cigarettes they smoked on average and whether they had engaged in a 24 -hour quit attempt in the preceding 12 months. Heaviness of smoking was measured by the Fagerström Test for Nicotine Dependence (Heatherton, Kozlowski, Frecker \& Fagerström, 1991) which assesses smoking habits with six items. The item scores were added and the minimum possible score was 0 , the maximum $10(\alpha=.71)$.

Demographics measured were gender, age and education level which was categorized as low, medium or high level of education.

Use of the iailored intervention. To check the extent to which a primary condition for an effect was met, participants were asked at T2: "To what extent did you read the letter?" ("did not read it" (1) to "read it all" (10)).

\section{Statistical analyses}

Because two of the three outcome measures were binary, multivariate testing using the MANOVA procedure was not possible. For the binary measures (stage transition, seven days quit) logistic regression was used, and for the quantitative outcome measure (intention to quit) linear regression was. Sex, age and level of education were routinely included as covariates in the analyses to increase power by reducing unexplained variance (Robinson \& Jewell, 1991). The factor Condition was dumny-coded. All tests were 2tailed and $\alpha=05$ was used.

First, it was tested whether the tailored intervention had any effect in comparison with the control condition in the group of smokers as a whole. Second, the analyses were stratified according to Stage. Within each stage, each of the experimental conditions was compared to the no-information control group. It was expected that in each stage, only the hypothesized stagematched condition would have more effect than the other conditions. Next, the experimental conditions were compared to each other. A match-mismatch test was conducted to test whether smokers who received stage-matched information made more stage transitions, quit more often for seven days and had a higher intention compared to smokers who received information which was mismatched to their stage. The expectations, as explained in the introduction section, are depicted in Table 6.1. In a manipulation check it was assessed to what extent the conditions led to cognitive changes. Finally, it was checked to what extent the smokers had read the intervention materials. 
Table 6.1

Hypotheses on the stage matched and stage mismatched information in four motivational stages.

\begin{tabular}{lccl}
\hline & $N$ & $\begin{array}{l}\text { Matched } \\
\text { conditions }(\mathrm{N})\end{array}$ & $\begin{array}{l}\text { Mismatched } \\
\text { conditions }\end{array}$ \\
\hline Immotives & 310 & $\mathrm{OC}(107)$ & $\mathrm{BO}, \mathrm{SE}$ \\
Precontemplators & 226 & $\mathrm{BO}(63)$ & $\mathrm{OC}, \mathrm{SE}$ \\
Contemplators & 197 & $\mathrm{BO}(64)$ & $\mathrm{SE}, \mathrm{OC}$ \\
Preparers & 116 & $\mathrm{SE}(37)$ & $\mathrm{BO}, \mathrm{OC}$ \\
\hline
\end{tabular}

\section{Results}

\section{Participant characteristics, randomization check and attrition}

Of the 1540 respondents, $59 \%$ were female, the average age was 39.7 $(\mathrm{SD}=12.2)$, and regarding level of education, $22 \%$ were classified as "low", $43 \%$ as 'medium' and $35 \%$ as 'high'. The respondents smoked an average of 20.3 cigarettes a day ( $S D=9.1)$ and they had smoked for an average of 21.8 years $(S D=11.8)$. The average FTND score was $4.6(S D=2.5)$. Of the respondents, $44.9 \%$ had engaged in a serious quit attempt in the last welve months and $32.6 \%$ were categorized as immotives, $26.6 \%$ as precontemplators, $24.2 \%$ as contemplators and $16.6 \%$ as preparers. On the cognitive measures, the mean scores on the pros and cons of quitting were $1.59(\mathrm{SD}=0.6)$ and 1.26 $(S D=0.6)$ respectively. Self-efficacy with regard to emotional states and social situations was $-0.97(\mathrm{SD}=1.3)$ and $-0.19(\mathrm{SD}=1.2)$ respectively. Finally, the mean intention to quit was $5.9(\mathrm{SD}=2.8)$.

To check whether the randomization was successful, the four conditions were compared on sex, age, level of education, stage, FTND score, number of cigarettes a day, number of years smoked, quit attempts in the last 12 months, the pros and cons of quitting, both self-efficacy factors and intention to quit as measured at T1. As an additional check. the four conditions were compared on scores on the seven quitting plans. Chi-square tests for the discrete variables and F-tests for the contimuous variables revealed no significant differences between the conditions on any of these variables.

Attrition from pre-test (Tl) to post-test 2 (T3) was $28.8 \% \quad(n=440)$. Logistic regression analysis with attrition as dependent variable and the $T 1$ variables involved in the randomization check as predictors, revealed significant relations $(\mathrm{p}<05)$ : dropouts were more often male, dropped out more frequently from the $\mathrm{CO}$ condition than from the $\mathrm{OC}$ and the $\mathrm{BO}$ conditions and were more often contemplators or smokers in preparation. Table 6.2 gives the percentages of dropouts by Stage and Condition. Because attrition was related to Condition and to pre-test Stage, the analyses without the dropouts might lead to biased results. As a check on such bias, an additional 
analysis was run in which dropouts were included and in which their missing value (T3) was replaced by their last recording ( $\mathrm{T} 1$ or $\mathrm{T} 2$ ),

Table 6.2

Percentages of dropouts from TI to T3 per Condition and Stage.

\begin{tabular}{|c|c|c|c|c|c|c|c|}
\hline \multirow[b]{2}{*}{ Condition } & \multirow[b]{2}{*}{$\mathrm{N}$ at $\mathrm{T} 1$} & \multirow[b]{2}{*}{$\begin{array}{l}\text { Overall } \\
1540\end{array}$} & \multicolumn{5}{|c|}{ Stage of Change } \\
\hline & & & $\begin{array}{l}\text { Im } \\
502\end{array}$ & $\begin{array}{l}\mathrm{Pc} \\
409\end{array}$ & $\begin{array}{l}C \\
374\end{array}$ & $\begin{array}{l}\operatorname{Pr} \\
255\end{array}$ & $N$ at $T 3$ \\
\hline Overall & 1540 & 28.7 & 20.1 & 26.9 & 33.5 & 40.6 & 1100 \\
\hline Outcome & 384 & 23.4 & 19.5 & 20.8 & 29.4 & 26.4 & 295 \\
\hline Self-eff. & 385 & 29.6 & 25.9 & 21.6 & 31.5 & 46.4 & 272 \\
\hline Both & 386 & 26.4 & 12.7 & 33 & 30.4 & 39.4 & 284 \\
\hline Control & 385 & 34.8 & 23.5 & 32.4 & 43.3 & 47.1 & 251 \\
\hline $\mathrm{N}$ at $\mathrm{T3}$ & & 1100 & 401 & 299 & 248 & 152 & \\
\hline
\end{tabular}

Note. Im=immotives; $\mathrm{Pc}=$ precontemplators; $\mathrm{C}=$ contemplators; $\mathrm{Pr}=$ preparers

\section{Overall analyses}

We first tested whether the tailored intervention had any effect in comparison with the control condition in the group of smokers as a whole. The analyses showed that the experimental conditions led to significant $(p<.05)$ increases compared to the control condition on all three outcome measures, with one exception. Offering outcome information only did not lead to more seven-day quitting than offering no information. In contrast analyses, none of the experimental conditions led to significantly better results than any of the other experimental conditions. Tables 6.3 and 6.4 show effects of Condition, overal] and per Stage.

\section{Contrasts}

Immorives. It was hypothesized that immotives would benelit most from the $O C$ condition. Concerning stage transition and the intention to quit, the conditions containing outcome information (OC and $\mathrm{BO}$ condition) led to significant increases compared to the control group. With regard to seven-day quitting, none of the experimental conditions led to significantly more quitting than the control group. In contrast analyses, the experimental conditions did not differ significantly from each other.

Precontemplarors. It was hypothesized that precontemplators would benefit most from the BO condition. Concerning stage transition, both conditions containing self-efficacy enhancing information (SE and $\mathrm{BO}$ condition) led 
significantly more often to a stage transition than the control group. All three experimental conditions led to significantly higher intention than the control condition. With regard to "refraining from smoking for seven days", none of the experimental conditions led to significantly more quitting than the control group. In contrast analyses, the experimental conditions did not significantly differ from each other.

Contemplators. It was hypothesized that contemplators, just like precontemplators, would benefit most from the $\mathrm{BO}$ condition. Concerning stage transition and the intention to quit, the experimental condition containing both sorts of information (BO condition) led to significant increases compared to the control group. With regard to "refraining from smoking for seven days", none of the experimental conditions led to significantly more quitting than the control group. In contrast analyses, only one contrast was significant $(\mathrm{p}<.05)$ : contemplators in the $\mathrm{BO}$ condition reported a higher intention than contemplators in the SE condition.

Preparers. It was hypothesized that preparers would benefit most from the SE condition. The condition containing self-efficacy enhancing information only (SE condition) led to significantly more stage transitions and significantly more seven-day quitting, than the control group. None of the experimental conditions led to a significantly higher intention to quit than the control group. In contrast analyses, only one contrast was significant $(p<.05)$ : preparers in the SE condition reported more seven-day quitting than preparers in the $O C$ condition.

\section{Table 6:3}

Intention to quit at T3 by Stage and Condition: means and raw betas adfusted for Sex. Age and Educurion.

\begin{tabular}{llllllll}
\hline & OC & OC vs CO & SE & SE vs CO & BO & BO vs CO & CO \\
\cline { 2 - 8 } & $\mathrm{M}$ & $\mathrm{B}$ & $\mathrm{M}$ & $\mathrm{B}$ & $\mathrm{M}$ & $\mathrm{B}$ & $\mathrm{M}$ \\
\hline Overall & 5.89 & $.61^{* * *}$ & 5.82 & $.60^{* * *}$ & 6.18 & $.89^{* * *}$ & 5.27 \\
Immotives & 3.80 & $.74^{*}$ & 3.61 & .53 & 3.94 & $.88^{* *}$ & 3.04 \\
Preconternplators & 6.38 & $.73^{*}$ & 6.66 & $1.0^{* * *}$ & 6.79 & $1.14^{* * *}$ & 5.66 \\
Contemplators & 7.92 & .54 & 7.57 & .18 & $8.22^{*}$ & $.84^{* *}$ & 7.38 \\
Preparcers & 7.60 & .18 & 7.55 & .12 & 7.98 & .61 & 7.40 \\
\hline
\end{tabular}

Note. Participants who at T3 stated they had refrained from smoking for seven days were excluded from the outcome analysis of intention to quit smoking. Condition was dummy coded. A positive beta means that the effect in the experimental group exceds that of the control group (last columm). The betas and means are adjusted for Sex, Age and Education. "Contrasts analyses between the three expermental conditions were computed within ach stage: only contemplators in the $\mathrm{BO}$ condition had a significantly higher intention to quit than contemplators in the SE condition. *p<.05.**p<01.***p<.001. OC=outcome information; SE-wellefficacy enhancing information; BO=both sorts of information; $\mathrm{CO}=$ no information. 
Table 6.4

Siage trawsition and point prevalence abstinence (sever days awid as 73 by stage and Condition percentages and odds ratios.

\begin{tabular}{|c|c|c|c|c|c|c|c|}
\hline & $O C$ & $\mathrm{OC}$ vs $\mathrm{CO}$ & $S E$ & SE vSCO & $\mathrm{BO}$ & $\mathbb{B O}$ vs CO & $\mathrm{CO}$ \\
\hline & $\%$ & OR & $\%$ & OR & $\%$ & OR & $\%$ \\
\hline \multicolumn{8}{|c|}{$\begin{array}{l}\text { Forward transition } \\
\text { (backward transition) }\end{array}$} \\
\hline Overall & $\begin{array}{c}31.2 \\
(11.5)\end{array}$ & $1.89 *$ & $\begin{array}{c}36.5 \\
(10.2)\end{array}$ & $2.42 * *$ & $\begin{array}{l}35.6 \\
(8.4)\end{array}$ & $2.344 * 3$ & $\begin{array}{l}19.1 \\
(17.4)\end{array}$ \\
\hline Immotives & 32 & $2.11 *$ & 27.5 & 1.85 & 30.6 & $2.09 *$ & 17,4 \\
\hline $\begin{array}{l}\text { Precontem- } \\
\text { plators }\end{array}$ & $\begin{array}{l}28.8 \\
(5.5)\end{array}$ & 1.9 & $\begin{array}{l}40.7 \\
(4.9)\end{array}$ & $3.23 *$ & $\begin{array}{l}34.5 \\
(1.7)\end{array}$ & $2.50^{*}$ & $\begin{array}{l}17.1 \\
(15.7)\end{array}$ \\
\hline $\begin{array}{l}\text { Contempla- } \\
\text { tors }\end{array}$ & $\begin{array}{l}29 \\
(14.5)\end{array}$ & 1.71 & $\begin{array}{l}32.2 \\
(18.6)\end{array}$ & 1.91 & $\begin{array}{c}39.3 \\
(16.4)\end{array}$ & $2.64 *$ & $\begin{array}{l}20.0 \\
(28.9)\end{array}$ \\
\hline Preparers & $\begin{array}{r}37.8 \\
(48.6) \\
\end{array}$ & 1.70 & $\begin{array}{r}54.3 \\
(31.4) \\
\end{array}$ & $3.49 \%$ & $\begin{array}{r}45.9 \\
(30.6) \\
\end{array}$ & 2.60 & $\begin{array}{r}25.7 \\
(48.6)\end{array}$ \\
\hline \multicolumn{8}{|c|}{ Seven days quit } \\
\hline Overall & 4.8 & 1.52 & 8.5 & $2.91^{*}$ & 8.1 & $2.7 I^{*}$ & 3.2 \\
\hline Immotives & 1.9 & - & 1.2 & - & 3.4 & - & 1.1 \\
\hline $\begin{array}{l}\text { Precontern- } \\
\text { plators }\end{array}$ & 5.3 & 2.03 & 7.0 & 2.73 & 4.8 & - & 2.7 \\
\hline $\begin{array}{l}\text { Contempla- } \\
\text { tots }\end{array}$ & 6.9 & 1.84 & 8.2 & 2.11 & 10.9 & 2.94 & 3.9 \\
\hline Preparers & 7.7 & - & $29.7^{3}$ & $5.00 *$ & 22.0 & 2.81 & 8.3 \\
\hline
\end{tabular}

Note. Odds ratios with $p>5$ are not depicted. Significant odds ratio $>1.0$ means that the percentage in the experimental condition is significantly higher than the percentage in the control condition (last column). The odds ratios (but not the percentages) are adjusted for Sex, Age and Education. "Contrasts analyses between the three experimental conditions were computed within each stage: only preparers in the SE condition had quit significantly more often for seven days than preparers in the oC condition. ${ }^{*} p<05$. * $p<01$. * * $\mathrm{p}<001$. OC=outcome information; $\mathrm{SE}=$ self-eflicacy information; $\mathrm{BO}=$ both sorts of information; CO=no information.

\section{Match-mismatch test}

Smokers who were assigned to a stage-matched condition were compared to smokers who were assigned to a stage-mismatched condition on all three outcome measures. Table 6.1 shows that the sizes of three of the four matched groups were rather small for tests using binary outcome variables. Therefore, the analyses in smokers with low readiness to quit (immotives and precontemplators) were combined. That is, matched smokers (immotives in the $O C$ condition and precontemplators in the $B O$ condition) were compared 
to mismatched smokers (immotives in the $\mathrm{BO}$ and $\mathrm{SE}$ condition and precontemplators in the $\mathrm{OC}$ and $\mathrm{SE}$ condition). A binary match-mismatch variable was computed and entered in regression analyses for each of the three outcome measures. The same was done for smokers with high readiness to quit. That is, contemplators in the $\mathrm{BO}$ condition and preparers in the SE condition (matched) were compared to contemplators in the $\mathrm{OC}$ and $\mathrm{SE}$ condition and preparers in the $\mathrm{OC}$ and $\mathrm{BO}$ condition (mismatched). The match-mismatch tests showed the following:

In smokers with low readiness to quit who received stage-matched information, the percentage stage transition did not differ significantly from those who received mismatched information $(32.9 \%$ versus $31.9 \%)$. Furthermore, nonsiginificant differences were found with regard to seven-day quit $(2.9 \%$ versus $4.1 \%)$ and the intention to quit ( 5.10 versus 4.94$)$.

In smokers with high readiness to quit who received stage-matched information, the percentage stage transition was borderline significantly $(p<.10)$ higher than for those who received mismatched information $(44.8 \%$ versus $34.7 \%$ ). With regard to seven-day quit, matched smokers had quit borderline significantly more than mismatched smokers $(17.8 \%$ versus $10.3 \%)$. The intention to quit did not differ significantly between smokers who were and were not matched (7.80 versus 7.99).

\section{Post-hoc analyses}

The first set of post-hoc analyses concerned the distinction between immotives and precontemplators. Because the match-mismatch tests in smokers with low readiness to quit were nonsignificant, it might be argued that the present distinction between immotive and precontemplating smokers was not justified in the first place. Therefore, post-hoc analyses were computed in which immotive and precontemplating smokers were pooled in one group of smokers who were not planning to quit within the following six months. In contrast analyses it was tested whether this group as a whole benefitted more from one experimental condition than from another. Furthermore, three possible match-mismatch analyses were computed on the data from this pooled group: 1) considering the $O C$ condition to be matched and both other conditions mismatched; 2) considering the $\mathrm{BO}$ condition to be matched and both other conditions mismatched; 3) considering the SE condition to be matched and both other conditions mismatched. None of the contrast analyses and none of the match-mismatch analyses revealed significant effects with regard to stage transition, seven-day quit and intention to quit. Hence, compared to the pooled group, the data neither refuted nor justified the distinction between immotives and precontemplators.

A second set of post-hoc analyses concerned the data on backward stage transition, as depicted between brackets in Table 6.4. This was done because positive effects of the interventions might be neutralized by backward stage transition. In immotives, no backward ransition is possible. Furthermore, 
from all possible contrasts between the four conditions, only in contemplators the three experimental conditions led to significantly fewer backward transition than the control group. The match-mismatch analyses, hypothesizing that fewer matched smokers would make a backward transition than mismatched smokers, revealed no differences.

\section{Manipulation check and reading the letter}

It was expected that: 1) an intervention containing outcome information would cause an increase in expected positive outcomes and; 2) an intervention containing self-efficacy enhancing information would cause an increase in self-efficacy; 3) both sorts of information were expected to cause a decrease in the expected negative outcomes (Dijkstra et al., in press-a). Table 6.5 shows the means of all four cognitive measures per Condition. Participants who had refrained from smoking for seven days were excluded from the analyses leaving 1032 participants: 393 immotives; 284 precontemplators; 229 contemplators; 126 preparers. Outcome information only (OC) as well as both sorts of information (BO) led to the anticipation of more pros than no information ( $\mathrm{CO}$ ). A contrast analysis revealed that outcome information only led to the anticipation of significantly more pros than the combination of information. Self-efficacy enhancing information only (SE) led to an increase in self-efficacy to cope with social situations. No significant effects were found with regard to the cons of quitting and selfefficacy in coping with emotions. Concerning the extent to which smokers reported having read the tailored letter, there were no effects of Stage or Condition. The mean scores, on a 10-point scale, all ranged from 9.27 to 9.85 .

Table 6.5

Manipulation check: Means and raw betas of four cognitive variables at 13 per Condition, adjusted for the pretest score, Sex, Age and Education.

\begin{tabular}{lccccccc}
\hline & OC & OC vs CO & SE & SE vs CO & $B O$ & $B O v s C O$ & CO \\
\hline variable & $M$ & $B$ & $M$ & $B$ & $M$ & $B$ & $M$ \\
\hline Pros & 1.72 & $0.16^{* *}$ & 1.58 & 0.02 & 1.64 & $0.08 *$ & 1.56 \\
Cons & 1.19 & -0.01 & 1.2 & 0.01 & 1.22 & 0.03 & 1.19 \\
SE-social & 0.00 & 0.14 & 0.06 & $0.19 *$ & -0.05 & 0.09 & -0.013 \\
SE-emotional & -0.74 & 0.16 & -0.77 & 0.13 & -0.81 & 0.08 & -0.89 \\
\hline
\end{tabular}

Note Participants who at $T 3$ stated they had refrained from smoking for seven days were excluded from these analyses. Condition was dumny coded. A positive beta means that the mean score in the experimental group exceeds that of the control group. "p 0.05 . $* * p<01 . * * 0<001 . \quad O C=0$ outcome information $(N=281)$ : SE=self-efficacy enhancing information $(\mathrm{N}=249) ; \mathrm{BO}=$ both sorts of information $(\mathrm{N}=261) ; \mathrm{CO}=\mathrm{no}$ information $(N=243)$. 


\section{Analyses including dropouts}

Because attrition at $\mathrm{T} 3$ could be predicted by Condition, Sex and Stage at $\mathrm{T} 1$, the present results might be influenced by selective drop-out. One way to study this problem is to use the last known measurement (TI or T2) of each dropout as substitute for the T3 measurement and repeat all analyses (Heyting, Tolboom \& Essers, 1992). Data of 440 participants were missing at T3 because they dropped out. Of these missing data, 145 were replaced by T2 data and 295 by $\mathrm{Tl}$ data. With regard to the seven days quit outcome measure, all drop-outs were considered to have smoked for the last seven days. These analyses revealed that none of the significant results changed qualitatively. Only minor changes in betas, odds ratios and p-values emerged.

\section{Discussion}

The first goal of this study was to assess what sort of information smokers in each stage have to be offered in smoking cessation interventions. A test of mainly practical value was to compare each of the three experimental conditions with the no information control condition. Considering stage transition the outcome measure that was appropriate to all stages, the following results were obtained. In immotives, both conditions containing outcome information led to more transitions than the control condition. In precontemplators, both conditions containing self-efficacy enhancing information led to more transitions than the control condition. In contemplators, the condition containing both sorts of information led to more transitions than the control condition, and in preparers, the condition containing self-efficacy enhancing information only led to more transitions than the control condition. The hypotheses regarding contemplators and preparers were verified, but in immotives and precontemplators this was only partly the case. Moreover, none of the contrasts between the experimental groups, with regard to stage transition, were significant. Hence, these results can only lead to preliminary recommendations for interventions directed at each stage. Practically, two interventions seem to be needed; one intervention containing outcome information and self-efficacy enhancing information to be directed at immotives, precontemplators and contemplators, and one intervention containing self-efficacy enhancing information only, directed at preparers.

The second goal of this study was to assess whether the principle of stagematched information could be demonstrated. The match-mismatch tests revealed that smokers with high readiness to quit who were assigned to the matched treatment made borderline significantly more stage transitions and quitted borderline significantly more often than smokers who were assigned to a mismatched treatment. In addition, the contrast analyses revealed that: 1) contemplators in the condition offering both sorts of information had a higher intention to quit than contemplators in the condition offering self-efficacy enhancing information only; 2) preparers in the condition offering self- 
efficacy enhancing information only had quit more often for seven days than preparers in the condition offering outcome information only. The combination of the results of the comparisons with the no-information control group, the results from the contrast analyses and those from the match-mismatch analyses, strongly indicate that contemplators need a combination of outcome information and self-efficacy enhancing information and preparers need selfefficacy enhancing information only. In other words, contemplators seem to benefit from a different intervention than preparers. Together with the findings of earlier research that the effectiveness of smoking cessation interventions is lower for smokers in the contemplation stage than for smokers in the preparation stage (Prochaska, 1991; DiClemente et al., 1991), this strongly supports the distinction between both stages of high readiness to quit.

Concerning smokers with low readiness to quit, the results were partly unexpected. Smokers who were assigned to a matched treatment did not benefit more from treatment than smokers who were assigned to a mismatched treatment. This could mean that it does not really matter what kind of information smokers with low readiness are offered, as long as they are offered information on smoking and smoking cessation. Especially in immotives, the conditions led to only small differences in percentages of stage transition: whereas the largest difference between the three experimental conditions in immotives was $4.5 \%$, the largest difference in precontemplators was $11.9 \%$, in contemplators $10.3 \%$ and in preparers $16.5 \%$. In precontemplators, the data on the intention to quit showed that all three experimental conditions led to a significant increase compared to the control condition. These data on stage transition in immotives and intention to quit in precontemplators seem to support the notion that none of the presently used sorts of information were specifically matched or mismatched in smokers with low readiness to quit. The following explanations might be considered. First, the lack of matched or mismatched information, could be related to the existence of smoker subiypes (Velicer, Hughes, Fava, Prochaska \& DiClemente, 1995). For example, some smokers with low readiness to quit, including those in the groups of immotives and precontemplators, might benefit more from outcome information whereas others might benelit more from self-efficacy enhancing information. Second, it may be that the currently used information conditions did not manipulate matched or mismatched information which was relevant for smokers with low readiness to quit. For example, it might be that in these smokers other variables such as framing of the information or the length of the intervention messages can be matched or mismatched. Third, it might be that in smokers with low readiness to quit, messages on smoking and smoking cessation do not, at this stage, change the targeted cognitions because they process little information on smoking and smoking cessation (DiClemente et al., 1991; Prochaska et al., 1992; Prochaska, Velicer, DiClemente \& Fava, 1988). The fact that the taillored interventions did, however, lead to more changes than no intervention, might be 
understood by regarding the tailored interventions as a "cue to action". Although these explanations may be plausible, the present findings did not support the hypothesized matching for immotives and precontemplators. In that respect, smokers in contemplation and preparation differ from smokers with low readiness to quit, thereby supporting the distinction between smokers with high readiness to quit and those with low readiness to quit.

The data revealed some additional relevant results. For example, the results obtained with the primary outcome measure stage transition, were supported by the more conventional outcome measures: intention to quit revealed about the same pattern of effects in immotives, precontemplators and contemplators and the seven-day quit measure revealed the same pattern in preparers. In fact, the stage transition outcome measure combined both conventional outcome measures: the early stages are defined in terms similar to a measure of intention, whereas the later stage (i.e. the action stage) is defined in terms of behavior.

The effects of the tailored intervention are considered to be mediated by changes in the cognitions, that is, expected outcomes and self-efficacy expectations. The basic condition for these cognitive changes seemed to have been met: in all three experimental conditions, smokers in the four stages reported having read the letter very well. The average scores on a 10-point scale were all above 9 . This high figure might be explained by the personal relevance of the tailored letters: smokers recognized their own name, smoking behavior and psychological profile in the intervention.

The expected changes in cognitions could partly be detected. Information on the outcomes of quitting only, led to the anticipation of more pros of quitting, whereas self-efficacy enhancing information only, led to more confidence in the ability to cope with social situations. Offering both sorts of information at the same time resulted in the anticipation of more pros of quitting. The effects were small and the anticipation of the cons of quitting and the perceived self-efficacy in coping with emotions was not significantly changed by the interventions. However, the results on the cognitive changes might be underestimated by the exclusion of the seven-day quitters from these analyses. It is promising that, despite this exclusion, five to seven pages of tailored information were able to change relevant cognitions after ten weeks.

One limitation of the present study is the short follow-up. It could be that changes in smokers in the different stages meed more time to develop or, the other way around, that the detected changes will decline over time ${ }^{2}$. In

\footnotetext{
"At the the this article was submitted, a not planned fourteen months follow-up measurement was conducted. The results can be summarized as follows. The owerall analyses showed that none of the compra sons between the lour intervention groups were significant with regard to stage transition and sewen day cluttitng. With regard to intention to quit, only the $O C$ condition and the BO condition led to a significantly higher score compared to no information. Furthernore, neither the strafifed contrast andyses nor the matchmismatch analyses showed significant resulis. However, the fourteen months follow-up data showed that all three intervention conditions did lead to significanly more 24 -hour quid atempts durng the previous twelve months and that the the BO condition did lead to significantly more continuous abstinence - compared to the no infomation control condition.
} 
addition, we do not know to what extent the drop-out influenced the results, although the 'intention-to-treat' analyses suggest that this difference was small. Furthermore, the sample is still selective: Immotives and precontemplators with probably relatively little psychological defense for the confrontation with their smoking behavior joined the study. On the other hand, it seems an appropriate sample to draw conclusions from, since future applications of tailored information can well be directed at these kinds of samples.

In future research, the present stage-matched results need to be replicated and the group of smokers with low readiness to quit should be especially addressed. There is a need for further mapping of this population of smokers. Furthermore, different match-mismatch manipulations could be developed for this group. The fact that the percentages of smokers with low readiness to quit comprise a significant proportion of the smoking population, both in the Netherlands (70\%: Mudde et al., 1994) and in the U.S. (40\%: Velicer, Fava et al., 1995), justifies continuous efforts to target this group. 


\title{
Chapter 7
}

\section{Long-term effectiveness of computer-generated tailored feedback in smoking cessation'.}

\begin{abstract}
Although taillored interventions consisting of only a few pages of information lead to more quitting than no intervention in the short term, the long-term efficacy of a single tailored intervention still has to be proven.

In the present study smokers were reactively recruited and randomly allocated to one of four intervention conditions: 1) Outcome information or: 2) Self-efficacy enhancing information or: 3) Both sorts of information or: 4) No information. Smokers in the three experimental groups received computer-generated tailored feedback containing the condition specific information, by mail.

The results from the fourteen months follow-up can be summarized as follows. Compared to the no information condition, all three experimental conditions led to significantly more smokers who had engaged in 24-hour quit attempt. However, no experimental condition led to more seven-day quitting. than the no information condition. With regard to continuous abstinence, the experimental condition offering a combination of outcome information and selfefficacy enhancing information had a significant effect, compared to the no information condition. It is concluded that a minimal 6-page tailored intervention can be beneficial in supporting smokers to quit smoking, even after fourteen months.
\end{abstract}

This chapter will be published as: Dijkstra, A., De Vries, H., \& Roijackers, J. (in press). Long-term effectiveness of computermenerated tailored feedback in smoking cessation. Heath Education Research. 


\section{Introduction}

Self-help materials in smoking cessation are designed to support smokers in their further unaided quit attempts (Cummings et al., 1988; Glynn, Boyd, \& Gruman, 1990; Lichtenstein \& Glasgow, 1992). However, most self-help materials are directed at smokers in general and not at individuals. Hence, these standardized self-help interventions contain information which is often not applicable to an individual's psychological state or situation. Tailoring information to individual features of a person has been shown to be a promising line of development in self-help interventions. It is considered to enhance the relevance of the offered information and to lead to larger behavioral effects (De Vries, Willemsen, Brug, Dijkstra \& Berben, 1995; Strecher et al. 1994; Velicer et al., 1993).

Because computer-generated tailored interventions mostly consist of only a few pages of information, decisions have to be made about which information is essential to behavior change (Dijkstra, De Vries \& Roijacker, in press-a). Cummings et al. (1988) propose that self-help interventions need to contain the following information: a) Information about the health and social consequences of smoking; b) Specific strategies and exercises for successful quitting; and c) Specific strategies and exercises for the maintenance of non-smoking and the prevention of and coping with relapse. From a social cognitive theoretical point of view (Bandura, 1986), this information is considered to change cognitive anticipation of the outcomes of quitting (information a) and to enhance perceived self-efficacy (information b and c) (Dijkstra et al., in press-a), which are two major determinants of behavior and behavior change (Bandura, 1986). Cummings et al. argue that the level of readiness to quit varies widely, even in smokers who seek advice on quitting and they recommend that future quit smoking self-help materials should include: "... information designed to increase motivation for quitting as well as suggestions on how to quit" (p. 215). In other words, a number of the smokers who show interest in smoking cessation have low readiness to quit and may benefit from outcome information. On the other hand, if the outcome information could be omitted from an intervention, other and more relevant information could be added. Such an innovation would be expected to enhance efficacy of the (tailored) intervention.

A primary test of the efficacy of self-hellp materials compares the effects with a no-intervention control group. Only when an intervention leads to more behavioral changes than no intervention, is it useful to distribute and implement it. A search of the literature reveals that a single tailored intervention letter can indeed lead to more changes in behavior than no information. However, this conclusion can only be drawn with regard to short-term elfectiveness (Campbell et al., 1994; Kreuter \& Strecher, 1996; Owen, Ewins \& Lee, 1989; Strecher et al., 1994).

The first goal of the present study was to assess whether computerized tailored letters comprising about 6 pages of information, led to more quitting than no intervention after 14 months. The second goal of the present study was to assess which type of information would be more successful: Information on 
outcomes of quitting only, self-efficacy enhancing information only or a combination of both sorts of information. Additionally, the cognitive characteristics associated with successful quitting were examined.

\section{Method}

\section{Participant recruitment}

Smokers were recruited by advertisements in local newspapers throughout the Netherlands. They were asked to volunteer for a research project on minimal interventions for smoking cessation, including the possibility of being a control group member. In order to assure a high variation of readiness to quit in the sample, smokers without plans to quit were also explicitly invited to react. Participants were offered the opportunity of 10 bonus prizes amounting to $\$ 100$. After participants had phoned the university in order to register $(n=1733)$, they were sent the pre-test questionnaire which could be returned with a pre-paid envelope. After two weeks, 1546 pre-test questionnaires $(89.2 \%)$ had been returned (excluding sixty questionnaires of pipe or cigar smokers and questionnaires filled out incompletely).

\section{Design}

Smokers were randomly assigned to one of four conditions: Only information on outcomes of quitting (OC condition, $n=386$ ), only self-efficacy enhancing information ( $\mathrm{SE}$ condition, $\mathrm{n}=387$ ), both sorts of information (BO condition, $\mathrm{n}=387$ ) or no information at all (CO condition, $\mathrm{n}=386$ ). Participants in the experimental conditions were sent the intervention material by mail, while participants in the control condition received a letter in which they were told they would get no information.

The present article presents the data of $T 1$, the first post test ( $T 2$; two weeks after the intervention) and the 14 months follow-up (T3). The data on the three months follow-up are published elsewhere (Dijkstra, De Vries, Roijackers \& Van Breukelen, in press-d) because specific short-term outcome measures were used. The tailored interventions, also, are described in more detail in that study.

Participants at $\mathrm{T} 1(\mathrm{~N}=1546)$ were (14 months after the intervention) sent a short questionnaire which started with the announcement of a $\mathrm{CO}$-validation measurement in a sample of the participants who reported having quit smoking. This announcement was considered to act as a bogus pipeline (Jones \& Sigal, 1971). Participants who returned the 14 months follow-up questionnaire were offered the opportunity of 20 bonus prizes amounting to $\$ 50$. From the 1546 questionnaires sent, $990(64 \%)$ were returned.

\section{Questionnaire}

Three outcome measures were used at the 14 months follow-up. First, quit 
activity was measured by asking: "Have you engaged in a 24-hour quit attempt since the last measurement" (yes/no). Second, point prevalence quitting was assessed by asking: "Have you smoked during the last seven days? (even one puff)" (yes/no). Third, continuous abstinence was assessed by asking: "Have you smoked since the last measurement?" (yes/no). The time between the last measurement and the 14 months follow-up was approximately 12 months.

The pre-test questionnaire assessed gender, age, level of education, the number of cigarettes smoked, the number of years participants had smoked, a 24 hour quit attempt in the last 12 months and the participants' nicotine dependence. The nicotine dependence was assessed with the Fagerström Test for Nicotine Dependence (FTND; Heatherton, Kozlowski, Frecker \& Fagerström, 1991). Furthermore, perceived self-efficacy, intention to quit and the pros and cons of quitting were assessed. The pros-of-quitting scale was composed of 15 items referring to the positive consequences of behavior change. Cronbachs $\alpha$ was .87 . The cons-of-quitting scale was composed of six items referring to the negative consequences of behavior change $(\alpha=57)$. The scales had a significant but low correlation $(r=24)$ and were validated in an earlier study (Dijkstra, De Vries \& Bakker, 1996). The items of both scales could be scored from "not sure" or "not expecting a certain outcome" $(0)$ to a "strong expectation of the outcome" (3).

Two self-efficacy scales were used, assessing self-efficacy with regard to coping with social situations ( 4 items, $\alpha=81$ ) and self-efficacy with regard to coping with negative emotions ( 3 items, $\alpha=86$ ). All self-efficacy expectations were measured on a 7-point scale and could be scored from "not sure at all whether I am able to refrain from smoking" (-3) to "very sure I am able to refrain from smoking" $(+3)$ and the item scores were added to scale scores.

The questionnaire at $T 2$ contained six process evaluation items. Three items assessed to what extent the participants in the experimental groups experienced the interventions as containing information on: 1) the pros of quitting; 2) the cons of quitting; 3 ) skills to quit smoking. The remaining three items assessed to what extent participants in the experimental conditions: 1) perceived more pros of quitting; 2) perceived fewer cons of quitting; 3) learned skills to quit smoking, due to the tailored intervention. All six items could be scored from "not at all" (1) to "to a large extent" (10).

\section{Participant characteristics, randomization check and attrition}

Of the 1546 respondents, $59 \%$ were female, the average age was 39.7 and $22 \%$ were classified as low educated, $43 \%$ as medium educated and $35 \%$ as high educated. The respondents smoked an average of 20.3 cigarettes a day, they had smoked for an average of 21.8 years and $44.9 \%$ of the respondents had engaged in a serious quit attempt in the last twelve months. The average FTND-score (the scale ranging from $0-10$ ) was 4.6 . With regard to the cognitive measures, the mean scores on the pros and cons of quitting (the scale ranging from $0-3$ ) were 1.59 and 1.29 , respectively. Self-efficacy mean scores with regard to 
emotional states and social situations (the scale ranging from minus 3 to +3 ), were -0.97 and -0.19 , respectively. Finally the mean intention to quit (the scale ranging from $1-10$ ) was 5.9 .

To check whether the randomization was successful, the four conditions were compared on sex, age, level of education, FTND-score, number of cigarettes a day, number of years smoked, quit attempts in the last 12 months, the pros and cons of quiting, both self-efficacy factors and intention to quit as measured at T1. Chi-square tests for the discrete variables and F-tests for the continuous variables revealed no differences between the conditions with respect to these variables.

Attrition from pre-test (T1) to the post-lest at 14 months was $36 \%(n=555)$. Logistic regression analysis with attrition as the dependent variable and the T1variables involved in the randomization check as predictors, revealed that dropouts perceived significantly $(p<.05)$ fewer pros of quitting but had a significantly higher intention to quit.

\section{Analyses}

First, to check whether the interventions in the experimental conditions were experienced as containing the different sorts of information, outcome information or self-efficacy enhancing information, the group means on the process evaluation items were compared using ANOVAs, adjusted for sex, age and level of education. The same procedure was used with regard to the experienced change in perception of the pros, cons and self-efficacy. All participants in the experimental conditions who responded at $\mathrm{T} 2$ and at the 14-months follow-up were selected in these analyses.

Second, to test whether, and which of the experimental conditions still had an effect after 14 months, logistic regression analyses were used with 24 -hour quit attempt, seven-day quit and continuous abstinence as dependent variables and Condition as the independent variable. The analyses were adjusted for sex, age and level of education. The factor Condition was dummy coded, using the control condition as the reference group. In case the effect in two or three experimental conditions exceeded the effect in the control condition, contrasts. between the experimental conditions were computed.

Third, in order to examine the characteristics of smokers who had quit smoking, the pros and cons of quiting, both self-efficacy measures and the FTND scores at $\mathrm{T} 1$ were entered in a logistic regression to predict quitting after 14 months. The analyses were adjusted for sex, age, level of education and Condition.

\section{Results}

\section{Manipulation check}

Table 7.1 shows the mean scores on the six process evaluation items by 
Condition. In both conditions offering outcome information $10 \mathrm{C}$ and $\mathrm{BO}$ condition), participants scored higher on the recognition of information on the pros and the cons of quitting, than in the condition designed to not contain this information (SE condition). In both conditions offering self-efficacy enhancing information ( $\mathrm{SE}$ and $\mathrm{BO}$ condition), participants scored higher on the recognition of information on skills to quit smoking, than participants in the condition designed to not contain this information (OC condition). Also, participants in both conditions containing outcome information (OC and $\mathrm{BO}$ condition), stated that they perceived more pros of quitting after the intervention, than participants in the SE condition. No differences occurred with regard to changes in the perceived cons of quitting. Participants receiving self-efficacy enhancing information ( $\mathrm{SE}$ and $\mathrm{BO}$ condition) reported having learned more skills to quit smoking after the intervention, than participants in the $O C$ condition. Except with regard to (the lack of) change in the perception of the cons of quitting, all differences were in the expected directions.

\section{Table 7.1}

Means of the process evaluation items by experimental condition, adjusted for Sex, Age, Education and Stage of Change.

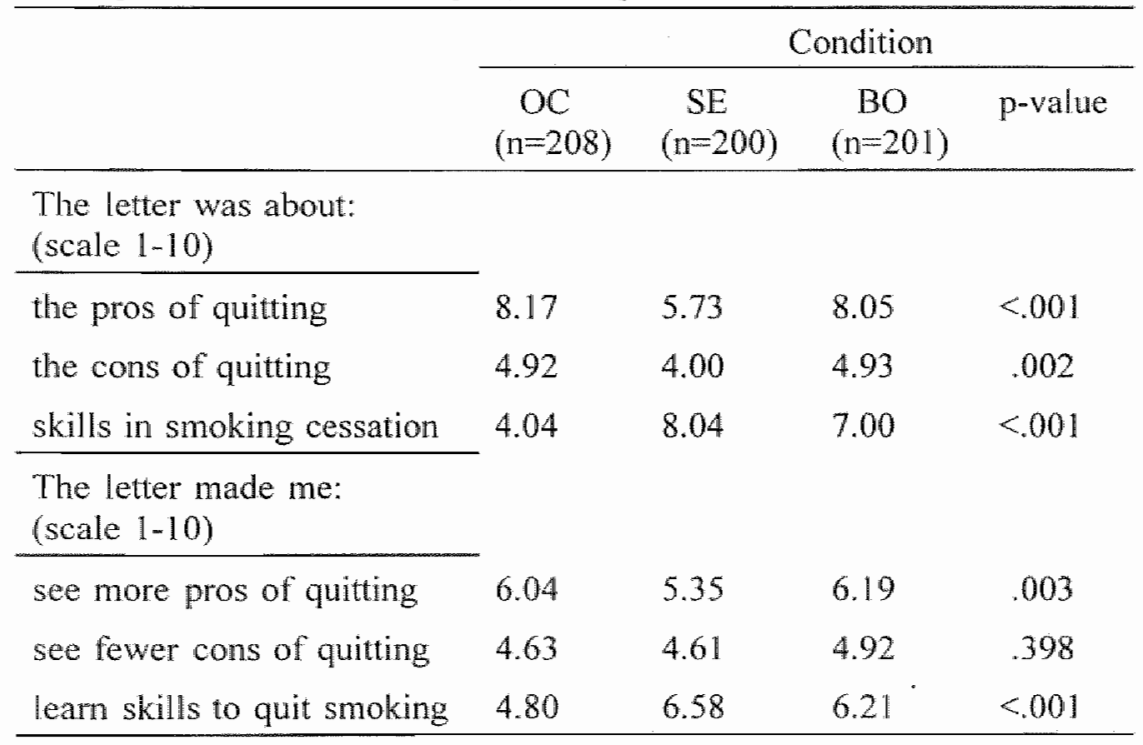

\section{Effects on smoking cessation}

Table 7.2 shows the percentage of quitters after 14 months by Condition and the odds ratios for the comparison with the control condition. With regard to engaging in a 24-hour quit attempt since the last measurement, all three experimental conditions led to significantly higher $(\mathrm{p}<.05)$ percentages of quit attempts than the no information control condition: About $50 \%$ of the smokers 
in the experimental conditions reported having engaged in a quit attempt of at least 24 hours since the last measurement, in comparison with $43 \%$ in the control condition. Contrast analysis revealed that the experimental conditions did not differ from each other. With regard to the seven-day quit criterion, no experimental condition led to more seven-day quitting than the control condition. In the control condition, but also in two of the experimental conditions, about $11 \%$ had quit for sever days. With regard to continuous abstinence, the experimental condition offering a combination of outcome information and selfefficacy enhaneing information ( $\mathrm{BO}$ condition), led to significantly more $(\mathrm{p}<.05)$ participants reporting continuous abstinence than the no-information control condition. In the $\mathrm{BO}$ condition almost $5 \%$ reported having refrained from smoking since the last measurement, in comparison with $1.6 \%$ in the control condition. Both of the other experimental conditions (OC and SE condition) did not lead to significantly more continuous abstinence in comparison with the control group.

Table 7.2

424 hour quit altempl since the last measurement, seven-day quit and continuows abstinence: Percentages and odds ratios (OR) adjusted for Sew. Age and Faducation.

\begin{tabular}{lccccccc}
\hline & OC & OC vs CO & SE & SE vS CO & BO & BO vs CO & CO \\
\hline & $\%$ & OR & $\%$ & OR & $\%$ & OR & $\%$ \\
\hline 24 -Hour & 49 & $1.50 *$ & 50.4 & $1.57^{*}$ & 51.4 & $1.63^{*}$ & 42.8 \\
Seven-day & 8.5 & 0.78 & 11.5 & 1.07 & 11.3 & 1.05 & 11.2 \\
Cont Abst & 2.4 & 1.77 & 3.3 & 2.25 & 4.8 & $3.74^{*}$ & 1.6 \\
\hline
\end{tabular}

Note. Significant odds ratio $>1.0$ means that the percentage in the experimental condition is significantly higher than the percentage in the control condition (last column) * $\mathrm{p}<05$. $O C=$ outcome information $(n=247)$; $S E=$ self-efficacy information $(n=244)$; $B O$-both sorts of information $(n=249)$; $C O=$ no information $(n=250)$.

\section{Characteristics of quitters}

Table 7.3 shows the characteristics of smokers at $T 1$ which are associated with quitting. Smokers who reported having been engaged in a quil attempt since the last measurement anticipated more pros of quitting, had higher self-efficacy with regard to coping with social situations and lower FTND-scores. Smokers who reported having refrained from smoking for seven days also anticipated more pros of quitting and had lower FTND scores at T1. Smokers who reported being continuously abstinent after 14 months, anticipated more pros of quitting and had higher self-efficacy with regard to coping with negative emotions at T1.

\section{Analyses including drop-outs}

Because attrition could be predicted by the perceived pros of quitting and the 
intention to quit at $\mathbb{T} 1$, the present results might be infuenced by selective dropout. One way to address this problem was to use the last known measurement (T1 or T2) of each dropped-out smoker as a substitute for the T3 measurement and repeat all analyses. This 'intention to treat analysis' (Heyting, Tolboom \& Essers, 1992) revealed that none of the significant results changed qualitatively. Only minor changes in betas, odds ratios and p-values emerged. The "intention to treat analyses' seem to support the present results.

\section{Table 7.3}

Predicting quitting behavior at 14 months from pre-test scores on four cognitive measures and the FTND, adjusted for Sex, Age. Education and Condition.

\begin{tabular}{|c|c|c|c|c|c|c|}
\hline \multirow[b]{3}{*}{ Variables } & \multicolumn{6}{|c|}{ Outcome measures } \\
\hline & \multicolumn{2}{|c|}{$\begin{array}{l}\text { Continuous } \\
\text { abstinence }\end{array}$} & \multicolumn{2}{|c|}{$\begin{array}{l}\text { Seven-day } \\
\text { quitting }\end{array}$} & \multicolumn{2}{|c|}{$\begin{array}{l}\text { 24-hour quit } \\
\text { attempt }\end{array}$} \\
\hline & OR & $\mathrm{SE}$ & OR & $\mathrm{SE}$ & OR & $\mathrm{SE}$ \\
\hline Pros of quitting & $1.06^{* *}$ & 0.02 & $1.03^{*}$ & 0.01 & $1.06^{* * * * * *}$ & 0.01 \\
\hline Cons of quitting & 0.95 & 0.06 & 0.99 & 0.03 & 1.02 & 0.02 \\
\hline Emo. self-efficacy & $1.16^{*}$ & 0.07 & 1.05 & 0.04 & 0.99 & 0.03 \\
\hline Soc. self-eficacy & 1.06 & 0.06 & 1.03 & 0.03 & $1.08 * * *$ & 0.02 \\
\hline FTND & 0.92 & 0.09 & $0.83 * * *$ & 0.05 & $0.89^{*}$ & 0.03 \\
\hline
\end{tabular}

Note. ${ }^{*} p<.05 ; * * p<.01 ; * * * p<001 . N=990$. A significant odds ratio (OR) larger than 1.0 , means that the variable has a positive relation with quitting, whereas a significant odds ratio smaller than 1.0 means that the variable has a negative relation with quitting. $\mathrm{SE}$ =standard error.

\section{Discussion}

All three sorts of tailored information led to more quit attempts of at least 24 hours in the previous twelve months in comparison with no intervention, whereas no effect was found with regard to the seven-day quit criterion. A sixpage talored intervertion containing a combination of outcome information and self-efficacy enhancing information led to more continuous abstinence than no intervention, even after fourteen months. Since continuous absinence is the most conservative and most desirable outcome of smoking cessation interventions, the present study suggests that a sample of reactively recruited smokers with varying readiness to quit, can benetit from a combination of information on the outcomes of quitting and self-efficacy enhancing information.

Although the results with regard to continuous abstinence are clear, questions arise with regard to both of the other outcome measures. With regard to the 24hour quit attempt measure, it was found that all three experimental conditions 
had more effect than the control condition. In other words, it did not seem to mater which information they were offered, as long as they received infomation on smoking cessation. However, it might be questioned whether encouraging smokers to engage in 24 -hour quit attempts is an appropriate smoking cessation strategy. Because the retrospective report of a quit attempt of at least 24 hours implies that the smoker has relapsed in the past, such a failed quit attempt might be regarded as detrimental. On the other hand, if a 24-hour quil attempt is regarded as a learning experience (Curry et al., 1995; Orleans, 1993 ), all three sorts of information tested in the present experiment would seem beneficial.

None of the experimental conditions led to more seven-day quitting than the control condition. The fact that continuous abstinence was stimulated by a tailored message but engaging in a seven-day quit attempt was not, might be explained in the following way. Smokers who reported being continuously abstinent, started their quitting only a short time after the intervention, whereas most smokers who had refrained from smoking for the previous seven days (smokers who were continuously abstinent were included in this measure) started this attempt later or had relapsed at an earlier attempt. The effect with regard to continuous abstinence might be brought about a short time after the intervention in smokers who have a decreased risk of relapse. This notion is supported by the finding that in the present study, smokers who were continuously abstinent had higher self-efficacy to cope with negative emotions at pre-test, while negative emotions are a major source of relapse (O'Connel \& Martin, 1987; Shiffman, Paty, Gnys, Kassel \& Hickcox, 1996; Swan, Ward \& Jack, 1996). Moreover, whereas being a more nicotine dependent smoker led to fewer seven-day quitting and fewer quit attempts since the last measurement, in the present sample, nicotine dependence did not influence the chances of becoming continuously abstinent. It may be the case that smokers with higher self-efficacy in coping with negative emotions, have enough resources to abstain in spite of negative emotions caused by withdrawal from nicotine. Smokers who engaged in quit attempts since the last measurements had, at pre-test, higher self-efficacy to cope with social situations. This might mean that a high self-efficacy to cope with social situations helps to initiate a quit attempt, but is not sufficient in preventing relapse.

Concluding, a six-page tailored intervention was effective after fourteen months. In the present sample with varying readiness to quit, an intervention containing outcome information as well as self-efficacy enhancing information was beneficial. The fact that smokers were able to identify the sort of intormation in the tailored intervention, as the manipulation check revealed, further supports the conclusion with regard to the sort of information smokers benefit from. Furthermore, quitting behavior was associated with the anticipation of many pros of quitting. High self-efficacy with regard to coping with negative emotions at pre-test was a cognitive characteristic of smokers who were continuously abstinent after 14 months.

The fact that the presently used minimal but tailored intervention was 
effective in supporting smokers to still refrain from smoking after fourteen months, is very promising. Future research might be directed at enhancing the effectiveness by further dismantling the content of smoking cessation interventions in order to decide which information is essential to behavior change in samples with differing readiness to change. 


\title{
Chapter 8
}

\section{Tailoring information to enhance quitting in smokers with low motivation to quit: Three basic efficacy questions"}

\begin{abstract}
Tailoring information to a person's individual features is a promising line of development in self-help interventions. Tailored interventions are thought to have higher efficacy than non-tailored interventions, and, in contrast to standardized interventions, they can be offered to individuals more than once. Furthermore, tailored interventions can be used in combination with existing self-help materials. In the present experiment, 752 smokers with explicit low intention to quit, were randomly assigned to one of five conditions: 1) multiple tailored letters with an additional self-help guide; 2) multiple tailored letters only; 3) a single tailored letter with an additional self-help guide; 4) a single tailored letter only; or 5) a non-tailored intervention. The follow-up assessment was four montlys after the intervention.

The results showed that the single tailored intervention without the additional self-help guide had no surplus value compared to a non-tailored look-alike intervention. The addition of a self-help guide to a tailored intervention was only useful in highly dependent smokers and multiple tailoring was clearly more effective than single tailoring. It is concluded that tailoring information holds promise but that in the further development of tailored interventions it is important to elucidate why and for whom certain tailored interventions are more effective.
\end{abstract}

'This chapter will be published as: Dijkstra, A., De Vries, H., Roijackers, J., Van Breukelen, $G_{n}$ (in presst. Tailoring information to enhance quibring in smokers with low motivation to quit: Three basic efficacy questions. Health Psychology. 
In the Netherlands, $70 \%$ percent of smokers are not planning to quit within the next six months (Mudde, Dolders \& De Vries, 1994). Although the percentage of these so-called precontemplators in the U.S. is about $40 \%$ (Velicer et al. 1995), it still comprises a significant proportion of the smoking population. Precontemplating smokers have no interest in standardized self-help materials which mostly are designed for smokers who are preparing a quit attempt. However, by tailoring the content of a smoking cessation intervention to a person"s individual features, such as attitudes and motivational stage, even this group of smokers might be encouraged to quit. Using individual data, complex intervention messages can be composed by a computerized tailoring system (De Vries, Willemsen, Brug, Dijkstra \& Berben, 1995; Strecher et al., 1994; Velicer et al., 1993). In the literature on conputer generated tailored interventions, three main issues seem to be of importance.

The first and fundamental question is whether tailored interventions have higher efficacy than non-tailored interventions. The wltimate test to assess whether this is the case, is to compare a tailored intervention to a non-tailored intervention with the same lay-out, length and presentation, and addressing the same topics. Only a few studies have addressed the problem as thoroughly as this. The results of these studies can be summarized as follows. Compared to a non-tailored intervention, a tailored intervention led to more forward stage movement, only in black and low-income women (Skinner, Strecher \& Hospers, 1994), to significantly more quitters, but only in a group of lighter smokers (Strecher et al. 1994), and to a lesser fat intake (especially in the group of 'risk consumers'), but not to larger changes in fruit and vegetable consumption (Brug, Steenhuis, Van Assema \& De Vries, 1996; also see Campbell et al., 1994). The results show that tailored information can lead to more changes in behavior than non-tailored information, but the effect may be limited to certain behaviors and subgroups.

The second question regarding tailoring is whether tailored interventions in combination with an additional self-help guide, have higher efficacy than tailored interventions without this addition. Literature search shows that two forms of tailored interventions are used. The first form of tailored interventions consists of only a few pages of information (Kreuter \& Strecher, 1996; Owen, Ewins \& Lee, 1992; Strecher et al., 1994; also see De Vries et al., 1995). The second form consists of a tailored intervention in combination with a complementary and non-tailored self-help manual (Burling et al., 1989; Curry, McBride, Grothaus, Louie \& Wagner, 1995; Curry, Wagner \& Grothaus, 1991; Prochaska, DiClemente, Velicer \& Rossi, 1993). The latter, more extensive intervention, could be more powerful. However, as far as we know, no previous research has investigated the effect of tailored information in combination with a standardized self-help manual, compared to only tailored information.

The third question is whether multiple tailored interventions have higher efficacy than a single tailored intervention. Offering multiple tailored 
interventions may provide additional strength to change behavior. For example, the tailored information can be updated each time and subsequent tailored messages offer the opportunity to give feedback about the direction and extent of changes in determinants of behavior and behavior itself. Brug, Glanz, Van Assema \& Kok (1996) compared the effects of giving tailored feedback twice to giving tailored feedback once, in a randomized field trial. Giving tailored feedback twice had significantly more effect on fat consumption and vegetable intake than giving it once, although this was not the case for fruit consumption.

The goal of the present study was to analyze whether tailored information had more effect than non-tailored information, whether the addition of a standardized self-help guide to a (complementary) tailored intervention had more effect than a tailored intervention alone, and whether multiple tailoring was more effective than single tailoring. The high percentage of smokers with low motivation to quit in the Netherlands warrants the development and testing of effective interventions for this group. Hence, in the present experiment only smokers who were not planning to quit within the next six months participated.

\section{Method}

\section{Recruitment}

Smokers were recruited by advertisements in local newspapers throughout the Netherlands, in which they were asked to volunteer for a research project on smoking and smoking cessation. Some specific measures were taken to recruit a sample of smokers with low intention to quit. First, in the advertisements the target group was defined as: "Smokers who are not planning to quit within the next six months, or may be planning to never quit". Second, to minimize a selective sampling, it was stated explicitly that smokers did not have to quit to join in the present study. Third, subjects completing all questionnaires were offered the opportunity to win 20 bonus prizes amounting to $\$ 100$.

After smokers had phoned the university in order to register $(n=925)$, they were sent the pre-test questionnaire which could be returned in a pre-paid envelope. After two weeks $795(86 \%)$ pre-test questionnaires had been returned. Forty-three of these questionnaires were excluded, because the respondents only smoked a pipe or cigars, or they did have plans to quit within the next six months. This resulted in 752 respondents at TI.

\section{Design}

Smokers were randomly assigned to one of five conditions receiving: 1) three times (multiple) a tailored letter with additional self-help guide (MTplus condition; $N=140$ ); 2) three times (multiple) a tailored letter only (MTonly condition; $N=156$ ); 3) a single tailored letter with additional self-help guide (STplus condition; $\mathrm{N}=157$ ); 4) a single tailored letter only (STonly condition; $\mathrm{N}=152$ ); 5) once a single non-tailored letter (NT condition; $\mathrm{N}=147$ ). Two weeks 
after the pre-test questionnaines (TI) were retumed, the perticipunts were sent the intervention material (in the MT conditions the first talored letter) by mail. Four months after the interwention, participants were sent the post-test questionnaire (T2) (see Figure 8.1).

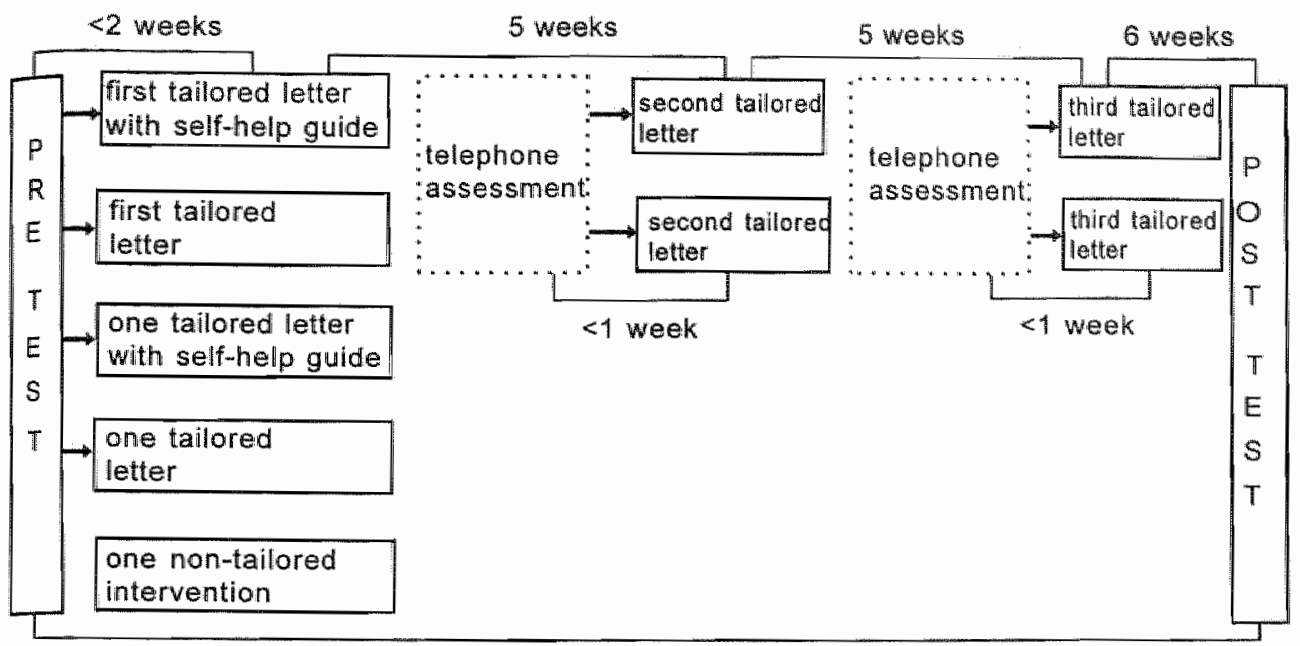

4 months

Figure 8.1 The design with five experimental conditions.

\section{Questionnaire}

Because the present sample had a low motivation to quit at the start of this study with short follow-up, it was expected that only a few smokers would quit smoking. Hence, behavioral outcome measures were regarded as less appropriate. Stage-transition and intention to quit were considered the primary outcome measures (see Velicer, Prochaska, Rossi \& Snow, 1992; Velicer, Rossi, Prochaska, DiClemente, 1996).

Stages of change were assessed by confronting smokers with different time plans with regard to smoking cessation. Four stages with increasing neadiness to quit were distinguished: immotivation, precontemplation, contemplation and preparation (Dijkstra, Bakker \& De Vries, 1997; Dijkstra, Roijackers \& De Vries, in press-c). Smokers who refrained from smoking for the last 24 hours were considered to be in the action stage.

Stage transition was assessed by dichotomizing changes in stage: Forward transition was scored as 1 , versus no transition or backward transition which was scored as 0 .

Intention to quit was measured with a composite of three 10-point scales: "Do you intend to quit smoking: 1) within the next six months; 2) within the next five years; 3) ever?" "The items could be scored from "not at all" (1) to "very much" (10). The composite intention score was the average item score (range 1- 


\section{0). Cronbach's reliability (o) was .83 .}

Furthermore, quitting behovior was used as an outcome measure. Quitting behavior was first measured by asking subjects whether they had engaged in a 24-hour quit attempt since the first measurement (yes/no). Second, a more conservative criterion was used to assess smoking cessation: "Have you smoked in the last seven days? (even one puff)" (yes/no).

To check whether the randomization procedure was successful with regard to some relevant determinants of quitting behavior, smokers in the five groups at TI were compared on scale scores on positive and negative outcome expectations, perceived self-efficacy, smoking behavior and demographics. The individual data were also used as the basis for the tailored interventions.

Thirty-three items, validated in earlier studies (Dijkstra et al., 1997; Dijkstra, De Vries \& Bakker, 1996) assessed the expected outcomes of quitting (Bandura, 1986). Expected positive outcomes were assessed with a 23 -item scale $(\alpha=90)$. Expected negative outcomes were assessed with a 10-item scale $(\alpha=82)$.

Thirteen items assessed self-efficacy expectations with regard to the ability to refrain from smoking in social and emotional situations $(\alpha=94)$.

Heaviness of smoking was measured by the Fagerström Test for Nicotine Dependence (Heatherton, Kozlowski, Frecker \& Fagerström, 1991) which assesses smoking habits: How much do you smoke? How soon after awaking? Is it hard not to smoke in public places? Do you smoke when you are ill? Do you smoke more in the morning? Which cigarette is most difficult to give up? (scale range $0-10 ; \alpha=71$ ).

Furthermore, smoking behavior was measured by asking smokers how many years they had been smoking, how many cigarettes they smoked on average and whether they had engaged in a 24-hour quit attempt in the last 12 months.

Demographics measured were gender, age and education level which was categorized as low, medium or high level of education.

All participants were asked to what extent they had read and remembered the (first) intervention message and to what extent they had learned from it. In the conditions with an additional self-help guide, participants were asked to what extent they had read the self-help guide and to what extent it had been useful. All these questions could be scored on a 10-point scale from "not at all" (1) to "to a large extent" (10).

No biochemical verification of the self-report quitting behavior was conducted, because first, it was expected that the announcement of a biochemical verification would increase non-response and drop-out, especially in the present sample with low motivation (see Velicer et al., 1992). Moreover, the present study was characterized by low demand, that is, at recruitment smokers were told they did not have to quit smoking; for this reason the self-report was considered to be valid (see Velicer et al., 1992).

\section{The tailored interventions}

General principles. The computerized system to generate the tailored letters was 
adapted from previous evaluations of minimal interventions in smokers who were planning (Dijkstra, De Vries \& Roijackers, in press-a) and who were not planning to quit (Dijkstra, De Vries, Roijackers \& Van Breukelen, in press-d). The computer program combined several potential parts of the message into one coherent intervention message and provided an atractive lay-out. Information in the tailored letters was adapted to the individual's stage of change, perceived outcomes, situational self-efficacy levels and smoking behavior. Most feedback was given on the item level. Decision rules were developed on the basis of theories of behavior change. Normative feedback, in all tailored conditions, was offered by comparing the individual's scale scores with the scale scores of smokers with the same plans to quit. The letter was personalized by including individual information, such as the name of the respondent, the number of cigarettes smoked per day, the number of years smoked and the amount of money to be saved in the case of quitting.

Content of the tailored interventions. The letters started with an introduction in which the rational of the content of the letter was explained. Depending on the individual item scores, the letters contained information on possible outcomes of smoking and quitting, such as personal health consequences, social consequences, such as appreciation of quitting by a non-smoking partner and the consequences of smoking for people in the environment. Restructuring information was offered with regard to the negative outcomes of quitting, such as weight gain, loss of functions of smoking, such as relaxation and expected withdrawal symptoms, . Furthermore, the letters contained information on skills to cope with social, emotional and habitual situations, depending on the individual confidence scores. The letters in the single tailoring conditions consisted of five to seven pages of information.

Multiple tailored interventions. The letters in the MT conditions contained roughly the same information as the letters in the ST conditions, but in the former the information was distributed over the three letters, each comprising four or five pages of information. Each of the three letters contained information on different outcomes of quitting and self-efficacy enhancing information, that is, skills. In the second and third letters in the MT conditions, subjects were offered feedback on the direction of cognitive changes, comparing their former stage of change and their former scores on the pros of quitting, the cons of quitting and self-efficacy expectations to the present scores on these variables. In the two MT conditions, the contents of the second and third letters were based on answers to a 10 -minute telephone questionnaire.

Tailored interventions plus self-help guide. In one of both the ST and the MT conditions, the letter(s) referred to the 46-page self-help manual which was sent simultaneously with the (first) letter. This self-help manual in color was developed to be used in a community smoking cessation project (Mudde, De Vries, Willemsen \& van Assema, 1994). In the introduction paragraph of letters, subjects were referred to a stage-matched chapter in the self-help manual. Furthermore, throughout the letter reference was made to additional information in the self-help manual. 


\section{Participant cllaracteristics}

Of the 752 respondents, $60.2 \%$ were female, $23.4 \%$ had low education, $40.4 \%$ medium and $36.2 \%$ high education and the mean age was 39.3 years. On the average they smoked 21.9 cigarettes a day, they had smoked for 21.5 years, the mean FTND score was 5.15 (scale ranging from $0-1.0$ ), and $77.3 \%$ were classified as immotive ( $22.7 \%$ precontemplator). Only $15.2 \%$ engaged in a quit attempt in the past twelve months. With regard to the cognitive measures, the mean scores on the pros and cons of quitting (scales ranging from $0-3$ ) were 1.19 and 1.09 respectively. The mean self-efficacy score (scale ranging -3 to + 3) was -0.47 and the mean intention score (scale ranging from 1-10) was 3.74 .

\section{Randomization and attrition}

In order to check the randomization, smokers in the five conditions were compared on the variables mentioned in the participant characteristics section. Chi-square tests for the discrete variables and F-tests for the continuous variables revealed no differences between the conditions, with one exception. Smokers in the NT condition were classified more often $(p<.05)$ as immotive.

Attrition from T1 to T2 was $19.6 \%(n=147$; MTplus $21.4 \%$; MTonly $12.2 \%$; STplus $17.8 \%$; STonly $25 \%$; NT 21.8\%). Logistic regression analysis with attrition as depenclent variable and the same variables as in the randomization check and Condition as predictors, revealed that drop-outs had smoked for fewer years $(p<.05)$, dropped-out more often $(p<.05)$ from the NT condition than from the MTonly condition and more often $(p<01)$ from the STonly condition than from the MTonly condition. Attrition in both MT conditions was $7.7 \%$ at the first telephone contact and, compared to the first telephone contact, $4.2 \%$ at the second telephone contact.

\section{Statistical analyses}

Logistic regression was used for the binary measures (stage transition, 24-hour quit attempt, seven days quit) and linear regression for the quantitative outcome measure (intention to quit). All regression analyses were corrected for Sex, Age, Education and Stage at pre-test. Because the intention to quit was not a meaningful measure anymore for participants who had quit smoking, participants who reported having refrained from smoking for the last 24 hours were excluded from the analyses on intention to quit.

First, in order to test whether tailored interventions have more effect than non-tailored interventions, the four tailored conditions were each compared to the non-tailored condition. The factor Condition was dummy-coded, using the NT condition as the reference condition with which each other condition was compared.

Second, in order to test whether multiple tailoring had more effect than single tailoring, the factor Times was computed (both MT conditions versus both ST 
conditions) and in order to test whether the combination of a tailored intervention with a self-help guide had any additional effect compared to a tailored intervention without this addition, the factor SHG was computed (both conditions with self-help guide versus both conditions without self-help guide). Times, $\mathrm{SHG}$, the interaction Times $\mathrm{x}$ SHG and the covariates were entered in the regression equation. The NT condition was excluded from these analyses. If the Times $x$ SHG interaction was significant $(p<.05)$, the analyses were stratified according to Times, otherwise the interaction was removed from the analysis. If the interaction was not significant, it was removed from equation and the main effects of Times and SHG were tested. In order to study to what extent the effectiveness of the conditions differed for subgroups of smokers, several interactions were tested. That is, the interaction of Condition with Stage, Sex, Age, Education, FTND score and number of cigarettes were entered in the equations. Because the latter two measures overlap, they were entered separately. In the case of a significant interaction $(j<.05)$, the analyses were stratified.

Participants in all five conditions were compared using linear regressions on the scores on the program evaluation questions, employing the same analysis strategy as for the outcome measures.

Table 8.1

Mean scopes on the progran evaluation items.

\begin{tabular}{lllll}
\multicolumn{4}{c}{ Conditions (N) } \\
\hline $\begin{array}{l}\text { MTplus } \\
\text { MTonly }\end{array}$ & STplus & STonly & NT \\
$(110)$ & $(137)$ & $(129)$ & $(114)$ & $(115)$ \\
\hline
\end{tabular}

To what extent (scale 1 to 10 ):

\begin{tabular}{|c|c|c|c|c|}
\hline did you read the letter? & $7.73^{b}$ & $8.54^{\mathrm{m}}$ & 7.75 & 7.99 \\
\hline do you remember the content? & 5.96 & $6.48^{\mathrm{a}}$ & 5.74 & 5.99 \\
\hline did you learn? & $5.18^{\mathrm{a}}$ & $5.54^{a}$ & 4.68 & 4.17 \\
\hline did you read the second letter? & 7.84 & 8.31 & & \\
\hline did you read the third letter? & $7.44^{b}$ & 8.19 & & \\
\hline did you read the self-help guide? & 7.13 & & 7.09 & \\
\hline $\begin{array}{l}\text { was the selfohelp guide beneficiall to } \\
\text { you? }\end{array}$ & 4.95 & & 4.78 & \\
\hline
\end{tabular}

Note. The evaluation in this condition differed significantly from the evaluation in the NTcondition."Both multiple talloring conditions differed signiflcanty from each other.

\section{Results}

\section{Program evaluation}

Table 8.1 shows the mean scores on the evaluation questions. First, comparing 
the four talored conditions to the NT condition, only smokers in the MTonly condition read significantly more of the (first) letter and reported remembering significantly more of it. Smokers in both MT conditions learned significantly more from the letters than smokers in the NT condition. Second, comparing both MT conditions, smokers in the MTonly condition read significantly more of the first and the third letter. Third, comparing the evaluation of the self-help guide in both conditions which offered the self-help guide, no significant difference was found.

\section{Tailoring versus non-tailoring}

Table 8.2 depicts the percentages (stage transition, quit attempt and seven days quit) and mean scores (intention) on the four outcome measures for each condition. None of the planned interaction tests was significant. Compared to the NT condition, both MT conditions led to higher percentages of transitions $(35.2 \%$ and $34.6 \%$ versus $18.6 \%$ ) and to a higher intention to quit (mean 15.4 and 14.5 versus 12.7 ). A contrast analysis revealed no significant difference between both MT conditions. The STplus condition and the MTonly condition led to higher percentages of 24 -hour quit attempts than the NT condition (13.4\% and $13.1 \%$ versus $6.1 \%$ ). A contrast analysis revealed no significant difference between both conditions. With regard to the seven days quit criterion, smokers in the MTonly condition reported, at borderline significance, having refrained more often from smoking for the past seven days $(7.4 \%$ versus $3.6 \%)$.

\section{Self-help guide versus no self-help guide}

In the two-way analyses of all tailored conditions, excluding the NT condition, there was no significant Times $x$ SHG interaction with regard to all four outcome measures. Therefore, the main effects of "Times and SHG were tested. Table 8.3 shows the percentages and means of the four outcome measures for the conditions with the additional self-help guide and for conditions without the self-help guide. There was a significant interaction between Condition and FTND score with regard to intention to quit and stage transition: in smokers who scored low on the measure of nicotine dependence $(\leq 5)$, the addition of the selfhelp guide did not lead to a significantly higher intention or to more stage transition. In smokers with high FTND score ( $>5)$, the addition of the SHO did lead to a significantly thigher intention to quit (Mean: 11.7 versus 14.1 ) and to significantly more stage transition $(20.6 \%$ versus $32.2 \%)$.

The data showed a borderline significant $(p<1)$ main effect with regard to the seven days quit criterion: the addition of a self-help guide led to fewer quilters $(4.4 \%$ versus $1.3 \%)$ 


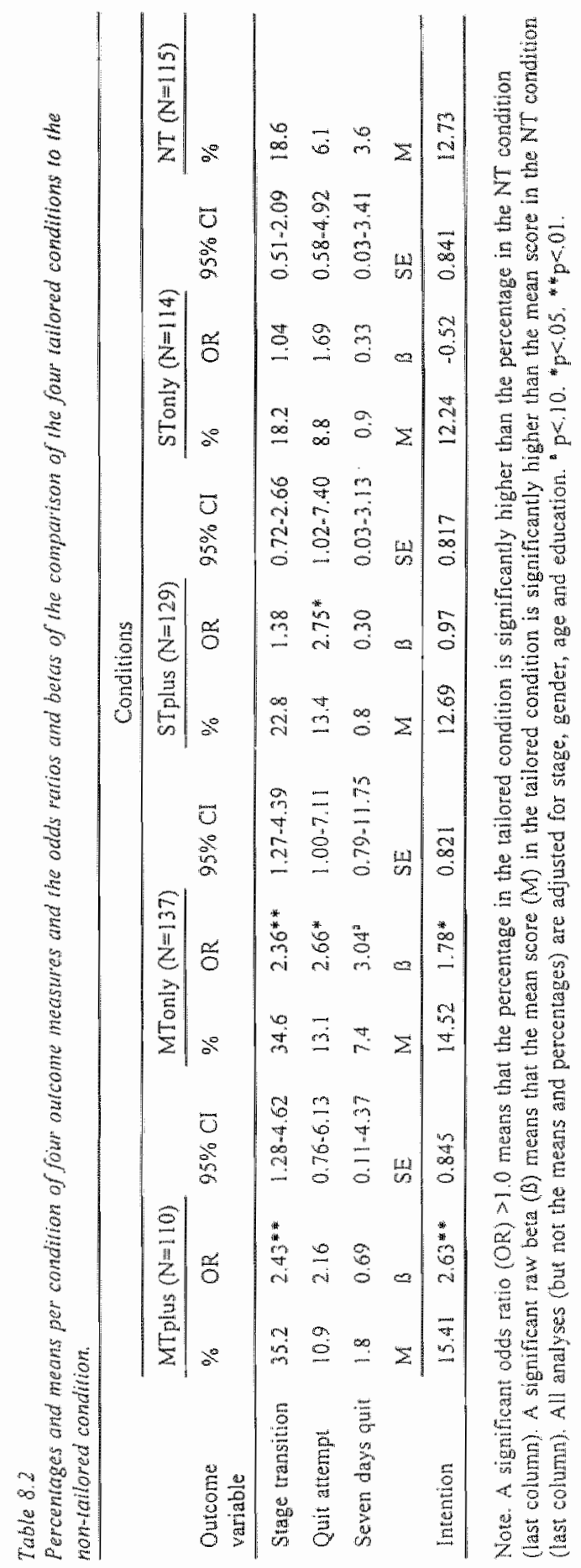




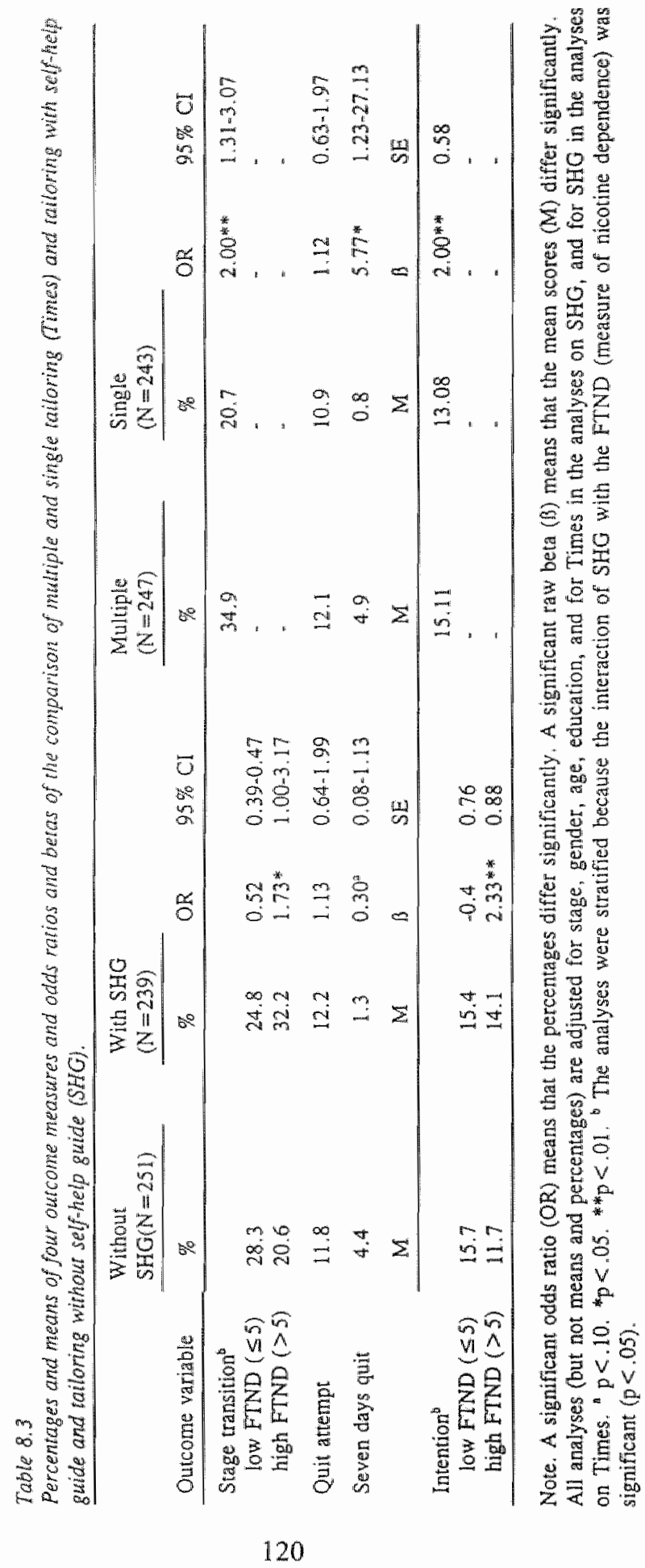




\section{Multiple tailoring versus single tailoring}

Table 8.3 depicts the percentages and mean scores for both MT conditions and both ST conditions. None of the planned interaction tests was significant. With regard to stage transition the effect of both MT conditions exceeded that of both $\mathrm{ST}$ conditions $(34.9 \%$ versus $20.7 \%)$. The same pattern was found with regard to the intention to quit (mean 15.1 versus 13.1 ) and the seven days quit criterion $(4.9 \%$ versus $0.8 \%)$. The MT conditions did not lead to significantly more 24 hour quit attempts than the ST conditions.

\section{Analyses including drop-outs}

Because attrition at $\mathrm{T} 2$ could be predicted by the number of years smoked as reported at $\mathrm{Tl}$ and by Condition, the present results might be influenced by selective drop-out. One way to address this problem is to use the last known measurement (TI) of each smoker who dropped out as a substitute for the $\mathrm{T} 2$ measurement and repeat all analyses. This 'intention to treat analysis" (Heyting, Tolboom \& Essers, 1992) revealed that none of the results changed qualitatively. Only minor changes in betas, odds ratios and p-values emerged.

\section{Discussion}

The first question was whether tailored information would lead to more changes than non-tailored information. The strictest comparison was between the single tailored intervention without the additional self-help guide and the non-tailored look-alike intervention. The data showed that the tailored intervention was not more effective than the non-tailored intervention. The results of the program evaluation may shed some light on the causes of this null finding. The progran evaluation data show that both single tailored interventions were read and remembered to the same extent as the non-tailored intervention. This contrasts with the findings of Skinner et al. (1994) and Campbell et al. (1994) and suggests that the tailoring of the information did not, as expected (Skinner et al., 1994; Dijkstra, 1996), lead to increased curiosity and a resulting enhanced attention to the message. First, the tailored intervention might not have been 'tailored enough'. However, the content was adapted to several individual cognitions and the intervention contained personal information, such as the name of the respondent, the number of cigarettes smoked a day, the number of years smoked and the amount of money saved in the case of quitting. Second, the fact that the single tailored intervention was not read and remembered to a larger extent than the non-tailored intervention might be caused by the specific sample of smokers with low motivation to quit: all smokers stated they were not planning to quit within the next six months and over $77 \%$ stated they were not planning to quit within the next five years. This group can be expected to be highly resistant to intention and behavior changing appeals. This resistance may have resulted in the finding that the tailored intervention was not read and 
remembered to a larger extent than the non-tailored intervention.

Both multiple talored interventions did lead to more stage transition and a higher intention to quit than the non-tailored intervention and the multiple tailored intervention without the additional self-help guide led, in addition, to more quitting behavior than non-tailored information. The power of multiple tailoring was supported in the analyses in which multiple tailoring was compared to single talloring. The data showed that on three out of four outcome measures, the effect of multiple tailoring exceeded that of single tailoring, while at the same time the letters in the multiple tauloring conditions contained roughly the same information as the letters in the single tailoring conditions. However, with the present study design the specific 'intervention elements' which are responsible for this effect of multiple tailoring can not be identified. For example, the effect of multiple tailoring might be caused by the fact that the information on the pros and cons of quitting and on the use of skills to quit was offered more than once. However, we do not know to what extent the tailoring of this information was essential. Three subsequent and related but non-tailored letters might have similar efficacy. Furthermore, we do not know to what extent the three assessments - one mailed questionuaire and two additional telephone interviews - contributed to the efficacy of multiple tailoring. Future research will have to address this important issue by comparing the effects of a multiple tailoring condition to, for example, the effects of a multiple but non-tailored condition and an assessment-only condition.

The addition of a standardized self-help guide to a tailored intervention led to a higher intention to quit but, in contradiction, to fewer seven days quitting (borderline significance) in comparison with a tailored intervention only. The results were more consistent when the analyses were stratified according to nicotine dependence: only in high nicotine dependent smokers did the addition lead to a higher intention to quit and to more stage transition. Strecher et al. (1994) found that in heavy smokers, taillored messages did not lead to more quitting than standardized or no information. They conclude that heavy smokers need nicotine replacement in addition to a psycho-social intervention. The present tindings, however, seem to support the notion that a more extensive psychosocial intervention might be needed to stimulate heavy smokers to quit. Both studies used different outcome measures and might be combined: in the present study the differential effect for low and highly dependent smokers was found on measures which, in a sample with low motivation to quit, are important in early phases of the process of smoking cessation. Strecher et al. used the seven-day quit criterion, which is important in later phases of the smoking cessation process. Hence, in motivating heavy smokers to quit, a more extensive psychosocial intervention might be needed, whereas in supporting actual quitting in heavy smokers, additional nicotine replacement might be needed.

In sum, in a critical comparison, the single tailored intervention without the additional self-help guide had no surplus value compared to a non-tailored lookalike intervention. The addition of a self-help guide to a tailored intervention was especially useful in highly dependent smokers. Multiple tailoring was clearly 
more effective than single tailoring.

The present study has its limitations. First, the follow-up was only four months. It cannot be ruled out that, for example, the addition of a self-help guide might be effective in the long rum. Second, we do not know how the present sample of mostly immotives compares to other samples with low motivation to quit as reported by Velicer, Hughes, Fava, Prochaska and DiClemente (1995) and Dijkstra et al. (in press-d). Consequently, the generalizability of the present results remains unclear. Third, smoking cessation outcomes and the program evaluation were measured at one time point. Hence, the program evaluation might be influenced by changes in intention and behavior.

Finally, the present study was designed to test whether certain tailored interventions are more effective than other interventions. In the development of tailored interventions this kind of research has to be supplemented with studies that try to unravel why some tailored interventions are more effective than others. Furthermore, future research should further address the question of for whom certain tailored interventions are more effective. The fact that in the present study a multiple tailored intervention was able to cause significant changes in intention and behavior in a sample with low motivation to quit, is a promising finding in the development of computer-generated tailored interventions. 


\title{
Chapter 9
}

\section{Targeting smokers with low readiness to change with tailored and nontailored self-help materials}

\begin{abstract}
Few smoking cessation self-help materials are available for smokers who are not planning to quit. However, computer-tailored interventions can specifically be designed for these smokers. In a large randomized field triall $(N=843)$, two different tailored smoking cessation self-help interventions (multiple tailoring and single tailoring) and one standardized smoking cessation self-help guide were compared to a no-information control group and to each other.

The standardized self-help guide had no effect. In smokers who were not planning to quit within the nexi five year (i.e. immotives), the multiple tailored intervention was more effective than the single tailored intervention. This pattern was supported by the cognitive changes caused by the interventions. In smokers who were planning to quit within the next five years but not within the next six months (i.e. precontemplators), none of the self-help materials had effect. The present results show that the self-help material currently available in the Netherlands, the standardized self-help guide, was not effective in smokers with low readiness to change. However, computer-generated tailored interventions seem a promising means of communicating information on smoking and smoking cessation to these smokers.
\end{abstract}

'This chapler is submited for pulatication as: Dijkstra, A., De Vries, H., \& Roijackers, J. (1997). Targering smokers wh low readiness ro change with ralowed and nontalored self-help materials. Manuscript subminted for publimation. Matastrich Universicy. 


\section{Introduction}

Self-help materials in smoking cessation are designed to support smokers in their further unaided quit attempts (Cummings, Emont, Jaèn \& Sciandra, 1988; Glynn, Boyd \& Gruman, 1990; Lichtenstein \& Glasgow, 1992). However, most of these self-help materials, however, are directed at smokers who are planning to quit. In the Netherlands, this means that there are no self-help or health education materials available for a large group of smokers: about $70 \%$ of smokers are not seriously planning to quit (Mudde, Dolders \& De Vries, 1994). Computertaillored self-help interventions in smoking cessation can be designed to take into account individual features, such as motivation to change, gender, foreknowledge and socio-demographic status (De Vries, Willemsen, Brug, Dijkstra \& Berben, 1995; Strecher et al., 1994; Velicer et al., 1993). In contrast to the standardized. (nontailored) self-help interventions, tailored self-help interventions can specifically be adapted to a state of low readiness to quit smoking. The present article reports on the effects of two computer-generated tailored self-help interventions and one nontailored self-help intervention in a sample of smokers with low readiness to change.

In testing self-help interventions, it is firstly important to know whether they are more effective than no intervention. That is, some studies show that smokers using self-help materials do not differ in their quitting behavior, or differ only in the short term, from smokers who quit without these materials (Curry, McBride, Grothaus \& Wagner, 1995; Owen, Ewins \& Lee, 1989). With regard to the effectiveness of tailored self-help interventions, a search of the literature reveals that a single tailored intervention can indeed lead to more changes in behavior than no information, after four to six months (Campbell et al., 1994; Kreuter \& Strecher, 1996; Strecher et al., 1994) but also after fourteen months (Dijkstra, De Vries \& Roijackers, in press-b).

Secondly, in testing self-help interventions, it is important to know which intervention is the most effective. That is, because self-help materials may differ in content, length or the frequency with which smokers are expected to use them, the effectiveness of these materials in a certain population may vary (Davis, Faust \& Ordentlich, 1984; Ershoff, Mullen \& Quinn, 1989; Dijkstra, De Vries \& Roijackers, 1996; Prochaska, DiClemente, Velicer \& Rossi, 1993). However, contrasting findings have been reported on the differing effectiveness of self-help materials. For example, Cummings et al. (1988) compared five selfhelp interventions with largely varying content and found no differences in effectiveness after six months (also see McFall et al., 1993). Conceming these differences it is particularly the difference in effectiveness between tailored and nontailored self-help interventions which is of interest. Studies addressing this issue show that tailored information can lead to more changes in behavior than nontailored information, but the effect may be limited to certain behaviors and subgroups (Brug, Glanz, Van Assema \& Kok, 1996; Brug, Steenhuis, Van Assema \& De Vries, 1996; Campbell et al., 1994; Dijkstra, De Vries \& Roijackers, 1996; Skinner, Strecher \& Hospers, 1994; Strecher et al., 1994). 
From a theoretical point of view, self-help interventions stimulate smokers to quit by changing the cognitive determinants of smoking cessation. Bandura's social cognitive theory (1986), among others (De Vries, Dijkstra \& Kuhlman, 1988; Prochaska, DiClemente \& Norcross, 1992), defines the perceived positive and negative outcomes of quitting and perceived self-efficacy as central cognitive determinants of motivation and behavior. Hence, self-help interventions are expected to increase the perception of positive outcomes of quitting, decrease the perception of the negative outcomes of quitting and to increase perceived self-efficacy. Furthermore, Bandura's social cognitive theory (1986), among others (Janis \& Mann, 1977; Prochaska et al., 1992), describes (in)attention as a means of blocking or processing potentially motivating information, for example, information on smoking and smoking cessation. In the transtheoretical model (Prochaska et al., 1992), the processes of change - operationalized as the self-reported frequency with which domain specific information is the focus of attention - are considered to be independent cognitive variables (Prochaska, 1984; Prochaska, Velicer, DiClemente \& Fava, 1988). Hence, self-help interventions are expected to increase the use of attentional processes. Several studies support the rellation between expected outcomes, selfefficacy and processes of change on the one hand, and motivation to quit and actual quitting on the other hand (De Vries \& Mudde, in press; Godin, Valois, Lepage \& Raymond, 1992; Mudde, Kok \& Strecher, 1995; Prochaska \& DiClemente, 1983; Prochaska, DiClemente, Velicer, Ginpil \& Norcross, 1985; Strecher, McEvoy DeVellis, Becker \& Rosenstock, 1986). However, no data are available on cognitive changes due to self-help interventions in smokers with low readiness to quit.

The first goal of the present study was to test the effectiveness of different self-help interventions in a sample of smokers with low readiness to change. Therefore, smokers were assigned to one of the following conditions which offered: I) three consecutive tailored letters; 2) one tailored letter; 3) one standardized self-help guide; 4) no information. The three self-help interventions were compared to the no-information control group and to each other.

The second goal of the present study was to analyze to what extent the selfhelp interventions were able to change the relevant cognitive determinants in a sample of smokers with low readiness to change. Again, the interventions were compared to the no-information control condition and to each other.

Because smokers with low readiness to change comprise a significant proportion of the smoking population, both in the Netherlands $(70 \%$; Mudde et al., 1994) and in the U.S. (40\%; Velicer et al., 1995), the research sample comprised only smokers who stated having no plans to quit within the next six months comprised the research sample. Both tailored interventions take into account the low motivation to quit, whereas the standardized self-help guide does not. Therefore, with regard to the effectiveness and the cognitive changes, it was expected that: 1) only the tailored interventions would lead to more changes than no information; 2) both tailored interventions would lead to more changes than the standardized self-help guide; 3) the tailored intervention 
consisting of three tailored letter would lead to more changes than the tailored intervention consisting of one tailored letter.

\section{Method}

\section{Participants}

Smokers were recruited by advertisements in local newspapers throughout the Netherlands, in which they were asked to volunteer for a research project on smoking and smoking cessation. Some specific measures were taken to recruit a sample of smokers with low intention to quit. First, in the advertisements the target group was defined as: "Smokers who are not planning to quit within the next six months, or may be planning to never quit". Second, to minimize a selective sampling, it was stated explicitly that smokers did not have to quit to join in the present study. Third, subjects completing all questionnaires were offered the opportunity to win 20 bonus prizes amounting to $\$ 100$.

After smokers had phoned the university in order to register $(n=1000)$, they were sent the pre-test questionnaire which could be returned in a pre-paid envelope. After two weeks, $915(91.5 \%)$ pre-test questionnaires had been returned. Seventy-two of these questionnaires were excluded, because the respondents only smoked a pipe or cigars, or because they did have plans to quit within the next six months. This resulted in 843 respondents at $\mathrm{Tl}(84.3 \%)$.

\section{Design}

Smokers were randomly assigned to one of four conditions (Figure 9.1) offering: 1) three (multiple) consecutive tailored letters (MT condition); 2) a single tailored letter (ST condition); 3) a standardized self-help guide (SHG condition); 4) no self-help materials (CO condition). Two weeks after the pre-test questionnaires $(\mathrm{T} I)$ were returned, the participants were sent the intervention material (in the MT condition, the first tailored letter) by mail. In the three experimental conditions the intervention was accompanied by a 1-page program evaluation questionnaire and a pre-paid return envelope. Six months after the intervention, 843 participants were sent the post-test questionnaire (T2) of which $750(89 \%)$ were returned. 


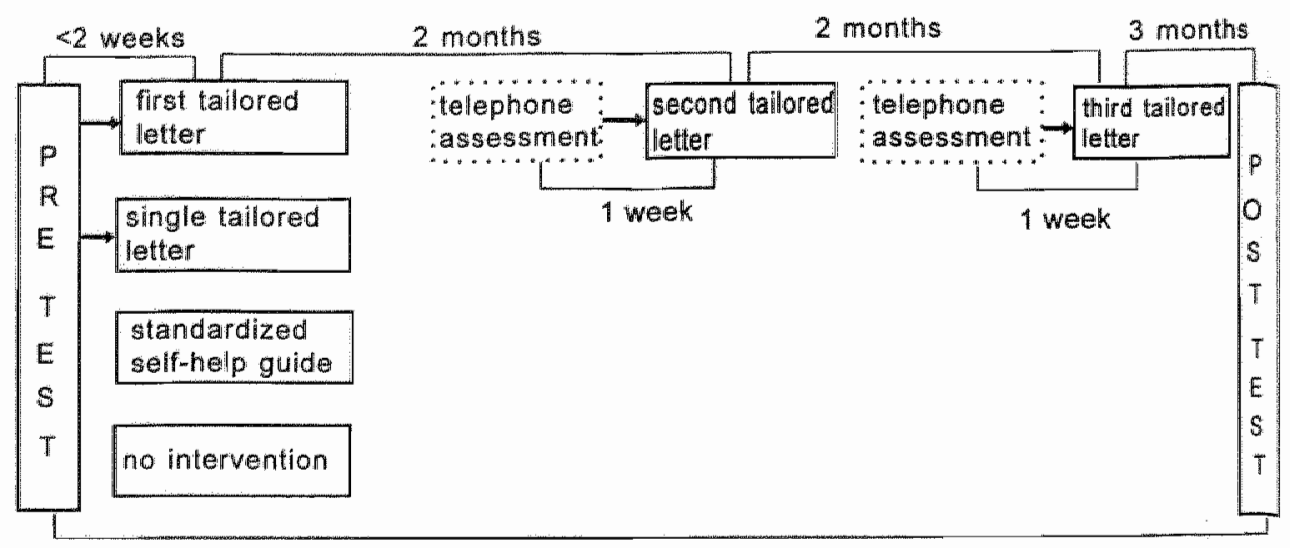

7 months

Figure 9.1 The design with three experimental conditions and one control condition.

\section{Questionnaire}

Because the present sample had a low motivation to quit at the start of this study with short follow-up, it was expected that only a few smokers would quit smoking. Hence, behavioral outcome measures were regarded as less appropriate. Stage-transition and intention to quit were considered to be the primary outcome measures (Velicer, Prochaska, Rossi \& Snow, 1992; Velicer, Rossi, Prochaska \& DiClemente, 1996).

Stages of change were assessed by confronting smokers with different plans with regard to smoking cessation (Dijkstra, Bakker \& De Vries, 1997). They were asked to score the one plan that was most similar to their own quitting plan: "Are you planning to quit within: 1) the next month; 2) the next six months; 3) the next twelve months; 4) the next five years; 5) somewhere in the future; 6) not planning to quit ever, but planning to cut down on cigarettes; 7) not planning to quit ever and not planning to cut down on cigarettes". The participants were, then, categorized in one of four stages of readiness to change: immotivation (plan 5,6 or 7), precontemplation (plan 3 or 4); contemplation (plan 2), preparation (plan 1). Smokers who had quit during the last 24 hours were considered to be in the action stage.

Stage transition was assessed by dichotomizing changes in stage: Forward transition was scored as 1 , versus no transition or backward transition which was scored as $0[16,29]$.

Intention to quit was measured with a composite of four 10-point scales: "Do you intend to quit smoking: 1) within the next month;2) within the next six 
months; 3) within the next five years; 4) ever?" The items coula be scored from "not at all" (1) to "very much" (10). The composite intention score was the average item score (possible range 1-10). Cronbach's reliability $(\alpha)$ was .78.

Quitting behavior was measured with a point prevalence measure: "Have you been smoking during the last seven days? (even one puff)" (yes/no).

In order to check whether the randomization procedure was successful with regard to some relevant determinants of quitting behavior, smokers in the four groups at T1 were compared on scale scores on positive and negative outcome expectations, perceived self-efficacy, attentional change processes, smoking behavior and demographics. The individual data were also used as the basis for the tailored interventions.

The items measuring the expected outcomes of quitting were validated in earlier studies (Dijkstra, De Vries \& Bakker, 1996; Dijkstra et al., in press-b), in which they were shown to have predictive and discriminative power with regard to intention to quit and with regard to smoking cessation. All outcome items referred to possible gains or losses anticipated by smokers as a consequence of quitting and were in the following format: "If I quit smoking, ......". The items could be scored firom "not sure" or "not expecting a certain outcome" (0) to a "strong expectation of the outcome" (3). The pros-of-quiting scale (positive outcome expectations) was composed of twelve items referring to the positive consequences of behavior change, such as the anticipated positive physical, social and self-evaluative consequences with regard to quitting. Cronbach's $\alpha$ was .87. The cons-of-quiting scale (negative outcome expectations) was composed of ten items referring to the negative consequences of behavior change $(\alpha=84)$, such as the loss of a means to relax and the loss of a means to cope with anger. The average item scores were used as the scale scores.

Twelve items assessed self-efficacy expectations with regard to the ability to refrain from smoking in social, emotional and habitual situations $(\alpha=94)$. The items and their format were validated in an earlier study in which they were predictive of smoking cessation (Dijkstra et al., in press-b). All self-efficacy expectations were measured on a 7-point scale and could be scored from "not sure at all I am able to refrain from smoking" (-3) to "very sure I am able to refrain from smoking" $(+3)$ and the average item scores were used as the scalle scores.

The attentional change processes were based on the Processes of Change construct (Prochaska, 1984; Prochaska et al., 1988). Prochaska and colleagues define and operationalize five experiential and five behavioral change processes: "... Which are overt and covert strategies and techniques used to modify problem behaviors" (p. 476; Prochaska, Redding, Harlow, Rossi \& Velicer, 1994). Taking into account the low motivation of the present sample, only the experiential processes were considered to be relevant. Three experiential processes were 
assessed": Consciousness Raising was assessed with questions, such as: "I recall articles dealing with the problem of quitting smoking" (4 items; $\alpha=82$ ). Environmental Reevaluation was assessed with questions, such as: "I consider the view that smoking can be harmful to the environment" ( 4 items; $\alpha=70$ ). Social Liberation was assessed with questions, such as: "I notice that public places have sections set aside for smoking" (4 items; $\alpha=.72$ ). Each scale could be scored in terms of frequency, from "never" $(0)$ to very often (3) and the average item scores were used as the scale scores.

Furthermore, smoking behavior was measured by asking smokers how many years they had been smoking, how many cigarettes they smoked, on average, and whether they had ever engaged in a 24-hour quit attempt in their lives and during the last 12 months. Finally, participants were asked whether their partner smoked (yes/no).

Demographics measured were sex, age and level of education. Level of education was categorized as low, medium, or high level of education. In the diverse schooling system in the Netherlands, the low level of education refers to vocational training, the medium level to advanced vocational training, and the high level to college/university training.

No biochemical verification of self-report quitting behavior was conducted, because it was expected that the announcement of a biochemical verification would increase nonresponse and drop-out, especially in the present sample with low motivation (Velicer, Prochaska, Rossi \& Snow, 1992). This would lead to a highly selective group of immotives and precontemplators. Moreover, the present study was characterized by low demand, that is, at recruitment smokers were told they did not have to quit smoking. For this reason the self-report was considered to be valid (Velicer et al., 1992).

\section{The tailored interventions}

General principles. The computerized system to generate the tailored letters was adapted from previous evaluations of minimal interventions in smokers who were planning (Dijkstra, De Vries \& Roijackers, in press-a) and who were not planning to quit (Dijkstra, De Vries \& Roijackers, 1996). The computer program combined several potential parts of the message to one coherent interwention message and provided an attractive lay-out. Information in the tailored letters was adapted to the individual's stage of change, perceived outcomes, situational self-efficacy levels and smoking behavior. Most feedback was given on the item.

\footnotetext{
According to Prochaska and collewgues the experiential processes are transtheoretical. In the present study. however, the change processes wert considered to refer to concrete and measurable allocations of attention. These wth ntional change processes ate considered to work as anformat ion filter which regulates, on the basis of a nect to process or aroid information. the frequency and time with which certain intormation is the conscious focus of atention. As such, these change processes are considered to be a cause of the processing of donntim-specitic information (1.e. unfornation on smoking and smoking cessation). Hence, wo of Prockaska's experiential processes, Self Reevaltation and Dramatic Relief, were not inclided in the present change process treasure: Self-Reewalustion and Dirnatic Relief seemed to assess an effect of the processing of domain-specific information, that is, fecting disappointed or beigg upset, rather than a cause of this processing.
} 
level. Normative feedback, in both tailored conditions, was offered by comparing the individual's scale scores with the scale scores of smokers with the sarne plans to quit. The letter was personalized by including individual information, such as the name of the respondent, the number of cigarettes smoked per day, the number of years smoked and the amount of money saved in the case of quitting. The tailored interventions were designed to increase the perception of positive outcomes of quitting, decrease the perception of the negative outcomes of quitting and to increase perceived self-efficacy.

Content of the tailored interventions. The letters started with an introduction in which the rational of the content of the letter was explained. Depending on the individual item scores, the letters contained information on possible outcomes of smoking and quitting such as personal health consequences, social consequences, such as appreciation of quitting by a non-smoking partner and the consequences of smoking for people in the environment. Restructuring information was offered with regard to the negative outcomes of quitting, such as weight gain, loss of functions of smoking, such as relaxation and expected withdrawal symptoms. Furthermore, the letters contained information on skills for coping with social, emotional and habitual situations, depending on the individual confidence scores. The letters in the single tailoring conditions consisted of five to seven pages of information.

Multiple tailored intervention. The letters in the MT condition contained roughly the same information as the letters in the ST condition, but in the former the information was distributed over the three letters, each comprising four or five pages of information. Each of the three letters contained information on different outcomes of quitting and self-efficacy enhancing information, that is, skills. In the second and third letters in the MT condition, subjects were offered feedback on the direction of cognitive changes, comparing their former stage of change and their former scores on the pros of quitting, the cons of quitting and self-efficacy expectations to the present scores on these variables. In the MT condition, the content of the second and third letters were based on answers in a 10-minute telephone questionnaire.

\section{Self-help guide}

The 46-page color self-help manual was developed for use in a community smoking cessation project (Mudde, De Vries, Willemsen \& van Assema, 1994). Its structure was derived from a self-help guide developed in the U.S. (Strecher, Rimer \& Monaco, 1989) and the language and content were adapted to the Dutch population. The manual consisted of the following chapters: Thinking about quitting; What keeps you from quitting?; Preparing a quit attempt; The first weeks without smoking; Relapsed; and The future without smoking. 


\section{Participant characteristics}

Of the 843 respondents, $62.8 \%$ were female, $29.1 \%$ had a low level of education, $44.8 \%$ a medium level and $26.1 \%$ a high level of education and the mean age was 41.7 years. On average, they smoked 21.5 cigaretes per day, they had smoked for 24.5 years, and $78.1 \%$ were classified as immotives $(21.9 \%$ precontemplators). $69.8 \%$ of the participants had engaged in a quit attempt in their lives and $35.2 \%$ had a smoking partner. The mean scores on the pros and cons of quitting and self-efficacy were $1.19,1.08$ and -0.71 , respectively. The mean scores on the three change processes, Consciousness Raising, Environmental Reevaluation and Social Liberation, were 1.01, 0.64 and 2.37, respectively. The mean intention score, on the scale from 1 to 10 , was 3.28 .

\section{Randomization and attrition}

To check the randomization, smokers in the four conditions were compared on the variables mentioned in the section on participant characteristics. Chi-square tests for the discrete variables and F-tests for the continuous variables revealed no significant differences between the conditions, with one exception. Tukey contrasts with regard to the average number of cigarettes a day showed that smokers in the SHG condition smoked significantly $(p<.05)$ less cigarettes per day $(M=20.0)$, than smokers in the $C O$ condition $(M=22.8)$. Hence, in all analyses the number of cigarettes per day was included as a covariate.

Altrition from $\mathrm{T} 1$ to $\mathrm{T} 2$ was $11 \%$ (n=93; MT condition $12.6 \%$; ST condition 12.2\%; SHG condition $6.9 \%$; $\mathrm{CO}$ condition $12.5 \%$ ). Log ${ }^{\prime \prime s t i c}$ regression analysis with attrition as the dependent variable and with the predictors being the same variables as in the randomization check and Condition, revealed that drop-outs had a significantly $(p<.05)$ lower self-efficacy at $\mathrm{T} 1$.

At the first telephone contact, $4.2 \%$ had dropped out of the MT condition, while at the second telephone contact $9.3 \%$ had dropped out since the first telephone contact.

\section{Statistical analyses}

Logistic regression was used for the binary outcome measures (stage transition, seven days quit) and linear regression for the quantitative outcome measure (intention to quit). In the case of a significant main effect of one of the variables, Sex, Age, Education, Stage, Partner (having or not having a smoking partner) or Lifetime quit (having or not having engaged in a quit attempt in their life), the variable was included in the analysis as a covariate. In all analyses, the number of cigarettes a day was entered as a covariate. The factor Condition was dummy-coded. For all tests, $\alpha=.05$ was used. Because the intention to quit was not a meaningful measure anymore for participants who had quit smoking, participants who reported having refrained from smoking during the last seven days were excluded from all analyses on intention to quit. 
To test whether the conditions led to differential outcomes, first, an overall F-test was conducted for each outcome measure. Second, contrasts between the four conditions were computed: 1) the three self-help interventions were each compared to the no-information control condition (the control condition being the reference group); 2) both tailored interventions were each compared to the standardized self-help guide (the latter condition being the reference group); 3) the multiple tailored intervention was compared to the single tailored intervention (the single tailored condition being the reference group). To study to what extent the effectiveness of the conditions differed for subgroups of smokers, several interactions were tested. That is, the two-way interaction of Condition with Sex, Age, Education, Stage, Partner, Lifetime quit and the number of cigarettes a day were entered in the equations. Non-significant interactions were removed stepwise from the analyses. In the case of significant interactions remaining $(p<.10)$, the analyses were stratified.

To test to what extent the self-help interventions changed the relevant cognitive factors differentially, the conditions were compared on changes in the pros and cons of quitting, perceived self-efficacy and the three attentional change processes. First, an overall F-test was conducted. Second, the experimental conditions were compared to the no-information control condition and to each other. The Tl scores were entered as covariates. To rule out the cognitive changes being caused by changes in behavior, smokers who at $\mathrm{T} 2$ stated having refrained from smoking for seven days were removed from these analyses.

\section{Results}

\section{The effectiveness of self-help interventions}

Table 9.1 shows the percentages and means and the overall F-tests of the comparison of the four conditions on the three outcome measures. Only the overall tests for the outcome measures which were considered to be appropriate for the present sample with low motivation to quit, stage transition and intention to quit, were significant. With regard to intention to quit and seven-days quit, none of the interactions tested was significant. With regard to stage transition, a significant Stage $X$ Conditions interaction emerged $(\mathrm{p}<.001)$ meaning that, for immotive and precontemplating smokers, the conditions differed in effectiveness. Hence, the analyses with regard to stage transition were stratified according to stage. Table 9.2 shows the results of the contrasts between the conditions. With regard to the behavioral outcome measure, none of the contrasts was significant.

First, all three experimental conditions were compared to the no-information condition. With regard to stage transition in immotives, both tailored interventions led to significantly more stage transition than no information (42. $1 \%$ and $26.1 \%$ versus $14.3 \%$ ), whereas the self-help guide did not. With regard to stage transition in precontemplators, none of the experimental 
Table 9.1

Percentages and means per condition and overall significance with regard to three ontcome measures.

\begin{tabular}{|c|c|c|c|c|c|}
\hline \multicolumn{6}{|c|}{ Conditions } \\
\hline Outcome variable & $\begin{array}{l}\mathrm{MT} \\
(\mathrm{N}=187)\end{array}$ & $\begin{array}{l}\mathrm{ST} \\
(\mathrm{N}=180)\end{array}$ & $\begin{array}{l}\text { SHG } \\
(N=201)\end{array}$ & $\begin{array}{l}\mathrm{CO} \\
(\mathrm{N}=182)\end{array}$ & $\begin{array}{l}\text { Owerall } \\
\text { F-test }\end{array}$ \\
\hline Stage transition(\%) & & & & & \\
\hline immotives & 42.1 & 26.1 & 20.5 & 14.3 & $<.001$ \\
\hline precontemplators & 25.6 & 35 & 19 & 40 & ns \\
\hline Seven days quit( $\%$ ) & 3.2 & 4.4 & 3.5 & 5.5 & ns \\
\hline Intention(Mean) & 4.35 & 3.98 & 3.8 & 3.55 & $<.01$ \\
\hline
\end{tabular}

Note. All analyses (but not the means (M) and percentages) are adjusted for number of cigarettes smoked a day. $\mathrm{MT}=$ multiple tailored intervention; $\mathrm{ST}=$ single tailored intervention; $\mathrm{SHG}=$ self-help guide; $\mathrm{CO}=$ no-information control group.

conditions was more effective than no information. The self-help guide even led to significantly less stage transition than no information in precontemplators $(19 \%$ versus $40 \%)$. With regard to intention to quit, the multiple tailoring condition led to a significantly higher intention and the single tailoring condition to a borderline significantly $(p<.10)$ higher intention $(4.35$ and 3.98 versus 3.55$)$. The difference between self-help guide and no information was non-significant.

Second, both tailored interventions were compared to the self-help guide. With regard to stage transition in immotives, only the multiple tailoring condition was significantly more effective than the self-help guide $(42.1 \%$ versus $20.5 \%)$. With regard to stage transition in precontemplators, no significant differences were found between both tailored interventions and the self-help guide. With regard to intention to quit, only multiple tailoring led to a significantly higher intention than the self-help guide ( 4.35 versus 3.8 ).

Finally, both tailored interventions were compared to each other. With regard to stage transition, only in immotives was multiple tailoring significantly more effective than single tailoring ( $42.1 \%$ versus $26.1 \%$ ). With regard to intention to quit, multiple tailoring was borderline significantly more effective than single tailoring ( 4.35 versus 3.98 ).

\section{Analyses including drop-outs}

Because attrition at $T 2$ could be predicted by perceived self-efficacy at $T 1$, the present results might be influenced by selective drop-out. One way to address this problem is to use the last known measurement (TI) of each smoker who dropped out as a substitute for the $\mathrm{T} 2$ measurement and repeat all analyses. This "intention to treat analysis" (Heyting, Tolboom \& Essers, 1992) revealed that none of the results changed qualitatively. Only minor changes in betas, odds ratios and p-vallues emerged. 


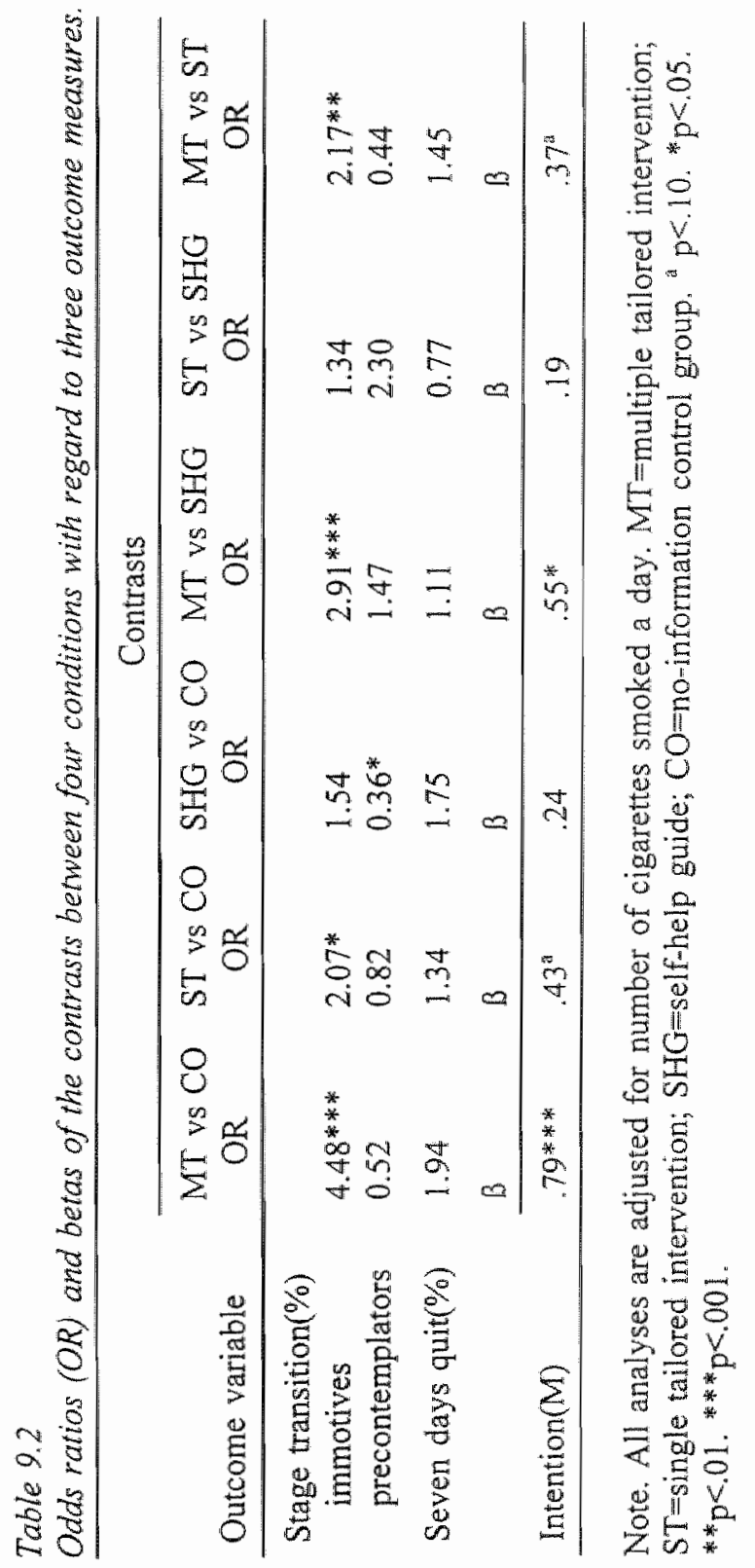




\section{Cognitive changes resulting from the self-help interventions}

To rule out that cognitive changes were caused by behavior change instead of by the interventions, smokers who at $\mathrm{T} 2$ stated having refrained from smoking for seven days were removed from these analyses. To test whether any cognitive changes had occurred at all, a paired T-test was conducted for each cognitive measure. These tests showed a significant increase between $\mathrm{T} 1$ and $\mathrm{T} 2$ in the pros of quitting, selfefficacy, Consciousness Raising and Environmental Reevaluation and a significant decrease in the cons of quitting and Social Liberation. Table 9.3 shows the means at $T 2$ and the overall $F$-tests of the comparison of the four conditions on the six cognitive measures. The conditions did, overall, lead to significantly different changes in the perception of the pros of quitting, self-efficacy and the use of the change processes of Consciousness Raising and Environmental Reevaluation. None of the interaction tests (two-way interactions of Condition with Sex, Age, Education, Stage, Partner, Lifetime quit and the number of cigarettes a day) was significant. Hence, the analyses were not stratified. Table 9.4 shows the results of the contrasts between the conditions. With regard to the cons of quitting and Social Liberation, none of the contrasts was significant at the .05 level.

Table 9.3

Means at $T 2$ per condition and overall significance with regard to six cognitive measures.

\begin{tabular}{llllll}
\hline \multicolumn{7}{c}{ Conditions } \\
\hline Cognitive variable & $\begin{array}{c}\mathrm{MT} \\
(\mathrm{N}=181)\end{array}$ & $\begin{array}{c}\mathrm{ST} \\
(\mathrm{N}=172)\end{array}$ & $\begin{array}{c}\mathrm{SHG} \\
(\mathrm{N}=194)\end{array}$ & $\begin{array}{c}\mathrm{CO} \\
(\mathrm{N}=171)\end{array}$ & $\begin{array}{l}\text { Overall } \\
\text { F-test }\end{array}$ \\
\hline Pros of quitting & 1.44 & 1.36 & 1.33 & 1.31 & $\mathrm{p}<.05$ \\
Cons of quitting & .93 & .95 & 1.02 & .99 & $\mathrm{~ns}$ \\
Self-efficacy & .16 & -.41 & -.42 & -.45 & $\mathrm{p}<.001$ \\
Consciousness Raising & 1.48 & 1.33 & 1.18 & 1.09 & $\mathrm{p}<.001$ \\
Environmental Reev. & .80 & .71 & .69 & .63 & $\mathrm{p}<.05$ \\
Social Liberation & 2.31 & 2.30 & 2.32 & 2.30 & $\mathrm{~ns}$ \\
\hline
\end{tabular}

Note. All analyses and means (M) are adjusted for rumber of cigarettes smoked a day and the pre-test score on the cognitive variable. $\mathrm{MT}=$ multiple tailored intervention; $\mathrm{ST}=$ singlle vailored intervention; $\mathrm{SHG}=$ self-help guide; $\mathrm{CO}=$ no-information control group. Smokers who reported having quil for the last seven days were excluded from the analyses. 


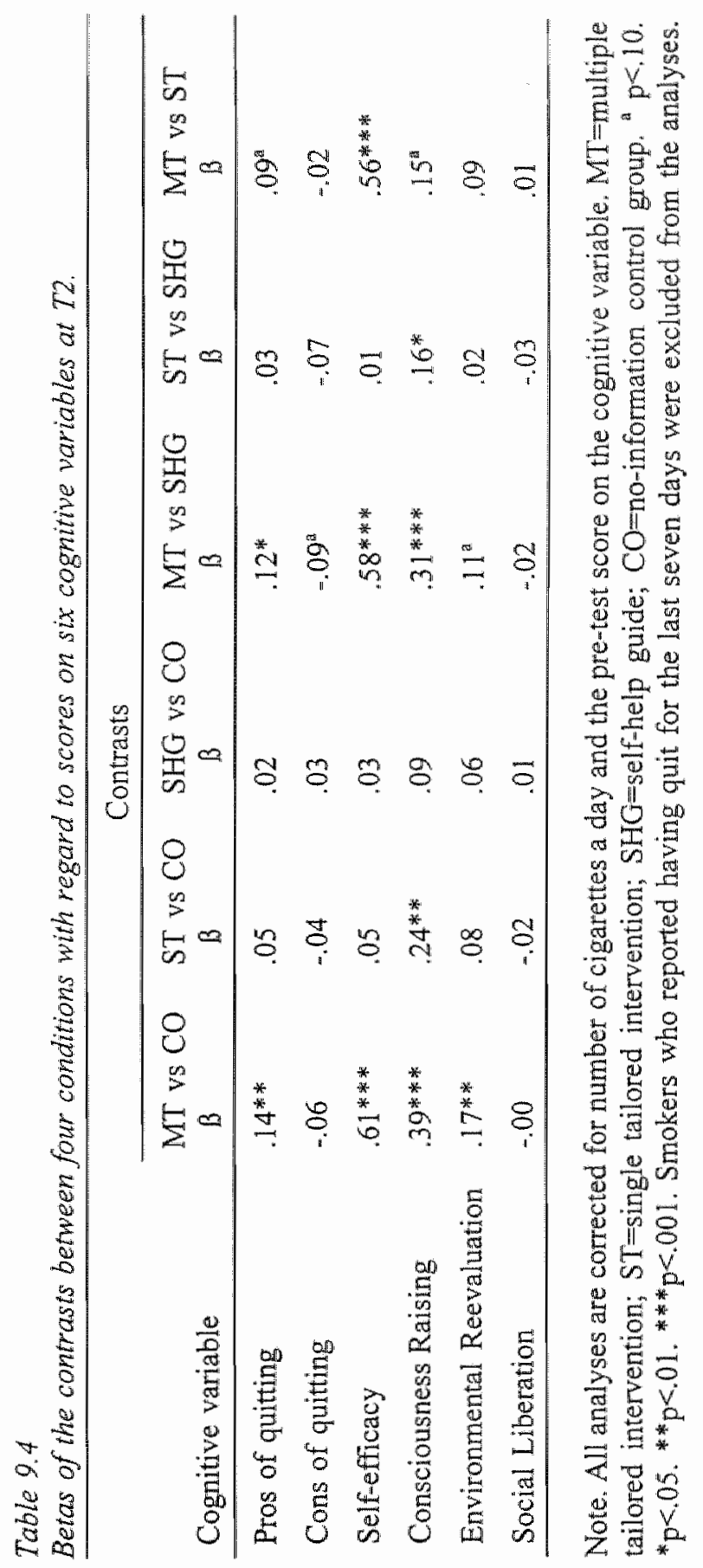


Pros of quitting. Multiple tailoring led to a significantly higher score on the pros than the control condition, whereas the single tailoring and the self-help guide did not. Multiple tailoring led to a significantly higher score on the pros than the self-help guide, whereas the single tailoring did not. Furthermore, multiple tailoring led borderline significantly higher score on the pros than single tailoring.

Self-efficacy. Multiple tailoring led to a significantly higher self-efficacy score than the control condition, whereas the single tailoring and the self-help guide did not. In addition, multiple tailoring led to a significantly higher self-efficacy score than the self-help guide, whereas the single tailoring did not. Furthermore, multiple tailoring led to a significantly higher self-efficacy score than single tailoring.

Consciousness Raising. Both tailored interventions led to a significantly higher score on Consciousness Raising than the control condition, whereas the self-help guide did not. Both tailored interventions also led to a significantly higher score on Consciousness Railsing than the self-help guide. Furthermore, multiple tailoring led to a borderline significantly higher score on Consciousness Raising than single tailoring.

Environmental Reevaluation. Multiple tailoring led to a significantly higher score on Environmental Reevaluation than the control condition, whereas the single tailoring and the self-help guide did not. In addition, multiple tailoring led to a borderline significantly higher score on Environmental Reevaluation than the self-help guide, whereas the single tailoring did not. Furthermore, both tailored interventions did not lead to significant differences with regard to Environmental Reevaluation.

\section{Discussion}

Given the present sample had low motivation to quit, stage transition and intention to quit were considered to be the most appropriate outcome measures. The fact that the behavioral measure showed no significant effects at all seemed to support this notion. The data from the intention to quit measure were similar to that of stage transition in immotives. Since almost $80 \%$ of the sample was classified as immotive, it is plausible that the data on intention to quit reflect the intervention effects in immotives. Therefore, with regard to the effectiveness of the interventions, the stratified results on stage transition will be discussed. The main findings with regard to the effectiveness can, thus, be summarized as follows.

In immotives, both tailored interventions but not the standardized self-help guide, had more effect than no information. Thus, although the self-help guide clearly offered more information on smoking and smoking cessation than the tailored interventions, it did not lead to more stage transition. Hence, it might be concluded that in immotives in the present sample the amount of information was not the crucial factor in interventions to encourage smokers to quit. This interpretation is supported by earlier findings of no differences in effectiveness 
between self-help materials of about 50 pages and a self-help booklet of 15 pages (Cummings et al.., 1.988).

Comparing the three self-help interventions in immotives, multiple tailoring had more effect than the self-help guide and the single tailored intervention. The latter finding is in line with findings from an earlier study, in which multiple tailored interventions were clearly more effective than single tailored interventions (Dijkstra, De Vries, Roijackers \& Van Breukelen, in press-e). The greater effect of the multiple tailored intervention might be attributed to one of the differences between this intervention on the one hand and the other self-help interventions on the other. First, the feedback received on the changes in perceived self-efficacy, the anticipated pros and cons of quitting and quitting plans could have led to the greater effect. Second, the greater effect may have been caused by the fact that information on the pros and cons of quitting and on the use of skills to quit was processed more than once, thereby increasing the frequency with which relevant information was the focus of attention. Third, the two additional telephone assessments could have caused the effect: being asked to elaborate again on personal motives for smoking or quitting may have resulted in increased processing of information relevant to smoking cessation. Furthermore, the two additional contacts with the telephonist might have led to a higher commitment to the perceived goals of the study. However, Brug and colleagues found that multiple tailoring led to more changes in dietary behavior than single tailoring, despite the fact that in both conditions three assessments were conducted (Brug, Glanz et al., 1996).

Although the direct comparison in immotives between the single tailored intervention and the self-help guide showed no difference, the single tailored intervention had more effect than no information, whereas the self-help guide did not. Furthermore, whereas the standardized self-thelp guide even led to significantly fewer stage transitions than no information in precontemplators ( $19 \%$ versus $40 \%$ ), the single tailored intervention did not show this effect $(35 \%$ versus $40 \%$ ). Thus, on the basis of these findings, the single tailored intervention would be the intervention of choice to encourage smokers with low readiness to change.

In precontemplators, the effects of the interventions were conspicuous by their absence. This is a puzzling finding since immotives were considered to be more resistant to appeals to change behavior than precontemplators. The present findings suggested the reverse, however. In precontemplators, the 46-page standardized self-help guide even led to fewer stage transitions than no information. Regardless of the fact that this difference was significant, offering the self-help guide reduced the percentage of stage transitions from $40 \%$ to $19 \%$. In an earlier study (Dijkstra et al., 1996), about $17 \%$ of immotives and $17 \%$ of precontemplators made a forward stage transition in a no-information control group, three months after an intervention. In the present study these percentages were $14.3 \%$ and $40 \%$, respectively. In other words, among the precontemplators in the present study the percentage in the control group seemed exceptionally high. To check whether this was due to pre-test differences between the four 
conditions, precontemplators in the four conditions were compared on all the variables which were used in the randomization check. None of the variables discriminated between the groups. Similarly, Curry et al. (1995) found a high percentage of quitters in their no-information control group and they attributed this to the continued social and normative pressure against smoking. In the present study, this would mean that precontemplators, but not immotives, were sensitive to social and normative pressure. As a result, precontemplators may have felt encouraged to quit smoking at the moment they joined the study, whether they received self-help materials or not. Post-hoc analyses showed that, overall, precontemplators processed more information relevant to smoking cessation, that is, they scored significantly higher on Consciousness Raising and Environmental Reevaluation than immotives. In other words, precontemplators may have been more sensitive to social and normative pressure because they process more of this information, thereby decreasing relatively the effect of the self-help interventions. Future studies will have to further explore the effectiveness of interventions in smokers in different stages of readiness to change.

The main findings with regard to the cognitive changes can be summarized as follows. The 46-page self-help guide did not lead to anymore cognitive changes than no information. In contrast, the single tailored intervention consisting of 5 to 7 pages, did lead to more Consciousness Raising than no information and the self-help guide. Thus, the single tailored intervention seemed to have characteristics that increased the frequency with which domainrelevant information (information on smoking and smoking cessation) is the focus of attention, even after seven months. The multiple tailored intervention led to larger changes in the pros of quitting, self-efficacy, Consciousness Raising and Environmental Reevaluation than no information and the self-help guide. Again, the self-help intervention consisting of three subsequent and related tailored mailings was the most powerful cognitive changer.

The perceived cons of quitting and the change process of Social Liberation decreased from $\mathrm{T} 1$ to $\mathrm{T} 2$ but they were not differentially influenced by the conditions. The cons of quitting refer to the loss of the functions of smoking, which may be overleaned and hard to change (Beck, Wright, Newman \& Liese, 1993), especially by (minimal) self-help intervention. Social Liberation was assessed as the frequency with which smokers, for example, 'notice "No Smoking" signs in public places". The present null-finding stresses the need for further validation of Social Liberation as a change process. Moreover, the literature on this change process reveals that in some cases it is positively related to the process of behavior change (Fava, Velicer \& Prochaska, 1995; Prochaska et al., 1992) and in others it is not (DiClemente et al., 1991; Kristeller, Rossi, Ockene, Goldberg \& Prochaska, 1992; Prochaska, Velicer, Guadagnoli, Rossi \& DiClemente, 1991).

The present study had its limitations. First, the follow-up period was only seven months. It may be so that, for example, the self-help guide would have effects in the longer term, when smokers become more motivated to process 
information on smoking and smoking cessation. Second, the present study was designed to answer whether certain self-help interventions were nore effective than others. Future studies will have to address more systematically why some self-help interventions are more effective than others.

In conclusion, the expectations with regard to the changes caused by the interventions were to a large extent met although this was only the case for smokers who were not planning to quit within the next five year (i.e. immotives). In smokers who were planning to quit within next five years but not within the next six months (i.e. precontemplators), the self help materials had no effect. With regard to the cognitive changes, the multiple tailored intervention was clearly the most effective.

The present results show that the self-help materials currently available in the Netherlands are not effective in smokers who are not planning to quit in the next six months but that tailored interventions can be effective in immotives. Although tailoring health education information is still in its infancy, the present study shows that computer-generated tailored interventions are a promising means of communicating information on smoking and smoking cessation to smokers with low readiness to change. 


\section{Chapter 10}

\section{General discussion and integration}

\section{Introduction}

The purpose of this final chapter is to relate and integrate the findings of the different studies. To this end, the findings from the eight studies presented in this thesis will be summarized and discussed.

In the first part of this chapter, the results from three studies in this thesis on the cognitive determinants of readiness to change and smoking cessation will be discussed. Specifically to be addressed will be the extent to which different sorts of outcome and self-efficacy expectations were related to the stages of readiness to change. Furthermore, the model of stages of readiness to change which distinguishes between four instead of three early stages will be discussed. In the second part of this chapter, the results from the four studies on the effectiveness of the tailored interventions will be discussed. Kazdin's (1986) evaluation strategies are used to structure the findings. The results in this thesis must be interpreted against the background of the soundness of the methodology used. Therefore, in the third part of this chapter some methodological issues will be discussed. In the fourth part, some applications of the theoretical findings and of the tailored interventions will be addressed. In the last part of this chapter recommendations with regard to future studies on the cognitive determinants, the stages of change and the effectiveness of tailored interwentions, will be given. 


\section{Determinants of readiness to change and smoking cessation}

\section{Chapter 2: The patterns of cognitive determinants through the stages.}

In Chapter 2, smokers in precontemplation, contemplation, preparation, action and maintenance were compared on positive and negative outcome expectations (pros and cons of quitting) and self-efficacy expectations.

The main goal was to study the pattern of the pros and cons of quitting and self-efficacy through the stages. With regard to the pattern of the pros of quitting and self-efficacy we expected to find the $\varnothing$-pattern (De Vries \& Backbier, 1994): the pros of quitting would discriminate better between the early stages (precontemplation, contemplation and preparation), whereas self-efficacy expectations would discriminate better between the later stages (action and maintenance). The $\emptyset$-pattern was indeed replicated. This differential pattern of the pros of quitting and self-efficacy through the stages could have consequences for stage-matched interventions: precontemplators might need information on the positive outcomes of quitting to move forward through the stages, contemplators might need this outcome information combined with self-efficacy enhancing information and preparers might need only self-efficacy enhancing information. Similarly, according to Janis \& Mann (1977), in a sound decision making process, people first process information on the consequences of changing or not changing the behavior and after that they process information on how to change the behavior. However, social cognitive theory (SCT) posits at least two causes of low motivation to change: the anticipation of few positive outcomes of quitting could lead to low motivation, whereas low self-efficacy would lead to decreased availability of the positive outcomes of quitting and, hence, to low motivation. In other words, in SCT, some precontemplators might need information on the positive outcomes of quitting, some might need only selfefficacy enhancing information and some might need both sorts of information. It could be expected that such a heterogeneous population as a whole would benefit most from a combination of both sorts of information. In the study in Chapter 6 the contrasting hypotheses - the $\varnothing$-pattern versus the expectations based on SCT - were tested.

With regard to the pattern of the pros and cons through the stages we expected to find the "strong and weak principle of behavior change" (Prochaska, 1994): the maximum difference within the first four stages (precontemplation, contemplation, preparation and action) on the pros of quitting was expected to be about 1. standard deviation and the maximum difference within these stages on the cons of quitting was expected to be about 0.5 standard deviation. One standard deviation change is considered to represent a large or "strong" effect, while 0.5 standard deviation change is considered to represent a medium-sized or "weak" effect (Prochaska, 1994). The present study did indeed replicate the "strong and weak principle of behavior change". The regularity of the patterns of the pros and cons of quitting through the stages is intriguing - they are replicated in several behaviors (Prochaska et al., 1994) and crossmculturally in the present 
study. However, Prochaska's interpretation of the differential effects of the pros and cons of seems to go beyond the data. First, although all data on the strong and weak principle are cross-sectional, Prochaska (1994) makes longitudinal and causal claims. Second, the transtheoretical model (TTM) explicitly recognizes the maintenance stage. However, in the computations of the strong and weak principle this stage was not included, although the cons seem to further decrease from action to maintenance (see Prochaska et al., 1994). When the maintenance stage was taken into account in the study in Chapter 2 , the maximum difference with regard to the cons of quitting did indeed increase from 0.5 standard deviation to 0.8 standard deviation. In other words, the cons of quitting became less "weak". Third, Marlatt and Gordon (1985) consider positive expectations with regard to the function of the unhealthy behavior to be a central cause of relapse. Positive expectations with regard to the functions of the unhealthy behavior can be considered to refer to the same phenomenon as the negative expectations of changing the unheal thy behavior (Prochaska et al., 1994). Hence, the cons of quitting refer to this central cause of relapse and by speaking of 'the weak principle of behavior change", the importance of expectations with regard to the loss of function may be strongly underestimated. Therefore, it may be more appropriate to speak of the early and late principle of behavior change: an increase in the pros of quitting would be needed in the early stages to increase motivation or readiness to change behavior, whereas a decrease in the cons of quitting would be needed in the later stages to prevent relapse.

In addition, this finding with regard to the differential pattern of the pros and cons of quitting through the stages, stresses the utility of the distinction between positive and negative outcome expectations. Positive and negative outcome expectations of behavior change (advantages/disadvantages; pros and cons of changing) are often regarded as the cognitive parameters of attitude (De Vries \& Backbier, 1994; Eagly \& Chaiken, 1993; Stroebe \& Stroebe, 1995). This conjunction of both concepts in the attitude concept seems to conceal the difference between the anticipated positive and negative consequences of behavior change.

Based on these findings with regard to the pros and cons of quitting and selfefficacy through the stages, the following can be concluded: First, the findings indicate that these constructs refer to the same psychological phenomena as those in the TTM. Second, because almost all studies on the Stages of Change are based on U.S.A. populations, this study can be regarded as a cross-cultural replication of the Stages of Change.

\section{Chapter 3: The stage model with smokers in four stages.}

Precontemplators in Chapter 2 indicated that they were not planning to quit within the next six months. The question which then arises is whether they have any plans at all with regard to changing their smoking behavior. An earlier study showed that within the group of precontemplators two subgroups can be distinguished on the basis of their quitting plans: immotives, who were not 
planning to quit within at least the next five years and (newly defined) precontemplators, who were planning to quit within that period of time (Dikstra, Bakker \& De Vries, 1997). Thus, the a forementioned study recognized smokers in four stages of increasing readiness to quit: immotives, precontemplators, contemplators and preparers.

The main goal of Chapter 3 was to replicate and expand the earlier study which distinguished between the four groups (Dijkstra et al, 1997). The first way of investigating the four stages was to compare them on the scores on the cognitive determinants. To obtain a more differentiated view of the cognitive differences, the four stages were compared on the scores on the factors within the pros and cons of quitting and selfeefficacy.

A principal component analysis with regard to the pros of quitting revealed the following components: long-term health consequences, short-term health consequences, social consequences and self-evaluative consequences. In the study in Chapter 4 these factors differed largely in the extent to which they were predictive of intention to quit. That is, the perceived self-evaluative consequences had the strongest positive relation with intention to quit, whereas the perceived long-term health consequences had the weakest positive relation with intention to quit. Within the items assessing self-efficacy, two factors were found: self-efficacy with regard to coping with the urge to smoke in social situations and self-efficacy with regard to coping with the urge to smoke in emotional situations. The study in Chapter 7 further verified the distinction between both sorts of self-efficacy: self-efficacy in emotional situations was predictive of continuous abstinence, whereas self-efficacy in social situations was predictive of 24-hour quit attempts. In the search for determinants of readiness to change, it was concluded that, besides the distinction between the positive and negative outcome expectations, there may be a useful distinction between physical, social and self-evaluative outcome expectations and between selfefficacy expectations with regard to different tasks.

The second way to investigate the four stages was to test to what extent stage membership at T1 was predictive of smoking cessation after three and after fourteen months. The results with regard to the cognitive differences between the stages and the predictive validity can be summarized as follows: immotives and precontemplators differed on all cognitive factors determined with analyses of variance, and the distinction between both stages was highly predictive of later quitting behavior determined with regression analyses. Precontemplators and contemplators differed on several cognitive factors, but the distinction between both stages was moderately predictive of quitting behavior. Contemplators and preparers differed on only one cognitive factor, but the distinction between both stages was highly predictive of quitting behavior. The data from the effectiveness studies partly supported the distinction between the four stages. The findings in Chapter 9 did, however, support the distinction between immotives and precontemplators: in immotives the self-help interventions led to more stage transition than no information, whereas in precontemplators this was not the case. The findings from the effectiveness study in Chapter 6 only 
qualitatively supported the distinction between precontemplators and contemplators: the condition offering a combination of outcome information and self-efficacy enhancing information led to the highest percentage of stage transition in contemplators, whereas the condition offering self-efficacy enhancing information only led to the highest percentage stage transition in precontemplators. The findings from Chapter 6 further supported the distinction between contemplators and preparers: contemplators benefitted most from a combination of outcome information and self-efficacy enhancing information whereas preparers benefitted most from self-efficacy enhancing information only.

In conclusion, the data on the psychological differences between smokers in the present stages, the predicive validity of the stages and the differential effect of different sorts of information in the stages seem to support the four-stage model, although the distinction between precontemplators and contemplators needs further verification.

\section{Chapter 4: The Self-Evaluation Motivation model}

The main goal of Chapter 4 was to study the cognitive determinants of readiness to change and smoking behavior from another angle. That is, the data were not structured by stages of readiness to change but a continuous measure of intention to change was used instead. Furthermore, whereas both former chapters only reported on the univariate relations of the cognitive determinants of readiness to change and smoking behavior, this study aimed at exposing the multivariate relations. Lastly, in this study the focus was on the influence of outcome expectations on intention and behavior, whereas self-efficacy expectations were excluded from the analyses.

The Self-Evaluation Motivation model depicted physical, social and selfevaluative outcome expectations, two self-evaluation inhibiting processes (i.e. dissonance-reducing cognitions and attentional change processes), personal stanclards and their influence on intention to quit and quitting behavior. The results of multivariate regression analyses revealed the following relations.

First, the influence of physical and social outcomes and of dissonance-reducing cognitions and attentional change processes on intention and behavior, was mediated by self-evaluation. Second, perceived long-term health outcomes were not related to self-evaluation when smokers used few attentional change processes. Third, the inlluence of dissonance-reducing cognitions and attentional change processes on self-evaluation was partly mediated by their influence on physical and social outcome expectations. Thus, the model proposed a central role of negative self-evaluation in motivation to change. This might mean that self-help smoking cessation interventions should increase smokers' negative selfevaluation. According to Gibbons (1990), it is the awareness of discrepancy between the ideal self (personal standard) and the real self (being a smoker) that causes self-evaluation. Awareness of discrepancy might be brought about by self-observation, which, according to Bandura (1986) is a self-diagnostic device. Thus, an intervention would have to lead to enhanced self-observation. However, 
according to Gibbons (1990), awareness of discrepancy can make people either change their behavior (i.e. quit smoking) or do something to distract the attention from the self. Distraction from the self may be accomplished by what Beck et al. (1993) call a "cognitive blockade" or what Janis \& Mann (1977) call a process of "boistering". Thus, an intervention should not only increase selfobservation, but should also encourage smokers to change their behavior instead of distract the attention from the self. How could this be accomplished? Selfobservation may be increased by an intervention containing personal and recognizable feedback (i.e. tailored intervention). Furthermore, smokers might be provided with information which is thought to bring the solution, behavior change, within their reach. Information on cognitive and behavioral coping skills could be offered to increase the chances that the behavioral solution rather than the cognitive distraction solution is chosen to resolve the discrepancy.

In conclusion, this study showed that self-evaluation is a central determinant of intention to quit and actual quitting. Hence, smoking cessation interventions should increase self-evaluation. Furthermore, the two additional cognitive factors which were added (i.e. dissonance-reducing cognitions and attentional change processes) had the expected relations with physical and social outcome expectations and self-evaluation.

\section{The tailored interventions}

\section{The content of the tailored interventions}

In this section the considerations with regard to the development of the content of the tested tailored interventions will be discussed. From the three studies discussed above, we might conclude that a smoking cessation intervention should contain information aimed at; 1) increasing the perception of the physical and social positive outcomes of quitting; 2) increasing negative self-evaluation by inducing self-observation; 3) moderating the negatiwe outcomes of quitting; 4) increasing positive self-efficacy expectations to cope with social and emotional situations. Chapter 5 outlined how these elements could be incorporated in tailored interventions.

Earlier research suggested that interventions directed at different stages need to contain different sorts of information. However, the study in Chapter 3 did not provide strong support for the need for stage-matched interventions in the four early stages (immotives, precontemplators, contemplators and preparers). That is, there was a linear relation between readiness to change and increases in positive outcome expectations and self-efficacy. Furthermore, no decisive data from a critical experiment on stage-matched information were available. Therefore, the hypotheses based on the $\varnothing$-pattern of Chapter 2 were tested in the study in Chapter 6 and the expectations were extrapolated to the stage model with four instead of three early stages. With regard to both extreme groups, immotives and preparers, the expectations were as follows: immotives would benefit most from outcome information only, whereas self-efficacy enhancing 
information might even work detrimentally by triggering defensive information processing. That is, the strong behavior changing appeal of the latter sort of information is largely mismatched with the low readiness to quit of immotives. Preparers would benefit most from self-efficacy enhancing information, whereas outcome information might be redundant and therefore inhibit information processing. It was expected that smokers in the intermediate groups, precontemplators and contemplators, would benefit most from the combination of both sorts of information, whereas one sort of information might not be enough to stimulate stage-transition.

The study in Chapter 6 showed that, compared to the no-information control condition, immotives benefitted most from an intervention containing outcome information, whether this was in combination with self-efficacy enhancing information or not. Precontemplators benefitted most from self-efficacy enhancing information, whether this was in combination with outcome information or not. Thus, there was one intervention that was significantly more effective than no information in both stages: the intervention containing outcome information as well as self-efficacy enhancing information. Contemplators benefitted most from the combination of both sorts of information, whereas one sort was not enough. Preparers benefitted most from self-efficacy enhancing information, whereas an intervention containing outcome information had little effect.

\section{The effectiveness of the tailored interventions}

The results with regard to the effectiveness of the taillored interventions will be structured according to the five treatment evaluation strategies proposed by Kazdin (1986). The research questions and the results of the studies presented in the effectiveness studies in Chapter 6 to 9 are summarized in Table 10.1 and 10.2 .

In the treatment package strategy, interventions are compared to no intervention. The study in Chapter 6 showed that the single tailored interventions were more effective than no information at three months follow-up (research question 1). Only with regard to the seven-day quit criterion was the tailored intervention containing outcome information only no more effective than no information. After fourteen months, all three interventions led to more retrospectively reported 24 -hour quit attempts while the intervention containing both sorts of information led to more continuous abstinence, compared to no information (research question 6). These results were found in a sample with varying readiness to change.

In the sample with low readiness to change $(78 \%$ immotives and $22 \%$ precontemplators), the multiple tailored intervention and the single tailored intervention were more effective than no information whereas the 46-page selfhelp guide was not (research question 10). However, this effect was only found in immotives; in precontemplators none of the three self-help intervention was more effective than no information. This latter finding seemed especially due to 


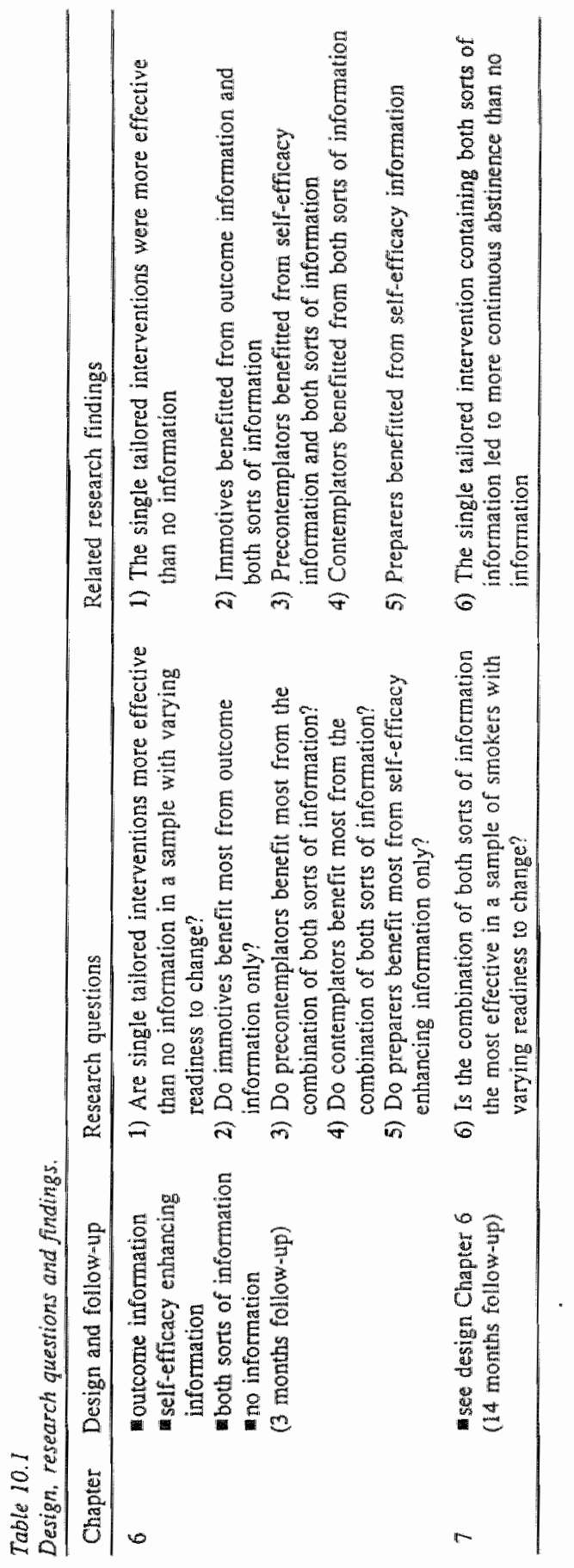




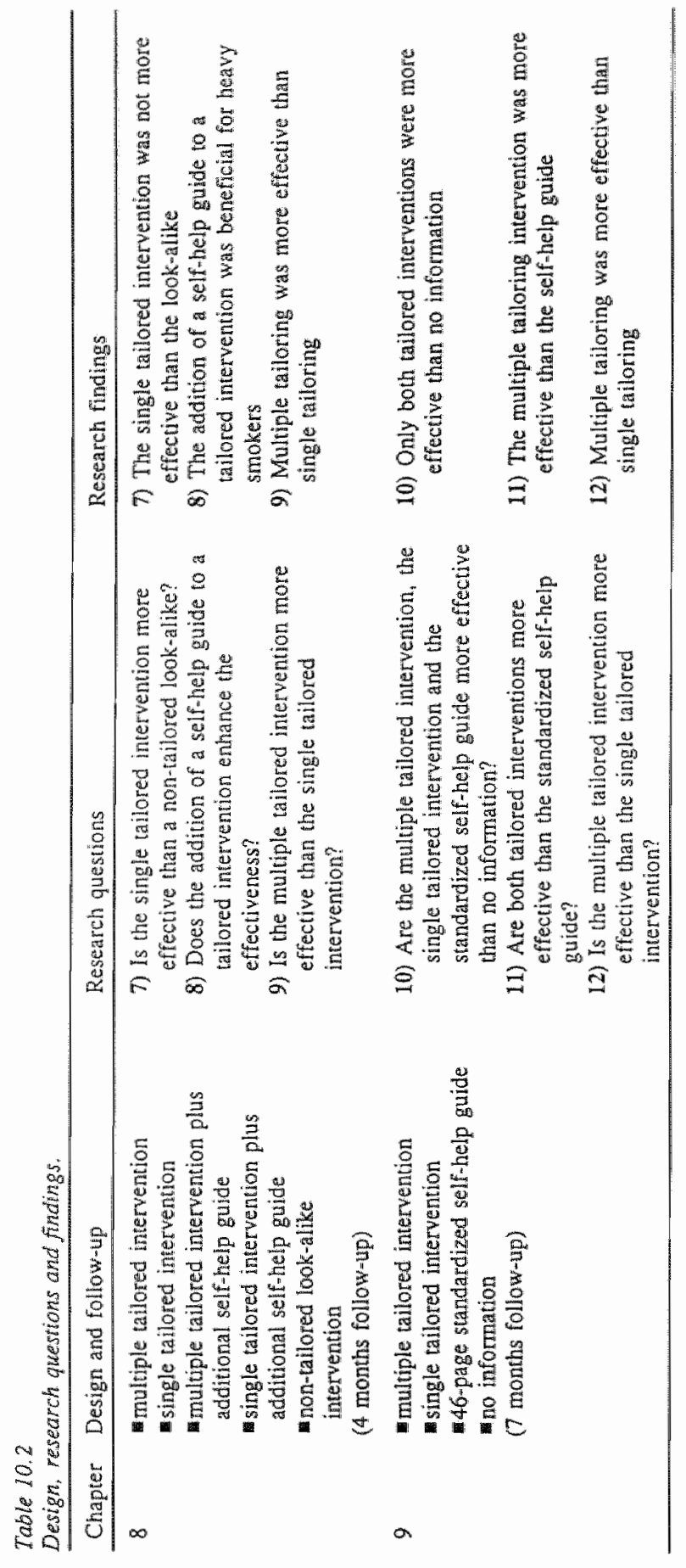


the high percentage of stage transition - $40 \%$ - in the no-information control group after seven months. The single tallored condition led to $35 \%$ stage transition. In the study in Chapter 6 , the percentages of stage transition in precontemplators in the no-information control group and the single tailored intervention were about $17 \%$ and $35 \%$, respectively, after three months. Combining these findings, it seems that the percentage of stage transition in the no-information control group increased from three to seven months after posttest, whereas the percentage in the tailored condition was stable. Tentatively, this pattern might be interpreted as showing that the tailored intervention in precontemplators only hastened the process in which they were already engaged, in contrast with immotives for whom the tailored intervention did have a surplus value, compared to no information. As suggested by Curry, McBride, Grothaus, Louie \& Wagner (1995), who also found a high percentage of quitting activity in their control group, the effect might be brought about by the social pressure smokers perceive at the moment they join a study on smoking cessation and fill out smoking cessation questionnaires. This effect of social pressure must thus have been somewhat delayed in our study, as it was not yet present at the three months follow-up. This suggestion clearly has to be tested in future research.

The results of the stratified analyses (research questions 2 to 5 ) can be summarized as follows: depending on the stage of readiness to change, a single tailored intervention containing a specific sort of information was more effective than no intormation.

In conclusion, the treatment package strategy revealed that a single tailored intervention was more effective than no information after three and after fourteen months. With regard to the content of the intervention, it might be concluded that the intervention containing a combination of outcome and selfefficacy enhancing information was effective in smokers with low readiness to change and in a population with varying readiness to change.

In the dismantling treatment strategy, components of a given treatment package are isolated. In the present studies, the treatment package consisted of a tailored intervention containing a combination of outcome and selfefficacy enhancing information. In the study in Chapter 6 , the effects of this package were compared to the effects of both isolated components (research questions 2 to 5). First, the experimental conditions were compared to the no-information control condition. The results showed that the effects differed per stage, as was reported above. However, the fact that one sort of information is significantly more effective than no information whereas the other sont of information is not, does not implicate that both sorts of information differ significantly. Second, owing to the above a more conclusive test was conducted which compared the experimental conditions directly to each other. The results showed that only two of these many contrasts were significant, although all were in the expected direction: contemplators who received both sorts of information had a significantly higher intention than those who received self-efficacy enhancing information, while preparers who received self-efficacy enhancing information quit significantly more often for the last seven days than those who received 
outcome information. Third, a match-mismatch test was conducted in which smokers who were assigned to a matched treatment were compared to smokers who were assigned to a mismatched treatment. This test supported the above results in contemplators and preparers, but not in immotives and precontemplators. However, the match-mismatch tests are less easy to interpret in terms of effectiveness of isolated components because smokers in the matched or mismatched conditions are pooled. In conclusion, the effectiveness of components depended on the stage of readiness to change.

In the constructive treatment strategy, components are added to an intervention. First, the study in Chapter 8 showed that adding the self-help guide to a tailored intervention led to higher effectiveness in highly dependent smokers (research question 8 ). The effect of the additional self-help guide could be brought about by the more extensive information on different sorts of cognitive and behavioral skills to cope with the urge to smoke.

The study in Chapter 8 also showed that tailored information (i.e. content information with the addition of a 'tailoring' component) was not more effective than the standardized look-alike in smokers with low readiness (research question 7). As no control group was used in the study in Chapter 8, it is not clear whether this finding can be attributed to a possible large effect of the nontailored look-alike.

In conclusion, the latter null-finding might mean that the proposed tailoring working mechanisms, adaptation of the content information and feedback, have little surplus value among smokers with low readiness to change. However, replication is needed to confirm this supposition.

In the parametric treatment strategy, existing components in an intervention are expanded quantitatively. The studies in Chapters 8 and 9 showed that the multiple tailored intervention was more effective than the single tailored intervention (research questions 9 and 12). However, in both study designs the specific 'intervention elements' which were responsible for this effect of multiple tailoring could not be identified. For example, the effect of multiple tailoring might be caused by the fact that the information on the pros and cons of quitting and on the use of skills to quit was offered more than once. However, we do not know to what extent the tailoring of this information was essential. Three consecutive and related, but non-tailored letters might have similar efficacy. Furthermore, we do not know to what extent the three assessments - one mailed questionnaire and two additional telephone interviews contributed to the efficacy of multiple tailoring. Finally, the larger effect might be caused by the ipsative feedback. In conclusion, tripling the number of interventions (multiple versus single) increased the effectiveness.

In the comparative treatment strategy, existing treatments are compared. In the study in Chapter 9, three independent interventions were compared (research questions 11 and 12): the multiple tailored interwention, the single tailored intervention and the standardized self-help guide. The multiple tailored intervention was more effective than the single tailored intervention and the selfhelp guide, although the single tailored intervention was not more effective than 
the standardized guide.

In conclusion, the multiple tailored intervention was clearly the most effective in smokers with low readiness to quit.

\section{Summary}

The present tailored interventions all met the minimal criterion: they were more effective than no intervention. This was the case for smokers in all four stages, while the sorts of information which were effective depended on the stage of readiness to change. In smokers with low readiness to quit, the single tailored intervention was not more effective than its non-tailored look-alike and the addition of the standardized self-help guide was only beneficial to highly dependent smokers. Furthermore, in precontemplators, none of the self-help interventions was effective, in contrast with the clear effects in immotives. Finally, in smokers with low readiness to change, the multiple tailored intervention was more effective than the single tailored intervention and the standardized self-help guide. For smokers with low readiness to change, the single tailored intervention would be the intervention of choice above the selfhelp guide.

\section{Methodological issues}

The results in this thesis need to be interpreted against the background of the soundness of the methodology used. The first issue discussed below concerns the possible biasing effects of the different item contents of the scales measuring outcome and self-efficacy expectations across the studies. The second issue deals with the generalizability of the results, given the specifically recruited samples of smokers. The third issue focuses on the newly developed outcome measure; stage transition.

\section{Operationalization and measurement of the psychological constructs}

In the different studies presented, the outcome and self-efficacy expectations were assessed using the same formulations. The outcome expectation items were formulated as follows: "If $\mathbb{I}$ quit smoking, then......". Perceived self-eflicacy, except in the Chapter 2 study, was assessed as follows: "Do you think you are able to refrain from smoking in situation ...?". Thus, on the item level the constructs were mostly assessed with similar formulations. However, the number and sort of items comprising the scales measuring the outcome and self-efficacy expectations differed across the studies (Table 10.3). This can be explained as follows. The items from the Chapter 2 study were gathered from: 1) the literature; 2) smoker focus groups; 3) theoretical considerations; 4) expert opinions. After this exploratory study, the items included in the questionnaires were determined by: 1) psychometric considerations (such as reliability and expected relations with other wariables); 2) theoretical denands (items must be 
included that represent, for example, the expected physical, social and selfevaluative outcome factors); 3) the fact that most items were also used as the basis for the tailored interventions. Because of the limited space in the questionnaires used in the study in Chapter 6, it was decided to include only those negative outcome expectation items that were used in the tailoring system. Unfortunately, this resulted in low reliability $(\alpha=57)$. Because the large item set from the first study had not yet been tested longitudinally in the study in Chapter 8 , most items from the study in Chapter 2 were included again. In the study in Chapter 9, the resulting final short and reliable questionnaires were composed: 12 items (four factors) assessing the pros of quitting, 10 items (one factor) assessing the cons of quitting and 12 items (three factors) assessing selfefficacy.

Table 10.3

Number of items in the scales assessing the pros and cons of quiting and selfefficacy in the differen studies, Cronbach alpha reliabilities and mean inter-item correlations.

\begin{tabular}{llllllllll}
\hline Chapter & & pros & \multicolumn{3}{c}{ cons } & \multicolumn{3}{c}{ selfwefficacy } \\
\hline & items & $\alpha$ & $\mathrm{r}(\mathrm{i}-\mathrm{i})$ & items & $\alpha$ & $\mathrm{r}(\mathrm{i}-\mathrm{i})$ & items & $\alpha$ & $\mathrm{r}(\mathrm{i}-\mathrm{i})$ \\
2 & 25 & .89 & .25 & 10 & .73 & .21 & 25 & .97 & .46 \\
6,7 & 15 & .87 & .31 & 6 & .57 & .18 & $4 / 3^{*}$ & $.81 / .86^{*}$ & $.69 / .52$ \\
8 & 23 & .90 & .28 & 10 & .82 & .31 & 13 & .94 & .55 \\
9 & 12 & .87 & .37 & 10 & .84 & .34 & 12 & .94 & .57 \\
\hline
\end{tabular}

Note. $r(i-i)=$ mean inter-item correlation. *Two scales were used to assess self-efficacy: coping with emotions and coping with social situations.

As can be seen in Table 10.3, the Cronbach alpha $(\alpha)$ reliabilities of the scales were satisfactory, ranging from .73 to .97 , apart from the reliability of the negative outcome expectations scale $(\alpha=.57)$ in the study in Chapter 6 . The low reliability of the negative outcome expectations scale may be partly due to the few items in the scale. Furthermore, the low mean inter-item correlation (.18) indicates the scale is internally less consistent: the items seem to refer to the same psychological construct to a lesser degree.

Although we can not rulle out that the scales containing different items measure slightly different constructs, it might be argued that the scales had more similarities than differences.

First, the scales assessing the positive outcome expectations in the different studies all contained items on the long-term physical outcomes, short-term physical outcomes, social outcomes and self-evaluative outcomes. Second, the scales assessing self-efficacy in the different studies all contained items on coping with emotions and social situations. With regard to the negative outcome expectations, the study in Chapter 2 contained items with regard to the loss of the smoker's image. After this study, these items were removed from the scale because they had low discriminative power with regard to the stages of change. 
In the studies presented in Chapters 8 and 9 , these items were replaced by the more powerful items assessing the anticipation of the loss of means to cope with negative emotions. Hence, the scales assessing the positive outcome expectations and self-efficacy expectations were considered to measure the same phenomena over the studies, whereas it might be argued that the scales assessing the negative outcome expectations assessed the construct somewhat less consistently.

\section{Recruitment of participants.}

The participants were all recruited reactively using advertisements in local newspapers throughout the Netherlands. It might be argued that this recruitment strategy has led to a select sample of smokers, thereby limiting the generalizability of the results. However, the following considerations moderate this criticism.

First, in the advertisements for the effectiveness studies, participants were offered the opportunity to win a bonus prize amounting to $\$ 100$. It might be argued that using such an extrinsic motivator instills a motive to join the study which is independent of the involvement of the smoker in smoking or smoking cessation. In other words, offering monetary incentives might lead to a less biased sample of smokers.

Second, in all studies, specific measures were taken to specifically attract smokers with low readiness to quit. In the advertisements the target group was defined as: "Smokers who are not planning to quit within the next six months, or may be planning to never quit". Furthermore, it was stated explicitly that smokers did not have to quit to join in the present study. This framing was expected to also lead to the recruitment of smokers with high resistance to change their smoking behavior and with explicit low readiness to quit. Qualitative findings from hundreds of conversations with smokers who registered during recruitment periods support this notion: many of them angrily objected to the notion of smoking cessation and the social pressure to quit which they encountered in their lives.

Finally, the extent to which the present results can be generalized depends on the populations we wish to target in the future. That is, if the population we wish to target in the future is also recruited by advertisements, no generalizability problems exist. The present results probably tell us about the effectiveness of tailored interventions in smokers who read a newspaper advertisement about smoking and smoking cessation and feel encouraged to phone up for more information partly because they do not feel coerced to change. In future applications of tailoring this might well be the sample that is targeted.

Concluding, although the samples are selective, the results with regard to the cognitive determinants and the effectiveness of the tailored interventions are considered to be largely generalizable to the Dutch smokers in general. Furthermore, the generalizability with regard to future applications of tailored interventions in which smokers are recruited reactively is considered to be good. 


\section{Stage trunsition}

Because the primary target group consisted of smokers who were not planning to quit within the next six months, the behavioral outcome measure seven-day quit was considered to be less appropriate. In the study in Chapter 8, the experimental conditions led to percentages of seven-day quitting ranging from $0.8 \%$ to $7.4 \%$, while in the study in Chapter 9 it only ranged from $3.2 \%$ to $5.5 \%$. Particularly in the latter case, the conditions seemed to hardly lead to differential effects in smokers with low motivation when using the seven-day quit measure. Velicer, Prochaska, Rossi \& Snow (1992) argue that such a sevenday quit point prevalence measure does." ".... not reflect the complex variety of changes that are involved in smoking cessation" (p. 35). Therefore, stage transition was used as the primary outcome measure.

First, in the stages of change model, stage transition is an appropriate and relevant outcome measure for people in all stages. Second, because stage of readiness to change is a strong predictor of quitting behavior (Chapter 3; DiClemente et al., 1991), forward stage transition was considered to be a relevant and desirable motiwational/cognitive or behavioral change. Third, the data from the study in Chapter 6 further supported the use of stage transition: compared to the stage transition outcome measure, intention to quit revealed about the same pattern of effects in immotives, precontemplators and contemplators and the seven-days quit measure revealed the same pattern in preparers. In fact, the stage transition outcome measure combined both conventional outcome measures: the early stages were defined in terms similar to a measure of intention, whereas the later stage (i.e. the action stage) was defined in terms of behavior.

Stage transition was assessed by subtracting the pre-test stage score from the post-test stage score. Stages were coded as follows: immotives (1), precontemplators (2), contemplators (3), preparers (4), ex-smokers in action (5). If the difference between pre- and post-test was larger than 0 , the smoker had made a forward stage transition. Immotives could transit to one of four subsequent stages, precontemplators to three subsequent stages, and so on. It follows from this that forward stage transition in immotives may be greatly different from forward stage transition in, for example, preparers. This notion was supported by additional analyses on the data presented in Chapter 6, which have been published elsewhere (Dijkstra, De Vries, Roijackers \& Van Breukelen, 1997; Dijkstra, De Vries, Roijackers \& Mudde, 1997). These analyses showed that in immotives, changes in perceived positive outcome expectations between $\mathrm{T} /$ and $\mathrm{T} 2$ were predictive of stage transition at T3, whereas in preparers, changes in self-efficacy with regard to coping with negative emotions were predictive of stage transition at T3. De Vries and Mudde (in press) reported similar results. On the other hand, the percentages of stage transition in immotives, precontemplators, contemplators and preparers were $30 \%, 35 \%, 33 \%$ and $45 \%$, respectively. "The latter two percentages differed only marginally $(p<10)$. Thus, it seems that smokers in the different stages do not differ in the 
ease with which they make a forward stage transition. Indeed, there seems to be no a priori reason why smokers in the different stages would have more difficulty in making a forward stage transition. In conclusion, the primary outcome measure stage transition is a useful outcome measure in research in smokers with low readiness to quit and in general in research using the stages of change construct.

Using forward stage transition as the primary outcome measure in the present effectiveness studies, implies that interventions were expected to influence forward stage transition. It could be argued that interventions also influence backward stage transition. For example, a smoker in contenplation might postpone quitting for different reasons and transit backward to precontemplation or immotivation. Additional analysis on the data in Chapter 6 revealed that a backward stage transition measure (backward transition (1) versus no backward transition (0)) revealed results which were partly unexpected, hard to interpret and undifferentiated (Dijkstra, De Vries, Roijackers \& Van Breukelen, 1997). For example, in contrast with the forward transition data, the three experimental conditions in the Chapter 6 study led to significantly fewer precontemplators moving backward, compared to the control group, whereas in contemplators and preparers, none of the experimental conditions led to significantly different percentages of backward stage transition. Future research will have to address the applicability and validity of backward transition as an outcome measure (see also De Vries \& Mudde, in press).

All three experimental studies showed significant differences between the conditions with regard to forward stage transition. The percentages of stage transition in the experimental conditions in the study in Chapter 6, ranged from $27.5 \%$ to $54.3 \%$. Furthermore, the matched intervention conditions roughly led to a doubling of the percentages compared to no information. For example, whereas among precontemplators about $19 \%$ moved spontaneously forward through the stages, a tailored intervention led to over $40 \%$ of stage transition. In the study in Chapter 8 , the multiple tailored intervention nearly doubled the percentage compared to the standardized condition (35\% versus 19\%) and in the study in Chapter 9 , the multiple tailored intervention even tripled the percentage compared to no intervention, in immotives ( $42 \%$ versus $14 \%$ ). In conclusion, the findings with the stage transition measure were more differentiated than the findings with the seven-day quit measure. The stage transition measure might be considered to be more sensitive to changes in the process of smoking cessation. 


\section{Applications of the findings}

\section{A typology of smokers in four stages}

How can we apply the results from the present studies to counseling smokers with low readiness to change and developing self-help materials for this group? In encouraging these smokers to quit, it is of importance to realize that they have a long way to go. They have to move through the contemplation and preparation stage in order to make a serious quit attempt and to end up in the action stage. Thus, smokers with low readiness need to be guided through these stages. In the following section, a typology of smokers in the four stages will be outlined and stage-specific goals and action will be suggested. Because the present thesis was not concerned with relapse, the transition from action to maintenance will not be addressed.

The present typology of smokers in the four stages is based on the findings using the transtheoretical model (Prochaska, DiClemente \& Norcross, 1992), the results presented in this thesis and on additional statistical analyses on the data in Chapter 6 which have been presented elsewhere (Dijkstra, De Vries, Roijackers \& Mudde, 1997; Dijkstra, De Vries, Roijackers \& Van Breukelen, 1997). These additional analyses showed to what extent cognitive changes between $\mathrm{T} 1$ and $\mathrm{T} 2$ were predictive of forward stage transition from baseline, assessed at T3. The analyses resulted in the following findings. In inmotives, a significant increase in the perceived pros of quitting was predictive of stage transition; in precontemplators a significant increase in self-efficacy to cope with social situations was prediclive of stage transition; in contemplators a borderline significant increase in self-efficacy to cope with negative emotions was predictive of forward stage transition and in preparers a significant increase in self-efficacy to cope with negative emotions was predictive of forward stage transition. These findings show what changes in cognitions have to be brought about and, hence, what sorts of information smokers in different stages need.

In communicating the different sorts of information, the principles of motivational interviewing (Miller \& Rollnick, 1991) are of basic importance. These principles can be applied in individual counseling situations as well as in self-help materials and they were explicitly included in the tailored interventions which were tested in this thesis. Miller and Rollnick (1991) give five general principles underlying motivational interviewing: expressing empathy, avoiding argumentation, rolling with resistance, developing discrepancy and supporting self-efficacy. Developing discrepancy might be applied by communicating information on the negative consequences of smoking (or their complements; the pros of quitting) and increasing the awareness of cognitive dissonance. Selfefficacy might be supported by offering skills to cope with the urge to smoke in the case of a quit attempt. These two principles were investigated and supported in the present thesis. The remaining three principles - expressing empathy, avoiding argumentation, rolling with resistance - are communicational and characterized by acceptance and soft confrontation. The underlying attitude 
derives from the understanding that the client's situation can be understood:.... "as one of being "stuck" through understandable psychological principles" (Miller \& Rollnick, 1991, p. 56). Thus, as long as smokers have not yet committed themselves to a decision to actually quit, it is essential to integrate these three principles in the intervention.

Finally, the present typology is based on qualitative findings from telephone conversations with smokers who registered to join in one of the studies and from experiences as a therapist in cognitive/behavioral group therapy for smoking cessation. First, for each of the four stages it will be described how smokers in these stages can be recognized. Second, the stage-specific goals will be defined and, third, recommendations with regard to communicating the specific sorts of information will be given.

\section{Immotives}

Immotives may be recognized by their striking lack of negative self-evaluation; they portray themselves as satisfied with their smoking behavior. On the other hand, they easily become defensive towards behavior changing appeals and in today's society they often feel discriminated against as smokers.

Immotives have no concrete plans to quit or they even have plans to continue smoking, whereas precontemplators have concrete, though long-term, plans to quit. The goal in immotives, who are considered "to be stuck" in low readiness, is to move to precontemplation, that is, to be "underway to contemplation". The study in Chapter 4 showed that the largest difference in negative self-evaluation was between immotives and precontemplators. Hence, immotives must start to evaluate themselves negatively, with regard to their smoking behavior in order to move to the precontemplation stage. This might be accomplished by motivating them to process information on the negative health and social consequences of smoking or, viewed differently, on all the sorts of pros of quitting. The additional analyses (Dijkstra, De Vries, Roijackers \& Mudde, 1997; Dijkstra, De Vries, Roijackers \& Van Breukelen, 1997) also showed that an increase in the perceived pros of quitting may encourage them to move forward.

Immotives have few motives to process information on the pros of quitting. Because they do not request information on smoking cessation, any possible occasion to offer such information must be exploited, such as, if they enter the health care system. For instance, dentists, pharmacists and general practitioners can briefly offer the relevant information, using the communicating principles of motivational interviewing. Furthermore, health professionals must be patient. Smokers can not be forced to quit and particularly immotives may take a long time to move forward. The health educator must offer the right information framed in the right way and if possible plan a follow-up, for example by planning to go into the subject again the next time he or she meets the smoker professionally. 


\section{Precontemplators}

Precontemplators may be recognized by their relative awareness of the negative consequences of their smoking and their subsequent negative self-evaluation in combination with a contrasting lack of a short-term plan to quit. Whereas contemplators may say; "I am aware of the negative consequences of smoking and that is why I plan to quit within the next six months", precontemplators are to a certain degree aware of these consequences but they still may plan to keep on smoking for years.

The goal with precontemplators is to move them to contemplation. Precontemplators must be encouraged to contemplate quitting. That is, instead of adhering to their long-term quitting plan, they must be stimulated to think about their quitting plan again and integrate the knowledge of the pros of quiting they have. Compared to smokers in the adjacent stages, they find quiting more difficult than immotives and have, compared to contemplators, low confidence in being able to quit. Moreover, from the additional analyses (Dijkstra, De Vries, Roijackers \& Mudde, 1997; Dijkstra, De Vries, Roijackers \& Van Breukelen, 1997) it follows that they need to increase their self-efficacy to cope with social situations. Such an increase might lead to the availability of the positive outcomes they already anticipated.

Precomplators already know that there are many potential reasons to quit and, hence, reasons to further process information on smoking cessation. This fact could be fed back to the smoker. Besides using the principles of motivational interviewing, it is important to frame the self-efficacy enhancing information according to their long-term plan to quit. That is, the self-efficacy enhancing information is not meant to support an actual quit attempt but to motivate precontemplators by increasing their experience of control. Once they realize that smoking cessation may be a realistic personal goal, they may move forward to contemplation. Again, realistic expectations from the health educator and follow-ups are important in the guidance of these smokers.

\section{Contemplators}

Contemplators may be recognized by their high awareness of the negative consequences of their smoking and by strong negative self-evaluation and a contrasting lack of a commitment to a decision to quit. They may be explicit about the positive outcomes they anticipate from quitting but they seem to postpone an actual quit attempt.

The goal in contemplators is to move them to preparation. Contemplators must be encouraged to no longer postpone quitting but make a commitment to a decision to quit in the short-term. From the study in Chapter 6 it follows that they need information on the pasitive outcomes of quitting combined with selfefficacy enthancing information. With regard to their strong negative selfevaluation, they must be encouraged to choose the behavioral solution, that is quitting, rather than the cognitive. This could be brought about by self-efficacy 
enhancing information. The additional analyses (Dijkstra, De Vries, Roijackers \& Mudde, 1997; Dijkstra, De Vries, Roijackers \& Van Breukelen, 1997) show that they specifically need an increase in self-efficacy to cope with negative emotions.

Although contemplators may seem to have high readiness to change, the communicating principles of motivational interviewing must be applied. Contemplators have high levels of negative self-evaluation and they may react defensively to information which further confronts them with the negative consequences of their smoking behavior. By helping them to perceive quitting as a realistic solution of their experienced discrepancy, they may move to preparation. The self-efficacy enhancing information may be partly motivational and partly to already prepare an actual quit attempt and the health educator can gently use a coaching attitude to support the fragile quitting plan. Contemplators may move rapidly to the preparation stage, but may subsequently regress easily to contemplation. A follow-up appointment must be made explicitly without expressing expectations which are too high.

\section{Preparers}

Preparers may be recognized by their changing smoking behavior and their need to enumerate even the slightest possible positive outcomes of quitting. They have made a commitment with a decision to quit in the short-term and they may be very explicit in their dissatisfaction with their smoking behavior.

The goal with preparers is to move them to action. Preparers must be encouraged to plan the actual quit attempt. Their main tasks are to pick a quitting day and to plan and execute the necessary preparations. Furthermore, they specifically have to prepare the first days of their quit attempt. As indicated by the additional analyses (Dijkstra, De Vries, Roijackers \& Mudde, 1997; Dijkstra, De Vries, Roijackers \& Van Breukelen, 1997), an increase in selfefficacy to cope with negative emotions will increase the chances that they will indeed engage in a serious quit attempt. Chapter 7 showed that high self-efficacy to cope with negative emotions is important to maintain quitting.

Preparers can be coached. They can be supported in planning the actual quit attempt and talked through the possible barriers they can encounter. It is important that the health professional offers his of her expertise but that the smoker is the "owner" of the solutions. Ideally, a quitting day is planned and follow-up appointments must be planned jointly in the first days of quitting. Relapse is common in smoking cessation and, consequently, so is regression to earlier stages is. Many preparers, however, can be coached to keep on changing their smoking behavior and to plan another quit attempt.

\section{Using the typology}

This fypology of the smokers in the four early stages must be used with care. The data on the cognitive differences between smokers in the different stages, 
the predictive value of the stages and the effectiveness of stage-matched information can only be applied to groups of smokers, not to individuals. The typology has only a probabilistic relation with individual states and future behavior. Thus, for example, not all smokers who are classified as immotives according to the presently used algorithms have low negative selfevaluation and not all smokers in preparation need only self-efficacy enhancing information. The algorithms are meant to refer to an underlying dimension of readiness to change. Interventions have to take into account this dimension. The algorithms are only one way, though an important one, to assess an individual's readiness to change. In diagnosing an individual's readiness to quit, the salience of the expected outcomes, the commitment to the quitting plan and the use of attentional change processes may be of importance. Computer-tailored interventions are a promising means to take into account a complex configuration of individual features.

\section{The application of computer-tailored interventions}

The presently developed tailoring systems producing tailored interventions are ready to be implemented in the health care system. Once the computer hardware is available and the software is installed, the individual data can be fed in and the tailored interventions printed out. The tailored interventions can then be mailed to the individual. Before that, an individual has to fill in the tailoring questionnaire, which, first, has to be distributed to the smoker. The distribution of tailoring questionnaires, when possible in combination with a pre-paid return envelope, can be organized in many ways. For example, pharmacies, out-patient clinics, dentists, can all offer the tailoring questionnaire to smokers. They do not all have to possess the computer hard-and software. Regional municipal health services may serve as the regional initiators and organizers of the implementation of the tailored interventions in the health care system. For example, they may provide tailoring questionnaires to several regional distribution points. Smokers who have filled in the questionnaire may send their tailoring questionnaire to the regional municipal health service where the individual data are processed to individual interventions and mailed back. In this way, the tailored intervention can be an attractive and effective extra service for clients or patients who visit the questionnaire distribution points. Furthermore, the tailored interventions might be used as an extension of the professional advice of, for example, the general practitioner (GP). The GP could advice each smoker or only smokers who have smoking-related health complaints, to fill in the tailoring questionnaire.

The implementation of the more powerful multiple tailored intervention needs some more organization, given the two additional telephone assessments needed for the second and third tailored intervention letter. However, the telephone calls only have to be planned as soon as the first tailored letter is mailed to the individual. Furthemore, the telephone calls are not counseling calls and so the telephone operators need no additional training. The first assessment is with a 
tailoring questionnaire which can be distributed through the health care channels mentioned above. Thus, although the multiple tailored intervention needs more organization, it is a powerful intervention which is highly effective in smokers with low readiness to change.

\section{Recommendations}

The present thesis reports on a line of studies in the framework of the development of computer-tailored interventions in smoking cessation. Although many questions have been answered, other questions remain or have arisen. Therefore, in this section recommendations for future research will be given with regard to the cognitive determinants, the stages of change and the effectiveness of tailored interventions.

\section{Cognitive determinants and the stages of change}

This thesis reported on physical, social and self-evaluative outcome expectations and self-efficacy with regard to social and emotional tasks. However, the differential function and predictive power of these factors needs further study. For example, it is of interest why and to what extent changes in certain factors are related to forward stage transition and quitting behavior. Furthermore, future studies could investigate to what extent the different factors and their predictive power differ for subgroups of smokers. For example, the predictive power of the perceived short-term health outcomes of smoking might be different in young smokers compared to older smokers and the importance of coping with social situations in smoking cessation might differ for males and females.

With regard to the Self-Evaluation Motivation model (SEM), the extent to which it applies to other behaviors could be studied. Furthermore, it is of importance to study the relation between self-evaluative outcome expectations and self-efficacy expectations. The two cognitive factors introduced in the SEM - dissonance-reducing cognitions (DRC) and attentional change processes (ACP) - need further investigation. For example, experimental studies could, first, identify ways to change the DRCs and assess whether changes in DRCs lead to higher motivation to change. Second, the factors or sorts of information which instill an automatic and genuine attentional change process could be studied. Furthermore, the ACP measure might be validated using information processing measures. For example, it could be hypothesized that the higher a smoker scores on ACPs, the faster the domain-specific information is processed (i.e., information on smoking and smoking cessation). In a modified Stroop color naming task, smokers can be confronted with domain-relevant words and neutral or other control words in different colors on a computer sereen. They are instructed to name the color of the word as fast as possible while a voice level detector records the response latency (Lavy, 1993). It is expected that smokers who score high on ACPS will only be distracted more easily by the domainspecific words and, hence, will be slower in naming the color. Such a finding 
would validate the ACP self-report measure.

With regard to the stages of readiness in the present thesis many question remain. First, it is of importance to further investigate to what extent the stages refer to discrete states of psychological functioning. For example, the algorithm stage solution could be compared to the 32 -item readiness to change measure stage solution and a continuous intention measure. Moreover, a more discriminative description of smokers in the four stages is needed. For example, we would like to further explain how precontemplators and contemplators differ and why immotives did and precontemplators did not benefit from the self-help interventions in Chapter 9. Second, the issue of stage-matching intervention could be addressed. That is, in smokers with low readiness to change, none of the three sorts of information tested was more effective than another. Future studies could come up with alternative matching factors. For example, the framing of the information or the extensiveness of the self-help materials might be matched or mismatched. Third, the surplus value of tailoring information in the group of smokers with low readiness to change could be studied. The disappointing result in the study in Chapter 8 - the single tailored intervention was no more effective than its non-tailored look-alike in immotives and precontemplators - needs verification.

\section{Whether, why and for whom do tailored intervention work}

Three main questions are of importance with regard to the efficacy of tailored interventions. The first question is: "Do tailored interventions work (better than other existing self-help materials)". To answer this question, future studies will have to include a no-information control group or a condition with an existing intervention. In Kazdin's (1986) evaluation strategy structure these strategies are called the treatment package strategy and the comparative treatment strategy, respectively. The second question is: "Why do tailored interventions work?". To answer this question, Kazdin's (1986) dismantling, constructive and parametric strategy can be applied. The dismantling strategy - in which components of a treatment package are isolated - could be used as follows: with regard to tailored interventions in general, the proposed working mechanisms might be investigated by isolating them. For example, a tailored intervention which includes both sorts of working mechanisms, adaptation of the messages and feedback, might be compared to: 1) a tailored intervention with adapted messages only and; 2) a tailored intervention which is not adapted but does provide certain types of feedback. With regard to multiple tailoring, the components that might add to the efficacy could be tested separately. For example, the multiple tailored intervention might be compared to: 1) an assessment-only condition; 2) a multiple but non-tailored intervention plus assessment; 3) a multiple but nontailored intervention without the assessment; 4) an assessment and ipsative feedback only condition.

The constructive strategy - in which components are added to a treatment package - could be used as follows: first, the content of tailored interventions 
could be extended. For example, the currently developed tallored intervention contained information meant to increase the perception of the physical and social positive outcomes of quitting, increase negative self-evaluation by inducing selfobservation, moderate the negative outcomes of quitting and increase selfefficacy expectations to cope with social and emotional situations. This intervention might be compared to an intervention with the same content but with additional information. Many sorts of information could be added. For example, information meant to increase risk perception, instill a social norm or increase positive modeling influences. Second, tailored interventions could be complemented with other components, such as audio or video recordings, external incentives, a quitting contract or other self-help materials.

The parametric strategy - in which existing components in a treatment package are expanded quantitatively - could be used as follows: as most tailored interventions address skills to change a behavior, such interventions could be compared to interventions which address even more skills. Furthermore, in the currently developed tailoring system, several personal features were fed back, such as the name of the respondent, the number of cigarettes smokers a day, and so on. This intervention might be compared to an intervention including more such personal and recognizable features.

The third question with regard to effectiveness of tallored interventions is: "who do tailored interventions work for?". This question can be answered largely by searching for interactions between intervention conditions and different groups of smokers in data from efficacy studies. To be able to do this, the pretest questionnaire has to operationalize the subgroups under investigation. Furthermore, the two questions addressed above, "Do tailored interventions work" and "Why do they work", could be applied to subgroups.

Finally, it is important to study the dissemination of tailored interventions in the health care system. That is, the extent to which society will benefit from tailored smoking cessation interventions is partly a function of the extent to which the tailored interventions are distributed by the healih care system. The factors which influence the dissemination, such as motivation to distribute the tailored interventions and perceived barriers to distribute the tailored interventions, will have to be studied.

Concluding, many questions with regard to tailored interventions have not been answered yet. Answers will arise from experimental studies in the controlled laboratory environment as well as from field studies. The expenses and the efforts to investigate computer-tailored interventions are justified as long as they prove to be more effective in changing behavior than non-tailored intervertions. At present, it can only be concluded that tailored interventions hold great promise. 


\section{References}

Ajzen, I. (1988). Attitudes, Personality, and Behawior. Milton Keynes: Open University Press.

Ajzen, 1. (1991). The theory of planned behavior. Organizational Behavior and Human Decision Processes, 50, 179-211.

American Psychiatric Association (1994). Diagmostic and Statistical Mamual of Mental Disorders (4rd Ed.,rev.).

Bagozzi, R.P. (1992). The self-regulation of attitudes, intentions, and behavior. Social Psychology Qwarterly, 55, 178-204.

Bandura, A. (1986). Social Foundations of Thought and Action; A Social Cognitive Theory. Englewood Cliffs, NJ: Prentice-Hall.

Bandura, A. (1997). The Exercise of control. New York: Freeman.

Bandura, A., \& Cervone, D. (1983). Self-evaluative and self-efficacy meachanisms governing the motivational effects of goal systems. Journal of Consulting and Clinical Psychology, 45, 1017-1028.

Beck, A.T., Wright, F.D., Newman, C.F., \& Liese, B.S. (1993). Cognitive Therapy of Substance Abuse. New York: The Guilford Press.

Bertalanfy, L. von (1968). General System Theory; Foundations, Development, Applications. New York: George Braziller.

Blake, S.M., Klepp, K.I., Pechacek, T.F., Folsom, A.R., Luepker, R.V., Jacobs, D.R., \& Mittelmark, M.B. (1989). Differences in smoking cessation strategies between men and women. Addictive Behaviors, 14, 409-418.

Boyd, B., \& Wandersman, A. (1991). Predicting undergraduate condom use with the Fishbein \& Ajzen and the Triandis attitude-behavior model: Implications for public health interventions. Joumal of Applied Social Psychology, 21, $1810-1830$.

Brug, J. (1996). The Development and Impact of Compurer-Tailored Nutrition Education. Doctoral dissertation, Maastricht University, The Netherlands.

Brug, J., Glanz, K., Van Assema, P., \& Kok, G. (1996). Computer-tailored nutrition education: The impact of ipsative feedback. Manuscript submitted for publication.

Brug, J., Steenhuis, I., Van Assema, P., \& De Vries, H. (1996). The impact of a computer-tailored nutrition intervention. Preventive Medicine, 25, 236-242.

Brug, J., Steenhuis, I., Van Assema, P., Glanz, K., \& De Vries, H. (in press). Computer-tailored nutrition education: Differences between two interventions. Health Education Research.

Budd, R.J., \& Rollnick, S. (1996). The structure of the readiness to change questionnaire: A test of Prochaska \& DiClemente's transtheoretical model. British Joumal of Health Psychology, 1, 365-376.

Burling, T.A., Marotta, J., Gonzalez, R., Moltzen, J.O., Eng, A.M., Schmidt, G.A., Welch, R.L., Ziff, D.C., \& Reilly, P.M. (1989). Computerized smoking cessation program for the worksite: Treatment outcome and feasibility. Journal of Consulting and Clinical Psychology, 57, 619-622. 
Campbell, M.K., De Vellis, B.M., Strecher, V.J., Ammerman, A.S., De Vellis, R.F., \& Sandler, R.S. (1994). Improving dietary behavior: The effectivesness of tailored messages in primary care settings. American Joumal of public Health, 84, 783-787.

Chapman, S., Wong, W.L., \& Smith, W. (1993). Self-exempting beliefs about smoking and health: Differences between smokers and ex-smokers. American Joumal of Public Health, 83, 215-219.

Christen, A.G., Christen, J.A. (1994). Why is cigarette smoking so addicting? An overview of smoking as a chemical and process addiction. Health Values, $18,17-24$.

Coelho, R.J. (1984). Self-efficacy and cessation of smoking. Psychological Reports, 54, 309-310.

Cohen, S., Lichtenstein, E., Prochaska, J.O., Rossi, J.S., Gritz, E.R., Carr, C.R., Orleans, C.T., Schoenbach, V.J., Biener, L., Abrams, D., DiClemente, C., Curry, S., Marlatt, G.A., Cummings, K.M., Emont, S.L., Giovino, G., \& Ossip-Klein, D. (1989). Debunking myths about self-quitting. Evidence from 10 prospective studies of persons who attempt to quit smoking by themselves. American Psychologist, 44, 1355-1365.

Condiotte M.M., \& Lichtenstein, E. (1981). Self-efficacy and relapse in smoking cessation programs. Joumal of Consulting and Clinical Psychology, 49, 648658.

Crittenden, K.S., Manfredi, C., Lacey, L., Warnecke, R., \& Parsons, J. (1994). Measuring readiness and motivation to quit smoking among women in public health clinics. Addictive Behoviors, 19, 497-507.

Cummings, K.M., Emont, S.L., Jaèn, C., \& Sciandra. R. (1988) Format and quitting instructions as factors influencing the impact of a self-administered quit smoking program. Health Education Quarterly, 15, 199-216.

Curry, S.J., McBride, C., Grothaus, L.C., Louie, D., \& Wagner, E.H. (1995). A randomized trial of self-help materials, personalized feedback, and telephone counseling with nonvolunteer smokers. Journal of Consulting and Clinical Psychology, 63, 1005-1014.

Curry, S.J., Wagner, E.H., \& Grothaus, L.C. (1991). Evaluation of intrinsic and extrinsic motivation interventions with a self-help smoking cessation program. Joumal of Consulting and Clinical Psychology, 59, 318-324.

Davis, A.L., Faust, R., Ordentlich, M. (1984). Self-help smoking cessation and maintenance programs: A comparative study with 12-month follow-up by the American Lung Association. American Journal of Public Health, 74, 12121217.

De Vries, H. (1989). Prevention of cancer: Towards a national ABC Framework. European Journal of Cancer and Clinical Oncology, 25, 1025-1033.

De Vries, H., \& Backbier, E. (1994). Self-efficacy as an important determinant of quitting among pregnant woman who smoke: the $\varnothing$-pattern. Preventive Medicine, 23, 167-174. 
De Vries, H. Backbier, E., Dijkstra, M., Van Breukelen, G., Parcel, G., \& Kok, G. (1994). A Dutch social influence smoking prevention approach for vocational school students. Health Education Research, 9. 365-374.

De Vries, H., Backbier, E., Kok, G.J., \& Dijkstra, M. (1995). The impaci of social influences in the context of attitude, self-efficacy, intention and previous behavior as predictors of smoking onset. Joumal of Applied Social Psychology, 25, 237-257.

De Vries, H., Dijkstra, M., \& Kuhlman, P. (1988). Self-efficacy: The third factor besides attitude and subjective norm as a predictor of behavior intention. Healih Education Research, 3, 273-282.

De Vries, H., \& Kok, G. (1996). Gezondheidsbevordering: Een toepassing wan het ABC-planningsmodel [Health education: $A$ application of the $A B C$ planning modell. Gedrag \& Gezondheid, 24, 6, 342-352.

De Vries, $H_{\text {. }}$ \& Mudde, A.N. (in press). Predicting stage transitions for smoking cessation applying the Attitude - Social influence - Efficacy model. Psychology and Health.

De Vries, H., Willemsen, M., Brug, J., Dijkstra, A., \& Berben, J. (1995). Voorlichting op maat; een nieuwe interactieve voorlichtingsmethode [tailored education; a new interactive method of health education]. Tijdschrift Gezondheidsbevordering, 16, 59-70.

DiClemente, C.C. (1981). Self-efficacy and smoking cessation Maintenance: A Preliminary report. Cognitive Therapy and Research, 5, 175-187.

DiClemente, C.C., Prochaska, J.O., Fairhurst, S.K., Velicer, W.F., Velasquez, M.M. \& Rossi, J.S. (1991). The process of smoking cessation: An analysis of precontemplation, contemplation and preparation stages of change. Journal of Consulting and Clinical Psychology, 59, 295-304.

DiClemente, C.C., Prochaska, J.O., \& Gibertini, M. (1985). Self-efficacy and the stages of self-change of smoking. Cognitive therapy and Research, 9, 181200.

Dijkstra, A. (1996). Dilemma's in de gezondheidsvoorlichting [Dilemmas in health education]. Psychologie en Maatschappij, 20, 231-243.

Dijkstra, A., Bakker, M., \& De Vries, H. (1997). Subtypes within a precontemplating sample of smokers: A preliminary extension of the stages of change. Addictive Behaviors, 22, 327-337.

Dijkstra, A., De Vries, H., \& Bakker, M. (1995). Voor- en nadelen, eigen effectiviteit en fasen van verandering bij stoppen met roken [Pros an cons, self-efficacy and the stages of change in smoking cessation]. Gedrag \& Gezondheid, 23, 217-228.

Dijkstra, A., De Vries, H., \& Bakker, M. (1996). The pros and cons of quitting, self-efficacy and the stages of change in smoking cessation. Journal of Comsulting and Clinical Psychology, 64, 758-763.

Dijkstra, A., De Vries, H., Roijackers, J. (1996). Efficacy of attitudinal versus self-efficacy enhancing information in motivating smokers in different motivational stages to quit: A field experimental trial. Paper presented at the 10th European Health Psychology Society Conference, Dublin. 
Dijkstra, A., De Vries, H., \& Roijackers, I. (in press-a). Computerized tailored feed-back to change cognitive determinants of smoking: A Dutch field experiment. Healh Education Research.

Dijkstra, A., De Vries, H., \& Roijackers, J. (in press-b). Long-term effectiveness of computer-generated tailored feed-back in smoking cessation. Healih Education Research.

Dijkstra, A., De Vries, H., \& Roijackers, J., Breukelen, van, G. (1997). Validering van een veranderingsfasenmodel bij stoppen met roken: Een veldexperiment [The validation of a stage model in smoking cessation: $A$ field experiment]. ASPO-bundel toegepaste sociale psychologie, 11, 172-185.

Dijkstra, A., De Vries, H., \& Roijackers, J., Breukellen, van, G. (in press-d). Tailored interventions to communicate stage-matched information to smokers in different motivational stages. Joumal of Consulting and Clinical Psychology.

Dijkstra, A., De Vries, H., \& Roijackers, J., Breukelen, van, G. (in press-e). Computerized taillored feed-back to stimulate precontemplators to quit smoking: Three basic efficacy questions. Health Psychology.

Dijkstra, A., De Vries, H., Roijackers, J. \& Mudde, A. (1997). Psychomsocial determinants of stage transition. Paper presented at the 11 th European Health Psychology Society Conference, Bordeaux.

Dijkstra, A., Roijackers, J., \& De Vries, H. (in press-c). Smokers in four stages of readiness to change. Addictive Behaviors.

Doll, R., Peto, R., Wheatly, K., Gray, R, \& Sutherland, I. (1994). Mortality in relation to smoking: 40 years' observations on male British doctors. Britisch Medical Journal, 309, 901-911.

Dutch Foundation on Smoking and Health (1992). Annual report. The Hague, The Netherlands: Author:

Dutch Foundation on Smoking and Health (1995). Annual report. The Hague, The Netherlands: Author.

Eagly, A.H., \& Chaiken, S. (1993). The Psychology of Attitudes. Orlando, FL: Harcourt Brace Jovanovich.

Ershoff, D.H., Mullen, P.D., \& Quinn, V.P. (1989). A randomized trial of serialized self-help smoking cessation program for pregnant women in an HMO. American Jounal of Public Health $79,182-187$.

Etter, J.F., Perneger, T.V., \& Ronchi, A. (1997). Distribution of smokers by stage: International comparison and association with smoking prevalence. Preventive Medicine, 26. 580-585.

Fava, J.L., Velicer, W.I., \& Prochaska, J.O. (1995). Applying the transtheoretical model to a representative sample of smokers. Addictive Behaviors, 20, 189-203.

Festinger, L. (1957). A theory of cognitive dissonance. London: Tavistock Press. Gebhardt, A.G. (1997). Health Behaviour Goal Model: Towards a theoretical framewark for health behaviour change. Doctoral dissertation, University of Leiden, The Netherlands. 
Gibbons, F.X. (1990). Self-evaluation and self-perception: The role of attention in the experience of anxiety. Anxiety Research, 2, 153-163.

Ginnis, J.M. \& Foege, W.H. (1993). Actual causes of death in the United States. Journal of the American Medical Association, 270, 2207-2212.

Glynn T.J., Boyd, G.M., \& Gruman, J.C. (1990) Essential elements of selfhelp/minimal intervention strategies for smoking cessation. Health Education Quarterly, 17, 329-345.

Godin, G., \& Kok, G. (1996). The theory of planned behavior: A review of applications to health-related behaviors. American Joumal of Healh Promotion, 11, 87-98.

Godin, G., Valois, P., Lepage, L., \& Desharnais, R. (1992). Predictors of smoking behavior: An application of Ajzen's theory of planned behavior. British Journal of Addiction, 87, 1335-1343.

Heatherton, T.F., Kozlowski, L.T., Frecker, R.C., \& Fagerström, K.O. (1991). The Fagerström test for nicotine dependence: a revision of the Fagerström tolerance questionnaire. British Journal of Addiction, 86, 1119-1127.

Heyting, A., Tolboom, J.T.B.M., \& Essers, J.G.A. (1992). Statistical handling of drop-outs in longitudinal clinical trials. Statistics in Medicine, 11, 20432061.

Janis, I.L., \& Mann, L. (1977). Decision making: A psychological analysis of conflict, choice, and commitment. New York: Collier Macmillan.

Janz, N.K., \& Becker, M.H. (1984). The Health Belief Model: A decade later. Health Education Quarterly, 11, 1-47.

Jarvis, M.J. (1994). A profile of tobacco smoking. Addiction, 89, 1371-1376. Jones, E.E., \& Sigal, H. (1971). The bogus pipeline: A new paradigm for measuring effect and attitude. Psychological Bulletin, 76, 349-364.

Kavanagh, D.J., Pierce, J., Sing, K.L., \& Shelley, J. (1992). Self-efficacy and social support as predictors of smoking after a quit attiempt. Psychology and Health, 8, 243-255.

Kazdin, A.E. (1986). The evaluation of Psychotherapy: Research designs and methodology. In S.L. Garfield \& A.E. Bergin (Eds.), Handbook of Psychotherapy and Behavior Change. New York: John Wiley \& Sons.

Keltner, D., \& B $B$ uswell, B.N. (1996). Evidence for the distinctness of embarrassment, shame, and guilt: A study of recalled antecedents and facial expressions of emotion. Cognition \& Emotion, 10, 155-171.

Kok, G.J., Schaalma, H., De Vries, H., Parcel, G., \& Paulussen, T. (1996). Social Psychology and Health Education. In W. Stroebe \& M. Hewstone (Eds.), European Review of Social Psychologv, Volume 7 (p. 241-282). John Willey \& Sons Lid.

Kreuter, M.W., Strecher, V.J. (1996). Do tailored behavior change messages enhance the effectiveness of health risk appraisal? Results from a randomized trial. Health Education Research, 11, 97-105.

Kristeller, J.L., Rossi, J.S., Ockene, J.K., Goldberg, R., \& Prochaska, J.O. (Processes of change in smoking cessation: A cross-validation study in cardiac patients. Jownal of Substance Abuse, 4, 263-276. 
Lavy, E. (1993). Attenional bias and anxiety: Conceptual issues and empirical data. Doctoral dissertation. Maastricht University.

Lechner, L., Brug, J., \& De Vries, H. (in press). Misconceptions of fruit and vegetable consumption: Differences between objective and subjective estimation of intake. Joumal of Nutrition Education.

Lechner, L., Brug, J., De Vries, H., Van Assema, P., \& Mudde, A. (in press). Stages of change for fruit, vegetable and fat intake: consequences of misconception. Health Education Research.

Lee, C. (1989). Perceptions of immunity to disease in adult smokers. Jounal of Behavioral Medicine, 12, 267-276.

Lichtenstein, E., \& Glasgow, R.E. (1992). Smoking cessation: What have we learned over the past decade? Journal of Consulting and Clinical Psychology, $60,518-527$.

Maddux, J.E., \& Rogers, R.W. (1983). Protection motivation and self-efficacy: A revised theory of fear appeals and attitude change. Journal of Experimental Social Psychology, 19, 469-479.

Manstead, A.S.R., \& Parker, D. (1995). Evaluating and extending the theory of planned behavior. European Review of Social Psychology, 6, 69-95.

Maibach, E., \& Parrot, R.L. (1995). Designing health messages, approaches from communication theory and public health practice. Thousand Oaks: Sage.

Marlatt, G.A., \& Gordon, J.R. (1985). Relapse prevention: Maintenance strategies in the treatment of addictive behaviors. New York, London: Guilford Press.

McConnaughy, E.A., DiClemente, C.C., Prochaska, J.O., \& Velicer, W.F. (1989). Stages of change in psychotherapy: A follow-up report. Psychotherapy, 26, 494-503.

McConnaughy, E.A., Prochaska, J.O., \& Velicer, W.F. (1983). Stages of change in psychotherapy: Measurement and sample profiles. Psychotherapy. Theory, Research and Practice, 20, 368-375.

McFall, S.L., Michener, A., Rubin, D., Flay, B.R., Mermelstein, R.J., Burton, D., Jelen, P., \& Warnecke, R.B. (1993). The effects and use of maintenance newsletters in a smoking cessation intervention. Addictive Behaviors. 18. $151-158$.

Mclntyre, K.O., Lichtenstein, E., \& Mermelstein, R.J. (1983). Self-efficacy and relapse in smoking cessation: A replication and extension. Joumal of Consuling and Clinical Psychology, 51, 632-633.

McMaster, C, \& Lee, C. (1991). Cognitive dissonance in tobacco smokers. Addictive Behaviors, 16, 349-353.

Miller, W.R., \& Rollnick, S. (1991). Morwational interviewing. New York: The Guilford Press.

Mudde, A.N., De Vries, H., Willemsen, M., \& Assema, P. van (1994) In R. Richmond (Eds), Interventions for smokers: An international perspective ( $\mathrm{p}$. 293-322). New York: Williams \& Wilkins. 
Mudde, A.N., Dolders, M., \& De Vries, (1994). Publieksevaluatie van de actie: Volwassen bevolking [Evaluation of the action: The adult population]. In B. Baan, M.H.M. Breteler, \& G.A.J. Van Der Rijt (Eds.), Samen stoppen met roken [Quitting smoking together] (p. 22-44). The Hague, The Netherlands: Stichting Volksgezondheid en Roken.

Mudde, A.N., Kok, G.J., \& Strecher, V.J. (1995). Self-efficacy as a predictor for the cessation of smoking: Methodological issues and implications for smoking cessation programs. Psychology and Health, 10, 353-367.

O'Connel, K.A., \& Martin, E.J. (1987) Highly tempting situations associated with abstinence, temporary lapse, and relapse among participants in smoking cessation programs, Joumal of Consulting and Clinical Psychology, 55, 367371 .

Orleans, C.T. (1993). Treating nicotine dependence in medical setting: a stepped-care model. In C.T. Orleans \& J. Slade (Eds.), Nicotine addiction, principles and management (p. 145-161). New York: Oxford University Press.

Owen, N., Ewins, A.L., \& Lee, C. (1989). Smoking cessation by mail: A comparison of standard and personalized correspondence course formats. Addictive Behowiors, 14, 355-363.

Pallonen, U.E., Fava, J.L., Salonen, T.S., \& Prochaska, J.O. (1992). Readiness for smoking change among middle-aged finnish men. Addicitve Behaviors, 17, 415-423.

Pallonen, U.E., Leskinen, L., Prochaska, J.O., Willey, C.J., Kääriäinen, R., \& Salonen, J.T. (1994). A 2-year self-help smoking cessation manual intervention among middle-aged Finnish men: An application of the Transtheoretical model. Preventive Medicine, 23, 507-514.

Pervin, L.A. (1989). Personality: Theory and Research. Singapore: John Wiley \& Sons, Inc.

Peto, R., Lopez, A.D., Boreham, J., Thun, M., \& Heath, C. (1994). Mortaility from smoking in developed countries: 1950 - 2000: indirect estimates from national vital statistics. New York: Oxford University Press.

Petraitis, I., Flay, B.R., \& Miller, T.Q. (1995). Reviewing theories of adolescent substance use: Organizing pieces in the puzzle. Psychological Bulletin, 1,6786.

Petty, E.P., \& Cacioppo, J.T. (1986). Communication and Persuasion, Central and Peripheral Routes to Atritude Change. New York: Springer-Verlag.

Pomerleau, C.S., Carton, S.M., Lutzke, M.L., Flessland, K.A., \& Pomerleau, O.F. (1994). Reliability of the Fagerström questionnaire and the Fagerström test for nicotine dependence. Addictive Behaviors, 19. 33-39.

Powers, W.T. (1973). Feedback: Beyond Behaviorism. Science, 179, 351-356. Prochaska, J.O. (1.984). Systems of psycho:herapy: A transtheoretical analysis. Homewood ,Il: Dorsey Press.

Prochaska, J.O. (1991). Prescribing to the stage and level of phobic patients. Psychotherapy, 28, 463-468. 
Prochaska, J.O. (1994). Strong and weak principles for progressing from precontemplation to action on the base of twelve problem behaviors. Health Psychology, 13, 1-5.

Prochaska, J.O., \& DiClemente, C.C. (1983). Stages and processes of seltchange of smoking: Toward an integrative model of change. Joumal of Consulting and Clinical Psychology, 51, 390-395.

Prochaska, J.O., \& DiClemente, C.C. (1984). Self-change processes, self-efficacy and decisional balance across five stages of smoking cessation. Advances in Cancer Control: Epidemiology and Research, 131-140.

Prochaska, J.O., DiClemente, C.C., \& Norcross, J.C. (1992). In search of how people change, applications to addictive behaviors. American Psychologist, 47, 1102-1114.

Prochaska, J.O., DiClemente, C.C., Velicer, W.F., Gimpil S., \& Norcross, J.C. (1985). Predicting change in smoking status for self-changers. Addictive Behaviors, 10, 395-406.

Prochaska, J.O., DiClemente, C.C., Velicer, W.F., \& Rossi, J.S. (1993). Standardized, individualized, interactive, and personalized self-help programs for smoking cessation. Health Psychology, 12, 399-405.

Prochaska, J.O., Norcross, J.C., Fowler, J.L., Follick, M.J., \& Abrams, D.B. (1992). Attendance and outcome in a work site weight control program" processes and stages of change as process and predictor variables. Addictive Behaviors, 17, 35-45.

Prochaska, J.O., Redding, C.A., Harlow, L.L., Rossi, J.S., \& Velicer, W.F. (1994). The transtheoretical model of change and HIV prevention: A review. Health Education Quarterly, 21, 471-486.

Prochaska, J.O., Velicer, W.F., DiClemente, C.C., \& Fava, J. (1988). Measuring processes of change: Applications to the cessation of smoking. Joumal of Consulting and Clinical Psychology, 56, 520-528.

Prochaska, J.O., Velicer, W.F., Guadagnoli, E., Rossi, J.S., \& DiClemente, C.C. (1991). Patterns of change: Dynamic typology applied to smoking cessation. Multivariate Behavioral Research, 26, 83-107.

Prochaska, J.O., Velicer, W.F., Rossi, J.S., Goldstein, M.G., Marcus, B.H., Rakowski, W., Fiore, C. Harlow, L.L., Redding, C.A., Rosenblorm, D., \& Rossi, S.R. (1994). Stages of change and decisional balance for 12 problem behaviors. Heath Psychology. 13, 39-46.

Prussia, G.E., \& Kinicki, A.J. (1996). A motivational investigation of group effectiveness using social-cognitive theory. Journal of Applied Psychology, 81, 187-198.

Richard, R., \& Van der Pligt, J., \& De Vries, N. (1995). Anticipated affective reactions and prevention of AIDS. British Journal of Social Psychology, 34. $9-21$.

Rimer, B.K., Orleans, C.T., Fleisher, L., Cristinzio, S., Resch, N., Telepchak, J., \& Keintz, M. (1994). Does tailoring matter? The impact of a tailored quide on ratings and short-term smoking-related outcomes for older smokers. Health Education Research, 9, 69-84. 
Robinson, L.D. \& Jewell, N.P. (1991). Some surprising results about covariate adjustement in logistic regression. International Statistical Review, 58, 227240.

Rollnick, S., Heather, N., Gold, R., \& Hall, W. (1992). Development of a short 'readiness to change' questionnaire for use in brief, oppotunistic interventions among excessive drinkers. British Journal of Addiction, 87, 743-754.

Rosenstock, 1. (1974). The health belief model and preventive health behavior. Health Education Monographs, 2, 354-386.

Sacks, J.ll, \& Nelson, D.E. (1994). Smoking and injuries: An overview. Preventive Medicine, 23, 515-520.

Schwarzer, R. (1992). Self-efficacy in the adoption and maintenance of health behaviors: Theoretical approaches and a new model. In R. Schwarzer (Eds.) Self-efficacy: Thought control of action (p. 217-244). Washington: Hemisphere Publishing Coorporation.

Schwartz, J.L. (1987). Smoking cessation methods: The United States and Canada, 1978-1985. Bethesda, Maryland: Department of Health and Human Services.

Shiffman, S, Paty, J.A., Gny/s, M., Kassel, J.A., \& Hickcox, M. (1996) First lapses to smoking: Within-subject analysis of real-time reports, Journal of Consulting and Clinical Psychology, 64, 366-379.

Skinner, C.S., Siegfried, J.C., Kegler, M.C., \& Strecher, V.J. (1993). The potential of computers in patient education. Patient Education and Counseling, 22, 27-34.

Skinner, C.S., Strecher, V.J., \& Hospers, H. (1994). Physicians' recommendations for mammography: Do tailored messages make a difference? American Journal of Public Health, 84, 43-49.

Skinner, E.A. (1996). A guide to constructs of control. Journal of Personality and Sociai Psychology, 71. 549-570.

Slama, K. (1995). Tobacco \& Health. New York: Plenum Press.

Snow, M.G., Prochaska, J.O., \& Rossi, J.S. (1992). Stages of change for smoking cessation among former problem drinkers: A cross-sectional analysis. Journal of Substance Abuse, 4, 107-116.

Solomon, L.J., \& Flynn, B.S. (1993). Women who smoke. In C.T. Orleans, \& J. Slade (Eds.), Nicotine Addiction: principles and management (p. 339-349). New York: Oxford University Press.

Sorensen, G., \& Pechacek, T.F. (1987). Attitudes toward smoking cessation among men and women. Journal of Behovioral Medicine, 10, 129-137.

Strecher, V.J., Kreuter, M., Den Boer, D.J., Kobrin, S., Hospers, H.J., \& Skinner, C.S. (1994). The effect of computer-tailored smoking cessation messages in familly practice settings. The Joumal of Family Practice, 39, $262-268$.

Strecher, V.J., McEvoy DeVellis, B., Becker, M.H., \& Rosenstock, I.M. (1986). The role of self-efficacy in achieving health behavior change. Health Education Quarterly, 13, 73-91. 
Strecher, V.J., Rimer, B.K., \& Monaco, K.D. (1989). Development of a new seif-help guide-Freedom from smoking for you and your family. Heolh Education Quarterly, 16, 101-112.

Stroebe, W. \& Stroebe, M.S. (1995). Social Psychology and Health. Buckingham: Open University Press.

Sutton, S. (1996). Can 'stages of change' provide guidance in the treatment of addictions? A critical examination of Prochaska \& DiClemente's model. In G. Edwards \& C. Dare (Eds.), Psychotherapy, Psychological Treatments and the Addictions, (pp. 189-205). Cambridge: Cambridge University Press.

Swan, G.E., Ward, M.M., \& Jack, L.M. (1996) Abstinence effects as predictors of 28-day relapse in smokers, Addictive Behaviors, 21, 481-490.

Velicer, W.F., DiClemente, C.C., Prochaska, J.O., \& Brandenburg, N. (1985). Decisional balance measure for assessing and predicting smoking status. Journal of Personality and Social Psychology, 48, 1279-1289.

Velicer, W.F., DiClemente, C.C., Rossi, J.S., \& Prochaska, J.O. (1990). Relapse situations and self-efficacy: An integrative model. Addictive Behaviors, 15, 271-283.

Velicer, W.F., Fava, J.L., Prochaska, J.O., Abrams, D.B., Emmons, K.M., \& Pierce, J.P. (1995). Distribution of smokers by stage in three representative samples. Preventive Medicine, 24, 401-411.

Velicer, W.F., Hughes, S.L., Fava, J.L., Prochaska, J.O., \& DiClemente (1995). An empirical typology of subjects within stage of change. Addictive Behaviors, 20, 299-320.

Velicer, W.F., Prochaska, J.O., Rossi, J.S., \& Snow, M.G. (1992). Assessing outcome in smoking cessation studies. Psychological Bulletin, $/ I I, 23-41$.

Velicer, W.F., Prochaska, J.O., Bellis, J.B., DiClemente, C.C., Rossi, J.S., Fava, J.L., Steiger, J.H. (1993). An expert system intervention for smoking cessation. Addictive Behaviors, 18, 269-290.

Velicer, W.F., Rossi, J,S., Prochaska, J.O., \& DiClemente, C.C. (1996). A criterion measurement model for health behavior change. Addictive Behaviors, 21, 555-584.

Velicer, W.F., Rossi, J.S., Ruggiero, L., \& Prochaska, J.O. (1994). Minimal intervention appropriate for an entire population of smokers. In: R. Richmond (Eds.), Interventions for smokers: An international perspective ( $1.69-92)$. New York: Williams \& Wilkins.

Weinstein, N.D. (1988). The precaution Adoption Process. Health Psychology, 7, 355-386.

Willemsen, M.C., De Vries, H., Van Breukelen, G., \& Oldenburg, B. (1996), Determinants of intention to quit smoking among Dutch employees: The influence of the social environment. Preventive Medicine, 25, 195-202. 


\section{Summary}

Despite the negative health consequences of smoking tobacco, many smokers are not motivated to quit smoking: the percentage of smokers who are not planning to quit within the next six months is about $70 \%$ in the Netherlands and about $40 \%$ in the U.S.A. The present thesis is about those smokers with low motivation to quit smoking and the development and effectiveness of computer-generated tailored interventions to increase their motivation to quit. Smoking cessation is not viewed as a dichotomy but as a process from no motivation to change, to the eventual internalization of the new (non-smoking) behavior. In this thesis, this process is described in terms of the stages of change.

In order to be able to develop an intervention aimed at encouraging these low motivated smokers to quit, it is essential to know what the psycho-social determinants of smoking and smoking cessation are, since interventions will have to change these determinants. Therefore, in the first part of this thesis, three studies are presented which give insight into the psycho-social determinants of smoking and smoking cessation.

In Chapter 2 a cross-sectional analysis of smokers and ex-smokers in five stages of readiness to change is presented. Smokers in precontemplation, contemplation, preparation, action and maintenance were compared on expected positive and negative outcomes (pros and cons of quitting) and selfefficacy expectations. The results show that, first, the pattern of the pros and cons of quitting through the stages was almost identical to the pattern found with the pros and cons of smoking (Decisional Ballance) assessed within the framework of the transtheoretical model (TTM). That is, the "strong and weak principle of behavior change", as defined within the TTM, was replicated: the maximum difference within the first four stages (precontemplation, contemplation, preparation and action) on the pros of quitting, was 1.2 standard deviation and the maximum difference within these stages on the cons of quitting was 0.5 standard deviation. These findings were considered to be a validation of the operationalizations of the psychological constructs used. Second, as hypothesized on the basis of the so-called $\varnothing$-pattern, the pros of quitting discriminated better between the early stages (precontemplation, contemplation and preparation), whereas self-efficacy expectations discriminated better between the later stages (action and maintemance). This suggests that this differential pattern of the pros of quitting and self-efficacy through the stages may have consequences for stage-matched interventions: precontemplators may need outcome information to move forward through the stages, contemplators may need outcome and selfefficacy enhancing information and preparers may need self-efficacy enhancing information only.

In Chapter 3, a stage model which classifies smokers in four instead of in three early stages is investigated. In the group of smokers who were not planning to quit within the next six months, two groups of smokers with 
differing readiness to change were distinguished: immotives, who were not planning to quit within the next five years or were planning to never quit and precontemplators, who were planning to quit within the next five years but not yet within the next six months. Thus, the following four groups of smokers with increasing readiness to change are distinguished: immotives, precontemplators, contemplators and preparers. The pattern of the pros and cons of quitting and self-efficacy through these stages and the predictive validity of the stage model were investigated.

To obtain a more differentiated view of the cognitive differences between the four stages, they were compared on the scores on the factors within the pros and cons of quitting and self-efficacy. A principal component analysis showed the expected factor structure within the items measuring the pros of quitting and self-efficacy. Within the pros of quitting, two factors referring to physical outcomes were found, one referring to social outcomes and one referring to self-evaluative outcomes. One item assessing the monetary outcomes of quitting did not fit into any other factor. The factors differed in the amount to which they discriminated between the four stages. Within the items assessing self-efficacy, two factors were found: self-efficacy with regard to coping with the urge to smoke in social situations and self-efficacy with regard to coping with the urge to smoke in emotional situations.

The results of the comparisons of the four stages on these cognitive factors showed that smokers in the four stages differed on all factors. However, the higher the readiness to quit, the smaller the number of factors which discriminated between two consecutive stages: immotives and precontemplators differed on all factors, whereas contemplators and preparers differed on only one factor (i.e. self-evaluative outcomes). The results with regard to the predictive validity showed that the stages measured at Tl, were indeed predictive of quitting (after three and after fourteen months). Being an immotive or a precontemplator was predictive of quitting measured with three behavioral measures at both follow-ups. Being a precontemplators or a contemplator was predictive of quitting on two measures after three months and on one measure after fourteen months. Finally, being a contemplator or a preparer was predictive of quitting on all three measures after three months and on two measures after fourteen months. It is concluded that the four stages of readiness to change are strong predictors of quitting behavior, but that the distinction between precontemplation and contemplation is more strongly supported by the differences in psycho-social determinants.

In Chapter 4 , the influences of expected outcomes on intention to quit and behavior are further mapped out. The Self-Evaluation-Motivation model depicts physical, social and self-evaluative outcome expectations, two selfevaluation inhibiting processes (i.e. dissonance-reducing cognitions and attentional change processes), personal standards and their influence on intention to quit and quitting behavior. The results of multivariate regression analyses revealed the following relations.

First, the influence of physical and social outcomes and of dissonance 
reducing cognitions and attentional change processes on intention and behavior, was mediated by self-evaluation. Second, perceived long-term health outcomes were not related to self-evaluation when smokers used few attentional change processes. Third, the influence of dissonance-reducing cognitions and attentional change processes on self-evaluation was partly mediated by their influence on physical and social outcome expectations. Suggestions for interwentions to increase motivation to quit smoking are given.

It is concluded that self-evaluation is a central psycho-social determinant of intention and behavior and that, hence, interventions should increase selfevaluation. Self-evaluation might be increased by: 1) increasing particularly the perception of the short-term health consequences and the social consequences of quitting; 2) restructuring the dissonance-reducing cognitions; 3) increasing the use of attentional change processes; 4) explicitly addressing the discrepancy between a persons personal standard and the consequences of smoking behavior.

In Part I of this thesis the psycho-social determinants of smoking and smoking cessation are examined. In Part II, the knowledge about the determinants is used to develop a tailored intervention. Subsequently, the effectiveness of the computer-tailored interventions is tested in three large randomized field trials.

In Chapter 5, three phases in the development of a tailored intervention are outlined. In the first phase, the intervention objectives are formulated on the basis of analyses of the cognitive determinants. The objectives define the desired psychological and behavioral state to be brought about by the specific part of the tailored letter. Furthermore, the structure of the letter is designed. In the second phase, a tailoring matrix is developed for each message objective. The matrix depicts the relevant characteristics on which the message adapted. For each cell in the tailoring matrix, the messages are written and the two clusters of tailoring working mechanisms, adaptation of the information and feedback, are included. To be able to produce interventions which take into account and/or feed back personal characteristics, an individual assessment has to be made. Therefore, a tailoring questionnaire is developed. The tailoring matrix and the tailoring questionnaire are combined to form the functional design, which is the basis for the tailoring computer program. In the third phase, it must be ensured that the resulting tailored intervention, composed of different messages which may be adapted to different individual characteristics, is one coherent intervention. Lastly, an attractive and readable lay-out is designed.

In Chapter 6, taillored interventions are tested in a large randomized field trial $(N=1540)$ among immotives, precontemplators, contemplators and preparers. A special case of tailored interventions is used: individualized stage-matched interventions. Stage-matched interventions are tailored interventions, in that they take into account the readiness to change of an individual. The currently used individualized stage-matched interventions offer 
stage-matched information tailored to several individual characteristics, such as level of expected outcomes, situational self-efficacy, number of cigarettes and gender.

Smokers in the four stages were randomly assigned to a condition offering: 1) outcome information; 2) self-efficacy enhancing information; ) both sorts of information or; 4) no information. The data on 1540 smokers were analyzed, stratified by stage. The primary outcome measure was stage transition. On the basis of the hypotheses generated in the study in Chapter 2, it was expected that immotives would benefit most from outcome information only, that precontemplators and contemplators would benefit most from a combination of self-efficacy enhancing information (i.e. information on skills) and outcome information, and that preparers would benefit most from selfefficacy enhancing information only.

At the three months follow-up, the hypotheses with regard to stagematched information for immotives and precontemplators were partly verified: Compared to the no-information control group, immotives benefitted from an intervention which contained outcome information (in combination with self-efficacy enhancing information or not) and precontemplators benefitted from an intervention containing self-efficacy enhancing information (in combination with outcome information or not). However, the more stringent test comparing smokers who were assigned to a matched condition to smokers who were assigned to a mismatched condition revealed no significant differences. With regard to contemplators and preparers, the following was found: compared to the no-information control group, contemplators benefitted most from the condition containing both sorts of information, whereas preparers benefitted most from the condition containing selfefficacy enhancing information only. The more stringent match-mismatch test in contemplators and preparers supported these findings.

In Chapter 7, the long-term effectiveness of tailored interventions in a sample of smokers with varying readiness to quit is assessed using the smoker cohort from the study in Chapter 6 . The results from the fourteen months follow-up can be summarized as follows. Compared to the no-information condition, all three experimental conditions led to significantly more smokers who had engaged in a 24-hour quit attempt. However, no experimental condition led to more seven-days quitting than the no-information condition. With regard to continuous abstinence, the experimental condition offering a combination of outcome information and self-efficacy enhancing information had a significant effect, compared to the no-information condition. It is concluded that a minimal 6-page tailored intervention can be beneficial in supporting smokers to quit smoking, even after fourteen months.

In Chapter 8, four tailored interventions and one non-tailored look-alike intervention are tested in a large randomized field trial $(N=752)$ among immotives and precontemplators. An additional tailoring system was developed, producing the so-called multiple tailored interventions. A multiple tailored intervention consists of three consecutive individualized tailored 
letters which are mailed to the individual at intervals. In the present experiment the interval was one month. The multiple tailored intervention contained roughly the same information as the single tailored intervention, but in the former the information was distributed over the three letters, each comprising four or five pages of information. The content of the first letter was on the basis of the mailed questionnaire, whereas the following two letters were on the basis of two 10-minute telephone questionnaires. Besides this intervention, a single tailored intervention and a multiple tailored intervention referring to an additional self-help guide were developed. Furthermore, a look-alike but non-tailored intervention was printed on the same paper as the tailored letters and with the same lay-out.

Smokers with explicit low intention to quit were randomly assigned to one of five conditions: 1) the multiple tailored intervention with an additional self-help guide; 2) the multiple tailored intervention only; 3) a single tailored intervention with an additional self-help guide; 4) a single tailored intervention only; or 5) a non-tallored look-alike intervention. The follow-up assessment was four months after the intervention.

The results showed that the single tailored intervention without the additional self-help guide had no surplus value compared to a non-tailored look-alike intervention. The addition of a self-help guide to a tailored intervention was only useful in highly dependent smokers and the multiple tailored intervention was clearly more effective than the single tailored intervention.

In Chapter 9, three self-help interwentions are tested in a large randomized field trial $(\mathrm{N}=843)$ among immotives and precontemplators. Two different tailored smoking cessation self-help interventions (the multiple and single tailored intervention) and one standardized 46-page smoking cessation selfhelp guide are compared to a no-information control group and to each other.

The results showed that, in immotives, only the tailored interventions were more effective than no information. Furthermore, the multiple tailored intervention was more effective than both other self-help interventions, in immotives, but the single tailored intervention was not more effective than the standardized self-help guide. A similar pattern was found with regard to the cognitive changes the interventions brought about: the multiple tailored intervention led to the largest increases in the pros of quitting, self-efficacy and two attentional change processes: Consciousness Raising and Environmental Reevaluation. The interventions did not differentially influence the cons of quitting and the attentional change process of Social. Liberation. In precontemplators, surprisingly, the self-help materials had no effect. This appeared to be due to the high percentage of stage transition in the control group.

In Chapter 10, the findings of the previous eight studies presented are integrated. First, the findings are discussed with regard to outcome expectation (physical, social and self-evaluative), with regard to self-efficacy expectation (to cope with emotions and to cope with social situations) and with 
regard to the self-evaluation inhibiting factors (dissonance-reducing cognitions and attentional change processes). Second, the effectiveness of the tailored interventions is addressed and the discussion of the results is structured according to five evaluation strategies. Third, some methodological issues are addressed. Fourth, the application of the stage model and the tailored interventions are discussed. A typology of smokers in the four stages of readiness to change is presented. Fifth, some recommendations are given with regard to the future investigations of cognitive determinants, the stages of change and the development of tailored interventions. 


\section{Samenvatting}

Ondanks de negatieve gevolgen van roken voor de gezondheid, is $70 \%$ van de Nederlandse rokers niet van plan om in de komende zes maanden een stoppoging te doen. In de Verenigde Staten is dit percentage zo'n $40 \%$. Deze rokers worden vooroverwegers genoemd. Het onderhavige proefschrift gaat over de ontwikkeling en de effectiviteit van computer-gegenereerde geïndividualiseerde interventies voor deze groep rokers. Deze interventies zijn ontwikkeld om hen aan te moedigen om te stoppen met roken. Stoppen met roken wordt niet gezien als een dichotomie (wel/niet stoppen), maar als eem proces van geen motivatie om te veranderen tot het internaliseren van het nieuwe (niet-roken) gedrag. Dit proces zal beschreven worden in termen var veranderingsfasen.

Om een stoppen-met-roken interventie te kunnen ontwikkelen is het noodzakelijk om de psycho-sociale determinanten van roken en stoppen met roken te kennen. Interventies zullen zich immers moeten richten op het veranderen van die determinanten. Daarom zullen in deel I van dit proefschrift drie studies worden gepresenteerd die inzicht geven in de psychosociale determinanten van roken en stoppen met roken.

In hoofdstuk 2 worden rokers en ex-rokers in verschillende fasen van verandering met elkaar vergeleken. Rokers in de vooroverwegersfase, de overwegersfase en de voorbereidingsfase en ex-rokers in de stopfase en de volhoudfase worden met elkaar vergeleken wat betreft de positieve en negatieve uitkomstverwachtingen (verwachte voor- en nadelen van stoppen) en de eigen-effectiviteitsverwachtingen. Ten eerste bleek het patroon van de positieve en negatieve uitkomstverwachtingen door de veranderingsfasen vrijwel identiek aan het patroon dat was gevonden met de zogenaamde "Decisional Balance"-maten (verwachte voor- en naddelen van roken) van het populaire en bij meerdere gedragingen geverifieerde transtheoretische model (TTM). Het "sterke principe van gedragsverandering" en het "zwakke principe wan gedragsverandering" werden namelijk gerepliceerd: Het maximale verschil binnen de eerste vier veranderingsfasen (vooroverwegersfase, overwegersfase, voorbereidingsfase en de stopfase) wat betreft de verwach te voordelen van stoppen was 1,2 standaarddeviatie, terwijl het maximale verschil binnen de eerste vier veranderingsfasen wat betreft de verwachte nadelen van stoppen 0,5 standaarddeviatie was. Deze bevindingen worden beschouwd als een validatie van de operationalisaties van de psychologische constructen (de psycho-sociale determinanten) die in dit proefschrift worden gebruikt. Ten tweede werd de hypothese die gevormd was op grond van het zogenaamde $O$-patroon bevestigd: De verwachte voordelen van stoppen discrimineerden beter tussen de vroege veranderingsfasen (vooroverwegersfase, overwegersfase en voorbereidingsfase) terwijl de eigeneffectiviteitsverwachtingen beter discrimineerden tussen de late veranderingsfasen (stopfase en volhoudfase). Dit differentiële patroon van de verwachte voordelen en de eigen-effectiviteitsverwachtingen in de verande- 
ringsfasen zou consequenties kunnen hebben voor de inhoud van fase-specifieke interventies: Vooroverwegers zouden alleen informatie nodig hebben over de voordelen van stoppen, overwegers zouden informatie nodig hebben over de voordelen van stoppen in combinatie met informatie bedoeld om de eigen-effectiviteit te verhogen terwijl voorbereiders alleen deze eigeneffectiviteitsverhogende informatie nodig zouden hebben.

In hoofdstuk 3 wordt een veranderingsfasenmodel gepresenteerd dat rokers in vier in plaats van drie fasen indeelt. Binnen de groep rokers die niet van plan is om binnen zes maanden een stoppoging te doen worden nu twee subgroepen onderscheiden. Ten eerste worden de rokers onderscheiden die in ieder geval niet van plan zijn om binnen vijf jaar te stoppen met roken of van plan zijn om altijd te blijven roken, de zogenaamde ongemotiveerden. Ten tweede worden de rokers onderscheiden die wel van plan zijn om binnen vijf jaar te stoppen met roken, de zogenaande vooroverwegers. In dit nieuwe veranderingsfasenmodel worden nu dus vier groepen met rokers onderscheiden met toenemende bereidheid om hun (rook)gedrag te veranderen: ongemotiveerden, vooroverwegers, owerwegers en voorbereiders. In dit hoofdstuk wordt het patroon van de verwachte voor- en nadelen van stoppen en van de effectiviteitsverwachtingen door de fasen en de predictieve validiteit van het model onderzocht. Eerst wordt er echter een principale component analyse gedaan met betrekking tot de verwachte voor- en nadelen en de eigeneffectiviteitsverwachtingen. Binnen de verwachte voordelen van stoppen werden vier factoren gevonden: de verwachte lange en korte termijn fysieke uitkomsten, de verwachte sociale uitkomsten en de verwachte zelf-evaluatieve uitkomsten. Binnen de eigen-effectiviteitsverwachtingen werden twee factoren gevonden: eigen-effectiviteit met betrekking tot het niet-roken in sociale situaties en eigen-effectiviteit met betrekking tot het niet-roken in emotionele situaties.

Ten eerste werden de rokers in de vier fasen vergeleken op hun scores op deze factoren. Het bleek dat het aantal factoren dat tussen twee opeenvolgende fasen discrimineerde afnam met de toename van de bereidheid om te stoppen: Ongemotiveerden en vooroverwegers verschilden op alle factoren, vooroverwegers en overwegers verschilden op een aantal factoren terwijl overwegers en voorbereiders slechts op éen factor verschilden, namelijk op de verwachte zelf-evaluatieve uitkomsten. Ten tweede liet deze studie zien dat de veranderingsfasen predictieve validiteit hebben: Vooroverwegers lieten, gemeten met drie gedragsmaten, significant meer stopactiviteit zien dan ongemotiveerden, na drie en na veertien maanden. Overwegers lieten significant meer stopactiviteit zien dan vooroverwegers op twee gedragsmaten na drie maanden en op één gedragsmaat na veertien maanden. Voorbereiders lieten significant meer stopactiviteit zien dan overwegers, op alle drie gedragsmaten na drie maanden en op twee gedragsmaten na veertien maanden. Er werd geconcludeerd dat de vier veranderingsfasen sterke predictieve validiteit hebben maar dat het onderscheid tussen vooroverwegers en overwegers sterker door de psycho-sociale factoren dan door de predictieve validiteit 
werd ondersteunt.

In hoofdstuk 4 wordt de invloed van verwachte uitkornsten op intentie en gedrag verder onderzocht. Het Zelf-Evaluatie-Motivatie model schetst enkele mogelike relaties tussen de verwachte externe uitkomsten (verwachte negatieve uitkomsten, verwachte fysieke uitkomsten, verwachte sociale uitkomsten, verwachte zelf-evaluatieve uitkonsten), twee zelf-evaluatie remmende cognitieve mechanismen en intentie en gedrag.

Met multivariate regressie-analyses werd de eerste hypothese geverifieerd: Zelf-evaluatie medieerde inderdaad de invloed van de andere cognitieve factoren op intentie en gedrag. De tweede hypothese, verwachte externe uitkomsten beïnloeden de zelf-evaluatie alleen als zelf-evaluatie remmende cognitieve mechanismen dat toelaten, werd slechts deels geverifieerd: De verwachte fysieke lange termijn uitkomsten hadden alleen invloed op zelfevaluatie als de attentionele veranderingsprocessen dat toelieten. Verder werd de invlloed wan beide zelf-evaluatie remmende cognitieve mechanismen dissonantie reducerende cognities en attentionele veranderingsprocessen - op zelf-evaluatie deels gemedieerd door de verwachte externe uitkomsten.

Concluderend wordt gesteld dat zelf-evaluatie een centrale psycho-sociale determinant is van intentie en gedrag en dat interventies de negatieve zelfevaluatie dienen te laten toenemen. Een toename in negatieve zelf-evaluatie zou kunnen worden bewerkstelligd door: 1) een toename van de verwachte externe uitkomsten zoals de lange en korte termijn fysieke uitkomsten en de sociale uitkomsten; 2) het herstructureren van dissonantie reducerende cognities; 3) een toename van attentionele veranderingsprocessen; 4) het expliciet wijzen op de discrepantie tussen iemands persoonlijke standaarden en de consequenties van het rookgedrag.

In deel II zijn de resultaten van de studies in deel I gebruikt on de geïndividualiseerde interventies te ontwikkelen. Tevens wordt in deel II de effectiviteit van deze geîndividualiseerde interventies getoetst.

In hoofdstuk 5 worden drie fasen in de ontwikkeling van een geïndividualiseerde interventies gepresenteerd. In de eerste fase worden de interventiedoelen beschreven op grond van de analyse van psycho-sociale determinanten van roken en stoppen met roken. De interventiedoelen beschrijven de gewenste psychologische toestanden of de gedragstoestanden die door de interventie bewerkstelligd dienen te worden. In de tweede fase wordt voor elk interventiedoel een zogenaamde "tailoring matrix" ontwikkeld. Deze matrix brengt relevante individuele kenmerken in kaart waaraan de informatie - bedoeld om de gewenste toestand te bewerkstelligen - dient te worden aangepast. In deze fase worden de informatieboodschappen geschreven en kunnen de twee clusters van werkingsmechanismen - aanpassing van de informatie en feed-back - in de informatieboodschappen worden verwerkt. Verder wordt de zogenaamde "tailoring vragenlijst" ontwikkeld. Deze is bedoeld om iemands toestand wat betreft relevante kenmerken te meten zodat de interventic aan die individuele kenmerken kan worden aangepast. Tot slot worden de tailoring matrix en de tailoring vragenlijst samengebracht tot het 
"functioneel ontwerp" dat een koppeling maakt tussen individuele kenmerken en de informatieboodschappen die rekening houden met die kenmerken. Het functioneel ontwerp is de basis voor het computerprogramma. In de derde fase wordt er voor gezorgd dat de geindividualiseerde interventie, die is opgebouwd uit verschillende boodschappen die weer aangepast kunnen zijn aan verschillende individuele kenmerken, een coherent geheel vornt. Tot slot dient er voor gezorgd te worden dat er een aantrekkelijke vormgeving is en dat de interventie gemakkelijk leest.

In hoofdstuk 6 worden drie geïndividualiseerde interventies getest in een gerandomiseerd veld-experiment $(\mathrm{N}=1540)$ met ongemotiveerden, vooroverwegers, overwegers en voorbereiders. Er wordt een special soort geïndividualiseerde interventie gebruikt; de geïndividualiseerde fase-specifieke interventie. Fase-specifieke interventies zijn geindividualiseerd in die zin dat ze rekening houden met de veranderingsfase waarin een individu zich bevindt. In dit experiment wordt de fase-specifieke informatie ook nog eens aangepast aan verschillende andere individuele kenmerken.

De rokers werden willekeurig verdeeld over drie experimentele en één controleconditie. Respondenten in conditie 1 ontvingen alleen informatie over de voor- en nadelen van roken en stoppen met roken. Respondenten in conditie 2 ontvingen eigen-effectiviteitverhogende informatie: informatie over het gebruik van vaardigheden om te stoppen met roken. Respondenten in conditie 3 ontvingen een combinatie van beide soorten informatie. Respondenten in conditie 4 , de controleconditie, ontvingen geen informatie. Faseverandering was de primaire uitkomsmat en de data werden gestratificeerd naar fase geanalyseerd. Op basis van de studie gepresenteerd in hoofdstuk 2 werd verwacht dat ongemotiveerden het meeste bat zouden hebben bij informatie over de voor- en nadelen, vooroverwegers en overwegers het meeste baat zouden hebben bij de combinatie van beide soorten informatie en dat voorbereiders het meeste baat zouden hebben bij eigen-effectiviteitverhogende informatie.

De resultaten lieten zien dat de hypothesen voor rokers met lage bereidheid om te stoppen, ongemotiveerden en vooroverwegers, slechts gedeeltelijk geverifieerd werden: In vergelijking met de controlegroep hadden ongemotiveerden het meeste baat bij een interventie die informatie over de voor- en nadelen bevatte (al dan niet in combinatie met eigen-effectiviteitwerhogende informatie) en vooroverwegers hadden het meeste baat bij een interventie die eigen-effectiviteitverhogende informatie bevatte (al dan niet in combinatie met informatie over de voor- en nadelen). Een strengere test waarin rokers die in een passende conditie zaten werden vergeleken met rokers die in een niet-passende conditie zaten kon dit resultaat echter niet ondersteunen. De hypothesen voor rokers met een hoge bereidheid on te stoppen, overwegers en voorbereiders, werden wel geveriffeerd. Uit de vergelijking met de controlegroep bleek dat overwegers inderdaad het meeste baat hadden bij beide soorten informatie en dat voorbereiders het meeste baat hadden bij eigen-effectiviteitverhogende informatie. Deze resultaten werden 
ondersteund door de strengere test waarin overwegers en voorbereiders in een passende conditie werden vergeleken met overwegers en voorbereiders in een niet-passende conditie.

In hoofdstuk 7 worden de lange termijn resultaten van de geindividualiseerde interventies bestudeerd. Het cohort rokers uit de studie in hoofdstuk 6 werd veertien maanden gevolgd. De drie geindividualiseerde interventies leidden tot meer stoppogingen dan de controlegroep. Echter, geen van de interventies leidde tot een hoger percentage rokers dat al zeven dagen niet gerookt had, in vergelijking met de controlegroep. Alleen de geïndividualiseerde interventie die beide soorten informatie bevatte leidde tot meer continue abstinentie dan de controlegroep. Geconcludeerd wordt dat 6 pagina's geïndividualiseerde informatie over de voor- en nadelen en over vaardigheden om te stoppen zelfs na veertien maanden nog effectief is.

In hoofdstuk 8 worden vier geindividualiseerde interwenties en één gestandaardiseerde interventie met elkaar vergeleken in een gerandomiseerd veld-experiment $(N=752)$ onder ongemotiveerden en vooroverwegers. Er werd een nieuwe geindividualiseerde interventie ontwikkeld, de zogenaande meermalige geïndividualiseerde interventie. Deze interventie bestaat uit drie getndividualiseerde briewen die met bepaalde tussenpozen aan een individu worden aangeboden. In het huidige experiment waren de tussenpozen ongeveer één maand. Deze meermalige geỉndividualiseerde interventie omvatte grofweg dezelfde informatie als de énmalige geïndividualiseerde interventie uit het vorige experiment die beide soorten informatie bevatte (i.c. informatie over verwachte uitkomsten en eigen-effectiviteitverhogende informatie). Terwijl de eerste van de drie individuele interventies was gebaseerd op de schriftelijke tailoring vragenlijst, waren de tweede en derde gebaseerd op een 10 minuten durende telefonische tailoring vragenlijst. Naast deze interventie werden nog twee andere interventies ontwikkeld: een éenmalige en een meermalige geïndividualliseerde interventie, beiden behorende bij en verwijzende naar een bijgeleverde zelf-hulpgids van 46 pagina's. Tot slot werd een gestandaardiseerde "dubbelganger" ontwikkeld. Deze interventie was wat betreft vormgeving en inhoud sterk gelijkend op de éénmalige geindividualiseerde interventies. De interventie was echter niet geïndividualiseerd.

Rokers werden willekeurig verdeeld over vijf condities waarin ze ontvingen: 1) de meermalige geïndividualiseerde interventie in combinatie met de gestandaardiseerde zelf-hulpgids; 2) de meermalige geindividualiseerde interventie zonder zelf-hulpgids; 3) De éénmalige geöndividualiseerde interventie met een gestandaardiseerde zelf-hulpgids; 4) de énmalige geindividualiseerde interventie zonder zelf-hulpgids of; 5) de gestandaardiseerde dubbelganger. De vervolgmeting was na vier maanden.

De resultaten lieten zien dat de éénmalige geïndividualiseerde interventie zonder zelf-hulp gids het niet beter deed dan zijn gestandaardiseerde dubbelganger. De toevoeging van de gestandaardiseerde zelfhulpgids aan een geindividualiseerde interventie bleek alleen bij zware rokers zin te hebben. Verder bleek dat de meermalige geïndividualiseerde interventie effectiever 
was dan de énmalige geïndividualiseen ci interventie.

In hoofdstuk 9 worden drie zelf-hulpinterventies getest in een gerandomiseerd veld-experiment $(\mathrm{N}=843)$ onder ongemotiveerden en vooroverwegers. Een meermalige geïndividualiseerde interventie, een énmalige geindividualiseerde interventie en de zelf-hulpgids worden met een controlegroep vergeleken die geen interventie kreeg en met elkaar.

De resultaten lieten zien dat onder ongemotiveerden alleen de geindividualiseerde interventies het beter deden dan de controlegroep. Verder bleek dat, bij ongemotiveerden, de meermalige geindividualiseerde interventie het beter deed dan beide anderen terwijl er geen verschil was tussen de éénmalige geindividualiseerde interventie en de zelf-hulpgids. Dit patroon van effectiviteit werd ondersteund door de differentiële cognitieve veranderingen die de interventies teweeg brachten: De meermalige interventie leidde tot een significant grotere toename, vergeleken met de controlegroep, wat betreft de verwachte voordelen van stoppen, de eigen-effectiviteitsverwachtingen en de veranderingsprocessen 'bewustwording' en 'omgevingsevaluatie'. De énmalige geïndividualiseerde interventie was iets minder effectief terwijl de zelfhulpgids niet tot detecteerbare cognitieve veranderingen leidde. De verwachte nadelen van stoppen en het veranderingsproces 'sociale bevrijding' werden niet verschillend beïnvloed door de condities. Bij vooroverwegers bleek, tot onze verassing, dat geen van de interventies het beter deed dan de controlegroep of een van de andere interventies. Dit leek met name veroorzaakt door het hoge percentage voorwaardse faseverandering in de controlegroep.

In hoofdstuk 10 worden de bevindingen van de acht gepresenteerde studies besproken. Ten eerste worden de bevindingen besproken met betrekking tot de fysieke, sociale en zelf-evaluatieve verwachte uitkomsten, met betrekking tot de eigen-effectiviteitsverwachtingen in het omgaan met negatieve emoties en sociale situaties en met betrekking tot de dissonantie reducerende cognities en de attentionele veranderingsprocessen. Ten tweede worden de ontwikkeling en de effectiviteit van de geindividualiseerde interventies besproken. Ten derde komen enkele methodologische aspecten van de studies aan bod. Ten vierde worden mogelijke toepassingen beschreven van de geïndividualiseerde interventies en er wordt een typologie van rokers in de vier veranderingsfasen gepresenteerd. Ten vijfde worden aanbevelingen gedaan voor toekomslig onderzoek naar de cognitieve determinanten, de veranderingsfasen en de geindividualiseerde interventies. 
Acknowledgements

Hein De Vries

Jolanda Roijackers

Martijntje Bakker

Gerjo Kok

Gerard van Breukelen

All smokers

Consulted colleagues

Vic Strecher

Wayne Velicer

Reviewers

Marjanda 
Arie Dijkstra was born on November 3, 1961 in Hoogeveen. After he received his HAVO diploma in 1980 at the Lauwers College in Buitenpost, he trained to be a male nurse and he worked in a general hospital in Leeuwarden for five years. In 1985, he started studying at the Academy for Health Care in Groningen and he received his bachelors degree (cum laude) in nursing in 1988. He started studying Health Sciences at Maastricht University in 1989. During his degree he worked as a male nurse on several psychiatric wards and as a research assistant at the Departments of Mental Health Sciences and Medical Psychology at Maastricht University. In 1993 he received his Masters Degree in Mental Health Sciences and he started research at the Department of Health Education which culminated in this thesis in 1997. During his research he conducted group cognitive/behavioral therapy for smokers at Municipal Services in Limburg. After finishing his thesis Arie worked as a post-doc researcher at the Department of Health Education. As of Februari 11998 he works as an assistant professor at the Department of Psychology at Leiden University. 